\title{
Optical Tweezer Micromanipulation of Filamentous Fungi
}

\author{
Graham D. Wright
}

\section{Ph.D. Thesis}

The University of Edinburgh

2007 
This thesis is dedicated to my parents,

for their faultless support in everything I choose to do. 


\section{Abstract}

Optical tweezers have been little used to date in experimental studies of filamentous fungi. The use of single beam and holographic optical tweezer micromanipulation have been explored for this purpose in the present study.

The single beam system used was a simple, compact, easy-to-use, safe and robust optical tweezer setup mounted on a standard research grade light microscope. It was specifically designed to produce high quality images and to be used with brightfield, phase contrast, differential interference contrast and fluorescence optics. The holographic optical tweezer system used involved the creation of multiple traps and complex patterns of light, and was employed with a range of laser wavelengths.

The various optical tweezer systems were used in a wide range of applications to trap and micromanipulate whole fungal cells, organelles within cells, and synthetic beads. The experimental studies demonstrated how optical tweezers can be used to: unambiguously determine whether hyphae are actively homing towards each other; move the Spitzenkörper and change the pattern of hyphal morphogenesis; create 'pseudowalls' of light to control hyphal growth over extended distances; make piconewton force measurements; investigate the tethering of organelles within cells; mechanically stimulate hyphal tips; produce stable, fixed arrays of cells; and, deliver chemicals to localized regions of hyphae. A significant finding was that germ tubes seem to generate significantly lower growth forces than leading vegetative hyphae. An assessment of the photodamage caused to spores showed that ungerminated spores could be trapped for short periods of time without damage, but could not germinate whilst being continuously trapped for long periods. However once germinated, spores continually trapped for 25 min exhibited no deleterious effects with regard to conidial anastomosis tube (CAT) growth, homing or fusion. 


\section{Acknowledgements}

Many thanks are due to the following people who have helped my PhD evolve either through collaboration or through their advice, support, friendship and inspiration.

My supervisors:

Nick Read

for his constant enthusiasm for my work, guidance that will be with me forever and the legendary annual Eigg retreat

Wilson Poon for his enthusiasm, sound advice and interest in collaborating across the disciplines

The University of Edinburgh:

Jochen Arlt

Laurence Wilson

Andy Garrie

Tom Nowakowski

Marcel Reuter

Nhan Pham

\author{
Andrew Grant \\ Eirini Theofanidou \\ Emmelyn Graham \\ Dave Kelly \\ Richard Parton \\ The Sawin lab
}

All members of the Fungal Cell Biology Group, past and present, especially Gabriela Roca, Pat Hickey, Pete Marris (the undisputed Sigmaplot King), Verena Seidl, Shoujer Kuo, Sam Swift and Mari Valkonen.

\section{External collaborations:}

Chris Hunt - USDA Forest Products Laboratory, WI, USA. Porous silica beads

Dan Burnham \& David McGloin - St. Andrews, UK. Holographic optical tweezers

Daniel Veith \& Reinhard Fischer - University of Karlsruhe, Germany. Aspergillus nidulans

Greg Jedd \& Snezhana Oliferenko - Temasek Life Sciences Laboratory, Singapore.

Schizosaccharomyces pombe

André Fleißner \& Louise Glass - University of California, Berkeley, CA, USA. Neurospora crassa MAK-2-GFP strain

Michael Frietag - Oregon State University, OR, USA. N. crassa H1-GFP strain

My parents and family for all the support, subsidies and constructive nagging

Charlie for being the perfect girlfriend, including her unique brand of encouragement

Thanks to the following societies for their stimulating meetings and generous travel bursaries: BMS, RMS, MSJ, SPIE and BSCB

This work was funded by the Engineering and Physical Sciences Research Council 
I am among those who think that science has great beauty. A scientist in his laboratory is not only a technician: he is also a child placed before natural phenomena which impress him like a fairy tale.

Marie Curie (1867 - 1934) 


\section{Contents}

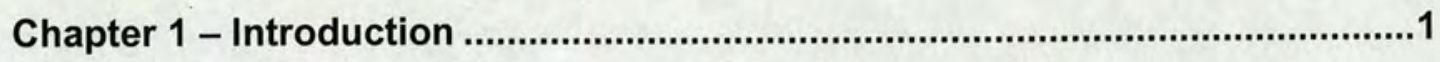

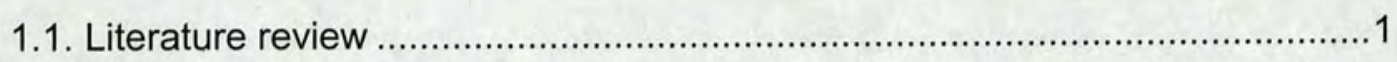

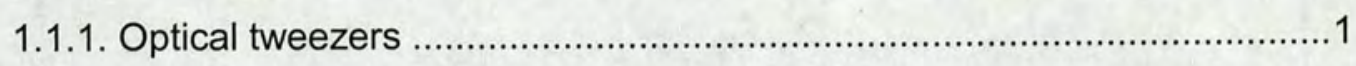

1.1.1.1. Basic physics of optical tweezers .............................................. 1

1.1.1.2. Biological applications of optical tweezers ...................................... 4

1.1.1.3. Photodamage to cells from optical tweezers..................................4

1.1.1.4. Applications of optical tweezers in fungal biology ...........................6

1.1.2. Aspects of fungal biology relevant to this thesis ................................. 8

1.1.2.1. Conidial germination and fusion ............................................... 10

1.1.2.2. Hyphal tip growth and the Spitzenkörper .................................... 11

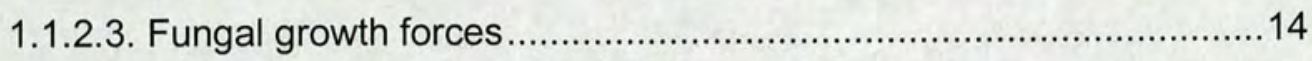

1.1.2.4. Woronin bodies and septal pore plugging .................................. 14

1.2. General introduction to the research in this thesis......................................15

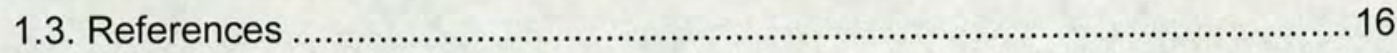

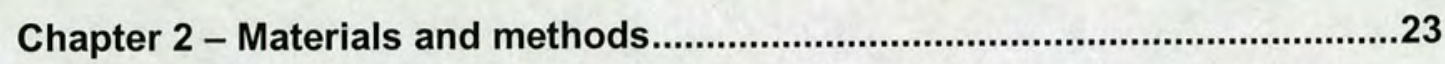

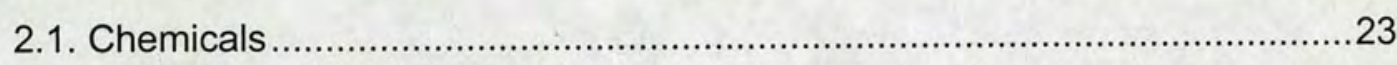

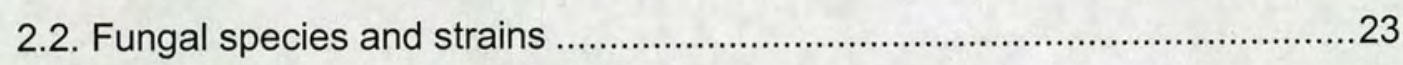

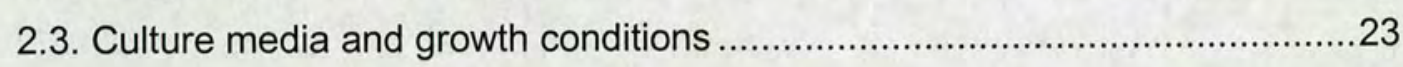

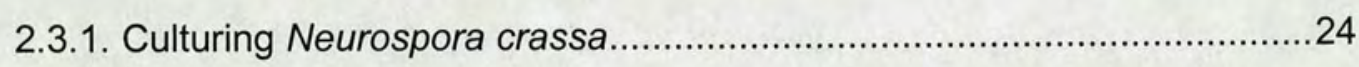

2.3.1.1. Neurospora crassa culture media..............................................24

2.3.1.2. Neurospora crassa storage .......................................................26

2.3.1.2.1. Silica gel for long term storage of conidia .............................26

2.3.1.2.2. Cryopreservation for long term storage ................................26

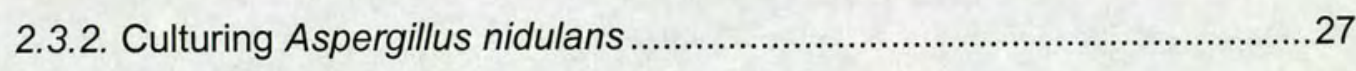

2.3.2.1. Aspergillus nidulans culture media ..............................................2

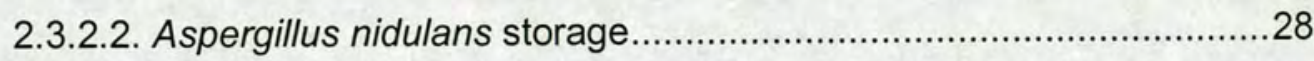

2.3.3. Culturing Schizosaccharomyces pombe............................................29

2.3.3.1. Schizosaccharomyces pombe culture media................................29

2.3.3.2. Schizosaccharomyces pombe storage ........................................ 31 


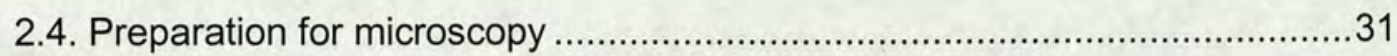

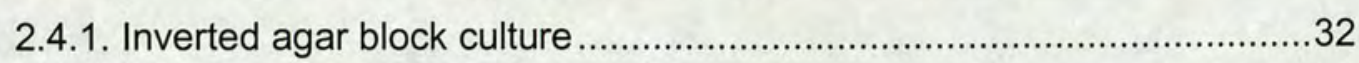

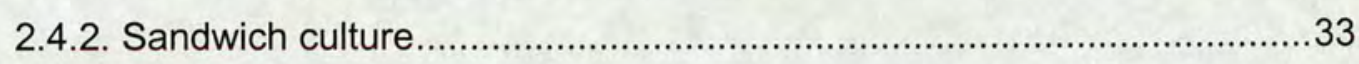

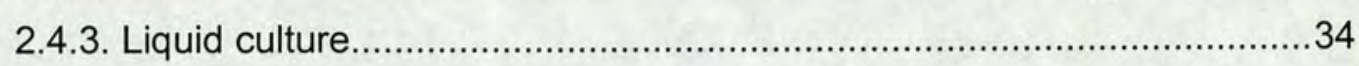

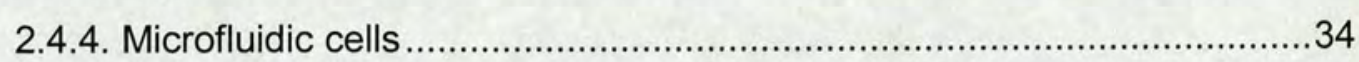

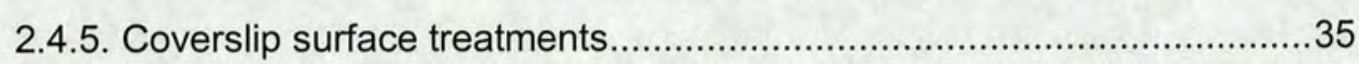

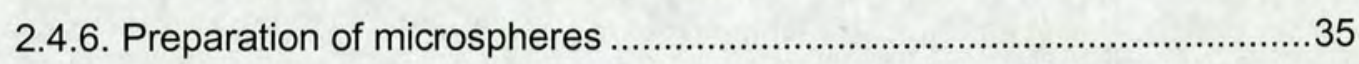

2.4.7. Preparation of dyes for fluorescence microscopy ................................36

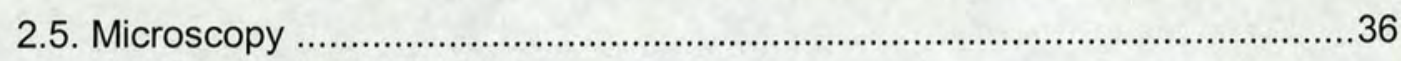

2.5.1. Bright-field, phase contrast and differential interference contrast

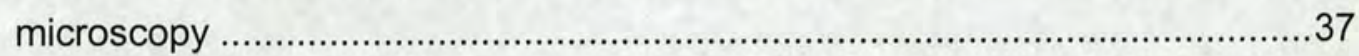

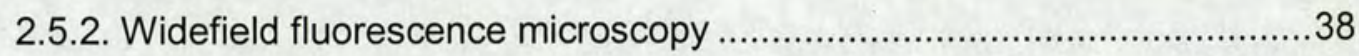

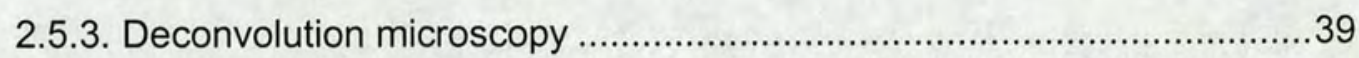

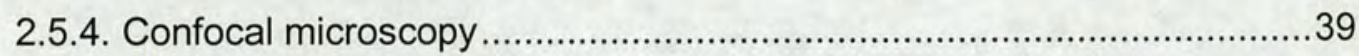

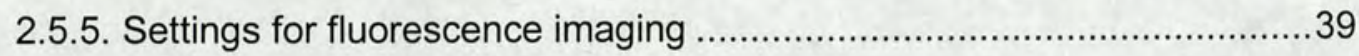

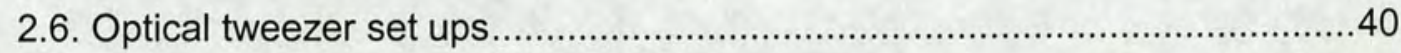

2.6.1. Single-trap optical tweezer systems .................................................40

2.6.2. Dual-trap optical tweezer system .....................................................42

2.6.3. Holographic optical tweezer system ............................................... 42

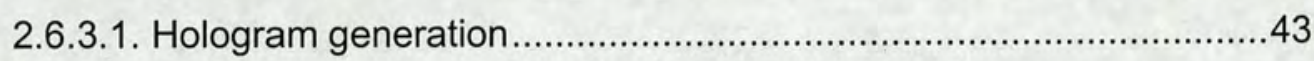

2.6.4. Force calibration for optical tweezers ..............................................44

2.7. Digital image processing and animations ................................................ 44

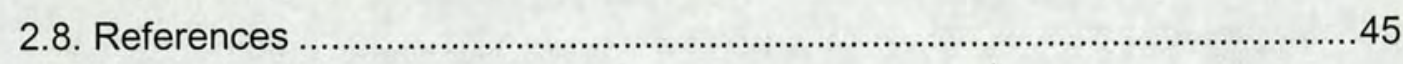

Chapter 3 - Manipulating whole fungal cells with optical tweezers .................47

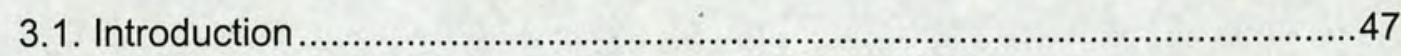

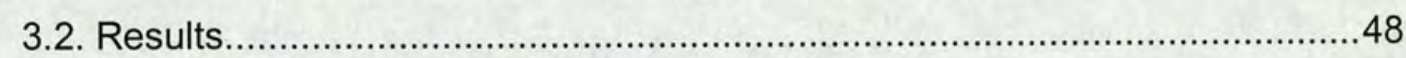

3.2.1. Repositioning and re-orientation of conidia .....................................48

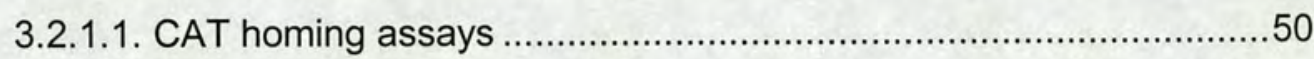

3.2.1.2. MAP Kinase oscillatory signalling during CAT homing ..................51

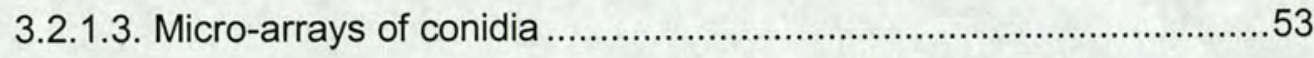

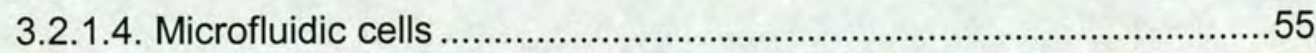

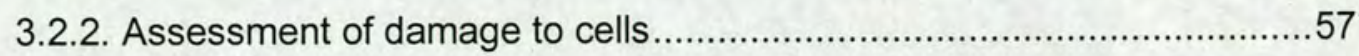

3.2.2.1. Short term trapping of ungerminated conidia ...............................5 57 


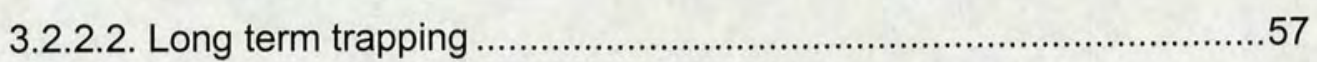

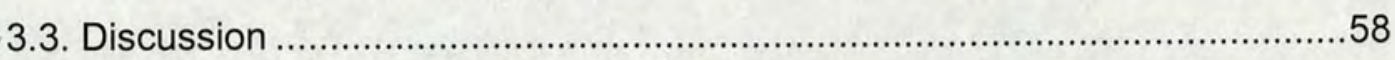

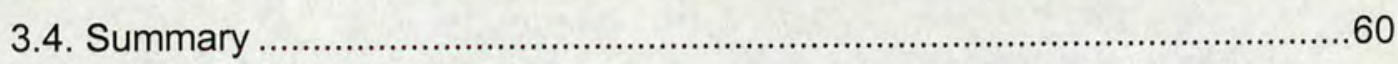

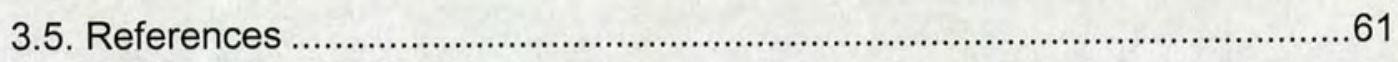

Chapter 4 - Manipulating organelles within fungal cells

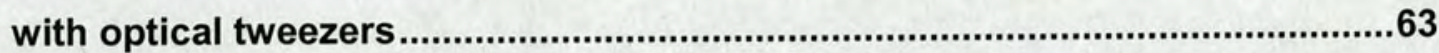

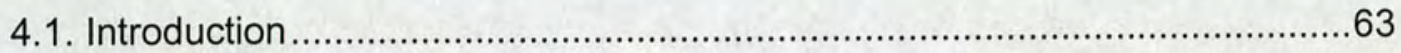

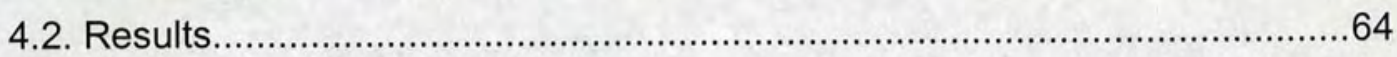

4.2.1. Manipulating organelles of high refractive index...............................64

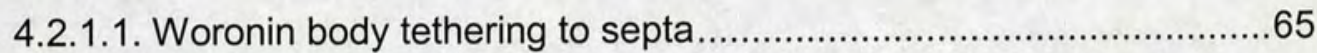

4.2.1.2. Manipulating ectopically produced crystals within cells..................65

4.2.2. Manipulating organelles of low refractive index .................................66

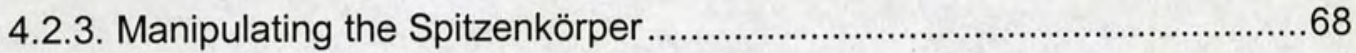

4.2.3.1. Single Gaussian tweezer experiments........................................68

4.2.3.2. Holographic multiple Gaussian tweezer experiments ....................70

4.2.3.3. Holographic continuous light patterns ......................................... 70

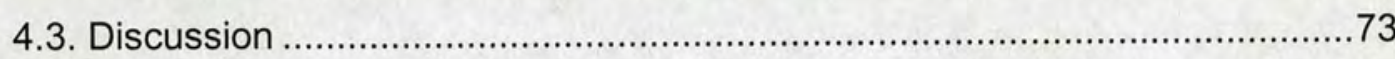

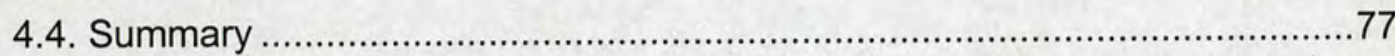

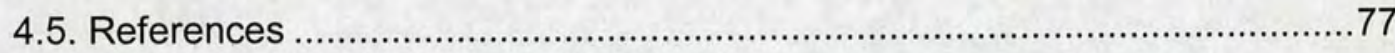

Chapter 5 - Manipulating microspheres with optical tweezers as experimental tools to study fungal cells ........................................................79

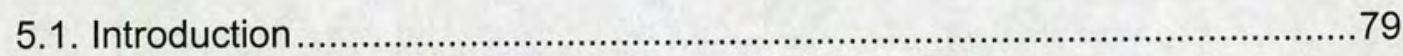

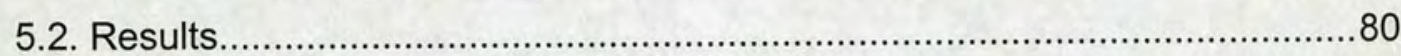

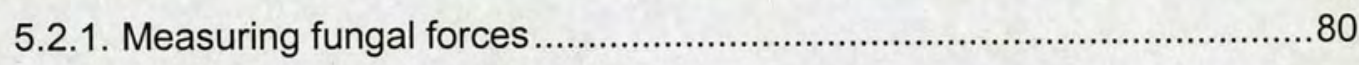

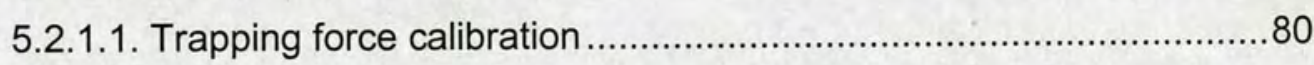

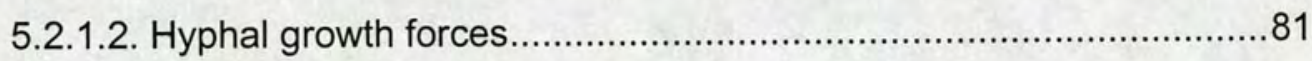

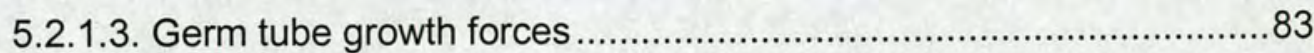

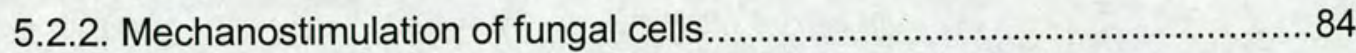

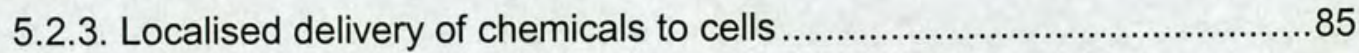

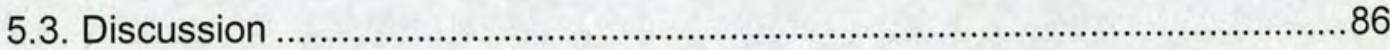

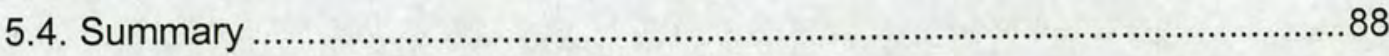


Chapter 6 - Overall summary and future work .................................................91

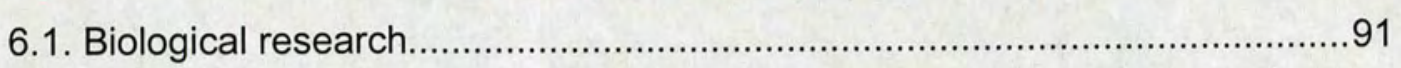

6.2. Future developments to the optical tweezers ...........................................94

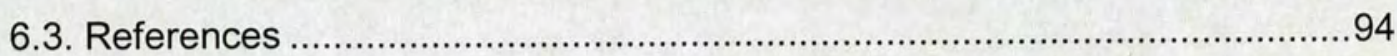

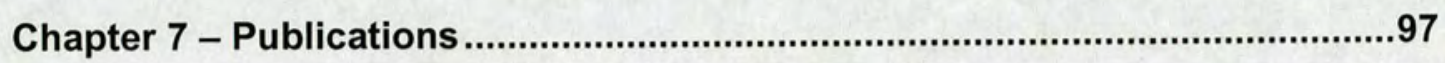

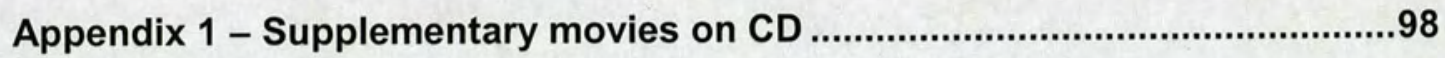




\section{Abbreviations}

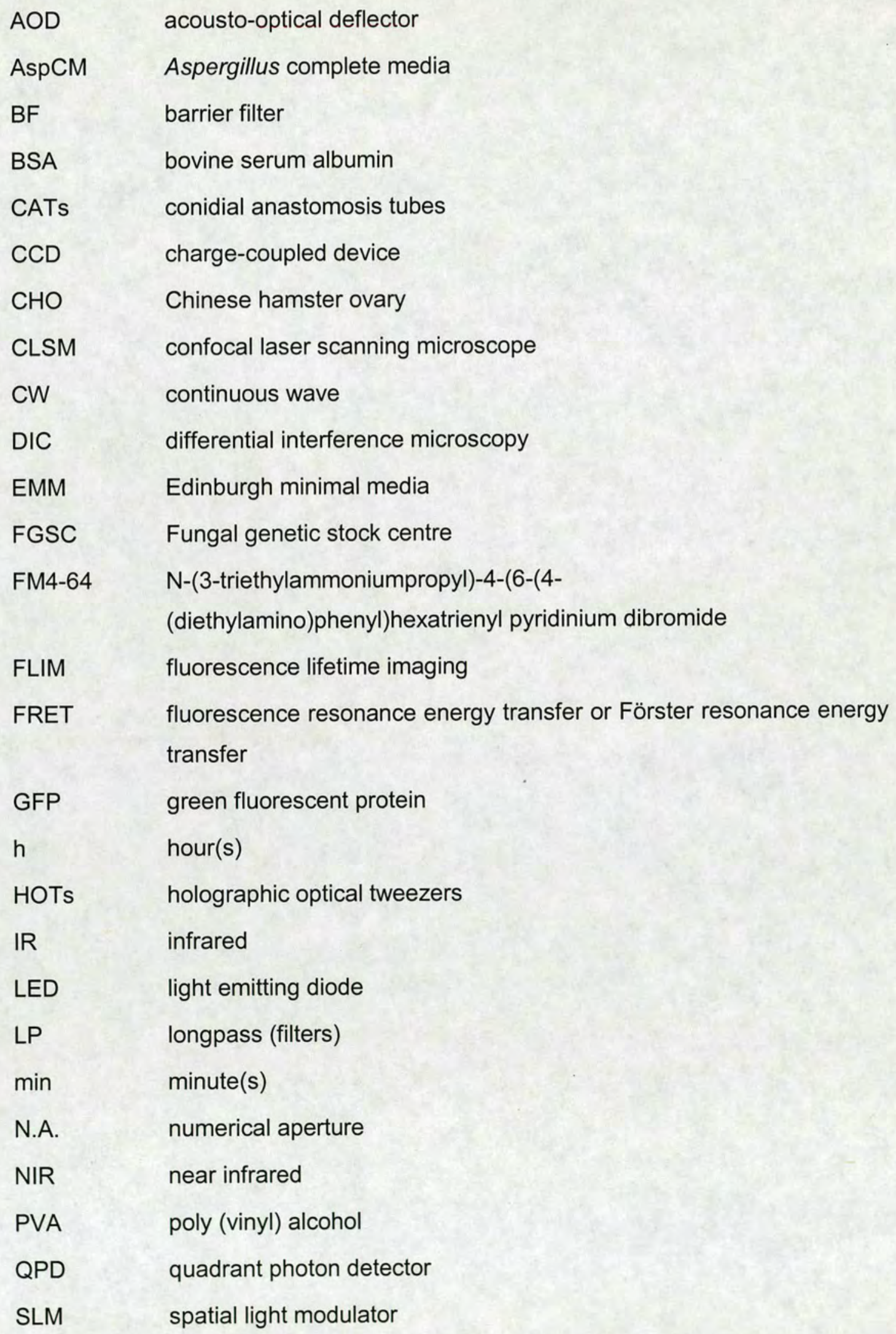


Spk Spitzenkörper

$\mathrm{TEM}_{00} \quad$ laser mode defining a Gaussian intensity profile

$\mathrm{v} / \mathrm{v} \quad$ volume by volume

w/v weight by volume 


\section{Chapter 1 - Introduction}

\subsection{Literature review}

\subsubsection{Optical tweezers}

\subsubsection{Basic physics of optical tweezers}

Optical tweezers, also known as optical traps or laser tweezers, were first described in 1986 by Ashkin and colleagues working at the AT\&T Bell Labs (Ashkin et al., 1986). After many years of work in the area of particle trapping using optical forces, with two counterpropagating laser beams or single-beam optical levitation of particles (Ashkin, 1970; Ashkin and Dziedzic, 1971), the next step was to create a 3D trap from a single laser - an optical tweezer (reviewed in Ashkin, 1997). Optical tweezers permit the non-invasive trapping and micromanipulation of inert and biological objects by means of optical radiation alone. A wealth of work has since amassed, describing both the development of the technique and the application of it across many disciplines. A number of general reviews on optical tweezers are available (Block, 1990; Chu, 1991; Block, 1992; Kuo and Sheetz, 1992; Ashkin, 1997; Sheetz, 1998; Greulich, 1999; Molloy and Padgett, 2002; Neuman and Block, 2004).

In practice optical tweezers, using the forces generated when light interacts with matter, trap objects near the point of focus of an objective lens. To understand the origin of trapping forces, one needs to appreciate that when photons are absorbed, reflected or refracted by an object, the momentum they possess is changed, i.e. the object exerts a force on the photons (an action force). Newton's third law states that for every action force there is a corresponding reaction force that is equal in magnitude and opposite in direction. The object exerting the force on the photons will therefore experience this reaction force (Fig. 1.1). Optical tweezers require an intense laser light source, tightly focused by an objective lens of high numerical aperture (N.A.) $)^{1}$. They produce forces in the piconewton $(\mathrm{pN})$ range, from laser powers in the milliwatt $(\mathrm{mW})$ range, which are sufficient to trap microscopic particles and move them relative to their surroundings. For a particle to be trapped efficiently it must have a refractive index that is sufficiently higher than its surroundings.

\footnotetext{
${ }^{1}$ Numerical aperture: a parameter expressing the ability of an objective lens to gather light and the resolving power. A higher N.A. gives greater resolution. A high value is required for optical tweezers to achieve stronger trapping.
} 
Ashkin defined two forces to explain the effect of light on a material, the gradient force (Fig. 1.2, $\mathrm{F}_{\text {grad }}$ acting transversely to the direction of beam propagation) and scattering force (Fig 1.2, $\mathrm{F}_{\text {scat }}$ acting in the direction of the beam). In a Gaussian beam profile ${ }^{2}$ the refraction of the light rays $\mathrm{a}$ and $\mathrm{b}$ creates forces $\mathrm{F}_{\mathrm{a}}$ and $\mathrm{F}_{\mathrm{b}}$ (Fig. 1.2). Ray $\mathrm{b}$ is of higher intensity, so that $F_{b}>F_{a}$, causing the object to move towards the region of higher intensity. When the object is at the centre of the beam, $F_{a}=F_{b}$ and there will be no gradient force component (Fig. 1.2A)(Ashkin, 1997).

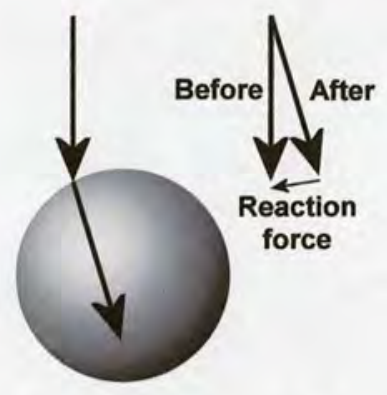

Figure 1.1 A ray diagram depicting the interaction of a single ray of light with a transparent sphere (e.g. a polystyrene bead). The sphere refracts the light, changing its direction and therefore momentum (an action force). The resultant reaction force is opposite in direction and equal in magnitude (Newton's third law).

In Fig. 1.2A, $\mathrm{F}_{\text {scat }}$ would also move the sphere in the direction of the beam propagation. To overcome this and create a truly $3 \mathrm{D}$ trap the laser is tightly focused through a microscope objective lens (Fig. 1.2B). This creates a larger gradient force back towards the laser source, overcoming $\mathrm{F}_{\text {scat, }}$, and results in an overall net force trapping the object in 3-dimensions near the point of focus. The objective lens must be of a high N.A. to create a steep gradient of light and improve trapping in 3-dimensions. Two distinct ways of explaining and understanding the forces generated by optical tweezers exist depending upon the size of the objects beings trapped; the Mie regime and the Rayleigh regime. The Mie regime, is valid when the size of the object being trapped exceeds the wavelength of the light. Here, the schematic explanation we have provided using ray optics (Fig. 1.2) is a reasonable one. In the Rayleigh regime, however, the object is smaller then the wavelength of light, the forces are calculated in terms of the electrical field near the trapped object in a wave picture (Molloy et al., 2002).

\footnotetext{
${ }^{2}$ Gaussian beam: named after Johann Karl Friedrich Gauss (1777-1855). A beam whose intensity profile matches that of a Gaussian, or 'normal' distribution, a symmetrical curve peaking in the centre (Fig. 1.2). This is the intensity profile of the so-called TEM ${ }_{00}$ mode of a laser.
} 

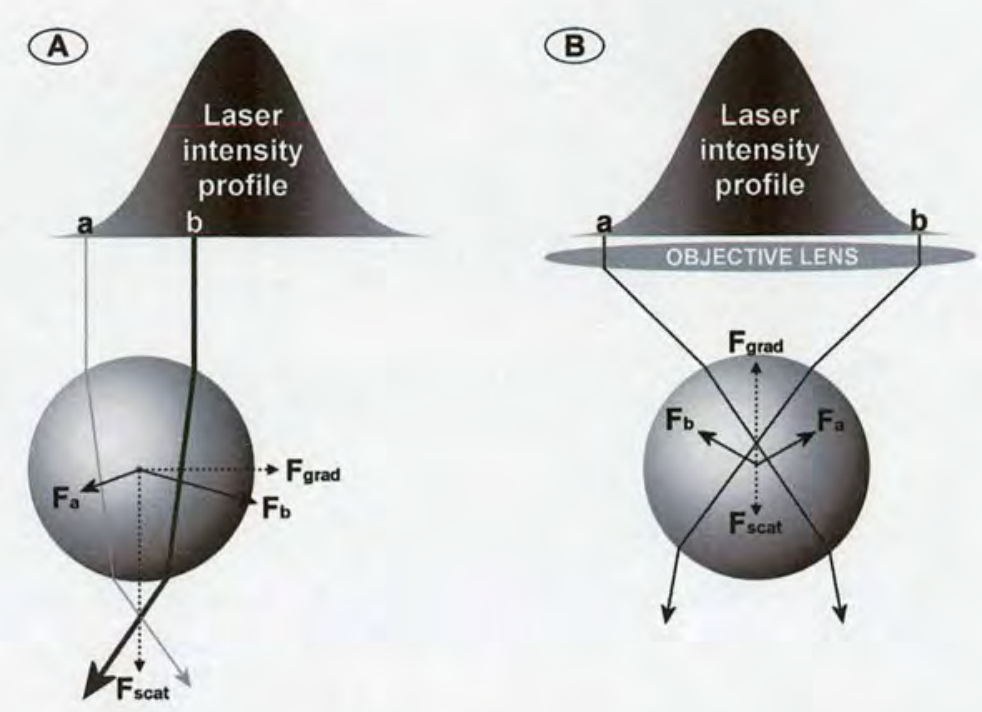

Figure 1.2 Ray diagrams showing the interaction of Gaussian laser light with a transparent sphere. $a$ and $b$ represent rays of light, $F_{a}$ and $F_{b}$ correspond to the forces resulting from the interaction of the rays with the sphere; the length of the arrow represents the relative size of the force. $F_{\text {grad }}=$ gradient force component, $F_{\text {scat }}=$ scattering force component. $(A)$ As the centre of the laser beam is more intense, the ray from this region generates a greater force than the ray from the periphery of the beam. Therefore the net reaction force pushes the sphere to the right as you look at it. Upon reaching the centre of the beam there would no longer be a net gradient force, the sphere would be trapped in $x$ and $y$ directions, but the scattering force would remain, pushing the sphere away from the laser source in the $z$ direction. (B) To also trap in the $z$ direction the beam is focused by an objective lens, creating a backward force towards the point of focus and resulting in the sphere being trapped in 3D. In both cases the sphere is attracted to and trapped in the region of highest light intensity, which in (B) is at the point of focus.

Once trapped the object can be moved either by moving the microscope stage or by moving the trap position within the field of view (e.g. with galvanometric mirrors). The sizes of particles that can be trapped range from large objects such as whole eukaryotic cells down to particles of a few tens of nanometers in size. Often, beads are used as 'handles' - they are attached to biological objects of interest (from DNA molecules to cells) and then the beads are trapped and manipulated. The trapping force produced can be adjusted by changing the laser power, the two being linearly related. More recently, multiple traps systems have been implemented (Grier and Roichman, 2006). These multiple traps can be generated by a number of different approaches; 1) by rapidly scanning a laser beam and time sharing its focus between numerous sites using galvanometric mirrors, piezo actuated mirrors (Mio et al., 2000) or acousto-optical deflectors (Visscher et al., 1996), 2) by splitting a single beam into multiple trap sites, using diffractive optical elements (Dufresne and Grier, 1998) such as spatial light modulators (SLMs), termed holographic optical tweezers (HOTs) (Liesener et 
al., 2000), or by the generalized phase contrast technique (Eriksen et al., 2002a; Eriksen et al., 2002b).

\subsubsection{Biological applications of optical tweezers}

Optical tweezer micromanipulation of living cells provides a powerful method for investigating many aspects of cell biology. Optical tweezers can be used to manipulate whole living cells or organelles directly, deliver molecules in microspheres to localized cell regions, or mechanically stimulate cells by pushing trapped objects against them. Using light to manipulate cells has the following advantages over the more traditional mechanical methods of micromanipulation (such as micropipettes): it is much less invasive and can inflict negligible damage and stress to cells (see below); it allows objects within living cells to be trapped without puncturing the cell wall or plasma membrane; it offers very precise computer control of the position of the object being manipulated; and it allows instant trapping or release of objects (Wright et al., 1990; Block, 1990; Berns et al., 1991; Kuo et al., 1992; Weber and Greulich, 1992; Svoboda and Block, 1994; Ponelies et al., 1994; Sheetz, 1998; Molloy et al., 2002). Under favourable circumstances, the trapping forces can be measured (and controlled) very precisely. Optical tweezers have been used in a wide range of biological applications (Table 1).

\subsubsection{Photodamage to cells from optical tweezers}

One concern frequently raised by biologists is the potential damage caused to cells by the intense laser irradiation used for optical trapping. Commonly in other techniques, such as fluorescence microscopy, the aim is to reduce the dose of light received by cells to a minimum to limit any phototoxic effects. However, judicious selection of the optimal combination of laser wavelength, laser power and duration of exposure for the biological sample of interest can limit any potential damage. Optical tweezers are usually constructed based on a near infrared (NIR; 700-1500 nm) laser. Absorption of light of these wavelengths by biological samples is limited to some chromophores, whilst the main constituents of cells (water, proteins and nucleic acids) exhibit only very weak absorption (Mohanty et al., 2006). A number of papers have addressed the issues of photodamage across a range of species and cell types (König et al., 1995; Liang et al., 1996; Neuman et al., 1999; Leitz et al., 2002; Sacconi et al., 2005; Ayano et al., 2006; Mohanty et al., 2006). 
Table 1.1 Some biological applications in which optical tweezers have been employed. A resource letter written by Lang and Block (2003) provides a list of nearly 400 papers on the main topics of within the field of optical trapping.

\begin{tabular}{|c|c|}
\hline Applications & References \\
\hline Manipulating cells to redirect their growth & $\begin{array}{l}\text { Leitz et al., 1995; Bracker et al., 1997; } \\
\text { Erlicher et al., 2002; Stevenson et al., } \\
2006\end{array}$ \\
\hline Manipulating organelles within cells & $\begin{array}{l}\text { Ashkin and Dziedzic, 1989; Berns et al., } \\
\text { 1992; Ponelies et al., 1994; Leitz et al., } \\
\text { 1995; Tolic-Norrelykke et al., } 2005\end{array}$ \\
\hline $\begin{array}{l}\text { Isolating individual cells, organelles and } \\
\text { chromosomes }\end{array}$ & $\begin{array}{l}\text { Leitz et al., 2003; Liu et al., 2004; Fuller et } \\
\text { al., } 2006\end{array}$ \\
\hline $\begin{array}{l}\text { Measuring the forces produced by motor } \\
\text { proteins and RNA polymerases }\end{array}$ & $\begin{array}{l}\text { Block et al., 1989; Ashkin et al., 1990; } \\
\text { Block et al., 1990; Kuo and Sheetz, 1993; } \\
\text { Finer et al., 1994; Wang et al., 1998; } \\
\text { Block et al., 2003; Greulich, } 2005\end{array}$ \\
\hline $\begin{array}{l}\text { Measuring the biophysical properties of } \\
\text { DNA }\end{array}$ & $\begin{array}{l}\text { Cluzel et al., 1996; Wang et al., 1997; } \\
\text { Greulich and Pilarczyk, 1998; Baumann } \\
\text { et al., 2000; Greulich et al., 2000; Lang et } \\
\text { al., } 2003\end{array}$ \\
\hline Fusing cells & Block, 1992 \\
\hline Providing localized stimulation to cells & $\begin{array}{l}\text { Negulescu et al., 1996; Wei et al., 1999; } \\
\text { Wang et al., } 2005\end{array}$ \\
\hline Automated cell sorting & Buican et al., 1987; Kuo et al., 1992 \\
\hline
\end{tabular}

Liang et al. (1996) highlighted two wavelengths ranges, 800-850 $\mathrm{nm}$ and $950-990 \mathrm{~nm}$, which were the least harmful to the cloning efficiency of Chinese Hamster Ovary (CHO) cells. The study reported that $740-760 \mathrm{~nm}$ and $\sim 900 \mathrm{~nm}$ were the most harmful. The duration of exposure, irrespective of wavelength, was shown to be a significant factor in a cell's ability to survive (Liang et al., 1996). The results from other studies are consistent with these findings. The damage caused by two wavelengths, 760 and $800 \mathrm{~nm}$, when trapping human spermatozoa and CHO cells were compared by König et al. (1995). Spermatozoa trapped with $70 \mathrm{~mW}$ (at the sample) of $760 \mathrm{~nm}$ light were killed within $35 \pm 20 \mathrm{~s}$. However cells remained motile and alive throughout the $10 \mathrm{~min}$ period of trapping with $800 \mathrm{~nm}$ light. Likewise $\mathrm{CHO}$ cells were unable to form clones after $\geq 60 \mathrm{~s}$ exposure to $760 \mathrm{~nm}$ light, but $50 \%$ cloning efficiency was possible even after $600 \mathrm{~s}$ of exposure to $800 \mathrm{~nm}$ light. Photodamage, measured by the rotation rates of flagella, to bacterial cells of Escherichia coli 
was found to be minimal at $830 \mathrm{~nm}$ and $970 \mathrm{~nm}$ and maximal at $870 \mathrm{~nm}$ and $930 \mathrm{~nm}$ (Neuman et al., 1999). Ayano et al. (2006) reported that cell growth and division of E. coli cells was halted during long term trapping with $1064 \mathrm{~nm}$ light, even with very low laser powers ( $3 \mathrm{~mW}$ at the sample). Cells of the fission yeast Schizosaccharomyces pombe were found to exhibit visible photodamage at $880 \mathrm{~nm}$ but not $830 \mathrm{~nm}$. Despite this, trapping lipid granules in these yeast cells with $830 \mathrm{~nm}$ light at high laser power (several hundred $\mathrm{mW}$ 's) delayed cell division (Sacconi et al., 2005). Mitotic spindle elongation, a temperaturesensitive process, was used to demonstrate that the yeast cells were not substantially heated by the $830 \mathrm{~nm}$ trapping laser. Expression of a stress response gene in the nematode worm Caenorhabditis elegans was measured as an indicator of stress experienced by irradiated cells in a study by Leitz et al. (2002). They found that the most stress was caused at $760 \mathrm{~nm}$ and the least at $810 \mathrm{~nm}$. Consistent with other work, they identified that damage was least severe with lower laser powers and shorter durations of irradiation.

Whilst the exact causes of the photodamage resulting from optical trap irradiation have not been elucidated, they are likely to result from a combination of the photochemical generation of reactive oxygen species, two-photon absorption (even from a continuous wave (CW) laser), or transient local heating (photothermal effects) depending on the sample (König et al., 1995; Neuman et al., 1999; Sacconi et al., 2005; Mohanty et al., 2006). Such phenomena are likely to occur due to the high intensities of light involved. The focusing of a laser (at a power of 10's of milliwatts) to what is essentially a diffraction limited spot creates intensity values of megawatts per $\mathrm{cm}^{3}$ (MWcm${ }^{-3}$; König et al., 1995; Peterman et al., 2003).

A number of studies have addressed the issue of heating resulting from trapping. Peterman et al. (2003) showed experimentally and by mathematical modelling that using a $100 \mathrm{~mW} 1064$ $\mathrm{nm}$ trap on microspheres causes a temperature increase of $0.8^{\circ} \mathrm{C}$ at the focus point. A higher value of $1.15 \pm 0.25^{\circ} \mathrm{C} / 100 \mathrm{~mW}$ was reported in $\mathrm{CHO}$ cells by Liu et al.(1995), but Peterman et al. (2003) claim to have developed a more accurate method to measure this by analyzing the changes in the thermal motion of trapped particles. The values recorded would be higher if it was not for the rapid dissipation of heat from such small volumes.

\subsubsection{Applications of optical tweezers in fungal biology}

Despite the wide variety of biological research making use of optical tweezers, the fungi, particularly the filamentous fungi, have been largely overlooked as subjects for experimentation using this technique. Whole cells and organelles of budding yeast 
(Saccharomyces cerevisiae) have been micromanipulated in a range of studies to evaluate different optical tweezer systems due to their convenient size and shape (Ashkin, 1991; Grimbergen et al., 1993; Daria et al., 2004; Goksor et al., 2004; Sacconi et al., 2005; Lafong et al., 2006). Optical tweezers have also been used more experimentally with yeast cells to: investigate the viscoelastic properties of the cytoplasm (Tolic-Norrelykke et al., 2004); demonstrate the specification of the division plane by the nucleus in the fission yeast (Schizosaccharomyces pombe) (Tolic-Norrelykke et al., 2005), and examine the inhibition of growth of Hanseniaspora uvarum cells by confining them with optically trapped cells of Sacch. cerevisiae (Arneborg et al., 2005).

Berns et al. (1992) were the first to use optical tweezers with filamentous fungi, and showed that organelles could be trapped and moved within hyphae without obvious damage. They found that the order of responsiveness of organelles to trapping was: lipid bodies $>$ nucleoli $>$ mitochondria > chromosomes. Bracker et al. (1997) later showed how optical tweezers could be used to move the Spitzenkörper (see section 1.1.2.2) within a hyphal tip, change the direction of hyphal growth, redistribute secretory vesicles in the cytoplasm, and induce branch formation. Their study provided compelling evidence that the Spitzenkörper is responsible for hyphal growth directionality (also see Fig. 3 in Bartnicki-Garcia, 2002). Of particular interest was their observation that rather than being trapped, the Spitzenkörper was repelled by the laser. The Spitzenkörper is a predominately phase dark structure when observed by phase contrast light microscopy (Lopez-Franco and Bracker, 1996) indicating that, overall, it has a higher refractive index than the surrounding cytoplasm. It had therefore been expected that the Spitzenkörper should be trapped rather than repelled by optical tweezers (Bracker et al., 1997).

Roca et al. (2005a) used optical tweezers in a novel assay to assess unambiguously whether conidial anastomosis tubes (CATs; see section 1.1.2.1) of Neurospora crassa home towards each other. This technique allows a conidium or conidial germlings to be optically trapped and to have its position changed relative to another. Using this method, Roca et al. (2005a) showed that CAT tips were both the sites of chemoattractant secretion and reception. Furthermore, by using mutants defective in signalling, the CAT assay was used to show that (1) cAMP is not the CAT chemoattractant and (2) the mutant soft forms CATs but they are unable to home towards or fuse with other CATs (Fleißner et al., 2005). 
Other applications of using optical tweezers to manipulate filamentous fungi are described in Chapters 3-5 in this thesis. Much of this work has been published (Wright et al., 2005; Burnham et al., 2007; Wright et al., 2007a, b).

\subsubsection{Aspects of fungal biology relevant to this thesis}

Filamentous fungi grow in a very unique manner producing a complex interconnected network of multinucleate, tubular cellular elements called hyphae (Fig. 1.3). These hyphae extend by tip growth and penetrate with force, and by digestion, through the microenvironments which they inhabit. Neurospora crassa, the species which is the main focus of this work, is commonly used as a model filamentous fungus for experimental studies, has been the subject of scientific research for over 80 years since being identified as a contaminant in French bakeries 150 years ago (Perkins and Davis, 2000; Davis, 2000), and was the first filamentous fungus to have its complete genome sequenced (Galagan et al., 2003; Borkovich et al., 2004). The species has both sexual and asexual parts to its life cycle (Fig. 1.4). On solid media, $N$. crassa produces copious quantities of orange multinucleate macroconidia (also referred to as spores throughout this thesis) as a result of the budding and segmentation of aerial hyphae. $N$. crassa can also produce two further types of asexual spores, microconidia, from specialized microconidiophores, and arthroconidia. Aspergillus nidulans is another filamentous fungus used, to a limited extent, in the research described in this thesis. Both $N$. crassa and A. nidulans are relatively closely related ascomycete species and share a number of advantages for use in optical tweezers studies, which include: (1) they are easily cultured in the laboratory and can be analyzed as a monolayer of germinating spores or growing hyphae, (2) they can respond quickly to stimuli, and (3) they are very amenable to molecular biology studies (e.g. tagging proteins with the green fluorescent protein (GFP) (Freitag et al., 2004). N. crassa in particular has further advantages: (1) the hyphae grow rapidly, at rates of up to a maximum of $2.1 \mu \mathrm{m}$ per second (Bracker et al., 1997), (2) it produces large hyphae (up to $20 \mu \mathrm{m}$ in diameter), which are well suited for livecell imaging (Hickey et al., 2002; Freitag et al., 2004; Hickey et al., 2005), and (3) a vast collection of strains and mutants are available (www.fgsc.net). 


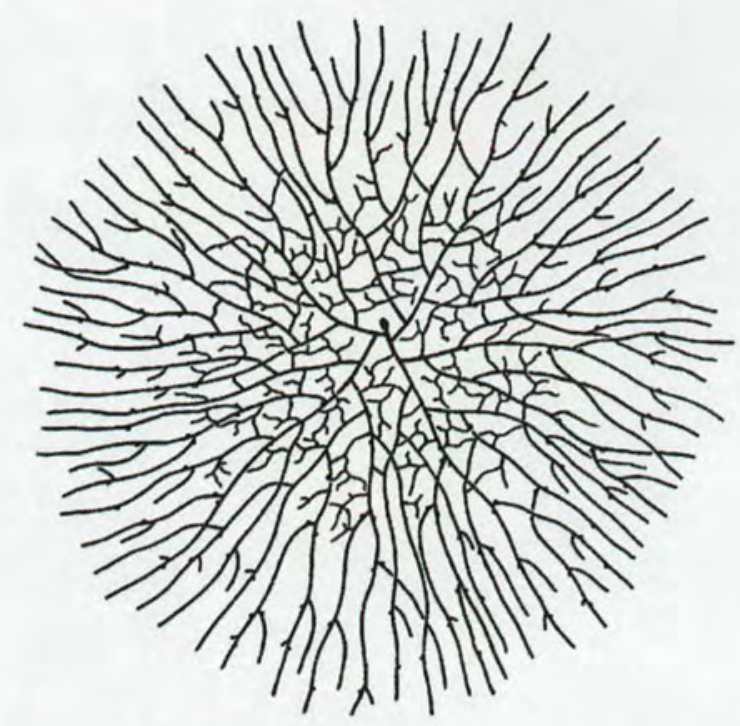

Figure 1.3 Diagram showing the typical appearance of a filamentous fungal colony (Coprinus sterquilinus in this example) grown from a single spore. Leading hyphae at the colony periphery avoid one another, whilst hyphae in the interior of the colony actively fuse. A plate inoculated in the centre with an agar plug produces the same colony morphology (adapted from Buller, 1933).

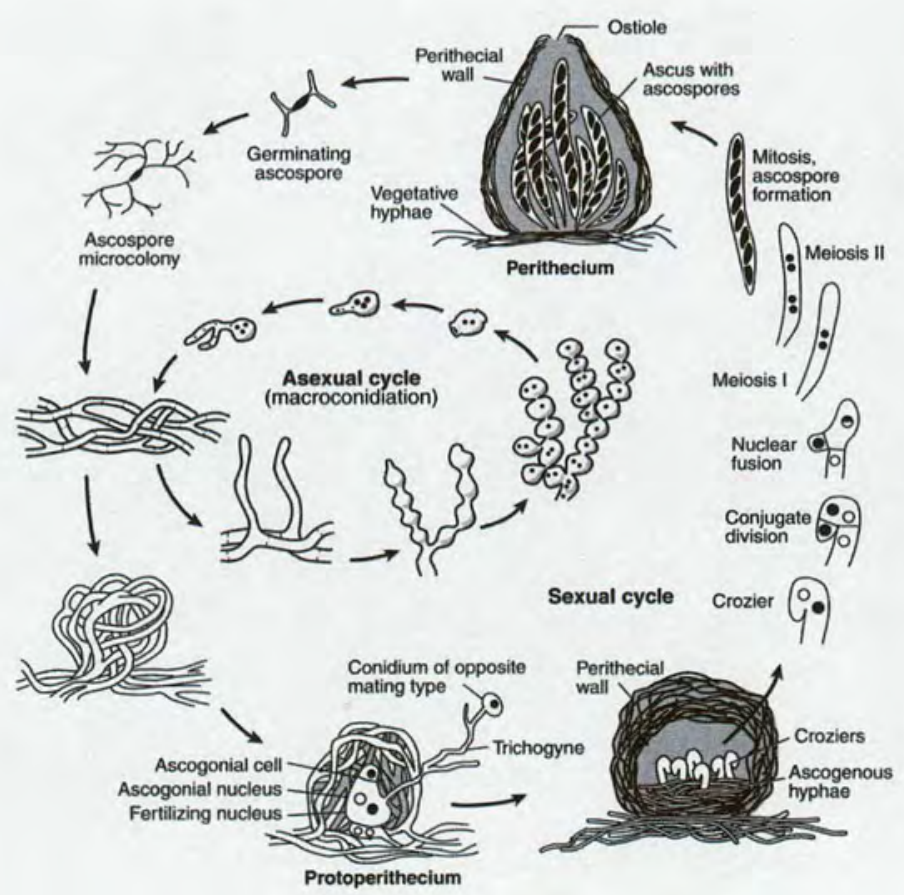

Figure 1.4 The life cycle of Neurospora crassa. The asexual cycle leading to the production of macroconidia is illustrated in the inner sequence. The outer sequence illustrates the main events in the sexual cycle, in which a protoperithecium is fertilized by the fusion of the trichogyne with a conidium of the opposite mating type ( $N$. crassa has two mating types mata and mat-A) and leads to the production of ascospores inside the asci of a perithecium (taken from Davis, 2000). 


\subsubsection{Conidial germination and fusion}

Primary functions of conidia are in asexual reproduction, dispersal and in acting as male fertilising agents during sexual reproduction (Fig. 1.4). Macroconidia, once in a favourable environment, hydrate then initiate a cascade of mRNA expression, biochemical and metabolic changes (Tables 1.2, 1.3; Fig. 1.5)(Schmit and Brody, 1976; d'Enfert, 1997; Kasuga et al., 2005). Conidia undergo isotropic growth following hydration prior to the emergence of a germ tube (d'Enfert, 1997; Araujo-Palomares et al., 2007). Initially most of the organelles (e.g. nuclei and mitochondria) display a more-or-less uniform distribution within growing germ tubes. However, after many hours when cells reach $\sim 150 \mu \mathrm{m}$ in length, the organelle organisation becomes polarised, and a small exclusion zone appears at the tip, followed by the development of a phase dark Spitzenkörper, which is characteristic of mature growing vegetative hyphae (Araujo-Palomares et al., 2007). As the mature colony develops it forms a complex interconnected hyphal network, the mycelium (Fig. 1.3). The colony extends through its environment as a result of tip growth of hyphae at the colony periphery, whilst hyphal fusion occurs a few hundred micrometers behind this region.

During the early stages of colony establishment another type of recently identified specialized hypha called a conidial anastomosis tube (CAT) forms, and this functions in fusing with other CATs to form an interconnected network of germlings (Roca et al., 2005a; Roca et al., 2005b). CATs have been shown to be morphologically and physiologically distinct from germ tubes and under separate genetic control in $N$. crassa (Roca et al., 2005a). Typically they are short, thin and unbranched and can grow from conidia or develop from germ tubes. Although the chemoattractant involved remains elusive there is an increasing understanding of the signalling pathways involved in CAT induction, homing and fusion (Roca et al., 2005a; Read and Roca, 2006). 
Table 1.2 Cytological events associated with the early stages of colony establishment in N. crassa (from Roca et al., 2005a and; Araujo-Palomares et al., 2007).

\begin{tabular}{c}
\hline Hydration \\
\hline $\begin{array}{c}\text { Is } \mathbf{h} \\
\text { Isotropic conidial growth }\end{array}$ \\
\hline Germ tube emergence \\
CAT induction \\
\hline$>\mathbf{3} \mathbf{h}$ \\
\hline CATs start to fuse \\
\hline$>\mathbf{5} \mathrm{h}$ \\
\hline FM4-64 staining can be detected in vesicles in the tip \\
Uniform distribution of organelles \\
\hline 10 $\mathrm{h}$ \\
\hline Exclusion zone establishes at tip \\
'immature Spitzenkörper' develops \\
Increasingly polarized distribution of organelles \\
(mitochondria and nuclei) \\
Phase dark mature Spitzenkörper observed
\end{tabular}

\subsubsection{Hyphal tip growth and the Spitzenkörper}

Vegetative hyphae grow by the phenomenon of tip growth (Bartnicki-Garcia, 2002; Harris et al., 2005; Virag and Harris, 2006). This characteristic growth pattern is also observed in some plant cells (e.g. pollen tubes, root hairs and algal rhizoids) (Heath, 1990) but it is the filamentous fungi that have exploited tip growth as a key feature of their lifestyle (Read, 2007).

Tip growth is achieved by fusion of secretory vesicles with the plasma membrane of the hyphal tip thereby delivering new membrane, new cell wall material and enzymes for wall synthesizing and plasticizing (Bartnicki-Garcia et al., 1995). In 1924 Brunswick coined the term Spitzenkörper (which translates as 'apical body') to describe the structure within hyphal tips which correlates with the multicomponent complex dominated by secretory vesicles, and which is intimately associated with tip growth (Mcclure et al., 1968; Bartnicki-Garcia, 2002; Harris et al., 2005; Virag et al., 2006). The Spitzenkörper can be visualised as a phase dark body with phase contrast microscopy in the tips of growing hyphae of the higher fungi (Girbardt, 1957; Grove and Bracker, 1970; Lopez-Franco et al., 1996; Bartnicki-Garcia, 
2002). Ultrastructural analyses of the Spitzenkörper have shown that it is typically composed of a core rich in actin, usually surrounded by microvesicles (40-50 $\mathrm{nm}$ in diameter), larger apical vesicles (70-90 $\mathrm{nm}$ diameter), microtubules, actin microfilaments and ribosomes (Grove et al., 1970; Howard, 1981; Roberson and Fuller, 1988). Vesicles within the Spitzenkörper can be labelled with the membrane-selective fluorescent dye FM4-64 (Fischer-Parton et al., 2000; Hickey et al., 2005) (Fig. 1.6).

Table 1.3 The timing of the biochemical events leading to conidial germination in $N$. crassa after exposure to media (from Davis, 2000).

\begin{tabular}{c}
\hline O-30 min \\
Hydration \\
Rodlets solubilised \\
Exocellular enzyme released \\
$\gamma$-amino butyrate appears \\
Glutamate degraded \\
Aspartate forms \\
NADP(H) increases \\
Disulphides reduced \\
Heat resistance lost \\
RNA synthesis begins \\
Polysomes assembled \\
\hline 1-2 $\mathrm{h}$ \\
\hline Transport systems increase \\
Minimal amino acid pools appear \\
\hline 2-5 $\mathrm{h}$ \\
\hline Germ tubes emerge \\
\hline Transport further increases \\
\hline DNA synthesis and nuclear divisions occur \\
Oxidative phosphorylation becomes coupled \\
Arginine and ornithine pools enlarge \\
Chitin synthesis begins (ca. 3 hrs) \\
\hline Isoleucine-valine $\mathrm{h}$ \\
\hline Galactosamine polymers appear \\
\hline
\end{tabular}




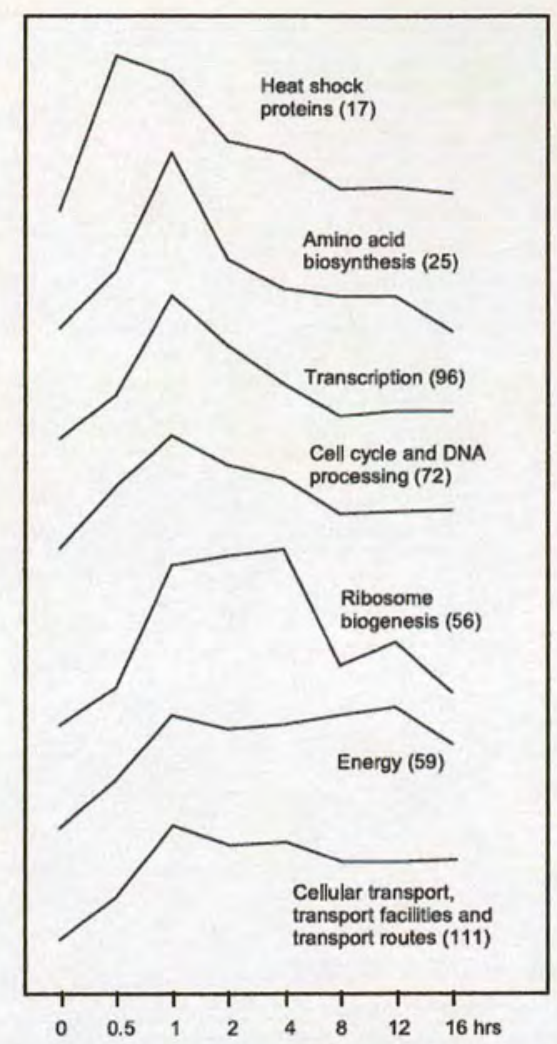

Figure 1.5 Expression profiles of genes in different functional categories during conidial germination. The number of genes in each category are indicated (taken from Kasuga et al., 2005).

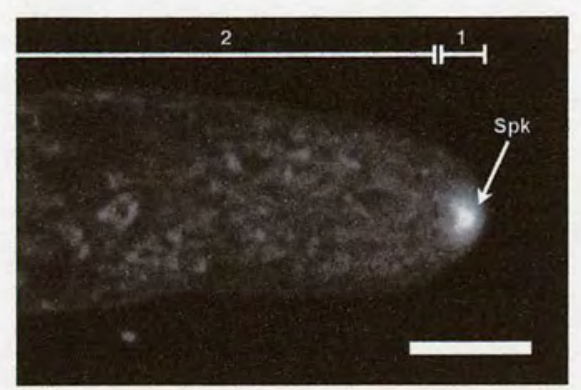

Figure 1.6 A confocal image of a growing hyphal tip of $N$. crassa at the colony periphery after staining with the membrane-selective fluorescent dye FM4-64 (Hickey et al., 2005). Note the concentration of stained secretory vesicles within the Spitzenkörper (Spk), the region of hyphal extension (1), and the non-extending region (2) (from Burnham et al., 2007).

The Spitzenkörper acts as a 'vesicle supply centre' (VSC) (Bartnicki-Garcia, 2002) for the growing hyphal tip. The behaviour of the Spitzenkörper is intimately associated with the pattern of hyphal tip growth and branching (Girbardt, 1957; Bartnicki-Garcia, 2002; Harris and Momany, 2004; Harris et al., 2005). Bartnicki-Garcia et al. (1989; 1995) successfully created a computer simulation of hyphal tip growth in 2D, named the 'VSC model'. The model was later expanded to simulate the process in 3D (Gierz and Bartnicki-Garcia, 2001). 
Secretory vesicles are supplied to the Spitzenkörper from the Golgi, via the cytoskeleton. Microtubules are thought to be involved in the long distance transport and actin microfilaments in the short distance transport of the secretory vesicles (Harris et al., 2005). Vesicles within the Spitzenkörper may also be provided by endocytic recycling (FischerParton et al., 2000). Hyphae also produce satellite Spitzenkörper which are small clusters of vesicles that arise beneath the plasma membrane just behind the main Spitzenkörper (LopezFranco et al., 1994; Lopez-Franco et al., 1995; Fischer-Parton et al., 2000). The fusion of satellite Spitzenkörper with hyphal tips may be responsible for their pulsed growth (LopezFranco et al., 1994).

\subsubsection{Fungal growth forces}

Most work on fungal growth forces has been performed by Nik Money and collaborators (Money, 2001; 2004) and his group was the first to document growth force measurements in the vegetative hyphae of true fungi (Money et al., 2004). Using a miniaturized strain gauge they measured the forces produced by hyphal tips and subsequently calculated the applied pressure (force per unit area, $\mu \mathrm{N} \mu \mathrm{m}^{-2}$ or $\mathrm{MPa}$ ). Pressures ranging from $0.04 \mathrm{MPa}$ (in Sordaria fimicola) to $0.09 \mathrm{MPa}$ (in $N$. crassa) were reported ${ }^{3}$. Penetration by a fungus through its natural environment is also usually facilitated by the fungus secreting a cocktail of digestive enzymes. In fungal plant pathogens, hyphae often develop into specialized infection structures (e.g. on a leaf or root surface) which can generate extremely large forces (turgor pressures of $80 \mathrm{MPa}$ have been measured, Howard et al., 1991; Bechinger et al., 1999) allowing these fungi to efficiently breach the tough host defences. The main contributors to the growth forces of fungal hyphae are believed to be cell turgor and the actin cytoskeleton (Money, 1997; Money, 2001; Money, 2004).

\subsubsection{Woronin bodies and septal pore plugging}

Fungi, growing as a syncytium, allow long range trafficking of organelles and solutes throughout their mycelium through the pores in their septal cross-walls. Accompanying this is the inherent risk of death through the catastrophic loss of cytosol after sustaining cellular damage. A safety measure in this respect is the ability to rapidly occlude the septal pores following damage. This is achieved in most filamentous ascomycete fungi by the septal pore-associated organelle, the Woronin body, named in 1933 by Reginald Buller in honour of Michael Stepanovitch Woronin (Buller, 1933; Trinci and Collinge, 1974; Jedd and Chua,

\footnotetext{
${ }^{3} \mathrm{~A}$ pressure of 1 atmosphere is equivalent to $0.1 \mathrm{MPa}$
} 
2000; Momany et al., 2002; Jedd, 2007). Woronin bodies are formed under the control of polarized gene expression focused towards the hyphal tip (Tey et al., 2005). They are synthesized within the peroxisome to produce an organelle containing a crystallized proteinaceous core composed of HEX-1 protein bounded by a membrane. In $N$. crassa the crystal is observed as a characteristic highly refractive hexagon, $\sim 500 \mathrm{~nm}$ in diameter along its long axis, with a light microscope (Jedd et al., 2000; Yuan et al., 2003). After plugging a septal pore the membrane will reseal and hyphal growth can resume later from this point or elsewhere (Trinci et al., 1974). The mechanisms for initiation and sealing of septal pore plugging by the Woronin body have not yet been elucidated (Tey et al., 2005). Berns et al. (1992) were able to trap Woronin bodies in Nectria haematococca with optical tweezers and draw them away from the septum. Once released the Woronin bodies returned to their original position as the result of some unknown tethering mechanism. Momany et al. (2002) described a filamentous material associated with the Woronin bodies and septal pores, but did not identify its composition.

\subsection{General introduction to the research in this thesis}

The research described in this thesis explores and evaluates the potential of single beam and holographic optical tweezer micromanipulation for use in fungal cell biology research. This is a technique driven body of research which has involved experimental studies into various aspects of fungal cell biology. The aims of the research described in this thesis were to:

- Assess photodamage to conidia caused by optical tweezer trapping

- Develop and improve the CAT homing assay

- Develop methods for producing fixed arrays of spores for future studies on cell-cell communication

- Investigate the tethering of Woronin bodies to septa

- Determine whether ectopically produced intracellular crystals of high refractive index can be used as 'handles' to manipulate intracellular organelles

- Explore how the pattern of hyphal tip growth and branching can be manipulated using either single or holographic optical tweezers

- Determine if optically trapped beads can be used to measure hyphal growth forces

- Determine whether hyphae can be mechanostimulated with trapped beads 
- Demonstrate that trapped porous beads can be used to deliver chemicals to localised regions of hyphae

\subsection{References}

Araujo-Palomares, C.L., Castro-Longoria, E., Riquelme, M., 2007. Ontogeny of the Spitzenkörper in germlings of Neurospora crassa. Fungal Genet. Biol. 44, 492-503.

Arneborg, N., Siegumfeldt, H., Andersen, G.H., Nissen, P., Daria, V.R., Rodrigo, P.J., Gluckstad, J., 2005. Interactive optical trapping shows that confinement is a determinant of growth in a mixed yeast culture. FEMS Microbiol. Lett. 245, 155-159.

Ashkin, A., 1970. Acceleration and trapping of particles by radiation pressure. Phys. Rev. Lett. 24, 156-159.

Ashkin, A., 1991. The study of cells by optical trapping and manipulation of living cells using infrared laser beams. ASGSB. Bull. 4, 133-146.

Ashkin, A., 1997. Optical trapping and manipulation of neutral particles using lasers. Proc. Natl. Acad. Sci. USA 94, 4853-4860.

Ashkin, A., Dziedzic, J.M., 1971. Optical levitation by radiation pressure. Appl. Phys. Lett. 19, 283285.

Ashkin, A., Dziedzic, J.M., 1989. Internal cell manipulation using infrared laser traps. Proc. Natl. Acad. Sci. USA 86, 7914-7918.

Ashkin, A., Dziedzic, J.M., Bjorkholm, J.E., Chu, S., 1986. Observation of a single-beam gradient force optical trap for dielectric particles. Opt. Lett. 11, 288-290.

Ashkin, A., Schutze, K., Dziedzic, J.M., Euteneuer, U., Schliwa, M., 1990. Force generation of organelle transport measured in vivo by an infrared laser trap. Nature 348, 346-348.

Ayano, S., Wakamoto, Y., Yamashita, S., Yasuda, K., 2006. Quantitative measurement of damage caused by 1064-nm wavelength optical trapping of Escherichia coli cells using on-chip single cell cultivation system. Biochem. Biophys. Res. Commun. 350, 678-684.

Bartnicki-Garcia, S., 2002. Hyphal tip growth: outstanding questions. In: Osiewacz, H.D. (Ed.), Molecular Biology of Fungal Development Marcel Dekker, New York, pp. 29-58.

Bartnicki-Garcia, S., Bartnicki, D.D., Gierz, G., Lopez-Franco, R., Bracker, C.E., 1995. Evidence that Spitzenkörper behavior determines the shape of a fungal hypha: a test of the hyphoid model. Exp. Mycol. 19, 153-159.

Bartnicki-Garcia, S., Hergert, F., Gierz, G., 1989. Computer simulation of fungal morphogenesis and the mathematical basis for hyphal tip growth. Protoplasma 153, 46-57.

Baumann, C.G., Bloomfield, V.A., Smith, S.B., Bustamante, C., Wang, M.D., Block, S.M., 2000. Stretching of single collapsed DNA molecules. Biophys. J. 78, 1965-1978.

Bechinger, C., Giebel, K.F., Schnell, M., Leiderer, P., Deising, H.B., Bastmeyer, M., 1999. Optical measurements of invasive forces exerted by appressoria of a plant pathogenic fungus. Science 285, 1896-1899. 
Berns, M.W., Aist, J.R., Wright, W.H., Liang, H., 1992. Optical trapping in animal and fungal cells using a tunable, near-infrared titanium-sapphire laser. Exp. Cell Res. 198, 375-378.

Berns, M.W., Wright, W.H., Wiegand, S.R., 1991. Laser microbeam as a tool in cell biology. Int. Rev. Cytol. 129, 1-44.

Block, S.M., 1990. Optical tweezers: a new tool for biophysics. In: Foster, G.A., Gross, S.P. (Eds.), Noninvasive Techniques in Cell Biology Wiley-Liss, New York, pp. 375-402.

Block, S.M., 1992. Making light work with optical tweezers. Nature 360, 493-495.

Block, S.M., Asbury, C.L., Shaevitz, J.W., Lang, M.J., 2003. Probing the kinesin reaction cycle with a 2D optical force clamp. Proc. Natl. Acad. Sci. USA 100, 2351-2356.

Block, S.M., Blair, D.F., Berg, H.C., 1989. Compliance of bacterial flagella measured with optical tweezers. Nature $338,514-518$.

Block, S.M., Goldstein, L.S., Schnapp, B.J., 1990. Bead movement by single kinesin molecules studied with optical tweezers. Nature $348,348-352$.

Borkovich, K.A., Alex, L.A., Yarden, O., Freitag, M., Turner, G.E., Read, N.D., Seiler, S., BellPedersen, D., Paietta, J., Plesofsky, N., Plamann, M., Goodrich-Tanrikulu, M., Schulte, U., Mannhaupt, G., Nargang, F.E., Radford, A., Selitrennikoff, C., Galagan, J.E., Dunlap, J.C., Loros, J.J., Catcheside, D., Inoue, H., Aramayo, R., Polymenis, M., Selker, E.U., Sachs, M.S., Marzluf, G.A., Paulsen, I., Davis, R., Ebbole, D.J., Zelter, A., Kalkman, E.R., O'Rourke, R., Bowring, F., Yeadon, J., Ishii, C., Suzuki, K., Sakai, W., Pratt, R., 2004. Lessons from the genome sequence of Neurospora crassa: tracing the path from genomic blueprint to multicellular organism. Microbiol. Mol. Biol. Rev. $68,1-108$.

Bracker, C.E., Murphy, D.J., Lopez-Franco, R., 1997. Laser microbeam manipulation of cell morphogenesis in growing fungal hyphae. Proc. SPIE 2983, 67-80.

Buican, T., Smith, M.J., Crissman, H.A., Salzman, G.C., Stewart, C.C., Martin, J.C., 1987. Automated single-cell manipulation and sorting by light trapping. Appl. Opt. 26, 5311-5316.

Buller, A.H.R., 1933. Researches on Fungi, Vol. 5. Longman, London.

Burnham, D.R., Wright, G.D., Read, N.D., McGloin, D., 2007. Holographic and single beam optical manipulation of hyphal tip growth in filamentous fungi. J. Opt. A: Pure Appl. Opt. 9, S172-S179.

Chu, S., 1991. Laser manipulation of atoms and particles. Science 253, 861-866.

Cluzel, P., Lebrun, A., Heller, C., Lavery, R., Viovy, J.L., Chatenay, D., Caron, F., 1996. DNA: an extensible molecule. Science 271, 792-794.

d'Enfert, C., 1997. Fungal spore germination: Insights from the molecular genetics of Aspergillus nidulans and Neurospora crassa. Fungal Genet. Biol. 21, 163-173.

Daria, V.R., Rodrigo, P.J., Gluckstad, J., 2004. Dynamic formation of optically trapped microstructure arrays for biosensor applications. Biosens. Bioelectron. 19, 1439-1444.

Davis, R.H., 2000. Neurospora: Contributions of a model organism. Oxford Univerity Press, Oxford.

Dufresne, E.R., Grier, D.G., 1998. Optical tweezer arrays and optical substrates created with diffractive optics. Rev. Sci. Instrum. 69, 1974-1977. 
Eriksen, R.L., Daria, V.R., Gluckstad, J., 2002a. Fully dynamic multiple-beam optical tweezers. Opt. Exp. 10, 597-602.

Eriksen, R.L., Mogensen, P.C., Gluckstad, J., 2002b. Multiple-beam optical tweezers generated by the generalized phase-contrast method. Opt. Lett. 27, 267-269.

Erlicher, A., Betz, T., Stuhrmann, B., Koch, D., Milner, V., Raizen, M.G., Kas, J., 2002. Guiding neuronal growth with light. Proc. Natl. Acad. Sci. USA 99, 16024-16028.

Finer, J.T., Simmons, R.M., Spudich, J.A., 1994. Single myosin molecule mechanics: piconewton forces and nanometre steps. Nature $368,113-119$.

Fischer-Parton, S., Parton, R.M., Hickey, P.C., Dijksterhuis, J., Atkinson, H.A., Read, N.D., 2000. Confocal microscopy of FM4-64 as a tool for analysing endocytosis and vesicle trafficking in living fungal hyphae. J. Microsc. 198 ( Pt 3), 246-259.

Fleißner, A., Sarkar, S., Jacobson, D.J., Roca, M.G., Read, N.D., Glass, N.L., 2005. The so locus is required for vegetative cell fusion and postfertilization events in Neurospora crassa. Eukaryot. Cell 4, 920-930.

Freitag, M., Hickey, P.C., Raju, N.B., Selker, E.U., Read, N.D., 2004. GFP as a tool to analyze the organization, dynamics and function of nuclei and microtubules in Neurospora crassa. Fungal Genet. Biol. 41, 897-910.

Fuller, D.N., Gemmen, G.J., Rickgauer, J.P., Dupont, A., Millin, R., Recouvreux, P., Smith, D.E., 2006. A general method for manipulating DNA sequences from any organism with optical tweezers. Nucleic Acids Res. 34, e15.

Galagan, J.E., Calvo, S.E., Borkovich, K.A., Selker, E.U., Read, N.D., Jaffe, D., FitzHugh, W., Ma, L.J., Smirnov, S., Purcell, S., Rehman, B., Elkins, T., Engels, R., Wang, S., Nielsen, C.B., Butler, J., Endrizzi, M., Qui, D., Ianakiev, P., Bell-Pedersen, D., Nelson, M.A., Werner-Washburne, M., Selitrennikoff, C.P., Kinsey, J.A., Braun, E.L., Zelter, A., Schulte, U., Kothe, G.O., Jedd, G., Mewes, W., Staben, C., Marcotte, E., Greenberg, D., Roy, A., Foley, K., Naylor, J., Stange-Thomann, N., Barrett, R., Gnerre, S., Kamal, M., Kamvysselis, M., Mauceli, E., Bielke, C., Rudd, S., Frishman, D., Krystofova, S., Rasmussen, C., Metzenberg, R.L., Perkins, D.D., Kroken, S., Cogoni, C., Macino, G., Catcheside, D., Li, W., Pratt, R.J., Osmani, S.A., DeSouza, C.P., Glass, L., Orbach, M.J., Berglund, J.A., Voelker, R., Yarden, O., Plamann, M., Seiler, S., Dunlap, J., Radford, A., Aramayo, R., Natvig, D.O., Alex, L.A., Mannhaupt, G., Ebbole, D.J., Freitag, M., Paulsen, I., Sachs, M.S., Lander, E.S., Nusbaum, C., Birren, B., 2003. The genome sequence of the filamentous fungus Neurospora crassa. Nature 422, 859-868.

Gierz, G., Bartnicki-Garcia, S., 2001. A three-dimensional model of fungal morphogenesis based on the vesicle supply center concept. J. Theor. Biol. 208, 151-164.

Girbardt, M., 1957. Der Spitzenkörper Von Polystictus versicolor (L). Planta 50, 47-59.

Goksor, M., Enger, J., Hanstorp, D., 2004. Optical manipulation in combination with multiphoton microscopy for single-cell studies. Appl. Opt. 43, 4831-4837.

Greulich, K.O., 1999. Micromanipulation by light in biology and medicine: The laser micobeam and optical tweezers. Birkhauser Verlag, Basel, Switzerland.

Greulich, K.O., 2005. Single-molecule studies on DNA and RNA. Chemphyschem. 6, 2458-2471.

Greulich, K.O., Pilarczyk, G., 1998. Laser tweezers and optical microsurgery in cellular and molecular biology. Working principles and selected applications. Cell Mol. Biol. 44, 701-710. 
Greulich, K.O., Pilarczyk, G., Hoffmann, A., Meyer Zu, H.G., Schafer, B., Uhl, V., Monajembashi, S., 2000. Micromanipulation by laser microbeam and optical tweezers: from plant cells to single molecules. J. Microsc. 198, 182-187.

Grier, D.G., Roichman, Y., 2006. Holographic optical trapping. Appl. Opt. 45, 880-887.

Grimbergen, J.A., Visscher, K., Gomes De Mesquita, D.S., Brakenhoff, G.J., 1993. Isolation of single yeast cells by optical trapping. Yeast $9,723-732$.

Grove, S.N., Bracker, C.E., 1970. Protoplasmic organization of hyphal tips among fungi: vesicles and Spitzenkorper. J. Bacteriol. 104, 989-1009.

Harris, S.D., Momany, M., 2004. Polarity in filamentous fungi: moving beyond the yeast paradigm. Fungal Genet. Biol. 41, 391-400.

Harris, S.D., Read, N.D., Roberson, R.W., Shaw, B., Seiler, S., Plamann, M., Momany, C., 2005. Polarisome meets Spitzenkörper: microscopy, genetics, and genomics converge. Eukaryot. Cell 4, 225-229.

Heath, I.B., 1990. Tip growth in plant and fungal cells. Academic Press, San Diego, CA.

Hickey, P.C., Jacobson, D., Read, N.D., Louise Glass, N.L., 2002. Live-cell imaging of vegetative hyphal fusion in Neurospora crassa. Fungal. Genet. Biol. 37, 109-119.

Hickey, P.C., Swift, S.R., Roca, M.G., Read, N.D., 2005. Live-cell imaging of filamentous fungi using vital fluorescent dyes and confocal microscopy. Meth. Microbiol. 34, 63-87.

Howard, R.J., 1981. Ultrastructural analysis of hyphal tip cell growth in fungi: Spitzenkörper, cytoskeleton and endomembranes after freeze-substitution. J. Cell Sci. 48, 89-103.

Howard, R.J., Ferrari, M.A., Roach, D.H., Money, N.P., 1991. Penetration of hard substrates by a fungus employing enormous turgor pressures. Proc. Natl. Acad. Sci. USA 88, 11281-11284.

Jedd, G., 2007. Natural history of the fungal hypha: how Woronin bodies support a multicellular lifestyle. In: Gadd, G.M., Watkinson, S.C., Dyer, P. (Eds.), Fungi in the Environment Cambridge University Press, Cambridge, pp. 22-37.

Jedd, G., Chua, N.H., 2000. A new self-assembled peroxisomal vesicle required for efficient resealing of the plasma membrane. Nat. Cell Biol. 2, 226-231.

Kasuga, T., Townsend, J.P., Tian, C.G., Gilbert, L.B., Mannhaupt, G., Taylor, J.W., Glass, N.L., 2005. Long-oligomer microarray profiling in Neurospora crassa reveals the transcriptional program underlying biochemical and physiological events of conidial germination. Nucleic Acids Res. 33, 6469-6485.

König, K., Liang, H., Berns, M.W., Tromberg, B.J., 1995. Cell-Damage by Near-Ir Microbeams. Nature 377, 20-21.

Kuo, S.C., Sheetz, M.P., 1992. Optical tweezers in cell biology. Trends Cell Biol. 2, 116-118.

Kuo, S.C., Sheetz, M.P., 1993. Force of single kinesin molecules measured with optical tweezers. Science 260, 232-234.

Lafong, A., Hossack, W.J., Arlt, J., Nowakowski, T.J., Read, N.D., 2006. Time-multiplexed LaguerreGaussian holographic optical tweezers for biological applications. Opt. Exp. 14, 3065-3072. 
Lang, M.J., Block, S.M., 2003. Resource letter: LBOT-1: Laser-based optical tweezers. Am. J. Phys. $71,201-215$.

Lang, M.J., Fordyce, P.M., Block, S.M., 2003. Combined optical trapping and single-molecule fluorescence. J. Biol. 2, 6.

Leitz, G., Fallman, E., Tuck, S., Axner, O., 2002. Stress response in Caenorhabditis elegans caused by optical tweezers: Wavelength, power, and time dependence. Biophys. J. 82, 2224-2231.

Leitz, G., Lundberg, C., Fallman, E., Axner, O., Sellstedt, A., 2003. Laser-based micromanipulation for separation and identification of individual Frankia vesicles. FEMS Microbiol. Lett. 224, 97-100.

Leitz, G., Schnepf, E., Greulich, K.O., 1995. Micromanipulation of statoliths in gravity-sensing Chara rhizoids by optical tweezers. Planta 197, 278-288.

Liang, H., Vu, K.T., Krishnan, P., Trang, T.C., Shin, D., Kimel, S., Berns, M.W., 1996. Wavelength dependence of cell cloning efficiency after optical trapping. Biophys. J. 70, 1529-1533.

Liesener, J., Reicherter, M., Haist, T., Tiziani, H.J., 2000. Multi-functional optical tweezers using computer-generated holograms. Opt. Commun. 185, 77-82.

Liu, X., Wang, H., Li, Y., Tang, Y., Liu, Y., Hu, X., Jia, P., Ying, K., Feng, Q., Guan, J., Jin, C., Zhang, L., Lou, L., Zhou, Z., Han, B., 2004. Preparation of single rice chromosome for construction of a DNA library using a laser microbeam trap. J. Biotechnol. 109, 217-226.

Liu, Y., Cheng, D.K., Sonek, G.J., Berns, M.W., Chapman, C.F., Tromberg, B.J., 1995. Evidence for localized cell heating induced by infrared optical tweezers. Biophys. J. 68, 2137-2144.

Lopez-Franco, R., Bartnicki-Garcia, S., Bracker, C.E., 1994. Pulsed growth of fungal hyphal tips. Proc. Natl. Acad. Sci. USA 91, 12228-12232.

Lopez-Franco, R., Bracker, C.E., 1996. Diversity and dynamics of the Spitzenkörper in growing hyphal tips of higher fungi. Protoplasma 195, 90-111.

Lopez-Franco, R., Howard, R.J., Bracker, C.E., 1995. Satellite Spitzenkörper in growing hyphal tips. Protoplasma 188, 85-103.

Mcclure, W.K., Park, D., Robinson, P.M., 1968. Apical Organization in Somatic Hyphae of Fungi. Journal of General Microbiology 50, 177-\&.

Mio, C., Gong, T., Terray, A., Marr, D.W.M., 2000. Design of a scanning laser optical trap for multiparticle manipulation. Rev. Sci. Instrum. 71, 2196-2200.

Mohanty, S.K., Sharma, M., Gupta, P.K., 2006. Generation of ROS in cells on exposure to CW and pulsed near-infrared laser tweezers. Photochem. Photobiol. Sci. 5, 134-139.

Molloy, J.E., Padgett, M.J., 2002. Lights, action: optical tweezers. Contemp. Phys. 43, 241-258.

Momany, M., Richardson, E.A., Sickle, C.V., Jedd, G., 2002. Mapping Woronin body position in Aspergillus nidulans. Mycologia 94, 260-266.

Money, N.P., 2004. The fungal dining habit: a biomechanical perspective. Mycologist 18, 71-76.

Money, N.P., 1997. Wishful thinking of turgor revisited: the mechanics of fungal growth. Fungal Genet. Biol. 21, 173-187. 
Money, N.P., 2001. Biomechanics of invasive hyphal growth. In: Howard, R.J., Gow, N.A.R. (Eds.), The Mycota, Volume III, Biology of the Fungal Cell Springer-Verlag, Berlin, Heidelberg, New York, pp. 3-17.

Money, N.P., Davis, C.M., Ravishankar, J.P., 2004. Biomechanical evidence for convergent evolution of the invasive growth process among fungi and oomycete water molds. Fungal. Genet. Biol. 41, 872876.

Negulescu, P.A., Krasieva, T.B., Khan, A., Kerschbaum, H.H., Cahalan, M.D., 1996. Polarity of T cell shape, motility and sensitivity to antigen. Immunity 4, 421-430.

Neuman, K.C., Block, S.M., 2004. Optical trapping. Rev. Sci. Instrum. 75, 2787-2809.

Neuman, K.C., Chadd, E.H., Liou, G.F., Bergman, K., Block, S.M., 1999. Characterization of photodamage to Escherichia coli in optical traps. Biophys. J. 77, 2856-2863.

Perkins, D.D., Davis, R.H., 2000. Neurospora at the millennium. Fungal Genet. Biol. 31, 153-167.

Peterman, E.J.G., Gittes, F., Schmidt, C.F., 2003. Laser-induced heating in optical traps. Biophys. J. $84,1308-1316$.

Ponelies, N., Scheef, J., Harim, A., Leitz, G., Greulich, K.O., 1994. Laser micromanipulators for biotechnology and genome research. J. Biotechnol. 35, 109-120.

Read, N.D., 2007. Environmental sensing and the filamentous fungal lifestyle. In: Gadd, G.M., Watkinson, S.C., Dyer, P. (Eds.), Fungi in the Environment Cambridge University Press, Cambridge, pp. 38-57.

Read, N.D., Roca, M.G., 2006. Vegetative hyphal fusion in filamentous fungi. In: Baluska, F., Volkmann, D., Barlow, P.W. (Eds.), Cell-Cell Channels Landes Bioscience, Austin, TX.

Roberson, R.W., Fuller, M.S., 1988. Ultrastructural aspects of the hyphal tip of Sclerotium rolfsii preserved by freeze substitution. Protoplasma 146, 143-149.

Roca, M.G., Arlt, J., Jeffree, C.E., Read, N.D., 2005a. Cell biology of conidial anastomosis tubes in Neurospora crassa. Eukaryot. Cell 4, 911-919.

Roca, M.G., Read, N.D., Wheals, A.E., 2005b. Conidial anastomosis tubes in filamentous fungi. FEMS Microbiol. Lett. 249, 191-198.

Sacconi, L., Tolic-Norrelykke, I.M., Stringari, C., Antolini, R., Pavone, F.S., 2005. Optical micromanipulations inside yeast cells. Appl. Opt. 44, 2001-2007.

Schmit, J.C., Brody, S., 1976. Biochemical genetics of Neurospora crassa conidial germination. Bacteriol. Rev. 40, 1-41.

Sheetz, M.P., 1998. Laser Tweezers in Cell Biology. Meth. Cell Biol. 55.

Stevenson, D.J., Lake, T.K., Agate, B., Garces-Chavez, V., Dholakia, K., Gunn-Moore, F., 2006. Optically guided neuronal growth at near infrared wavelengths. Opt. Exp. 14, 9786-9793.

Svoboda, K., Block, S.M., 1994. Biological applications of optical forces. Annu. Rev. Biophys. Biomol. Struct. 23, 247-285.

Tey, W.K., North, A.J., Reyes, J.L., Lu, Y.F., Jedd, G., 2005. Polarized gene expression determines Woronin body formation at the leading edge of the fungal colony. Mol. Biol. Cell 16, 2651-2659. 
Tolic-Norrelykke, I.M., Munteanu, E.L., Thon, G., Oddershede, L., Berg-Sorensen, K., 2004. Anomalous diffusion in living yeast cells. Phys. Rev. Lett. 93, 078102.

Tolic-Norrelykke, I.M., Sacconi, L., Stringari, C., Raabe, I., Pavone, F.S., 2005. Nuclear and divisionplane positioning revealed by optical micromanipulation. Curr. Biol. 15, 1212-1216.

Trinci, A.P., Collinge, A.J., 1974. Occlusion of the septal pores of damaged hyphae of Neurospora crassa by hexagonal crystals. Protoplasma 80, 57-67.

Virag, A., Harris, S.D., 2006. The Spitzenkörper: a molecular perspective. Mycol. Res. 110, 4-13.

Visscher, K., Gross, S.P., Block, S.M., 1996. Construction of multiple-beam optical traps with nanometer-level position sensing. IEEE J. Sel. Top. Quant. Electron. 2, 1066-1076.

Wang, M.D., Schnitzer, M.J., Yin, H., Landick, R., Gelles, J., Block, S.M., 1998. Force and velocity measured for single molecules of RNA polymerase. Science 282, 902-907.

Wang, M.D., Yin, H., Landick, R., Gelles, J., Block, S.M., 1997. Stretching DNA with optical tweezers. Biophys. J. 72, 1335-1346.

Wang, Y., Botvinick, E.L., Zhao, Y., Berns, M.W., Usami, S., Tsien, R.Y., Chien, S., 2005. Visualizing the mechanical activation of Src. Nature 434, 1040-1045.

Weber, G., Greulich, K.O., 1992. Manipulation of cells, organelles, and genomes by laser microbeam and optical trap. Int. Rev. Cytol. 133, 1-41.

Wei, X., Tromberg, B.J., Cahalan, M.D., 1999. Mapping the senesitivity of T cells with an optical trap: Polarity and minimal number of reseptors for $\mathrm{Ca}^{2+}$ signaling. Proc. Natl. Acad. Sci. U. S. A 96, 8471-8476.

Wright, G.D., Arlt, J., Poon, W.C., Read, N.D., 2007a. Optical tweezer micromanipulation of filamentous fungi. Fungal. Genet. Biol. 44, 1-13.

Wright, G.D., Arlt, J., Poon, W.C.K., Read, N.D., 2005. Measuring fungal forces with optical tweezers. Proc. SPIE 5930, F1-F7.

Wright, G.D., Arlt, J., Poon, W.C.K., Read, N.D., 2007b. Experimentally manipulating fungi with optical tweezers. Mycoscience 48, 15-19.

Wright, W.H., Sonek, G.J., Tadir, Y., Berns, M.W., 1990. Laser trapping in cell biology. J. Quant. Elec. 26, 2148-2157.

Yuan, P., Jedd, G., Kumaran, D., Swaminathan, S., Shio, H., Hewitt, D., Chua, N.H., Swaminathan, K., 2003. A HEX-1 crystal lattice required for Woronin body function in Neurospora crassa. Nat. Struct. Biol. 10, 264-270 


\section{Chapter 2 - Materials and methods}

\subsection{Chemicals}

Unless stated otherwise chemicals were purchased from Sigma or Fluka (both at www.sigmaaldrich.com).

\subsection{Fungal species and strains}

Experiments were performed using the fungal species and strains in Table 2.1.

Table 2.1 Species and strains used.

\begin{tabular}{|c|c|c|c|}
\hline Species & Strain & Genotype & Source \\
\hline \multirow[t]{3}{*}{ Neurospora crassa } & $\begin{array}{l}\text { Wild type } \\
\text { N2489 }\end{array}$ & Mat A 74-OR23-IV & FGSC \\
\hline & $\begin{array}{l}\text { N2276-1 } \\
\text { (H1-GFP) }\end{array}$ & $\begin{array}{l}\text { Mat A his- } 3^{+}:: P c c g-1- \\
h H 1^{+}-\text {sgfp }^{+}\end{array}$ & $\begin{array}{l}\text { M. Frietag, Oregon } \\
\text { State University, USA }\end{array}$ \\
\hline & mak-2-GFP & 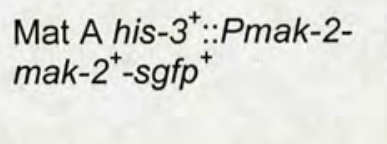 & $\begin{array}{l}\text { A. Fleißner \& L. Glass, } \\
\text { University of California, } \\
\text { Berkeley, USA }\end{array}$ \\
\hline Aspergillus nidulans & SDV38 & alcA::GFP::HexA & $\begin{array}{l}\text { D. Veith \& R. Fischer, } \\
\text { University of Karlsruhe, } \\
\text { Germany }\end{array}$ \\
\hline $\begin{array}{l}\text { Schizosaccharomyces } \\
\text { pombe }\end{array}$ & SO1898 & $\begin{array}{l}\text { pREP1- } \\
\text { polyhedrin/UCH2::GFP }\end{array}$ & $\begin{array}{l}\text { S. Oliferenko \& G. } \\
\text { Jedd, Temasek Life } \\
\text { Sciences Laboratory, } \\
\text { Singapore }\end{array}$ \\
\hline
\end{tabular}

\subsection{Culture media and growth conditions}

Species were maintained and stored on the appropriate solid agar media, except Schizosacc. pombe which was maintained in liquid culture. Agar (Oxoid agar No. 3, www.oxoid.com) was used at an increased concentration of $2 \%(\mathrm{w} / \mathrm{v})$ to produce a firmer gel, encouraging growth to be limited to one plane on the agar surface, which is more suitable for light microscopy. Agar media were prepared in $300 \mathrm{ml}$ batches contained in $500 \mathrm{ml}$ flasks, and sterilised by autoclaving for $15-30 \mathrm{~min}$ at $121^{\circ} \mathrm{C}$. They were then either allowed to set for storage, or when cooled to $50^{\circ} \mathrm{C}$ poured into plastic Petri dishes $(8.5 \mathrm{~cm}$ diam., Greiner BioOne, www.greinerbioone.com) or made up as slants in plastic tubes ( $3 \mathrm{ml}$ agar in $15 \mathrm{ml}$ Greiner tubes, allowed to set at $>45^{\circ}$, Greiner Bio-One www.greinerbioone.com) with plastic screw caps. If stored the agar was melted by microwaving $(650 \mathrm{~W})$ set at medium 
power for 10-15 min, swirling every $3 \mathrm{mins}$, then allowed to cool to $50{ }^{\circ} \mathrm{C}$ in a water bath before pouring. Once poured plates and tubes were stored in the dark, at room temperature to prevent condensation, and used within 1 month. Liquid media (for microscopy preparation and dilution of dyes) were prepared in $100 \mathrm{ml}$ batches to the same recipes, excluding agar, and stored in $10 \mathrm{ml}$ aliquots in plastic tubes $\left(15 \mathrm{ml}\right.$ Greiner tubes) in the refrigerator at $4{ }^{\circ} \mathrm{C}$ (importantly, they were allowed to warm to room temperature before use).

\subsubsection{Culturing Neurospora crassa}

All $N$. crassa strains were grown on Vogel's medium (Section 2.3.1.1) at $25{ }^{\circ} \mathrm{C}$ in continuous light unless otherwise stated. For experiments requiring hyphal tips Petri dishes were inoculated to one side of the plate with either an agar plug from an established colony or a $10 \mu \mathrm{l}$ drop of a dense conidial suspension. The plate was then sealed with Parafilm (www.parafilm.com) before being placed in the incubator. To enable faster growth Parafilm could be replaced with Micropore tape (3M, www. 3 m.com), enabling better aeration, and for containment the plate was placed within a larger plastic Petri dish $(14 \mathrm{~cm}$ diam., Nunc, www.nuncbrand.com). For experiments requiring spores agar slants were inoculated with a drop $(\sim 20 \mu$ l) of a dense conidial suspension, the caps were then loosened to allow aeration and secured with Micropore tape.

\subsubsection{Neurospora crassa culture media}

Solid Vogel's medium was prepared according to Table 2.2. Liquid Vogel's medium followed the same recipe, excluding the agar.

Table 2.2 Vogel's sucrose minimal medium.

\begin{tabular}{cc}
\hline Component & Quantity \\
\hline *Vogels 50* stock solution & $20 \mathrm{ml}$ \\
Sucrose & $20 \mathrm{~g}$ \\
Agar & $20 \mathrm{~g}$ \\
$\mathrm{dH}_{2} \mathrm{O}$ & $1 \mathrm{I}$ \\
\hline
\end{tabular}

*see Table 2.3 
Table 2.3 Composition of Vogel's $50 \mathrm{x}$ stock solution (stored at $4{ }^{\circ} \mathrm{C}$ ).

\begin{tabular}{cc}
\hline Component & $\begin{array}{c}\text { Quantity per litre } \\
\mathbf{d H}_{2} \mathrm{O}\end{array}$ \\
\hline $\mathrm{Na}_{3}$ Citrate- $2 \mathrm{H}_{2} \mathrm{O}$ & $126.7 \mathrm{~g}$ \\
$\mathrm{KH}_{2} \mathrm{PO}_{4}$ & $250.0 \mathrm{~g}$ \\
$\mathrm{NH}_{4} \mathrm{NO}_{3}$ & $100.0 \mathrm{~g}$ \\
$\mathrm{MgSO}_{4}-7 \mathrm{H}_{2} \mathrm{O}$ & $10.0 \mathrm{~g}$ \\
$\mathrm{CaCl}_{2}-2 \mathrm{H}_{2} \mathrm{O}$ & $5.0 \mathrm{~g}$ \\
**Trace elements solution & $5 \mathrm{ml} \mathrm{stock}$ \\
†Biotin solution & $5 \mathrm{ml}$ stock \\
Chloroform & $2-3 \mathrm{ml}$ \\
\hline
\end{tabular}

** see Table $2.4, \dagger$ see Table 2.5

Table 2.4 Composition of Vogel's trace elements stock solution (stored at $4{ }^{\circ} \mathrm{C}$ ).

\begin{tabular}{cc}
\hline Component & Quantity per litre $\mathrm{dH}_{2} \mathrm{O}$ \\
\hline Citric acid-1 $\mathrm{H}_{2} \mathrm{O}$ & $5 \mathrm{~g}$ \\
$\mathrm{ZnSO}_{4}-7 \mathrm{H}_{2} \mathrm{O}$ & $5 \mathrm{~g}$ \\
$\mathrm{Fe}\left(\mathrm{NH}_{4}\right)_{2} \mathrm{SO}_{4}-7 \mathrm{H}_{2} \mathrm{O}$ & $1 \mathrm{~g}$ \\
$\mathrm{CuSO}_{4}-5 \mathrm{H}_{2} \mathrm{O}$ & $0.25 \mathrm{~g}$ \\
$\mathrm{MnSO}_{4}-1 \mathrm{H}_{2} \mathrm{O}^{*}$ & $0.05 \mathrm{~g}$ \\
$\mathrm{H}_{2} \mathrm{BO}_{4}$ & $0.05 \mathrm{~g}$ \\
$\mathrm{Na}_{2} \mathrm{MoO}_{4}-2 \mathrm{H}_{2} \mathrm{O}$ & $0.05 \mathrm{~g}$ \\
\hline
\end{tabular}

Table 2.5 Composition of biotin stock solution for Vogel's (stored at $4^{\circ} \mathrm{C}$ ).

\begin{tabular}{cc}
\hline Component & Content per litre $\mathrm{dH}_{2} \mathrm{O}$ \\
\hline d-biotin & $5 \mathrm{~g}$ \\
\hline
\end{tabular}




\subsubsection{Neurospora crassa storage}

Agar slants were inoculated and allowed to grow at $25^{\circ} \mathrm{C}$ for 7 days in continuous light (Sanyo MIR-153 incubator illuminated by a single $15 \mathrm{~W}$ 'warm white' fluorescent tube light, www.sanyo-biomedical.co.uk) by which time significant conidiation had occurred. These slants were then refrigerated at $4^{\circ} \mathrm{C}$ and used for up to 4 weeks. Additionally similar slants were frozen at $-20{ }^{\circ} \mathrm{C}$ as a backup for several years. To recover the cultures an inoculating loop was used to remove spores and transfer them into liquid media or onto slants.

\subsection{Silica gel for long term storage of conidia}

For long term storage conidia were stored on silica gel (Davis and de Serres, 1970; Smith and Oinons, 1983; Stock Preservation Protocol, FGSC, www.fgsc.net/Neurospora/NeurosporaProtocolGuide.htm). Silica gel (1-3 mm particle size) was sterilised at $180{ }^{\circ} \mathrm{C}$ for $1.5 \mathrm{~h}$ and after cooling was used to half fill sterile $20 \mathrm{ml}$ universal glass vials sealed with polypropylene screw caps. The vials were closed and cooled on ice, prior to adding the spores, to prevent heat damage of the spores by the resultant exothermic reaction. $2 \mathrm{ml}$ of sterilised $7 \% \mathrm{w} / \mathrm{v}$ non-fat dried milk (Marvel, UK) was added to a conidiating slant of $N$. crassa and swirled to produce a dense spore suspension. Into each of the vials containing silica gel $500 \mu$ of the resultant spore suspension was added, then with the lid on shaken to distribute the spores evenly and returned to the ice to prevent further heating. The vials were then stored in the dark at room temperature and have been reported to be useful for 30 years (Stock Preservation Protocol, FGSC, www.fgsc.net/Neurospora/NeurosporaProtocolGuide.htm). To recover the culture a few silica gel particles were removed and used to inoculate a fresh slant.

\subsection{Cryopreservation for long term storage}

A second method for long term storage was also used. Agar plugs $\left(\sim 5 \mathrm{~mm}^{3}\right)$ from conidiating plates were added to $1 \mathrm{ml}$ of a $15 \% \mathrm{v} / \mathrm{v}$ glycerol in Vogel's liquid medium in Eppendorf tubes. The tubes were sealed then vortexed on a Whirlimixer to disperse the spores throughout the solution, then snap frozen by immersing them in liquid nitrogen before being moved to the $-80^{\circ} \mathrm{C}$ freezer. To recover the cultures the Eppendorf was allowed to thaw and a sample of the solution added to a plate or slant. 


\subsubsection{Culturing Aspergillus nidulans}

The A. nidulans strain used here has HexA (short for Hexagonal peroxisome) tagged with GFP under the control of the alcA promoter, producing fluorescent Woronin bodies under inducing conditions (i.e. in the presence of ethanol or glycerol). Aspergillus nidulans was grown on Aspergillus Complete Medium plates (AspCM; Section 2.3.2.1.) at $37{ }^{\circ} \mathrm{C}$ in continuous light. For induction of the alcA promoter $400 \mu \mathrm{l}$ of filter sterilized $1 \%$ ethanol solution was spread over the agar and allowed to soak in before inoculation. Petri dishes were inoculated to one side of the plate with either an agar plug from an established colony or a $10 \mu \mathrm{l}$ drop of spore suspension. The plate was then sealed with Parafilm (www.parafilm.com) before being placed in the incubator.

\subsubsection{Aspergillus nidulans culture media}

Solid AspCM was prepared according to Table 2.6. Liquid AspCM followed the same recipe, excluding the agar.

Table 2.6 Aspergillus Complete Medium (AspCM).

\begin{tabular}{cc}
\hline Component & Quantity per litre $\mathrm{dH}_{2} \mathrm{O}$ \\
\hline$* 20 \times$ Nitrate Salts & $50 \mathrm{ml}$ \\
Maltose & $20 \mathrm{~g}$ \\
Peptone & $2 \mathrm{~g}$ \\
Yeast extract & $1 \mathrm{~g}$ \\
Casein enzymic hydrosylate & $1 \mathrm{~g}$ \\
$\star * 1000 \times$ Trace elements & $1 \mathrm{ml}$ \\
$+V i t a m i n$ solution & $1 \mathrm{ml}$ \\
Agar & $20 \mathrm{~g}$ \\
\hline
\end{tabular}

Adjusted to $\mathrm{pH} 6.5$ using $1 \mathrm{M} \mathrm{NaOH}$

* see Table 2.7, ${ }^{* *}$ see Table $2.8, \dagger$ see Table 2.9 
Table 2.7 Composition of AspCM 20x Nitrate salt solution (stored at $4{ }^{\circ} \mathrm{C}$ ).

\begin{tabular}{cc}
\hline Component & Quantity per litre $\mathrm{dH}_{2} \mathrm{O}$ \\
\hline $\mathrm{NaNO}_{3}$ & $120 \mathrm{~g}$ \\
$\mathrm{KCL}$ & $10.4 \mathrm{~g}$ \\
$\mathrm{MgSO}_{4}{ }^{*} 7 \mathrm{H}_{2} \mathrm{O}$ & $10.4 \mathrm{~g}$ \\
$\mathrm{KH}_{2} \mathrm{PO}_{4}$ & $30.4 \mathrm{~g}$ \\
\hline
\end{tabular}

Table 2.8 Composition of AspCM trace elements solution (stored at $4^{\circ} \mathrm{C}$ ).

\begin{tabular}{cc}
\hline Component & Quantity \\
\hline $\mathrm{dH}_{2} \mathrm{O}$ & $60 \mathrm{ml}$ \\
$\mathrm{ZnO}_{4}-7 \mathrm{H}_{2} \mathrm{O}$ & $2.2 \mathrm{~g}$ \\
$\mathrm{H}_{3} \mathrm{BO}_{3}$ & $1.1 \mathrm{~g}$ \\
$\mathrm{MnCl}_{2}-4 \mathrm{H}_{2} \mathrm{O}$ & $0.5 \mathrm{~g}$ \\
$\mathrm{FeSO}_{4}-7 \mathrm{H}_{2} \mathrm{O}$ & $0.5 \mathrm{~g}$ \\
$\mathrm{CoCl}_{2}-6 \mathrm{H}_{2} \mathrm{O}$ & $0.17 \mathrm{~g}$ \\
$\mathrm{CuSO}_{4}-5 \mathrm{H}_{2} \mathrm{O}$ & $0.16 \mathrm{~g}$ \\
$\mathrm{Na}_{2} \mathrm{MoO}_{4}-2 \mathrm{H}_{2} \mathrm{O}$ & $0.15 \mathrm{~g}$ \\
$\mathrm{Na}_{4} \mathrm{EDTA}^{\mathrm{N}}$ & $5 \mathrm{~g}$ \\
\hline
\end{tabular}

Boiled, cooled to $60^{\circ} \mathrm{C}$, adjusted to $\mathrm{pH} 6.5$ with $\mathrm{KOH}$ and cooled to room temperature. Volume adjusted to $100 \mathrm{ml}$ with $\mathrm{dH}_{2} \mathrm{O}$. Precipitate resuspended before use.

\subsubsection{Aspergillus nidulans storage}

To store $A$. nidulans cryopreservation was used. Agar plugs $\left(\sim 5 \mathrm{~mm}^{3}\right)$ from sporulating plates were added to $1 \mathrm{ml}$ of a $15 \% \mathrm{v} / \mathrm{v}$ glycerol in AspCM liquid medium in Eppendorf tubes. The tubes were sealed then vortexed on a Whirlimixer to disperse the spores throughout the solution, then snap frozen by immersing them liquid nitrogen before being moved to the $-80^{\circ} \mathrm{C}$ freezer. To recover the cultures the Eppendorf was allowed to thaw and a sample of the solution was spread over a plate. 
Table 2.9 Composition of AspCM vitamin solution (stored in the dark at $4^{\circ} \mathrm{C}$ ).

\begin{tabular}{cc}
\hline Component & Quantity \\
\hline Biotin & $100 \mathrm{mg}$ \\
Pyridoxin & $100 \mathrm{mg}$ \\
Thiamine & $100 \mathrm{mg}$ \\
Riboflavin & $100 \mathrm{mg}$ \\
PABA (p-aminobenzoic & $100 \mathrm{mg}$ \\
acid) & $100 \mathrm{mg}$ \\
Nicotinic acid & $2 \mathrm{drops}$ \\
\hline
\end{tabular}

\subsubsection{Culturing Schizosaccharomyces pombe}

The Schizosacc. pombe strain used here had Uch2 tagged with GFP to fluorescently label the nuclear envelope, and also produced polyhedrin, which forms large crystals within the nuclei. Schizosaccharomyces pombe was grown in continuously lit shake culture at $24{ }^{\circ} \mathrm{C}$, in conical flasks sealed with sponge plugs, in Edinburgh Minimal Media (EMM; section 2.3.3.1) supplemented with leucine and thiamine as required for repression or activation of the transgenic genes. Subculturing was on EMM -leu +thi. The lack of leucine ensured the media was selective to prevent the loss of the plasmid containing the transgenic genes. Thiamine, at $15 \mu \mathrm{M}$ concentration, was present to repress the $n m t 1$ promoter preventing the production of polyhedrin, the crystals of which perturb normal cell replication.

\subsubsection{Schizosaccharomyces pombe culture media}

Liquid EMM media was prepared according to Table 2.10. To make solid media for plates $2 \%$ Difco Bacto (www.bacto.com.au), agar was added prior to autoclaving. 
Table 2.10 Edinburgh minimal medium (EMM).

\begin{tabular}{cc}
\hline Component & Quantity per litre $\mathrm{dH}_{2} \mathrm{O}$ \\
\hline Potassium hydrogen phthalate & $3 \mathrm{~g}$ \\
$\mathrm{Na}_{2} \mathrm{HPO}_{4}$ & $2.2 \mathrm{~g}$ \\
$\mathrm{NH}_{4} \mathrm{Cl}$ & $5 \mathrm{~g}$ \\
Glucose & $20 \mathrm{~g}$ \\
${ }^{*} \mathrm{EMM} 50 \times$ salts & $20 \mathrm{ml}$ \\
**EMM vitamins & $1 \mathrm{ml}$ \\
$\dagger \mathrm{H}$ EMM minerals & $0.1 \mathrm{ml}$ \\
Supplements & $225 \mathrm{mg}$ each \\
\hline
\end{tabular}

${ }^{*}$ see Table 2.11, ${ }^{\star \star}$ see Table 2.12, †see Table 2.13

Table 2.11 Composition of EMM $50 \times$ salts solution (filter sterilized and stored at $4{ }^{\circ} \mathrm{C}$ ).

\begin{tabular}{cc}
\hline Component & Quantity per litre $\mathrm{dH}_{2} \mathrm{O}$ \\
\hline $\mathrm{MgCl}_{2}-6 \mathrm{H}_{2} \mathrm{O}$ & $52.5 \mathrm{~g}$ \\
$\mathrm{CaCl}_{2}-2 \mathrm{H}_{2} \mathrm{O}$ & $0.735 \mathrm{~g}$ \\
$\mathrm{KCl}$ & $50 \mathrm{~g}$ \\
$\mathrm{Na}_{2} \mathrm{SO}_{4}$ & $2 \mathrm{~g}$ \\
\hline
\end{tabular}

Table 2.12 Composition of EMM 1000x vitamin stock solution (filter sterilized and stored at $\left.4{ }^{\circ} \mathrm{C}\right)$.

\begin{tabular}{cc}
\hline Component & Quantity per litre $\mathrm{dH}_{2} \mathrm{O}$ \\
\hline Pantothenic acid & $1 \mathrm{~g}$ \\
Nicotininc acid & $10 \mathrm{~g}$ \\
Inositol & $10 \mathrm{~g}$ \\
Biotin & $10 \mathrm{mg}$ \\
\hline
\end{tabular}


Table 2.13 Composition of EMM 10,000 $\times$ mineral solution (filter sterilized and stored at $\left.4{ }^{\circ} \mathrm{C}\right)$.

\begin{tabular}{cc}
\hline Component & $\begin{array}{c}\text { Quantity per litre } \\
\mathbf{d ~}_{2} \mathrm{O}\end{array}$ \\
\hline Boric acid & $5 \mathrm{~g}$ \\
MNSO4 & $4 \mathrm{~g}$ \\
$\mathrm{ZnSO} 4-7 \mathrm{H} 2 \mathrm{O}$ & $4 \mathrm{~g}$ \\
$\mathrm{FeCl} 2-6 \mathrm{H} 2 \mathrm{O}$ & $2 \mathrm{~g}$ \\
Molybdic acid & $0.4 \mathrm{~g}$ \\
$\mathrm{KI}$ & $1 \mathrm{~g}$ \\
CuSO4-5H2O & $0.4 \mathrm{~g}$ \\
Citric acid & $10 \mathrm{~g}$ \\
\hline
\end{tabular}

\subsubsection{Schizosaccharomyces pombe storage}

Cryopreservation was used to store $S$. pombe. 24 hour old liquid cultures were put in Eppendorf tubes then glycerol added to $50 \% \mathrm{v} / \mathrm{v}$. Tubes were sealed and vortexed to mix the solutions then snap frozen by immersing in liquid nitrogen before being stored in the $-80{ }^{\circ} \mathrm{C}$ freezer. To recover the culture the Eppendorf was allowed to thaw and a sample of the solution used to inoculate fresh liquid media.

\subsection{Preparation for microscopy}

For experiments requiring $N$. crassa hyphal tips a mycelium was allowed to grow $3-5 \mathrm{~cm}$ across the Petri dish ( $N$. crassa would be inoculated 18-24 hours prior to when needed). Either the inverted agar block method or sandwich culture was employed for microscopic imaging (see section 2.4 .1 and 2.4 .2 respectively).

When $N$. crassa conidia or germlings were required slants were grown for $\sim 7$ days. A spore suspension was then made by adding $1 \mathrm{ml}$ of Vogel's medium to the slant, swirling and then aspirating. To break up chains of macroconidia, the spore suspension was vortexed in an Eppendorf tube. If necessary, the spore suspension was then filtered through a pipette tip containing a ball of cotton wool to remove hyphal fragments and the larger arthroconidia. The concentration of conidia was then determined using a Fuchs-Rosenthal haemocytometer (Paul Marienfeld GmbH \& Co. KG, www.marienfeld-superior.com) and adjusted by dilution 
in Vogel's to $1 \times 10^{6}$ spores $\mathrm{ml}^{-1}$. The conidial suspension was then used as described in section 2.4.3.

For experiments with $A$. nidulans a spore suspension was made by adding sterile $\mathrm{dH}_{2} \mathrm{O}$ to a week old sporulating plate, agitating with a sterile glass rod and then aspirating. Refrigeration at $4{ }^{\circ} \mathrm{C}$ allowed these spores to be used for up to 2 weeks. The spore solution was then used to inoculate solid medium by spreading, with a flamed glass spreader, and spores allowed to germinate overnight at room temperature for use in experiments. The inverted agar block method (section 2.4.1) was then employed.

For $S$. pombe experiments it was necessary to promote the production of the polyhedrin crystals. For this EMM -leu -thi media was inoculated and the cells allowed to grow in shake culture at $24^{\circ} \mathrm{C}$ for $>18$ hours. $300 \mu$ lof the cell suspension was then transferred to a EMM -leu -thi solid media Petri dish and spread using a flamed glass spreader. 10 min was allowed for the excess liquid to be soaked up by the agar and then samples prepared using the inverted agar block method (section 2.4.1).

\subsubsection{Inverted agar block culture}

Using a sterile scalpel a section of agar measuring $\sim 2 \mathrm{~cm} \times 2 \mathrm{~cm}$ was excised from the colony periphery, such that the leading hyphal tips covered half of the agar block. The agar was then inverted and transferred onto a droplet of liquid media on a large (48 x $64 \mathrm{~mm} \# 1.5$, R.A. Lamb, www.ralamb.co.uk) cleaned glass coverslip (Fig. 2.1). During the preparation the hyphae can suffer perturbation which can cause a cessation of growth and subsequent apical branching. For this reason the sample was placed on the microscope stage and allowed $>30 \mathrm{~min}$ to recover and resume normal growth. When using a sample over extended periods of time drying could be a problem. To prevent this, the base from a plastic Petri dish, with a hole cut through the centre so as not to perturb the illumination, containing a damp piece of filter paper was placed over the sample on the microscope stage. From time to time the liquid media was also refreshed from the side using a pipette.

The technique was modified, when extra room beneath the agar was required (e.g. for manipulation of beads), by supporting the agar on each side using strips of adhesive electricians insulation tape (Fig. 2.2). To enhance the adherence of hyphae to the coverglass cleaned coverslips were coated with poly-L-lysine (section 2.4.5). Alternatively, the sandwich culture method could also be used (section 2.4.2). 


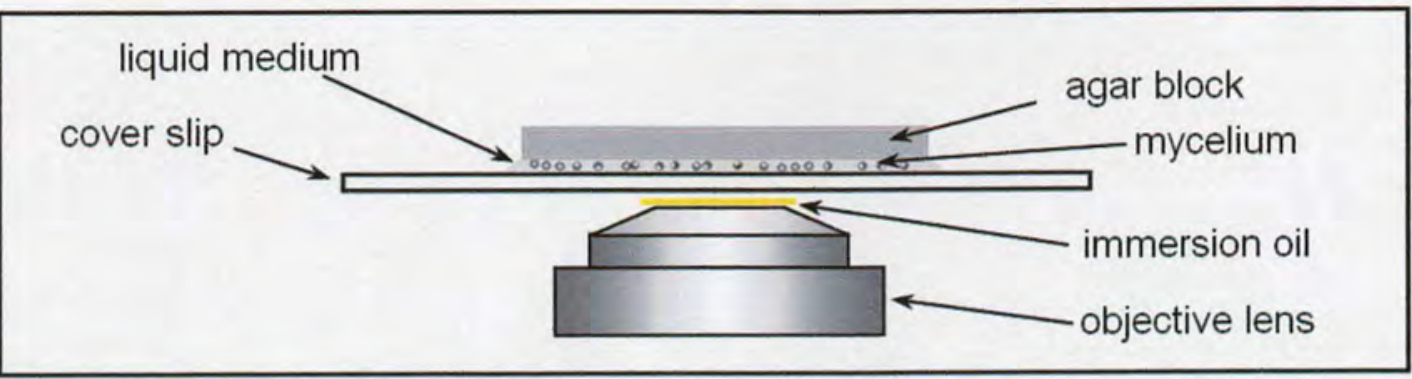

Figure 2.1 Inverted agar block culture method (adapted from Hickey et al., 2005).

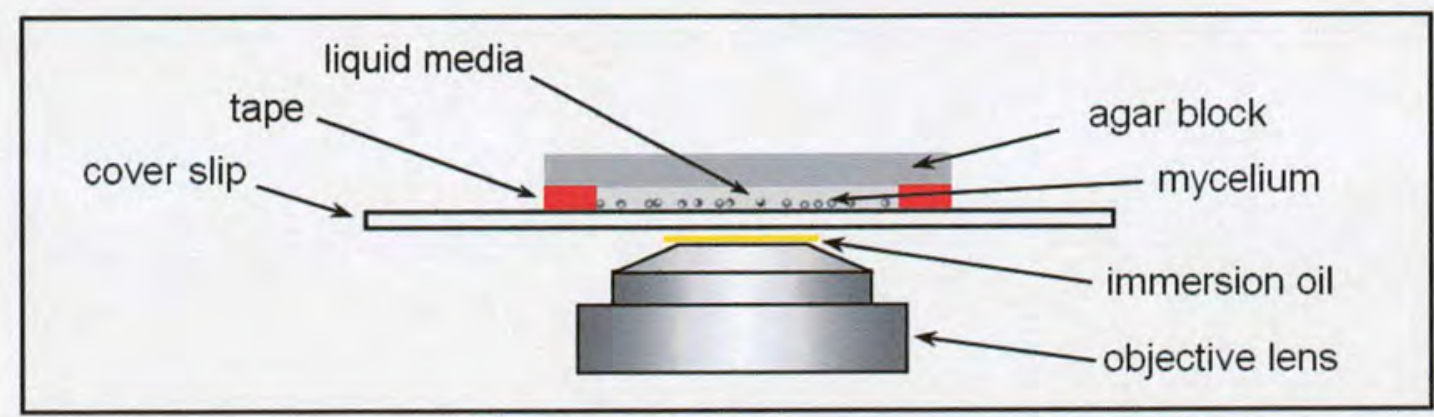

Figure 2.2 Modified inverted agar block method, using adhesive electricians insulation tape to raise the level of the agar providing extra room.

\subsubsection{Sandwich culture}

The agar on a Petri dish was first covered with a sterilised disc of cellophane (525 gauge uncoated rayophane, A.A. Packaging, www.aapackaging.co.uk) onto which fungal material was inoculated and grown as normal. Sandwich slide chambers were then prepared to a similar specification as previously described (Lopez-Franco and Bracker, 1996) (Fig 2.3). Strips of adhesive electricians insulation tape were placed on a large coverslip to support a smaller coverslip ( $22 \times 40 \mathrm{~mm} \# 1.5$, Menzel-Gläser, www.menzel.de). A piece of the cellophane with hyphae growing on top was excised from the Petri dish and transferred to a droplet of liquid media on the lower coverslip, between the two strips of tape. The mycelium was then floated off the cellophane, which was subsequently removed, and the upper coverslip was carefully mounted on top. Care had to be taken during the preparation to prevent excessive damage to the hyphae. To enhance the adherence of hyphae to the lower coverglass cleaned coverslips were coated with poly-L-lysine (section 2.4.5). 


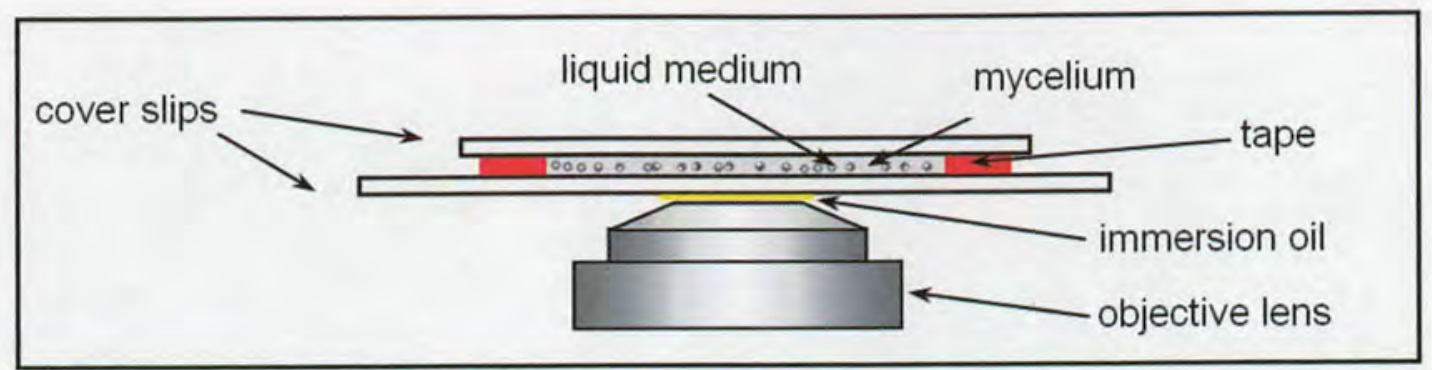

Figure 2.3 Sandwich culture method.

\subsubsection{Liquid culture}

Liquid culture techniques were commonly used in experiments with conidia and germlings. A $1 \times 10^{6}$ spores $\mathrm{ml}^{-1}$ solution was prepared (section 2.4) and used in a number of ways, depending on the experimental requirements: (1) $200 \mu \mathrm{l}$ droplets of the spore solution were placed into eight well slide culture chambers (Nalge Nunc International, www.nalgenunc.com), (2) dropets of the spore solution were placed onto coverlips coated with poly (vinyl) alcohol (PVA) to prevent the conidia adhering to the glass (Krylov and Dovichi, 2000) and overlaid with a second coverslip (see section 2.4.5), or (3) droplets of the spore solution were placed into custom-built sample chambers (described in more detail in sections 3.2.3 and 3.2.4).

For experiments involving Cygel ${ }^{\mathrm{TM}}$ (Biostatus Ltd., www.biostatus.com), a 70\% v/v solution of Cygel in Vogels liquid medium was used. Cygel is a thermoreversible gel that is a liquid when cold and a gel when warmed. The Cygel, all pipette tips, Eppendorf tubes and the 8 well slide culture chambers were kept on an ice pack to prevent warming, and therefore gelation, during sample preparation. A concentrated spore solution in Vogel's was used to dilute the Cygel to result in the desired spore and Cygel concentrations. A heating stage (Reichert, www.reichert.com) was mounted on the microscope stage for these experiments to allow the necessary temperature shifts to be made.

\subsubsection{Microfluidic cells}

Microfluidic cells, or flow cells, were fabricated (by Andy Garrie, to Emmelyn Graham's design) from Perspex and the Y-shaped channel milled out to a depth of $200 \mu \mathrm{m}$ using a $400 \mu \mathrm{m}$ diameter end mill (Drill Service Ltd., www.drill-service.co.uk). The 3 inlet/outlet holes were drilled using a $1.6 \mathrm{~mm}$ drill bit and fitted with polypropylene tubing (cut from EFD dispenser tips, www.efd-inc.com), glued in place. The cell was sealed by gluing (Norland Products Inc. optical adhesive \#61, www.norlandprod.com) a coverslip over the 
channels. Silicone tubing of $1.0 \mathrm{~mm}$ bore (Altec Products Ltd., www.altecweb.com) was used to connect to the $1 \mathrm{ml}$ syringes (Fisher Scientific, www.fisher.co.uk). A KDS200 syringe pump (KD Scientific, www.kdscientific.com) was used to drive the solutions through the flow cell at a controlled rate.

\subsubsection{Coverslip surface treatments}

Effective coating of the glass required very clean coverslips. This was completed before the various coatings were applied by soaking in a $0.1 \mathrm{M} \mathrm{NaOH}$ solution for $15 \mathrm{~min}$ followed by vigorous and repeated rinsing with sterile $\mathrm{dH}_{2} \mathrm{O}$ before air drying (Krylov et al., 2000). Care was taken to only handle the coverslips with tweezers or sterile gloved hands.

Poly-L-lysine was used to coat coverglass when it was desirable to enhance adherence of cells to the glass surface. $1 \mathrm{ml}$ of a $0.01 \%$ solution of poly-L-lysine was used to coat a large coverglass $\left(\sim 30 \mathrm{~cm}^{2}\right)$. The coverslips were then gently rocked for $5 \mathrm{~min}$ to ensure an even coating before thorough rinsing with $\mathrm{dH}_{2} \mathrm{O}$ and air drying.

PVA was used to coat coverglass when it was desirable to prevent the adherence of cells and polystyrene microspheres to the glass surface (Krylov et al., 2000). $20 \mu \mathrm{l}$ of a 10\% w/v solution of PVA in water (dissolved over 2 days by continually shaking, importantly avoiding the production of air bubbles) was distributed in an array of small droplets over the surface of a large coverslip $\left(\sim 30 \mathrm{~cm}^{2}\right)$. A second coverslip was then presses against this to spread the PVA solution between the two pieces of glass. The two coverslips were then separated by sliding them across each other before baking overnight at $140{ }^{\circ} \mathrm{C}$. Finally they were washed with water and air dried (S. Krylov, personal communication). Smaller areas of a single coverglass could be selectively and differentially coated by adapting this technique.

\subsubsection{Preparation of microspheres}

Polystyrene microspheres of $1 \mu \mathrm{m}, 4 \mu \mathrm{m}$ and $10 \mu \mathrm{m}$ diameter were used (Sigma, www.sigmaaldrich.com). The dilutions used for these beads is show in Table 2.14. To prevent adherence to the cells or glass the beads were coated in bovine serum albumin (BSA). A $1 \% \mathrm{w} / \mathrm{v}$ solution of BSA in sterile water was prepared. Beads were then added to this solution for $10 \mathrm{~min}$ before spinning down in a microfuge and washing with Vogel's liquid media once prior to use. This solution was then used as the droplet added to the coverslip in sample preparation. Porous silicon beads (a kind gift from C.G. Hunt, USDA Forest Products Laboratory, Madison, WI, USA) of $1 \mu \mathrm{m}$ diameter were used for the 
localised delivery of latrunculin-B. The beads were soaked in a $50 \mu \mathrm{M}$ solution of latrunculin-B (Calbiochem - Merck Biosciences, www.merckbiosciences.co.uk) before washing once with Vogel's liquid media immediately prior to use.

Table 2.14 Dilution of bead solution for use

\begin{tabular}{ccc}
\hline Microsphere & $\begin{array}{c}\text { Initial dilution in } \\
\text { water for stock }\end{array}$ & $\begin{array}{c}\text { Dilution in Vogel's } \\
\text { liquid media for use }\end{array}$ \\
\hline $1 \mu \mathrm{m}$ & $0.2 \% \mathrm{v} / \mathrm{v}$ & $0.25 \% \mathrm{v} / \mathrm{v}$ \\
$4 \mu \mathrm{m}$ & $2 \% \mathrm{v} / \mathrm{v}$ & $10 \% \mathrm{v} / \mathrm{v}$ \\
$10 \mu \mathrm{m}$ & $2 \% \mathrm{v} / \mathrm{v}$ & $10 \% \mathrm{v} / \mathrm{v}$ \\
\hline
\end{tabular}

\subsubsection{Preparation of dyes for fluorescence microscopy}

Imaging experiments, on the confocal microscope or using widefield fluorescence utilised the inverted agar block method described, with the dye dissolved in the liquid droplet (Table 2.15). Due to the limited solubility in aqueous solutions it was necessary to first dissolve the dye in an organic solvent (DMSO), which was kept below $0.2 \%(w / v)$ in the final solution used.

Table 2.15 Fluorescent dyes used (data from Invitrogen Molecular Probes, www.probes.invitrogen.com).

\begin{tabular}{cccccc}
\hline Dye & Selectivity & $\begin{array}{c}\text { Concentration } \\
(\mu \mathrm{M})\end{array}$ & $\begin{array}{c}\text { Exitation } \\
\text { max. }(\mathbf{n m})\end{array}$ & $\begin{array}{c}\text { Emission } \\
\text { max. }(\mathrm{nm})\end{array}$ & $\begin{array}{c}\text { Molecular } \\
\text { weight }\end{array}$ \\
\hline FM4-64 & Membranes & $<25$ & 514 & 734 & 608 \\
$\begin{array}{c}\text { Oregon } \\
\text { Green 488 }\end{array}$ & Vacuoles & 20 & 488 & 517 & 496 \\
\hline
\end{tabular}

\subsection{Microscopy}

A range of microscopy techniques were used throughout the experiments to enable enhanced visualisation of specific structures or organelles within the cells. For unstained samples phase contrast and differential interference contrast (DIC) microscopy were used (section 2.5.1). Fluorescent dyes in combination with widefield fluorescence, including deconvolution, (section 2.5.2) or confocal laser scanning microscopy (CLSM; section 2.5.3) were used to further enhance the imaging and localisation of specific organelles or cellular processes. Nikon TE2000 inverted microscopes (www.nikon.com) were used throughout, 
excluding the holographic optical tweezer experiments, which was a custom built open bench optical system (section 2.6.3).

\subsubsection{Bright-field, phase contrast and differential interference contrast microscopy}

Standard light microscopy forms an image of an object based on its interaction with the transmission illumination passing through the sample (e.g. absorption, optical path differences, phase gradients and birefringence). Prior to all experiments Koehler illumination was set up appropriately to ensure even illumination and maximal spatial resolution giving the best possible images for each sample. Basic brightfield microscopy was used in all experiments in which force calibration or measurement was completed. This limited the number of optical components the laser had to pass through, and therefore attenuation, to a minimum when image quality was not of the highest importance. A Nikon Plan Fluor 100x 1.4 N.A. DIC H oil immersion or a Nikon Plan Apo $4 \times 0.2$ N.A. objective was used, or those described in section 2.6 .3 when using the holographic optical tweezers. Whilst fungal cells are visible with standard bright-field illumination it was preferable to enhance the contrast in the image using either phase contrast or DIC optics.

Phase shifts are often induced in the light passing through biological samples. Whilst the human eye is unable to detect such differences in light, phase contrast microscopy transforms these sample induced phase differences into differences in amplitude in the final image. Dark objects within the image represent objects with a higher refractive index than their surrounding media and light objects have a lower refractive index (Murphy, 2001). This is particularly useful for optical tweezer experiments as the refractive index, relative to its surroundings, of an object is the main determinant in whether or not that object can be trapped. The combination of a condenser annulus and a matching objective lens containing a phase plate enables phase contrast microscopy on an otherwise normal microscope. In practice a Plan Fluor $100 \times, 1.3$ N.A. Ph 3 DLL oil immersion objective lens was used with the corresponding $\mathrm{Ph} 3$ condenser annulus.

DIC microscopy is a technique in which dual-beam interference optics are used to produce amplitude contrast in an image from local gradients in optical path length (a product of refractive index and thickness of an object through which light is travelling). Again this is useful in optical tweezer experiments as bright objects may well have a sufficiently high refractive index to be trapped (although it could also be a product of the thickness of the object). The images produced are very distinctive for their shadow-cast, 3D-like appearance 
(Murphy, 2001). To implement DIC microscopy, in an inverted microscope, a polarizer and Wollaston prism are mounted in the condenser and complementary Wollaston prism slider and analyser are mounted beneath the objective lens. The sample is then focussed and DIC adjusted using the slider. A Nikon Plan Fluor $100 \times 1.4$ N.A. DIC H oil immersion or a Nikon Plan Apo $60 \times 1.2$ N.A. DIC H water immersion objective lens were used, with the corresponding slider.

\subsubsection{Widefield fluorescence microscopy}

Fluorescence microscopy, in contrast to the techniques so far described, depends upon the presence of fluorescent molecules that when exposed to the appropriate excitation light will emit light of a longer wavelength (Murphy, 2001). The plethora of recombinant fluorescent probes, chemical dyes and filter sets now available permit the visualisation of very specific molecules, organelles and cellular processes which can otherwise not be seen. Excitation light, from a mercury arc lamp, xenon arc lamp or more recently LEDs, illuminates cells through the objective lens using epi-illumination. The same objective lens then functions to collect the emitted fluorescence light as it would in transmitted light microscopy. A filter set, constituting an excitation filter, a dichroic mirror and an emission filter serves to prevent any unwanted wavelengths of light reaching the detection device. High sensitivity CCD cameras (Hamamatsu Orca-ER, www.hamamatsu.com and LaVision ImagerQE, www.lavision.de were used here) are then used to permit the limitation of the exposure to excitation light, which can be damaging to cells. The high contrast images produced have bright areas of fluorescence, corresponding to where the fluorescent probe is concentrated, on a black background. This allows for the selective imaging of cell components and cellular processes of interest. Either a standard Nikon mercury arc lamp (regulating the power with either an $8 \times$ or $4 \times$ neutral density filter, or a combination of the two) or a Till Polychrome IV monochromator xenon arc lamp (www.till-photonics.com) was used with a Nikon Plan Fluor $100 \times 1.4$ N.A. DIC H oil immersion objective. For GFP, FM4-64 and Oregon Green imaging the Nikon B-2A filter cube was used (Table 2.16).

Table 2.16 Details of Nikon B-2A filter cube used for widefield fluorescence microscopy.

\begin{tabular}{cccc}
\hline Filter set & Excitation filter & Dichroic mirror & Emission filter \\
\hline Nikon B-2A & $470 / 20 \mathrm{~nm} \mathrm{BF}$ & $500 \mathrm{~nm} \mathrm{LP}$ & $515 \mathrm{~nm} \mathrm{LP}$ \\
\hline
\end{tabular}

Abbreviations: $\mathrm{BF}=$ Barrier filter, $\mathrm{LP}=$ longpass filter 


\subsubsection{Deconvolution microscopy}

Deconvolution microscopy is a technique of processing image data, obtained on a highly automated widefield fluorescence microscope, to deblur and improve contrast and resolution (Wallace et al., 2001; Parton and Davis, 2005). Information on how the microscope system performs is used to apply an iterative mathematical transformation that results in blur free confocal-like optical sectioning in the captured images. A DeltavisionRT system (Applied Precision, www.api.com) designed specifically for deconvolution microscopy was used here with an Olympus Plan Apo $60 \times 1.2$ N.A. water immersion objective lens.

\subsubsection{Confocal microscopy}

Confocal laser scanning microscopy (CLSM) allows the rejection of fluorescent light emitted from parts of the sample above and below the plane of focus using an aperture in front of the detector, thereby increasing the resolution and contrast within an image (Czymmek et al., 1994; Pawley, 1995; Sheppard and Shotton, 1997; Diaspro, 2001). Confocal microscopy was performed using a Bio-Rad Radiance 2100 (now Zeiss, www.zeiss.com) system equipped with $40 \mathrm{~mW}$ argon ion laser, and mounted on a Nikon TE2000-U microscope. GFP and FM4-64 were imaged after excitation with the $488 \mathrm{~nm}$ laser line set to $\leq 15 \%$ and fluorescence detected at 510-560 $\mathrm{nm}$ and $>600 \mathrm{~nm}$ respectively. A Nikon Plan Apo 60× 1.2 N.A. water immersion or a Plan Fluor $100 \times 1.4$ N.A. DIC H oil immersion objective lens were used.

\subsubsection{Settings for fluorescence imaging}

Settings for fluorescence imaging, both widefield and confocal, are a compromise between image quality (signal to noise ratio) and a need to minimise stress to cells, through phototoxic effects, and dye photobleaching (Pawley, 1995; Hickey et al., 2005). The epiillumination was set up using the apertures on the epifluorescence housing to minimize the area of illumination to just the field of view, thereby preserving cells outside it. When imaging hyphae with the confocal microscope, hyphae were orientated horizontally relative to the frame width allowing reduction of the scan area to, again limit exposure to the cells in the field of view. The length and intensity of exposure was also limited, by shuttering off the light or using higher scan speeds over smaller areas. 


\subsection{Optical tweezer set ups}

There were three main optical tweezer systems used in the work presented. Firstly, one $785 \mathrm{~nm}$ system was designed to be used by biologists lacking an advanced knowledge of optics with the aim of being a simple, compact, easy-to-use, safe and robust and mounted on a research grade inverted light microscope. The tweezer system was continually developed and improved throughout the project to increase its functionality, both within the software and in terms of the hardware (described in sections 2.6.1 and 2.6.2). Secondly, in collaboration with D.R. Burnham and D. McGloin of the University of St. Andrews, a simple open-bench optical tweezer system was used for tweezer trapping with $1064 \mathrm{~nm}$ infrared light (section 2.6.1). Thirdly, also in collaboration with the University of St. Andrews, an open-bench $532 \mathrm{~nm}$ holographic optical tweezer system was developed (section 2.6.3).

\subsubsection{Single-trap optical tweezer systems}

The first version of the optical tweezer set-up was built (by Jochen Arlt) as a single steerable trap system (Fig. 2.4). The main upgrade during the development of the tweezers was to change the camera and microscope capabilities to one more suitable for biological experiments (Fig. 2.5). The system contains a near-infrared $(\lambda=785 \mathrm{~nm})$ diode laser, the output power of which is adjustable up to $70 \mathrm{~mW}$ (VPSL-0785-070-x-5-A; Blue Sky Research, www.blueskyresearch.com). The laser beam is directed first towards two galvanometric beam steering mirrors and then into the rear of the microscope (Figs. 2A and C). A slight modification made to the microscope, which involved lifting the nosepiece, allows the optical tweezers to be combined with fluorescence imaging. A dichroic mirror reflects the beam into the objective lens (Nikon Plan Fluor 100× 1.4 N.A. DIC H oil immersion unless specified). The beam is expanded to fill the back aperture of the objective to offer maximal trapping efficiency. A computer program written in LabVIEW (v. 6.1; National Instruments, www.ni.com) allows the user to move the trap in $x$ and $y$ directions across the field of view by controlling the galvanometric mirrors at the click of the mouse. The speed of trap movement can be preset in $\mu \mathrm{m} \mathrm{s}^{-1}$ in the tweezer control program. The computer takes the images generated by the CCD camera (Applied Vision Technologies, Dolphin 145-F, from FirstSight Vision, www.firstsightvision.co.uk), and moves the trap in a straight line at constant speed to any position in the field of view. A double click releases the trapped object. Movement in the $z$ axis is made by adjusting the focus of the microscope. It is important to note here that the laser power at the specimen is approximately $50 \%$ of the laser output power due to attenuation through the microscope, particularly the objective lens 
(Svoboda and Block, 1994; Neuman et al., 1999; Leitz et al., 2002). In this thesis laser power is referred to in terms of output power.

For experiments featuring low light fluorescence microscopy a second more sensitive camera was also used. A LaVision Imager QE cooled CCD camera (www.lavison.de) was mounted on the microscopes left-port and operated with a second computer running the LaVision DaVis imaging software.

In collaboration with D.R. Burnham and D. McGloin of the University of St. Andrews, a simplified open-bench optical tweezer system was used for single trap experiments with $1064 \mathrm{~nm}$ light as has been described previously (Garces-Chavez et al., 2004).
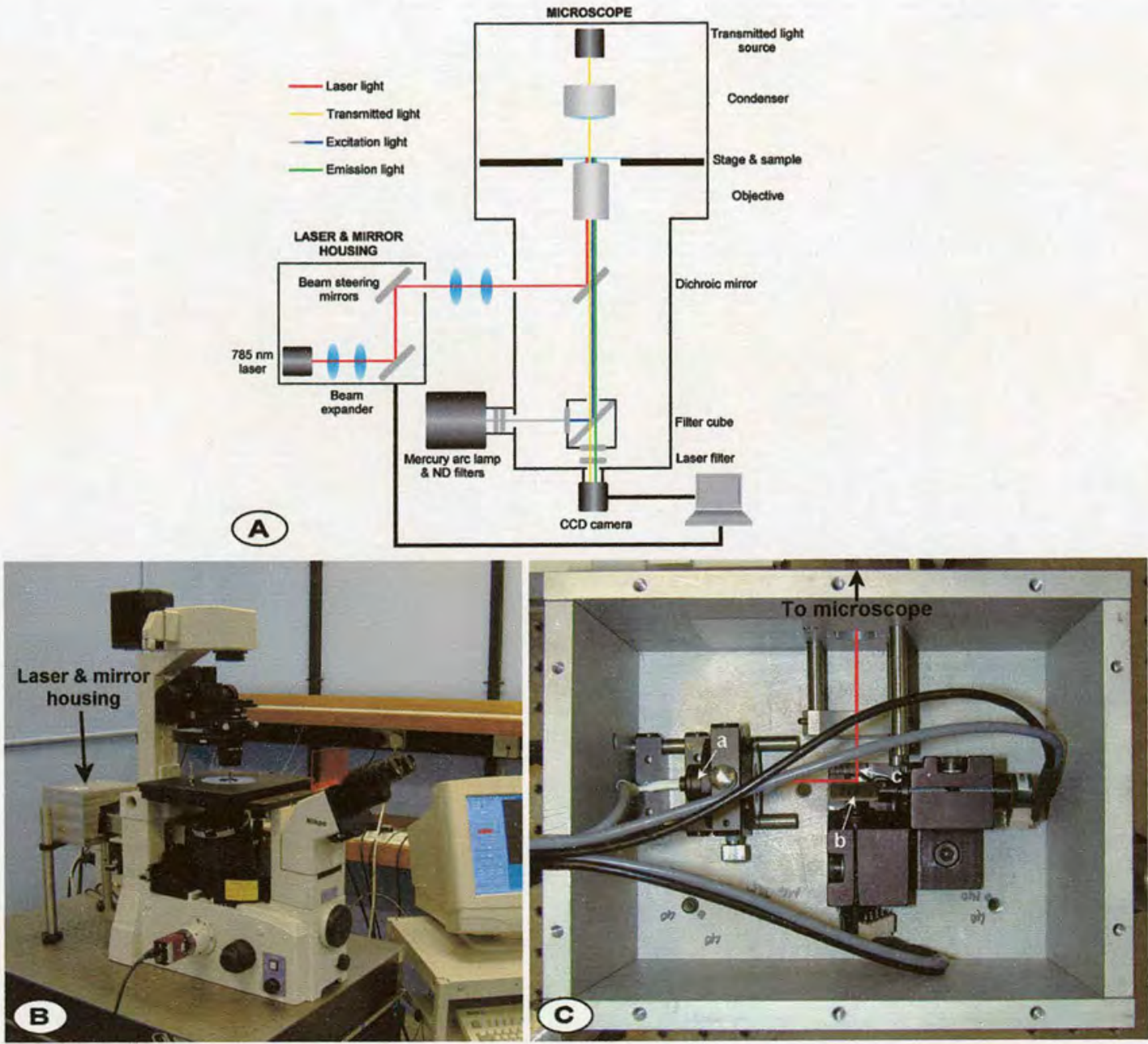

Figure 2.4 (A) Schematic diagram of the optical tweezer system used. (B) The tweezer system mounted on the microscope. (C) The interior of the laser and mirror housing in which (a) is the $785 \mathrm{~nm}$ diode laser, (b) are the galvanometric mirrors, and (c) is the path taken by the laser beam. The optical elements required for phase contrast, DIC and fluorescence can be incorporated into the microscope whilst maintaining the full functionality of the optical tweezers (from Wright et al., 2007). 

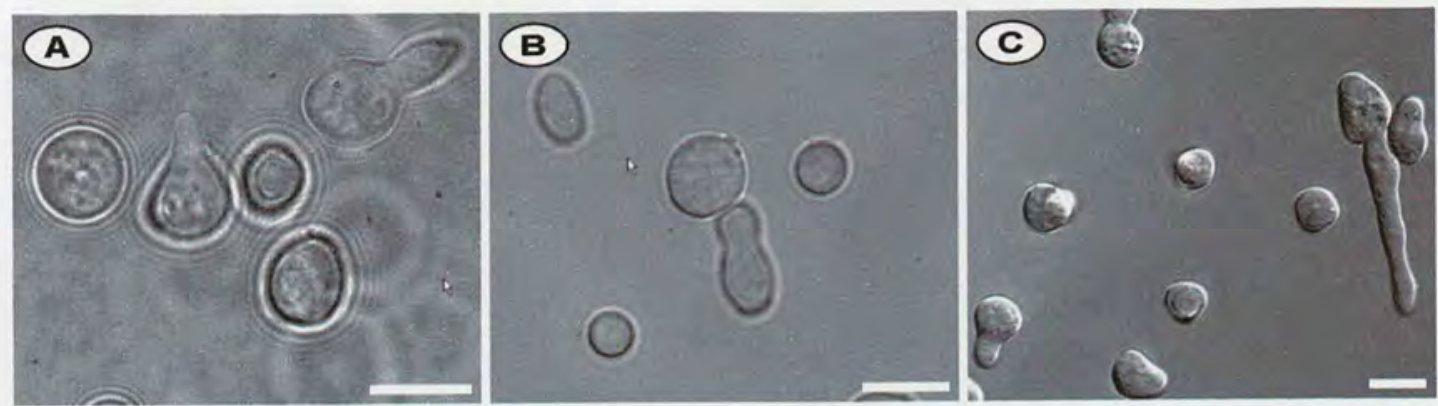

Figure 2.5 Improving the camera and microscope capabilities on the tweezer setup demonstated by images of conidia and conidial germilings of $N$. crassa. (A) The initial setup, using brightfield imaging a basic 'webcam' to capture the image. (B) Brightfield imaging using an improved camera. (C) DIC imaging with the AVT Dolphin 145-F after DIC optics were introduced to the microscope, improving the contrast of the cells and organelles within the cells. A $0.7 \times$ demagnifying C-Mount adapter was also added to increase the field of view. Bars $=10 \mu \mathrm{m}$.

\subsubsection{Dual-trap optical tweezer system}

To add further functionality to the tweezer system a second trap was added into the laser and mirror housing (Fig. 2.6). The same type of laser as the original trap was added to produce a fixed trap at the centre of the field-of-view, otherwise the system remained the same as described in section 2.6.1.

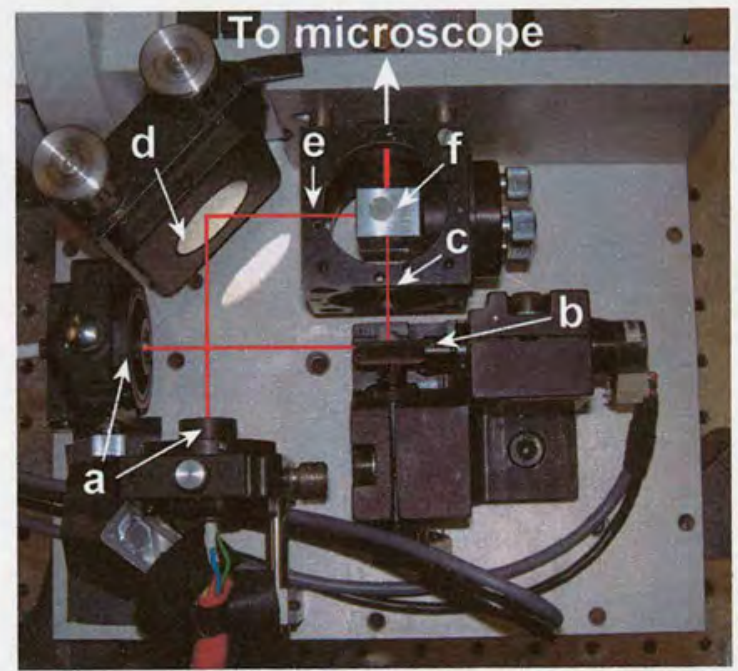

Figure 2.6 The interior of the laser and mirror housing of the dual trap tweezer system in which (a) are the $785 \mathrm{~nm}$ diode lasers, (b) are the galvanometric mirrors, (c) is the path taken by the steerable laser beam, (d) is a fixed mirror, (e) is the path taken by the static laser, and $(f)$ is a polarising beam splitter which combines the beams before they enter the microscope.

\subsubsection{Holographic optical tweezer system}

A schematic of the holographic optical tweezers apparatus is shown in Fig. 2.7 (built in collaboration with D. Burnham and D. McGloin). The diffraction efficiency of a Holoeye 
LC-R 2500 spatial light modulator (SLM; www.holoeye.com) is optimized by rotating a linearly polarized beam from a Laser Quantum Finesse $532 \mathrm{~nm}$ laser (www.laserquantum.com) with a half-wave plate. The beam is then expanded to entirely fill the width of the SLM. Two $4 \mathrm{f}$ imaging systems are designed and positioned such that the SLM is conjugate to the back aperture of either a Nikon Plan or a Nikon E Plan, $100 \times 1.25$ NA oil microscope objective whilst slightly overfilling the aperture. The same objective, in conjunction with custom built Koehler illumination and a $200 \mathrm{~mm}$ tube lens, formed an image on a Basler A602f Firewire CCD camera (www.baslerweb.com).

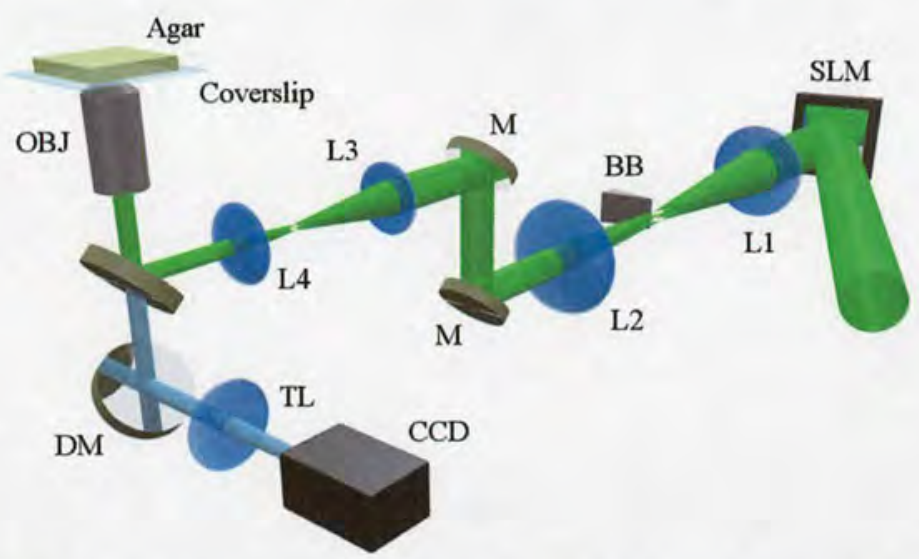

Figure 2.7 Schematic showing the holographic optical tweezers apparatus. Lenses L1 and $\mathrm{L} 2$ and $\mathrm{L} 3$ and L4 form two $4 \mathrm{f}$ imaging systems, placing the objective back aperture conjugate to the SLM. BB is a beam block to remove the unwanted zeroth diffracted order. OBJ is either a Nikon Plan or a Nikon E Plan 100× 1.25 NA oil immersion microscope objective. DM is a dichroic mirror, $M$ are dielectric mirrors and TL is a tube lens. The sample is illuminated with custom built Koehler illumination (not shown) (from Burnham et al., 2007).

\subsubsection{Hologram generation}

To generate the phase-only holograms, or kinoforms, whose optical Fourier transform gives the desired intensity pattern in the objective's focal plane, one of two algorithms was implemented using custom written LabVIEW programs. The first was a superposition of complex functions describing gratings and lenses (Liesener et al., 2000) integrated into an interactive 'point and click' (Leach et al., 2006) program. The second used a slightly modified Gerchberg-Saxton algorithm, the adaptive-additive algorithm (Soifer et al., 1997), which allowed the user to input an arbitrary 8-bit greyscale image containing the desired intensity pattern whether it be single points or continuous lines. It is important to note that SLMs are notorious for having irregularly shaped back planes. When focusing their reflected collimated beams to a diffraction limited spot it becomes obvious that a significant amount of aberration has been induced in addition to that from misalignment in the optical train 
alone. By implementing another custom written LabVIEW program using adaptive optics without a wavefront sensor (Wulff et al., 2006) the aberration could be quantified in terms of Zernike polynomials and coefficients (Love, 1997). This information was used to generate a correction kinoform, simply by adding mod $2 \pi$ to the normal kinoform, that compensated for the system's aberrations and results in a six-fold increase in the Strehl ratio for Gaussian foci. The Strehl ratio increase was calculated from the ratio of the peak intensities after and before correction. The continuous light patterns produced only exist with a reasonable fidelity in a relatively shallow axial (along the beam propagation direction) plane. Experiments with axially extended light fields, or 'pseudowalls' of light, were also performed. These walls consisted of multiple, two dimensional, continuous light patterns displaced above and below the normal focus. Figure 2.8 shows a diagram of the clear difference between these pseudowalls and the simple, axially compact structures normally produced. The kinoform to be placed on the SLM is created simply by calculating those required to produce particular patterns at desired axial planes and then finding the argument of their complex sum. The result is a single, phase-only hologram producing patterns in multiple, spatially separated, axial planes.
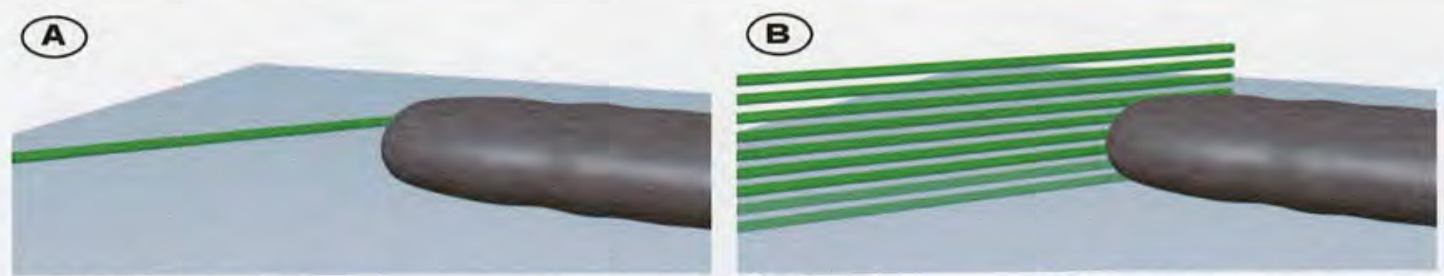

Figure 2.8 Diagrammatical representation of a hyphal tip approaching (A) a single line of light, and (B) nine, axially stacked, single lines of light, or a 'pseudowall' (from Burnham et al., 2007).

\subsubsection{Force calibration for optical tweezers}

To calibrate the trapping force produced by the tweezers the escape force method (Greulich, 1999) was used. This method involved trapping a bead and, using a motorised stage (custom built from Coherent parts, www.coherent.com), moving its environment past it at increasing velocity until the bead escapes the trap, then applying Stoke's law (Chapter 5). The trap speed was controlled using a custom written LabView programme.

\subsection{Digital image processing and animations}

Images from the optical tweezers were captured either singly through the tweezers LabView control software directly from the camera or as a time-course using screen capture software 
(Hypercam, www.hyperionics.com). Further processing was carried out with Paintshop Pro (v. 7 \& v. 8; JASC Software, now Corel, www.corel.com), ImageJ (freeware; http://rsb.info.nih.gov/ij/), SimplePCI (Compix Inc. Imaging Systems, www.cimaging.net) and custom written LabView programmes. Time-courses of images were edited and built up into animation movies (.avi and .mpg files) using Animation Shop (v. 3; JASC Software, now Corel, www.corel.com), Premier Pro (v. 1.5; Adobe, www.adobe.com), Windows Movie Maker (v. 1.5; Microsoft, www.microsoft.com), and ImageJ (freeware; http://rsb.info.nih.gov/ij/).

Confocal images were captured using Lasersharp software (v. 5.2 and v. 6.0; Bio-Rad, now Zeiss, www.zeiss.co.uk) and were initially viewed using Confocal Assistant software (v. 4.02), before further processing as above.

\subsection{References}

Burnham, D.R., Wright, G.D., Read, N.D., McGloin, D., 2007. Holographic and single beam optical manipulation of hyphal tip growth in filamentous fungi. J. Opt. A: Pure Appl. Opt. 9, S172-S179.

Czymmek, K.J., Whallon, J.H., Klomparens, K.L., 1994. Confocal microscopy in mycological research. Exp. Mycol. 18, 275-293.

Davis, R.H., de Serres, F.J., 1970. Genetic and microbiological research techniques for $N$. crassa. In: Tabor, H., White Tabor, C. (Eds.), Methods in Enzymology, Vol. 17. Metabolism of amino acids and amides Academic Press, New York, pp. 79-143.

Diaspro, A., 2001. Confocal and Two-Photon Microscopy: Foundations, Applications and Advances. Wiley-Liss, New York.

Garces-Chavez, V., McGloin, D., Summers, M.D., Fernandez-Nieves, A., Spalding, G.C., Cristobal, G., Dholakia, K., 2004. The reconstruction of optical angular momentum after distortion in amplitude, phase and polarization. J. Opt. A: Pure Appl. Opt. 6, S235-S238.

Greulich, K.O., 1999. Micromanipulation by light in biology and medicine: The laser micobeam and optical tweezers. Birkhauser Verlag, Basel, Switzerland.

Hickey, P.C., Swift, S.R., Roca, M.G., Read, N.D., 2005. Live-cell imaging of filamentous fungi using vital fluorescent dyes and confocal microscopy. Meth. Microbiol. 34, 63-87.

Krylov, S.N., Dovichi, N.J., 2000. Single-cell analysis using capillary electrophoresis: influence of surface support properties on cell injection into the capillary. Electrophoresis 21, 767-773.

Leach, J., Wulff, K., Sinclair, G., Jordan, P., Courtial, J., Thomson, L., Gibson, G., Karunwi, K., Cooper, J., Laczik, Z.J., Padgett, M., 2006. Interactive approach to optical tweezers control. Appl. Opt. 45, 897-903.

Leitz, G., Fallman, E., Tuck, S., Axner, O., 2002. Stress response in Caenorhabditis elegans caused by optical tweezers: Wavelength, power, and time dependence. Biophys. J. 82, 2224-2231. 
Liesener, J., Reicherter, M., Haist, T., Tiziani, H.J., 2000. Multi-functional optical tweezers using computer generated holograms. Opt. Commun. 185, 77.

Lopez-Franco, R., Bracker, C.E., 1996. Diversity and dynamics of the Spitzenkorper in growing hyphal tips of higher fungi. Protoplasma 195, 90-111.

Love, G.D., 1997. Wave-front correction and production of Zernike modes with a liquid-crystal spatial light modulator. Appl. Opt. 36, 1517-1524.

Murphy, D.B., 2001. Fundamentals of Light Microscopy and Electronic Imaging. Wiley-Liss, New York.

Neuman, K.C., Chadd, E.H., Liou, G.F., Bergman, K., Block, S.M., 1999. Characterization of photodamage to Escherichia coli in optical traps. Biophys. J. 77, 2856-2863.

Parton, R.M., Davis, I., 2005. Lifting the fog: image restoration by deconvolution. In: Celis, J.E. (Ed.), Cell Biology, A Laboratory Handbook. Academic Press, London, pp. 187-200.

Pawley, J.B., 1995. Fundamental limits in confocal microscopy. In: Pawley, J.B. (Ed.), Handbook of Biological Confocal Microscopy Plenum Press, New York, pp. 19-37.

Sheppard, C.J.R., Shotton, D.M., 1997. Confocal Laser Scanning Microscopy. IOS Scientific Publishers, Oxford.

Smith, D., Oinons, A.H.S., 1983. A comparison of some preservation techniques for fungi. Trans. British Mycol. Soc. 81, 535-540.

Soifer, V.A., Kotlyar, A., Doskolovich, L., 1997. Iterative Methods for Diffractive Optical Elements. Taylor \& Francis, London.

Svoboda, K., Block, S.M., 1994. Biological applications of optical forces. Annu. Rev. Biophys. Biomol. Struct. 23, 247-285.

Wallace, W., Schaefer, L.H., Swedlow, J.R., 2001. A workingperson's guide to deconvolution in light microscopy. Biotechniques 31, 1076-8, 1080, 1082.

Wright, G.D., Arlt, J., Poon, W.C., Read, N.D., 2007. Optical tweezer micromanipulation of filamentous fungi. Fungal. Genet. Biol. 44, 1-13.

Wulff, K.D., Cole, D.G., Clark, R.L., DiLeonardo, R., Leach, J., Cooper, J., Gibson, G., Padgett, M.J., 2006. Aberration correction in holographic optical tweezers. Opt. Exp. 14, 4169-417 


\section{Chapter 3 - Manipulating whole fungal cells with optical tweezers}

\subsection{Introduction}

The ability to trap whole cells and reposition them with respect to their environment is a technique that offers many opportunities for novel experimentation into the communication of fungal cells with their environment, and particularly into cell-cell communication. The use of optical tweezers has many distinct advantages over more mechanical micromanipulation techniques, namely: 1) it is much less invasive as no mechanical contact is made that could perturb the cells, 2) it allows very precise control over the position of the object being manipulated, 3) trapped objects can be instantly released, and 4) quantitation of the forces applied are possible in the right circumstances (Wright et al., 1990; Berns et al., 1991; Kuo and Sheetz, 1992; Weber and Greulich, 1992; Svoboda and Block, 1994; Sheetz, 1998). Although trapping of a variety of cell types has been demonstrated in the past, a comprehensive study on what can be achieved, and the limitations of using $N$. crassa conidia and germlings, has not been provided. This chapter aims to address that deficit, making a broad assessment of the potential for optical tweezers for manipulating whole cells and developing techniques to refine particular experimental approaches. The recent discovery of conidial anastomosis tubes (CATs) in $N$. crassa has provided a great experimental system in which optical tweezers can be effectively used for experimental micromanipulation (see section 1.2.1.1) (Roca et al., 2005a).

The aims of the experimental research described in this chapter were:

- To show how conidia and conidial germlings can be moved in a controlled manner using optical tweezers

- To improve the control of cell positioning with the introduction of a second independent optical trap

- To further develop the CAT homing assay technique established by Roca et al. (2005a)

- To develop a technique to produce stable micro-arrays of cells for further studies of cellcell communication

- To combine microfluidic flow cells with optical tweezers in order to selectively treat single cells in a cell population (e.g. with a dye or drug) 
- To assess the damage caused to optically trapped cells

Some of the results described in this chapter have been published in Wright et al. (2007a, b).

\subsection{Results}

\subsubsection{Repositioning and re-orientation of conidia}

Ungerminated macroconidia (Fig. 3.1; Mov. 3.1) and germinated macroconidia (Fig. 3.2; Mov. 3.2) were trapped and moved around relative to their surroundings using the initial single trap optical tweezer setup (section 2.6.1). The semi-spherical, ungerminated macroconidia were easily moved within the field of view in $x$ and $y$ directions with the computer-controlled steerable trap. Movement in the $z$ direction was achieved by adjusting the focus position of the microscope. During this process care had to be taken to avoid driving the cell into the coverglass and, in the process, risk losing it from the trap or encourage its adherence to the coverglass surface. Also important was the fact that the trapping efficiency decreased when trapping an object deeper in the sample. Macroconidia could be moved over long distances by trapping them in a fixed position and then carefully, and slowly, moving the microscope stage. Using a moderate laser output power of $40 \mathrm{~mW}$ (from a maximum $70 \mathrm{~mW}$ ) it was possible to move a macroconidium, through liquid Vogel's medium, at speeds of up to $15 \mu \mathrm{m} \mathrm{s}^{-1}$ in $x$ and $y$ directions, without risking losing it from the trap. The speed of movement possible varied depending on the size and optical properties of a given conidium, the laser power used, the viscosity of the medium, and the depth of the conidium in the sample. As a guide, conidia could be trapped and held in the liquid growth medium at a minimum laser power of $5 \mathrm{~mW}$ but the speed of movement was restricted to $<5 \mu \mathrm{m} \mathrm{s}^{-1}$. Using $70 \mathrm{~mW}$ of laser power allowed conidia to be moved at speeds in excess of $40 \mu \mathrm{m} \mathrm{s}^{-1}$.

With time, conidia tended to adhere to the coverglass, after which they could not be detached from the glass surface even when using the maximum laser output power. To overcome this problem the coverslips were coated with PVA (see section 2.4.5), which prevented spore adhesion to the glass without perturbing germ tube growth.

Germ tubes of up to $100 \mu \mathrm{m}$ in length could be trapped at different positions along their length, thereby allowing control of their orientation (Fig. 3.2; Mov. 3.2). When trapping conidia with short germ tubes, care had to be taken to prevent the long axis of the germinated spore from aligning with the direction of beam propagation (i.e. standing vertically within the sample). Reducing the laser power to the minimum necessary prevented this from 
happening. After the addition of the second trapping laser (section 2.6.2) a germling could be trapped by both lasers simultaneously offering much greater control over the cells position and orientation (Fig. 3.3; Mov. 3.3).

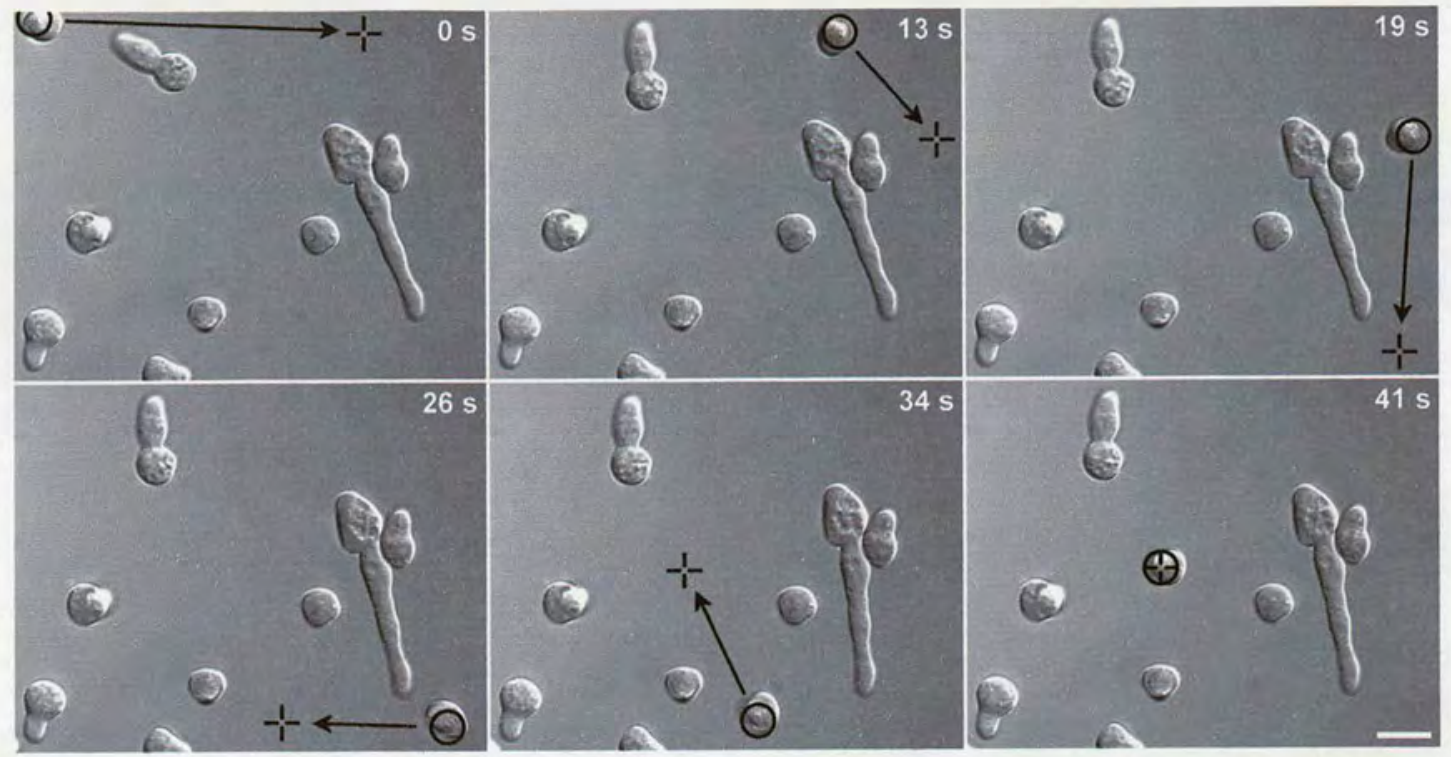

Figure 3.1 Micromanipulation of an ungerminated macroconidium of $N$. crassa. The circle represents the current position of the laser whilst the cross-hair shows the position to which the trap is about to be moved. DIC microscopy, $100 \times$ objective. Bar $=10 \mu \mathrm{m}$. (see Mov. 3.1) (from Wright et al., 2007a)

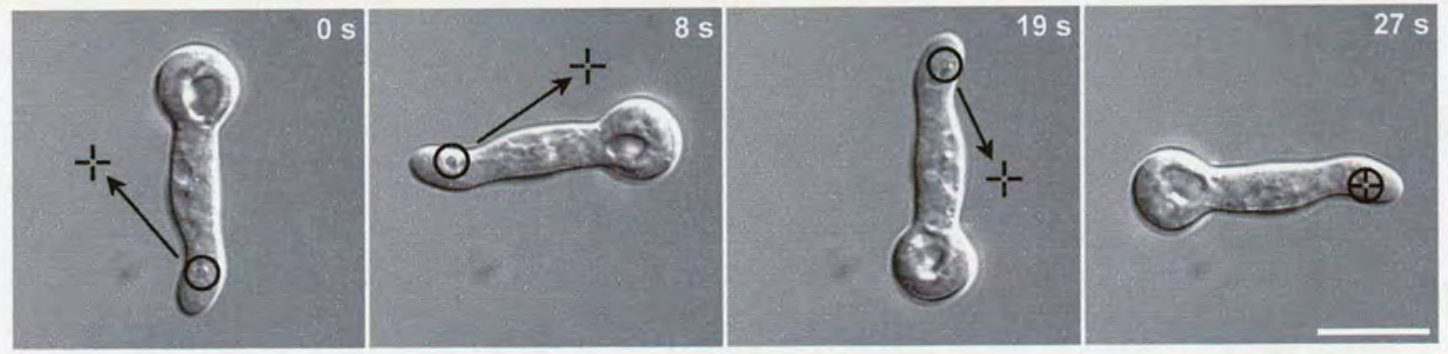

Figure 3.2 Rotation of a macroconidial germling of $N$. crassa. The circle represents the current position of the laser whilst the cross-hair shows the position to which the trap is about to be moved. Note that as the germling was manipulated it rotated around its centre point, and the field of view remained constant throughout the time course. DIC microscopy, 100x objective. Bar = $10 \mu \mathrm{m}$. (see Mov. 3.2) (from Wright et al., 2007a) 


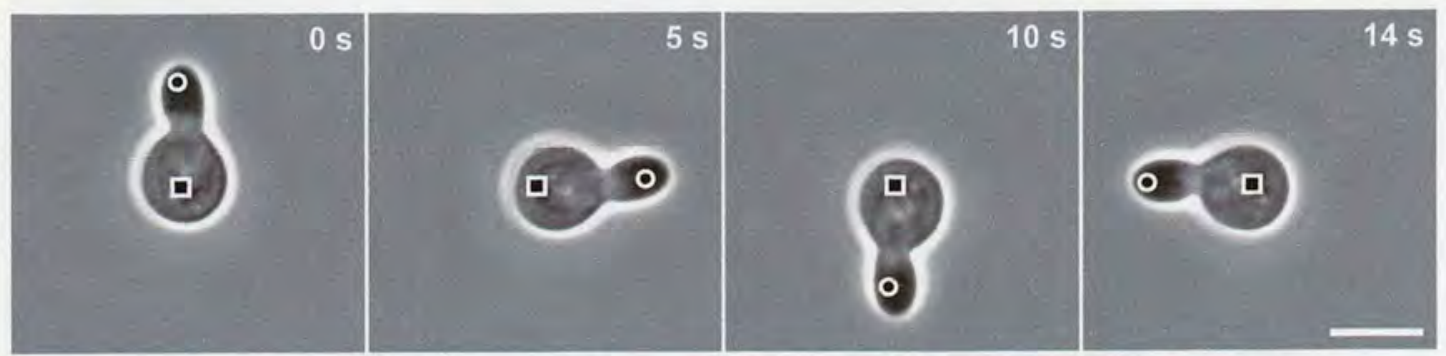

Figure 3.3 Rotation of a macroconidial germling of $N$. crassa using 2 optical traps. The conidium is held by the stationary trap (square); the germ tube tip is held by the moveable trap (circle). In this sequence the germling rotated around the position of the fixed trap and the field of view remained constant throughout the time course. Phase contrast microscopy, $100 \times$ objective. Bar $=5 \mu \mathrm{m}$. (see Mov. 3.3) (from Wright et al., 2007a)

\subsubsection{CAT homing assays}

Optical tweezers have been used to trap and move one CAT (see section 1.4.1) relative to another to unambiguously determine whether the two CATs are homing towards each other or not. This technique has been used to study the signalling pathways involved in CAT homing, particularly by using mutants (Fleißner et al., 2005; Roca et al., 2005a; Roca et al., $2005 \mathrm{~b}$ ). In order to conduct this assay, conidia or germlings were previously only trapped for up to $1 \mathrm{~min}$. Here we found that we could extend the trapping time to $25 \mathrm{~min}$ and found that CATs continued to home towards and fuse with each other, without any apparent ill effects (Fig. 3.4). The development of the PVA technique made these CAT homing assays much more routine, by preventing the adherence of cells to the coverglass surface. Previously, it was up to chance as to whether or not a homing CAT pair could be repositioned with the tweezers because one, or both, partner germlings had commonly adhered to the underlying coverglass. Conidia were inoculated into a multi-well slide and then incubated for 1-3 $\mathrm{h}$ before beginning a CAT homing assay. During this period of time many conidia would adhere to the glass, if it was not coated with PVA. Germ tubes and CAT tips were also found to be particularly sticky. 


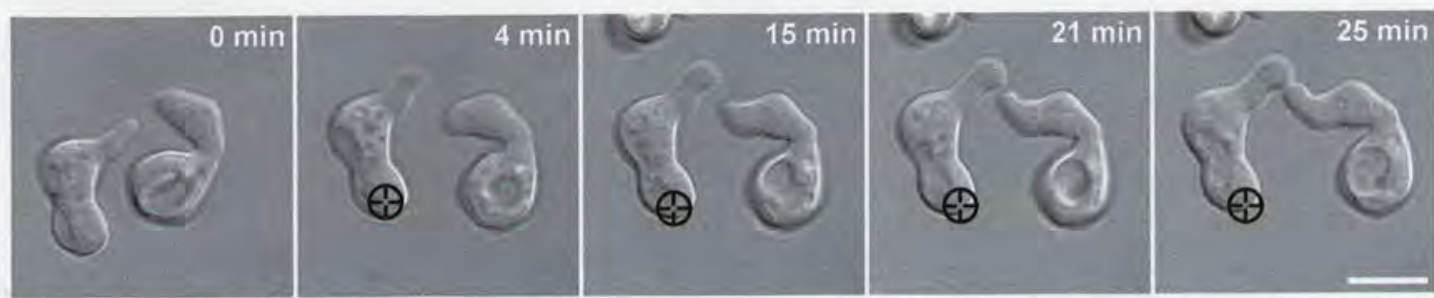

Figure 3.4 An example of a conidial anastomosis tube (CAT) homing assay. The two CATs were homing towards each other $(0 \mathrm{~min})$. The left hand germling was repositioned (here shown $4 \mathrm{~min}$ after repositioning). The CAT tips then changed their direction of growth to home back towards each other (15 and $21 \mathrm{~min}$ ) before making contact $(25 \mathrm{~min})$ and subsequently fusing (not shown). The left hand conidium remained trapped throughout the entire 25 min period without apparent inhibition of CAT growth, homing or fusion. The position of the trap is represented by the cross-hair in the circle. DIC microscopy, 100x objective. Laser power $=10 \mathrm{~mW}$. Bar $=10 \mu \mathrm{m}$. (from Wright et al., 2007a)

\subsubsection{MAP Kinase oscillatory signalling during CAT homing}

Oscillatory recruitment of the MAP kinase protein MAK-2 to CAT tips was recently observed during CAT homing (Roca, Fleißner, Wright, Glass \& Read, unpublished results). An example of this is shown in Fig. 3.5 (Mov. 3.4) in which MAK-2-GFP has been imaged in a $3 \mathrm{D}$ projection with time (i.e. $4 \mathrm{D}$ imaging) using deconvolution microscopy (section 2.5.3). The oscillations in the CAT tips are more clearly seen by quantifying the average fluorescence intensity in boxes placed over the homing CAT tips (Fig. 3.6).

This system seemed to be excellent for using optical tweezers to manipulate the homing CAT tips relative to each other in attempts to experimentally perturb or alter the oscillatory recruitment of MAK-2-GFP. Experiments were designed to manipulate the cells in order to: 1) separate two homing tips undergoing oscillations in MAK-2-GFP recruitment to determine whether two CATs are required in order to produce the oscillations, and the maximum distance between CATs over which the oscillations are maintained, 2) bring together two non-oscillating cells to determine if the MAK-2-GFP oscillations can be induced by the presence of another CAT tip, and 3) re-orientate the relative position of the CAT tips to determine if the localisation of the MAK-2-GFP changes in a reciprocal manner, which would demonstrate continued sensing of the direction of the chemoattractant emitting partner CAT. Unfortunately numerous attempts to perform these optical tweezer experiments on CATs exhibiting these MAK-2-GFP oscillations were unsuccessful, primarily due to excessive photobleaching of the MAK-2-GFP resulting from a lack of control of the excitation illumination. 


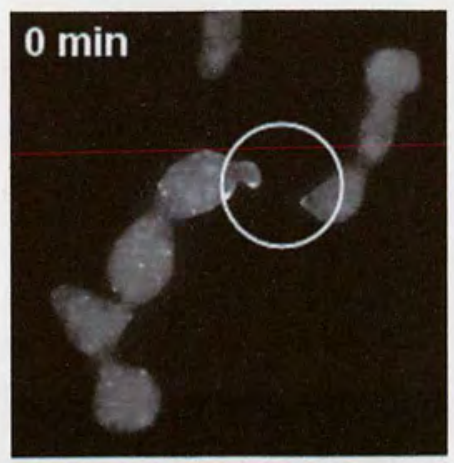

Figure 3.5 Oscillatory recruitment of MAK-2-GFP to CAT tips (within the circle) during homing. 4D image data was collected on a DeltavisionRT system, deconvolved and then each 3D stack projected into a single image. The recruitment of the MAK-2 to the CAT tips is seen to oscillate between the two CAT tips with a period of 5-6 min. Deconvolution widefield fluorescence microscopy, $60 \times$ objective Bar $=10 \mu \mathrm{m}$. (see Fig. 3.6 and Mov. 3.4)
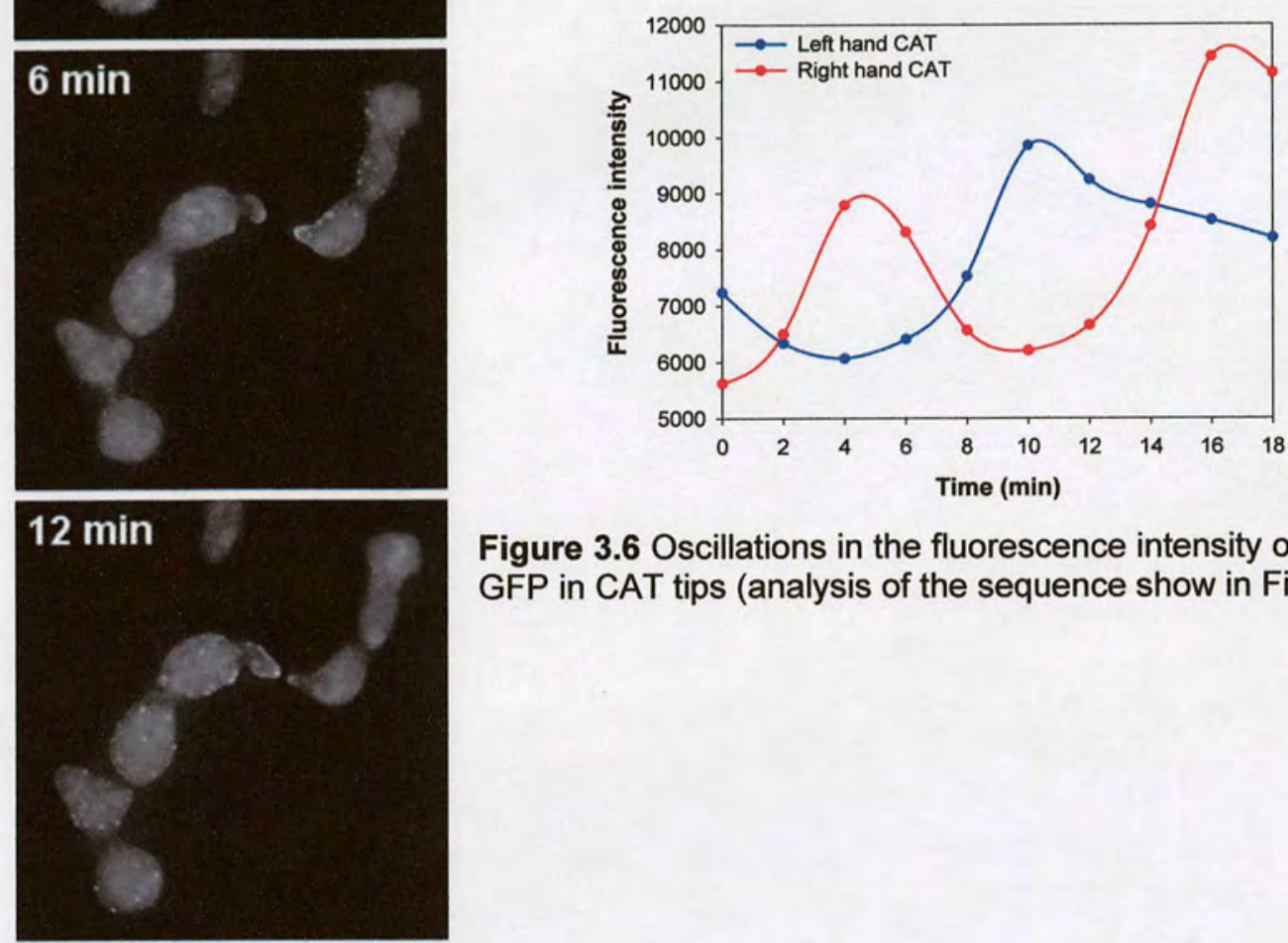

Figure 3.6 Oscillations in the fluorescence intensity of MAK-2GFP in CAT tips (analysis of the sequence show in Fig. 3.5).
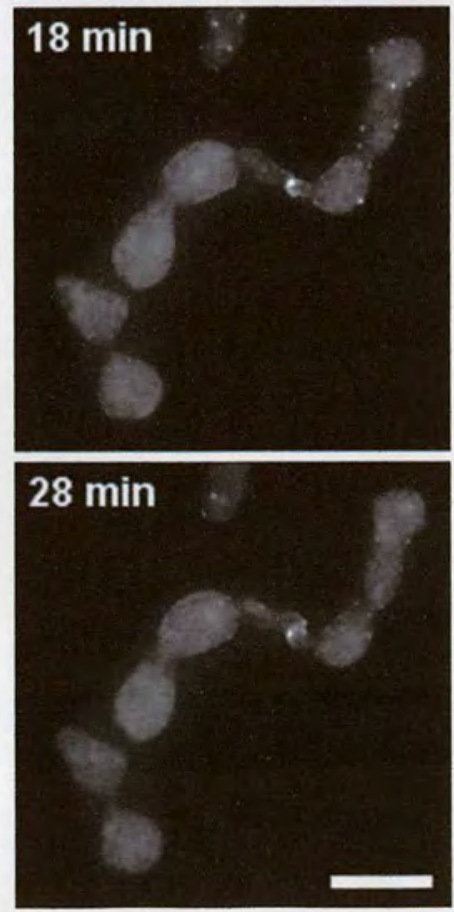


\subsubsection{Micro-arrays of conidia}

Two techniques to establish stable micro-arrays of spores using optical tweezers were developed, one in which conidia were selectively forced to adhere to the surface of a coverglass and a second in which the conidia were held in place in a thermoreversible gel. These techniques provide further experimental approaches to analyse communication between cells within a population

Firstly, by coating half the area of the upper side of a large coverslip with poly (vinyl) alcohol (PVA) a non-stick region of coverglass was created (see section 2.4.5) (Krylov and Dovichi, 2000). Onto the coverglass two wells (rubber grommets with a section cut out) were glued, one on the untreated surface and the other on the PVA treated surface. The gap between the two wells was bridged with a piece of coverglass, cut with a diamond knife, supported by electrician's insulation tape (Fig. 3.7). Ungerminated conidia were then trapped with the optical tweezers and carried over a centimetre across the slide from the PVA coated region into the well above the uncoated surface. Briefly holding the conidia against the glass caused them to adhere. Conidia harvested from older colonies ( $>10$ days) were found to be more adherent than those from 7 day old colonies, whilst still maintaining normal viability. Repeating this procedure allowed fixed arrays of spores to be generated in which the distance separating cells could be accurately defined (Fig 3.8).

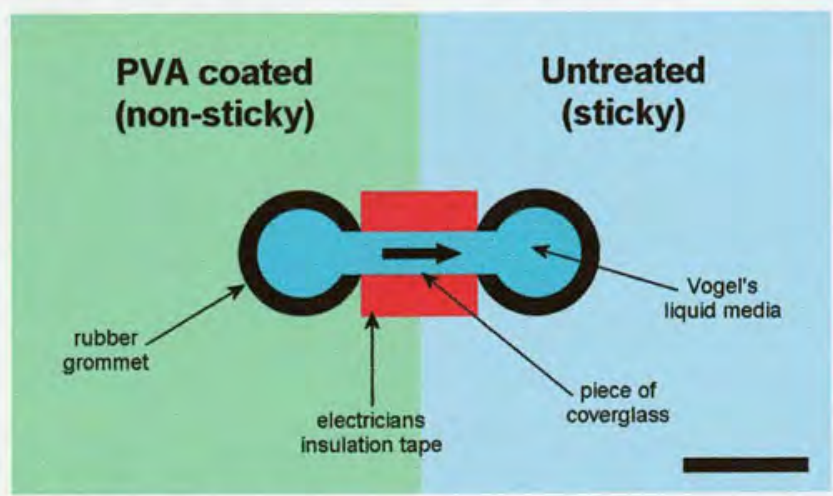

Figure 3.7 Diagram of the PVA slide used for creating micro-arrays of conidia. A PVA coating was applied to half of a large coverslip creating a non-sticky area of glass. Two wells (rubber grommets) were glued and linked by a channel over the boundary between coated and uncoated glass, consisting of a piece of coverglass (cut with a diamond knife) supported by electrician's insulation tape. Conidia were then added to the PVA side and the rest of the chamber filled with liquid Vogel's media. Trapped conidia were then transported through the channel to the untreated side, using the microscope stage controls, where they could be stuck to the glass to form stable micro-arrays (Fig. 3.8). Bar $=1 \mathrm{~cm}$. 


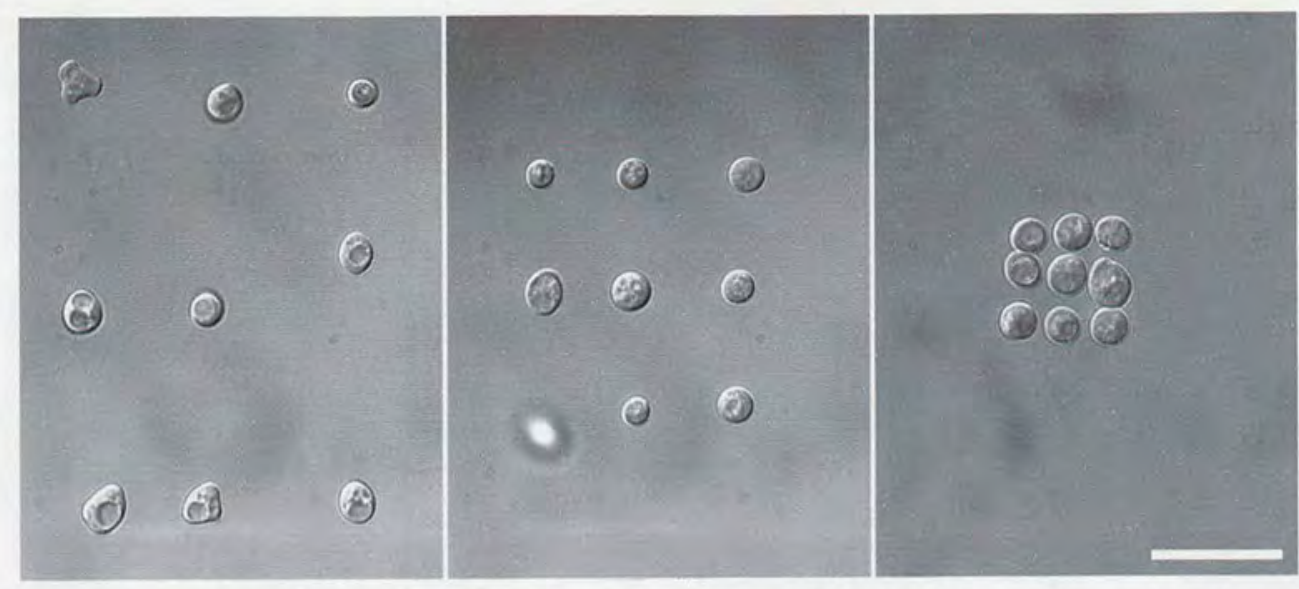

Figure 3.8 Macroconidia were carried from the non-stick PVA side and brought into contact with the uncoated glass, by adjusting the microscopes focus, to cause the macroconidia to adhere. These spore arrays were stable and could be observed over long periods of time whilst germination took place. Three micro-arrays, with varying distances between the conidia are shown. DIC microscopy, $100 \times$ objective. Bar $=25 \mu \mathrm{m}$. (from Wright et al., 2007b)

The second technique developed involved the use of $\mathrm{Cygel}^{\mathrm{TM}}$ (see section 2.4.3). Cygel is a thermoreversible gel that is liquid when cool and gels when warmed; cells will happily grow in the gelled medium. A spore suspension was used to dilute Cygel then, working at room temperature $\left(22{ }^{\circ} \mathrm{C}\right.$, at which the Cygel concentration used was a viscous liquid), a microarray of conidia was brought together and adjusted such that the conidia were distributed as desired. Heating of the sample (to $27^{\circ} \mathrm{C}$ ) then caused the Cygel to set, resulting in the conidia being immobilized such that they could not be moved with the optical tweezers. By allowing the sample to cool back to room temperature the micro-array could then be adjusted once again (Fig. 3.9; Mov. 3.5).

Conidia manipulated in these ways subsequently germinated (data not shown). 


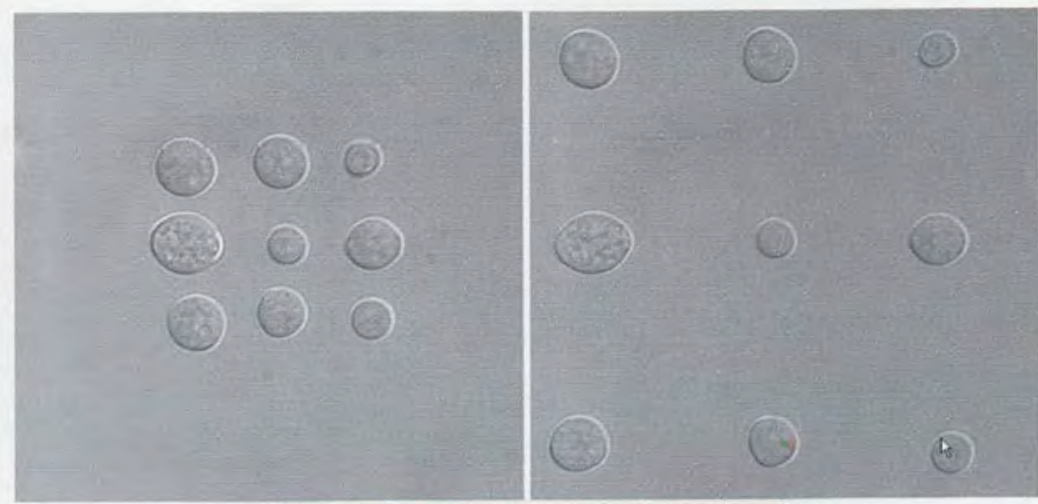

Stage heated to gel media,

stabilising the micro-array.

After 10 mins sample

was cooled.

The gel liquified and

cells could be moved again
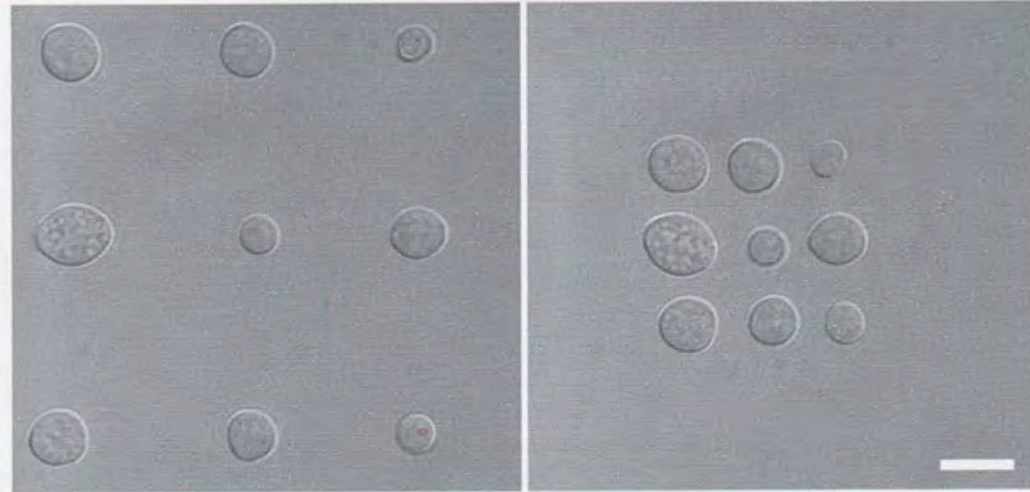

Figure 3.9 Macroconidia were optically trapped and brought together at room temperature $\left(22{ }^{\circ} \mathrm{C}\right)$ in a solution of Vogel's liquid media and Cygel, and then adjusted into a spread out microarray. The stage was then gently heated (taking the sample up to $27^{\circ} \mathrm{C}$ ) causing the medium to gel. The micro-array was stable during the $10 \mathrm{~min}$ period when the medium was gelled and conidia could not be moved with the optical tweezers. The sample was then allowed to cool and cells could once again be moved. DIC microscopy, 100x objective. Bar $=10 \mu \mathrm{m}$. (see Mov. 3.5)

\subsubsection{Microfluidic cells}

The microfluidic cell constructed (see section 2.4.4; Fig. 3.10) produces laminar flow ${ }^{1}$ of two solutions. This creates a sharp boundary between two different solutions, across which the only mixing results from diffusion. As a visual example of how efficient this technique is a blue dye (Supercook food colouring; www.supercook.co.uk) was added to the upper channel

\footnotetext{
${ }^{1}$ Laminar flow: Non-turbulent flow of viscous fluids in parallel layers
} 
and water to the lower (Fig. 3.10). By combining the technique with optical tweezers, cells can be trapped and quickly transferred between the different solutions. In the example shown (Fig. 3.11) a suspension of conidia expressing H1-GFP (nuclear localised GFP) were flowing through the lower channel and a solution of the membrane selective dye FM4-64 (see section 2.4.7) was flowing through the upper channel. A single conidium (exhibiting only GFP fluorescence, $0 \mathrm{~min}$ ) was then trapped with $70 \mathrm{~mW}$ laser power (high power was required to retain the cell in the fast flowing solution) and carried across to the side in which FM4-64 was flowing, the plasma membrane was immediately stained with FM4-64 (Fig. 3.11, $1 \mathrm{~min}$ ). When carried back into the conidial flow it is clear that other conidia lacked the FM4-64 staining (Fig. 3.11, 4 min).

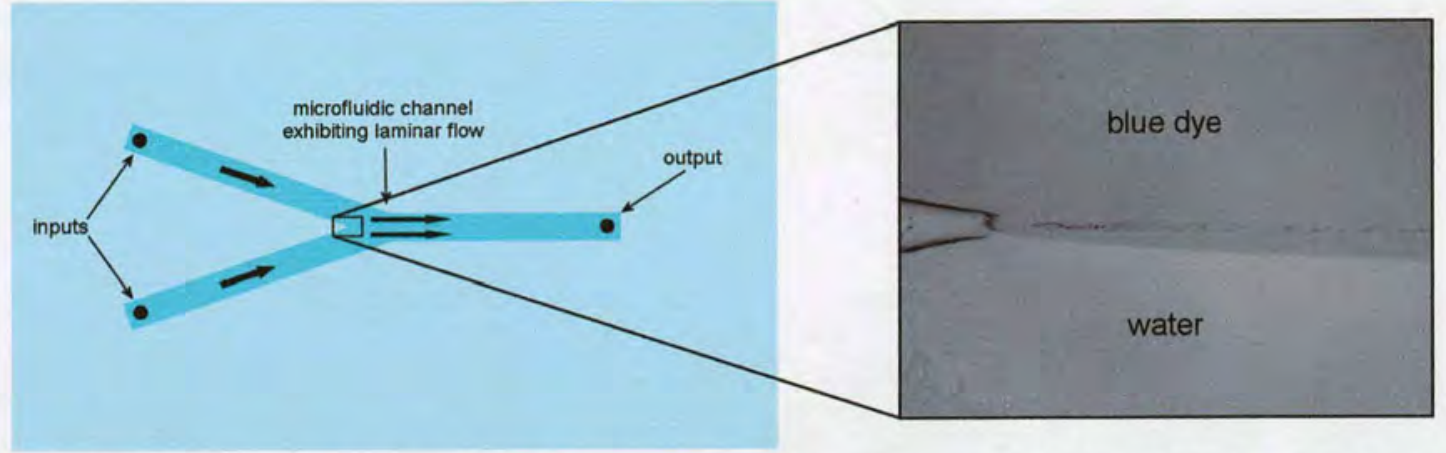

Figure 3.10 Diagram and image of the microfluidic flow cell. Two solutions are driven through the chamber using a syringe pump attached to the two inputs by tubing. Laminar flow results when the two channels merge until the two mix as they exit from the output. On the right an image captured through a Nikon Plan Apo $4 \times 0.2$ N.A objective, to demonstrate the laminar flow of the two solutions is shown.
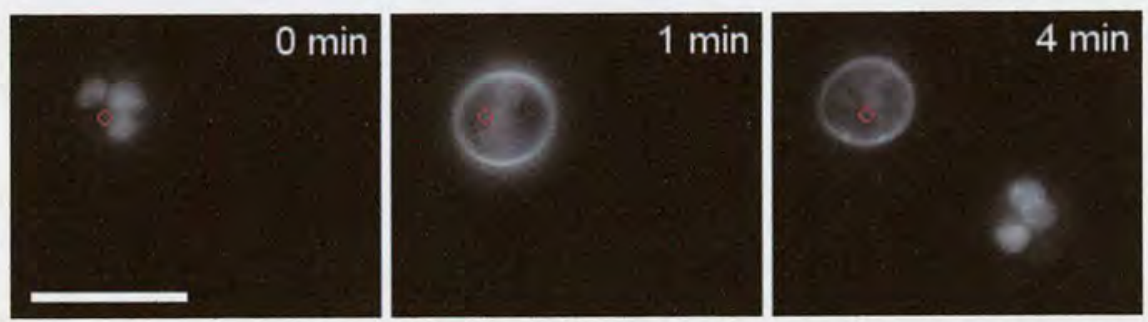

Figure 3.11 A conidium expressing H1-GFP was optically trapped (with $70 \mathrm{~mW}$ laser power) in the lower channel of the microfluidic cell and carried into a solution of the membrane selective dye FM4-64 in the upper channel. 0 min - nuclear labelling in the lower channel, 1 min - nuclear labelling and plasma membrane staining in the upper channel, $4 \mathrm{~min}$ - the conidium shown at the 1 min time point has been moved back into the lower channel and can be seen next to a conidium only exhibiting nuclear labelling. The red circle indicates the position of the laser. Due to photo-bleaching the nuclei of the original cell are successively less bright in the 1 and 4 min images. Fluorescence microscopy, filter cube B-2A, 100x objective. Bar $=10 \mu \mathrm{m}$. 


\subsubsection{Assessment of damage to cells}

\subsubsection{Short term trapping of ungerminated conidia}

A spore germination assay to assess possible damage caused to cells by the trapping laser was performed. This involved analysing the germination of a population of macroconidia in which 50 spores were individually exposed to the trapping laser $(70 \mathrm{~mW})$ for $30 \mathrm{sec}$, the time typically taken to establish a CAT homing assay (Roca et al., 2005a). Their rate of germination was compared to that in a non-irradiated population of macroconidia as a control (Fig. 3.12). No significant difference in the timing of the onset of germination, the rate of germination or final percentage of germinated spores was found.

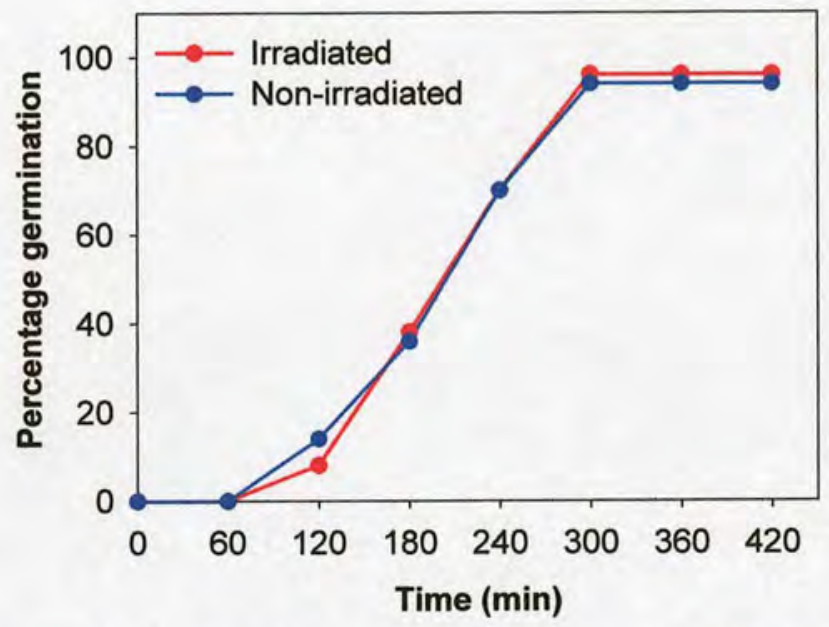

Figure 3.12 Macroconidial germination was not significantly affected in conidia optically trapped compared with those which were not. 50 freshly inoculated ungerminated spores were each trapped for $30 \mathrm{~s}$ and their germination followed for $7 \mathrm{~h}$. Similar results were obtained when this experiment was repeated three times. (from Wright et al., 2007a)

\subsubsection{Long term trapping}

It was shown earlier (Fig 3.4) that a germinated conidium could be trapped continuously (at $10 \mathrm{~mW}$ ) for $25 \mathrm{~min}$ whilst CAT homing and fusion with another CAT continued unimpeded. However, conidia (lacking germ tubes or CATs) trapped for much longer periods of time $(>10 \mathrm{~h}$ ), during which time germination would normally have take place, were unable to germinate even at the lowest useful laser power $(5 \mathrm{~mW})$. On removal of the laser, conidia were unable to germinate even when left overnight (data not shown). Nevertheless, these conidia continued to exhibit normal-looking cytoplasmic and organelle movements indicating that they were still alive (Fig. 3.13). Non-irradiated cells in the same sample were able to germinate as normal, suggesting damage to cells does occur with long term exposure 
to the trapping laser. Similar inhibition of spore germination was observed in 20 conidia trapped for $10-12 \mathrm{~h}$.

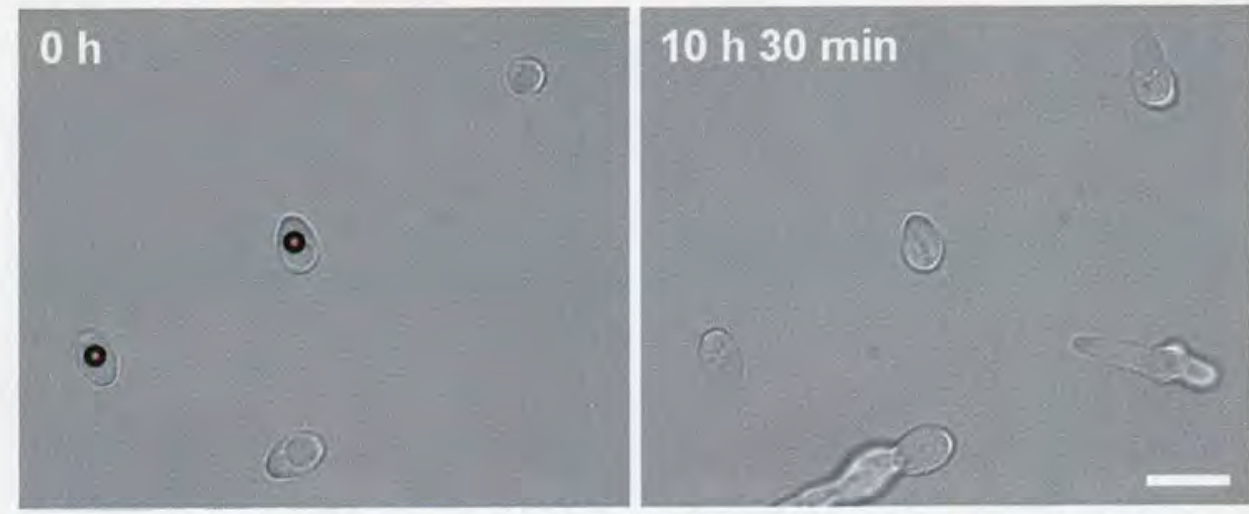

Figure 3.13 Once inoculated two conidia were optically trapped (indicated by the black circles; $0 \mathrm{~h}$ ) and held in place until just prior to the $10 \mathrm{~h} 30 \mathrm{~min}$ time point. Other cells in the same sample germinated as normal. Brightfield imaging, $100 \times$ objective. Bar $=10 \mu \mathrm{m}$.

\subsection{Discussion}

Both ungerminated macroconidia and macroconidial germlings with germ tubes up to $100 \mu \mathrm{m}$ long were optically trapped and moved in a very controlled manner. In each case, a small region of high refractive index within the cell was trapped sufficiently strongly allowing the whole cell to be moved. The addition of a second trapping laser into the system offered more precise control over the orientation of polarised germinated spores and allowed short germlings to be held horizontally in the image plane.

Moving one CAT relative to another using optical tweezers has formed the basis of a novel CAT homing assay developed to investigate cell signalling between CATs (Fleißner et al., 2005; Roca et al., 2005a). In previous studies, conidial germlings were only trapped for up to $1 \mathrm{~min}$ because of concern that longer periods of irradiation might be deleterious to the cells. However, the problem of untrapped CATs tending to drift and rotate, through Brownian motion or convection currents within the sample, changing their relative positions to each other can disrupt an experiment. Here it has been shown that it is possible to continuously trap germinated conidia for periods of at least 25 min without any discernible effects on CAT growth or homing. This long term trapping prevents the problem of conidial germlings drifting relative to each other. Coating coverslips with PVA was found to prevent conidial germlings adhering to the coverslip and this also very much improved the CAT assay. Furthermore, the development of a two-trap optical tweezer system can prevent the drifting of both CATs in a homing assay to achieve greater control over the conidia. 
The development of two different techniques for producing stabilized fixed arrays of cells will be very useful for experimentally analysing cell-cell communication during CAT induction, CAT homing and also the autotropic germ tube avoidance response to other germ tubes and the conidia from which they are derived. None of the extracellular signals involved in these responses have been identified (Read and Roca, 2006; Read, 2007) Arai et al. (2005) also described a similar technique to the Cygel cell immobilisation technique described here. They report developing the use of a thermosensitive gel in combination with a micro-heater to produce a localized gel around single yeast cells trapped near the micro heater.

Multiple-trap optical tweezer systems (see section 1.1.1) also present an exciting and elegant development for the simultaneous trapping of numerous spores in potentially $3 \mathrm{D}$ arrays, but do nothing to reduce the potential for photodamage caused by long term trapping (Rodrigo et al., 2004; Arneborg et al., 2005; Lafong et al., 2006). There have been two published attempts to combine a gelling medium with multiple optical tweezers (Jordan et al., 2005; Akselrod et al., 2006) thereby reducing the irradiation of the cells. Jordan et al. (2005) reported establishing $3 \mathrm{D}$ arrays of $E$. coli cells using holographic optical tweezers. The cells were then permanently fixed in place by setting them in gelatine. Akselrod et al. (2006) were able to trigger the gelation of their medium using a $10 \mathrm{~s}$ burst of UV light to fix 2D and 3D arrays of mammalian and bacterial cells produced with HOTs and time-multiplexed tweezers. Both the gelatine and UV techniques could perhaps be improved by replacing the gelatin with a thermosensitive gel such as the Cygel reported here, or the poly-(Nisopropylacrylamnide) used by Arai et al. (2005), permitting the more selective gelling, and subsequent reversal of the solidified medium back to the liquid state.

The oscillatory recruitment of MAK-2-GFP clearly offers an excellent experimental system for future optical tweezer studies. However, these experiments will require further improvements in the optical tweezer setup. In particular, low dose illumination of the sample during fluorescence imaging will be required to prevent photobleaching of the MAK-2-GFP. Ideally, the optical tweezer manipulation should be done in combination with image deconvolution in order to perform 4D imaging (i.e. $x, y, z$ and time) as shown in my results (Fig. 3.5)

Microfluidic systems (which are increasingly used as part of 'lab-on-a-chip' devices) are an increasingly common technique for use in combination with optical tweezers (Terray et al., 2002; Oakey et al.; 2002; Enger et al., 2004). Laminar flow can allow the analysis of how single cells respond to changes in their external environment. A recent publication 
demonstrated this functionality using the budding yeast Saccharomyces cerevisiae (Eriksson et al., 2007). Cells were optically trapped and carried between environments of different osmolarity and oxidative stress, resulting in changes in cell volume and the nuclear localization of a stress related transcription factor. In the Y-shaped microfluidic cell used here the two channels eventually mixed at the output, but the use of an X-shaped microfluidic cell in the future could permit the separation of the two channels of flow. Using such techniques, stained cells like those in Fig. 3.11 could be easily identified in the collected population of cells.

The $785 \mathrm{~nm}$ laser used in this study did not cause any discernible damage to fungal cells trapped for short to medium periods of time, even when used at its full output power $(70 \mathrm{~mW}$ output power). However extended periods of irradiation was found to permanently prevent germination, although it did not seem to kill the cells. A number of studies have undertaken research to assess the damage caused to cells by optical tweezers (reviewed in section 1.1.1.3). Reducing photodamage to a minimum requires one to judiciously select an optimal combination of laser wavelength, laser power, and limit the duration of exposure to the laser beam, as well as taking into account the type of biological sample being irradiated (Liang et al., 1996). Although cells of filamentous fungi have not been previously used, photodamage studies have been carried out on a wide range of other cell types. A common finding is that $760 \mathrm{~nm}$ light is particularly damaging to cells when compared with $\sim 800 \mathrm{~nm}$ light. The intermediate wavelength of $785 \mathrm{~nm}$, used in the present study, has not been analysed in such detail, but the experiments described here suggest that $N$. crassa cells can tolerate a moderate amount of irradiation, in terms of intensity and duration, at this wavelength. Once germinated, germ tubes and CATs were found to be more tolerant to the laser irradiation. It is plausible that nuclear function is inhibited by long term optical tweezer irradiation, but that this is not sufficient to kill cells. In ungerminated macroconidia all nuclei and cell contents are likely to be exposed to the potentially damaging irradiation, whereas once germinated these organelles may escape direct irradiation due to their distribution within the cells. This would explain why CATs continued to grow and home normally whilst the conidia from which they originated were continuously trapped for $25 \mathrm{~min}$ (Fig. 3.4).

\subsection{Summary}

This chapter demonstrates the following uses for optical tweezers:

- Conidia and conidial germlings can be easily optically trapped, manipulated and moved using relatively low laser powers of a $785 \mathrm{~nm}$ laser 
- A dual beam optical trap allows more precise movement of conidial germlings

- The CAT homing assay was improved by using PVA coated coverglass; conidia with homing CATs can be trapped for 25 min to prevent conidial germlings drifting, without impairing the CAT homing process

- Stable microarrays of spores can be produced either by (1) moving them from a nonsticky PVA coated region of a coverslip to an uncoated region, or (2) fixing them in place using the thermosensitive Cygel gelling agent

- Optically manipulating the positions of adjacent cells relative to each other has immense potential as a very important experimental approach for studying cell-cell signalling in fungi

- Trapping single spores within a population and moving them into different regions of a microfluidic cell allows these individual spores to be selectively stained, treated with chemicals or exposed to a different environmental condition

\subsection{References}

Akselrod, G.M., Timp, W., Mirasaidov, U., Zhao, Q., Li, C., Timp, R., Timp, K., Matsudaria, P., Timp, G., 2006. Laser-guided assembly of heterotypic 3D living cell microarrays. Biophys. J. 91, 3465-3473.

Arai, F., Ng, C., Maruyama, H., Ichikawa, A., El Shimy, H., Fukuda, T., 2005. On chip single-cell separation and immobilization using optical tweezers and thermosensitive hydrogel. Lab on a Chip 5, 1399-1403.

Arneborg, N., Siegumfeldt, H., Andersen, G.H., Nissen, P., Daria, V.R., Rodrigo, P.J., Gluckstad, J., 2005. Interactive optical trapping shows that confinement is a determinant of growth in a mixed yeast culture. FEMS Microbiol. Lett. 245, 155-159.

Berns, M.W., Wright, W.H., Wiegand, S.R., 1991. Laser microbeam as a tool in cell biology. Int. Rev. Cytol. 129, 1-44.

Enger, J., Goksor, M., Ramser, K., Hagberg, P., Hanstorp, D., 2004. Optical tweezers applied to a mirofluidic system. Lab on a Chip 4, 196-200.

Eriksson, E., Enger, J., Nordlander, B., Erjavec, N., Ramser, K., Goksor, M., Hohmann, S., Nystrom, T., Hanstorp, D., 2007. A microfluidic system in combination with optical tweezers for analyzing rapid and reversible cytological alterations in single cells upon environmental changes. Lab on a Chip 7,71-76.

Fleißner, A., Sarkar, S., Jacobson, D.J., Roca, M.G., Read, N.D., Glass, N.L., 2005. The so locus is required for vegetative cell fusion and postfertilization events in Neurospora crassa. Eukaryot. Cell 4, 920-930. 
Jordan, P., Leach, J., Padgett, M., Blackburn, P., Isaacs, N., Goksor, M., Hanstorp, D., Wright, A., Girkin, J., Cooper, J., 2005. Creating permanent 3D arrangements of isolated cells using holographic optical tweezers. Lab on a Chip 5, 1224-1228.

Krylov, S.N., Dovichi, N.J., 2000. Single-cell analysis using capillary electrophoresis: influence of surface support properties on cell injection into the capillary. Electrophoresis 21, 767-773.

Kuo, S.C., Sheetz, M.P., 1992. Optical tweezers in cell biology. Trends Cell Biol. 2, 116-118.

Lafong, A., Hossack, W.J., Arlt, J., Nowakowski, T.J., Read, N.D., 2006. Time-multiplexed LaguerreGaussian holographic optical tweezers for biological applications. Opt. Exp. 14, 3065-3072.

Liang, H., Vu, K.T., Krishnan, P., Trang, T.C., Shin, D., Kimel, S., Berns, M.W., 1996. Wavelength dependence of cell cloning efficiency after optical trapping. Biophys. J. 70, 1529-1533.

Oakey, J., Allely, J., Marr, D.W., 2002. Laminar-flow-based separations at the microscale. Biotechnol. Prog. 18, 1439-1442.

Read, N.D., 2007. Environmental sensing and the filamentous fungal lifestyle. In: Gadd, G.M., Watkinson, S.C., Dyer, P. (Eds.), Fungi in the Environment Cambridge University Press, Cambridge, pp. 38-57.

Read, N.D., Roca, M.G., 2006. Vegetative hyphal fusion in filamentous fungi. In: Baluska, F., Volkmann, D., Barlow, P.W. (Eds.), Cell-Cell Channels Landes Bioscience, Austin, TX.

Roca, M.G., Arlt, J., Jeffree, C.E., Read, N.D., 2005a. Cell biology of conidial anastomosis tubes in Neurospora crassa. Eukaryot. Cell 4, 911-919.

Roca, M.G., Read, N.D., Wheals, A.E., 2005b. Conidial anastomosis tubes in filamentous fungi. FEMS Microbiol. Lett. 249, 191-198.

Rodrigo, P.J., Daria, V.R., Gluckstad, J., 2004. Real-time three-dimensional optical micromanipulation of multiple particles and living cells. Opt. Lett. 29, 2270-2272.

Sheetz, M.P., 1998. Laser Tweezers in Cell Biology. Meth. Cell Biol. 55.

Svoboda, K., Block, S.M., 1994. Biological applications of optical forces. Annu. Rev. Biophys. Biomol. Struct. 23, 247-285.

Terray, A., Oakey, J., Marr, D.W., 2002. Microfluidic control using colloidal devices. Science 296, 1841-1844.

Weber, G., Greulich, K.O., 1992. Manipulation of cells, organelles, and genomes by laser microbeam and optical trap. Int. Rev. Cytol. 133, 1-41.

Wright, G.D., Arlt, J., Poon, W.C., Read, N.D., 2007a. Optical tweezer micromanipulation of filamentous fungi. Fungal. Genet. Biol. 44, 1-13.

Wright, G.D., Arlt, J., Poon, W.C.K., Read, N.D., 2007b. Experimentally manipulating fungi with optical tweezers. Mycoscience 48, 15-19.

Wright, W.H., Sonek, G.J., Tadir, Y., Berns, M.W., 1990. Laser trapping in cell biology. J. Quant. Elec. 26, 2148-2157. 


\section{Chapter 4 - Manipulating organelles within fungal cells with optical tweezers}

\subsection{Introduction}

An unparalleled advantage of optical tweezers is the ability to manipulate organelles within living cells. As has been shown, this can be accomplished without excessive perturbation to cells, if the tweezers are used correctly (sections 1.1.1.3, 3.2.2 and 3.3). For an object to be manipulated it must have a refractive index that is sufficiently different from that of its surroundings. For an object to be trapped by optical tweezers, a necessary condition is that its refractive index (at the wavelength of incident laser light) is greater than that of its surroundings. Conversely, if an object has lower refractive index than its surroundings, it will be repelled by a focussed laser beam. Whilst the former offers much greater control over the positioning of an object within a cell, the latter still allows the user a certain degree of control for moving objects.

The aims of the experimental research described in this chapter were:

- To assess which organelles can be manipulated within cells of $N$. crassa using optical tweezers

- To investigate the tethering of Woronin bodies to septa

- To determine whether ectopically produced intracellular crystals of high refractive index can be used as 'handles' to manipulate intracellular organelles

- To explore how the pattern of hyphal tip growth and branching can be manipulated using either single beam or holographic optical tweezers (HOTs)

Some of the results described in this chapter have been published in Burnham et al. (2007) and Wright et al. (2007a, b). 


\subsection{Results}

\subsubsection{Manipulating organelles of high refractive index}

Organelles of high refractive index (e.g. Woronin bodies) visualized by differential interference contrast (DIC) were readily trapped within living cells (Fig. 4.1; Mov. 4.1). For some organelles it was necessary to hold the organelle in the trap for some time, during which period it seemed that the organelle dissociated from the cytoskeleton, before it became possible to move the organelle around within the cytoplasm. Some Woronin bodies were found to be strongly tethered to the lateral plasma membrane adjacent to septa such that the tweezer trapping forces achievable were insufficient to move them. In order to move organelles within the viscous hyphal cytoplasm it was necessary to move the trap slowly $\left(\sim 1-2 \mu \mathrm{m} \mathrm{s}^{-1}\right)$ so as not to lose the organelle from the trap, a distinct advantage of computer controlled tweezers. Upon the release of an organelle from the trap it was common to observe the organelle 'float' around in the cytoplasm or move with the typical bulk cytoplasmic flow within a hypha (Lew, 2005). Some unidentified, refractile organelles, similar to those described as 'vesicles' by Riquelme et al. (2002), were observed moving tens of micrometers in straight lines within hyphae. These organelles were probably tethered by motor proteins to microtubules. It was often possible to optically trap these organelles but it was apparent that their movement was force-driven. As a result, they were sometimes lost from the trap, particularly when using the trapping laser with low power (data not shown). Moving trapped organelles in a direction that was more-or-less at right angles to the direction in which they were moving along putative microtubules/microfilaments commonly resulted in the organelles being lost from the trap and 'springing back' to the putative cytoskeletal element.
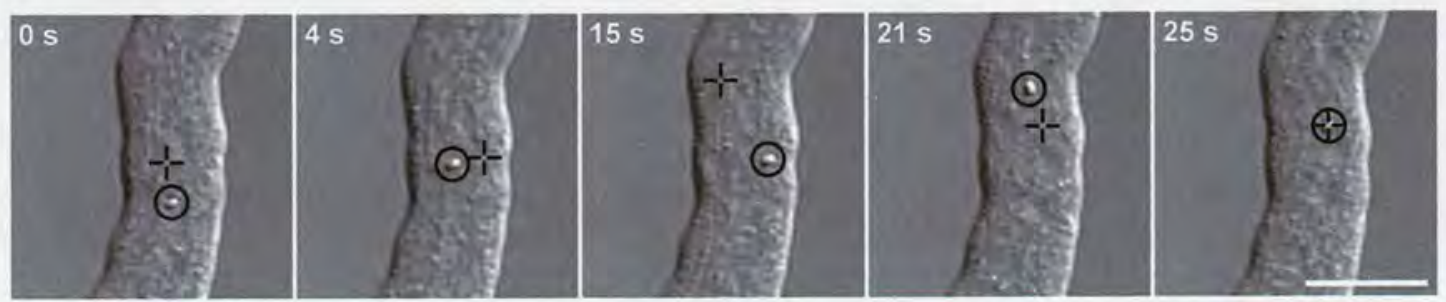

Figure 4.1 Trapping and moving a Woronin body within a hypha of Neurospora crassa. The hyphal region shown was just behind the hyphal tip, and is where Woronin bodies can be found free in the cytoplasm and not, as is more usual, associated with the hyphal cell cortex or septum (Markham and Collinge, 1987; Jedd and Chua, 2000; Tey et al., 2005). The Woronin body could be moved in the direction of the prevailing bulk flow (towards the hyphal tip), laterally across the hypha and against the flow. The circle represents the current position of the laser whilst the cross-hair shows the position which the trap is being moved to. DIC microscopy, $100 \times$ objective. Bar $=10 \mu \mathrm{m}$. (see Mov. 4.1) (from Wright et al., 2007a) 


\subsubsection{Woronin body tethering to septa}

Using a strain of Aspergillus nidulans expressing GFP that labelled Woronin bodies, experiments were performed, in collaboration with Daniel Veith and Reinhard Fischer (University of Karlsruhe, Germany), in an attempt to elucidate the molecules tethering the Woronin bodies in place at the septa. Woronin bodies of $A$. nidulans, although smaller than those of $N$. crassa, could be trapped and repositioned in the hyphal compartments near hyphal tips. However, further back from the hyphal tips, Woronin bodies in A. nidulans were found to be tethered to either side of the septa (Fig. 4.2) because the maximum trapping force of the optical tweezers was insufficient to move them (Mov. 4.2). In order to determine whether microtubules or actin microfilaments were involved in this tethering process, hyphae were treated with Nocodazole $\left(50 \mu \mathrm{g} \mathrm{ml}^{-1}\right)$ or Benomyl $\left(5 \mu \mathrm{g} \mathrm{ml}^{-1}\right)$ to disrupt the microtubules or Cytochalasin D $\left(50 \mu \mathrm{g} \mathrm{ml}^{-1}\right)$ to disrupt the actin microfilaments. In all treatments it was still not possible to pull the Woronin bodies away from the septa (data not shown). A combination of both microtubule and actin microfilament disrupting drugs also did not cause the release of the Woronin bodies (data not shown).
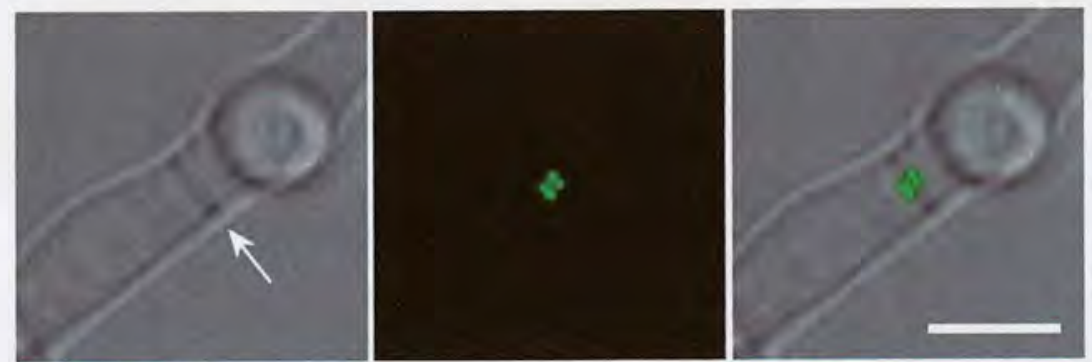

Figure 4.2 Woronin bodies labelled with GFP in germlings of Aspergillus nidulans. A brightfield image of the position of the septa within a germ tube (arrow), a widefield fluorescence image of the GFP labelled Woronin bodies and a merged image of the two are shown. Two Woronin bodies can be seen, one on either side of the septa. 100x objective. Bar $=5 \mu \mathrm{m}$.

\subsubsection{Manipulating ectopically produced crystals within cells}

Using a strain of Schizosaccharomyces pombe, that produced large crystals of polyhedrin (that normally occludes insect virions) within the nucleus, and expressed GFP that labelled the nuclear envelope (Uch2-GFP), experiments to investigate whether these crystals could be used as intracellular 'handles' to manipulate the position of nuclei were performed. This work was done in collaboration with Greg Jedd and Snezhana Oliferenko (Temasek Life Sciences Laboratory, Singapore). The crystals, which resemble Woronin bodies in their appearance, could be trapped and moved within nuclei with the optical tweezers (Fig. 4.3; 
Mov. 4.3). However, it was not possible to use these trapped crystals to reposition the nuclei, suggesting that the nuclei are held in place by the cytoskeleton.

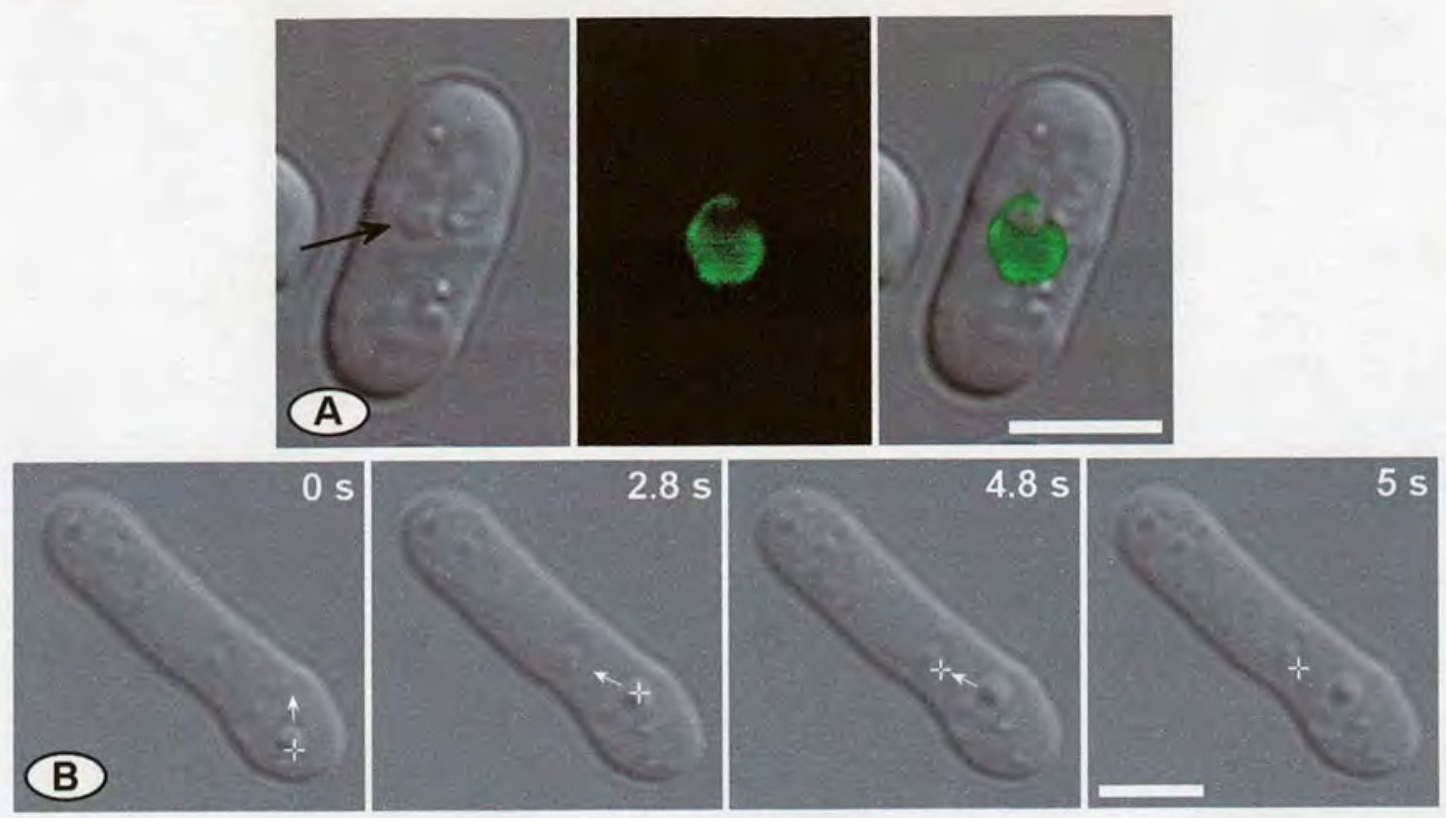

Figure 4.3 Manipulating ectopic crystals of polyhedrin inside the nucleus of Schizosacc. pombe in which the nuclear envelope is labelled with GFP. (A) DIC image acquired on the confocal microscope and merged with a projected $3 D$ confocal $z$-series showing the polyhedrin crystal (black arrow) within the nucleus (green). (B) A failed attempt to manipulate the position of the nucleus using the polyhedrin crystal as a handle. Whilst the crystal's position could be moved within the nucleus (c.f. 0 and $2.8 \mathrm{~s}$ ) when attempts were made to move the crystal further afield it was lost from the trap (c.f. 4.8 and $5 \mathrm{~s}$ ) and the nucleus did not move (data not shown). The white cross-hairs and arrows indicate the position and direction of movement of the trap, respectively. $100 \times$ objective. Bars $=5 \mu \mathrm{m}$. (see Mov. 4.3)

\subsubsection{Manipulating organelles of low refractive index}

Organelles of lower refractive index than their surroundings were repelled by an optical trap. Figures 4.4A-C show vacuoles, which are of lower refractive index than the cytoplasm, being moved around within hyphae whilst being imaged using phase contrast, DIC or fluorescence microscopy. To move the vacuoles, a technique of 'chasing' was employed, much akin to repelling magnets, in which the vacuoles were pushed away from the trap when it got close to them (Fig. 4.4A; Mov. 4.4). Applying the trap directly to a vacuole resulted in it 'popping' out of the trap (Fig. 4.4B; Mov. 4.5). If vacuoles were pressed against the plasma membrane, it was possible to split them in two (Fig 4.4C; Mov. 4.6). 

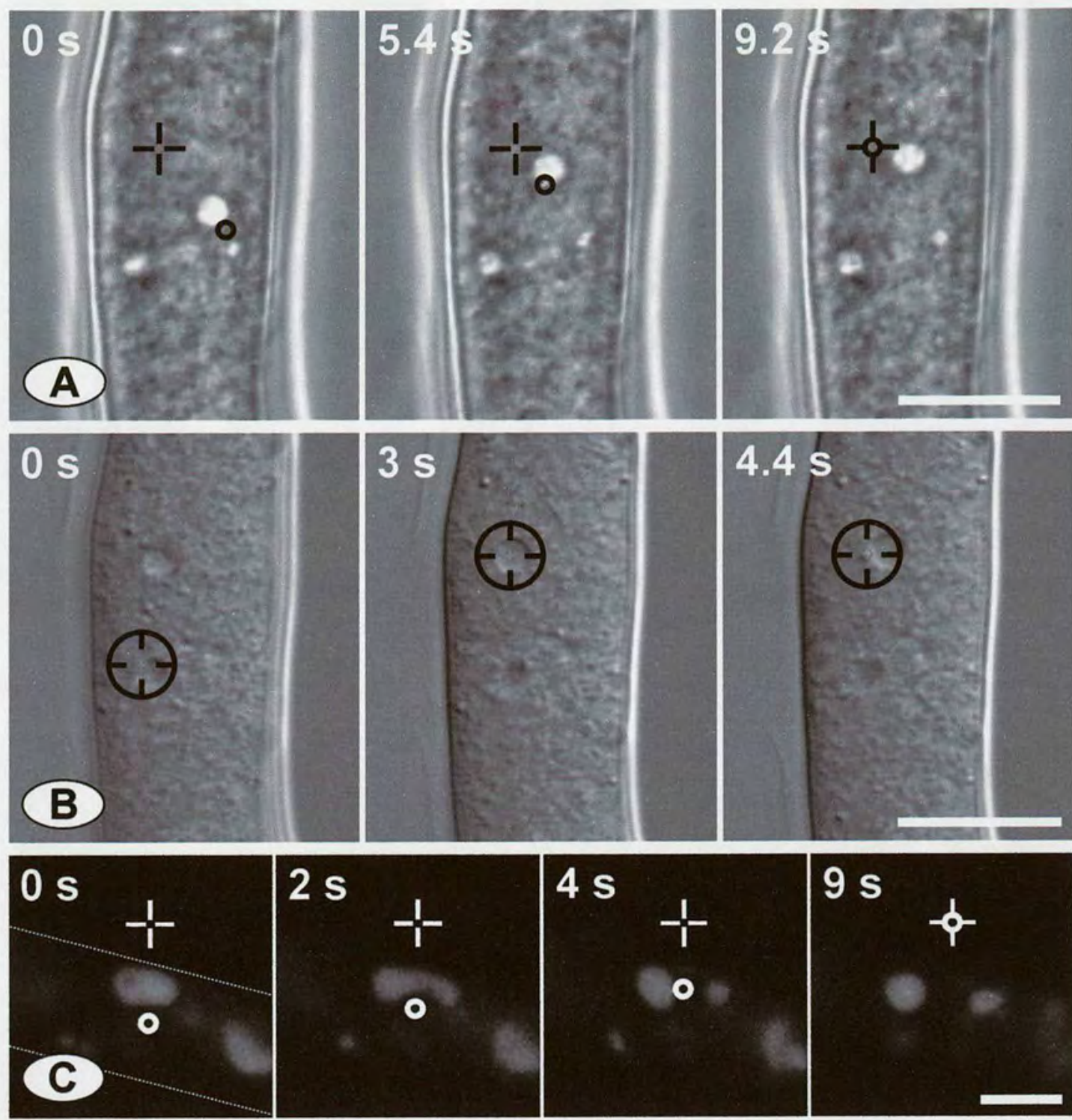

Figure 4.4 Repulsion of vacuoles which have a lower refractive index than their surrounding cytoplasm in Neurospora crassa. (A) Vacuoles are visualised as bright objects (phase light) with phase contrast optics, (B) as depressed 'hollows' with DIC optics, or (C) as brightly fluorescent objects when stained with the dye Oregon Green 488 (DFFDA) and imaged with widefield fluorescence microscopy. If the laser was applied directly to the vacuole they 'popped out' of the trap ( $B$, compare the 3 and $4.4 \mathrm{~s}$ time points). As a result of being repelled, the vacuole could be pushed around the cytoplasm (A). If the vacuoles were pressed against the plasma membrane, it was possible to split them in two (C). The outline of the hypha in (C) is indicated with dotted lines. The circle represents the current position of the laser whilst the cross-hair shows the position to which the trap is being moved to. Bar $=10 \mu \mathrm{m}$ (A and B). Bar $=5 \mu \mathrm{m}(C)$. (see Movs. 4.4 - 4.6) (from Wright et al., 2007a) 


\subsubsection{Manipulating the Spitzenkörper}

\subsubsection{Single Gaussian tweezer experiments}

Using what appeared superficially to be the same 'organelle chasing' technique as demonstrated with vacuoles (section 4.2.3), it was possible to influence the position of the Spitzenkörper within the growing tip using the single $785 \mathrm{~nm}$ optical tweezers (section 2.6.1), as was previously described using $830 \mathrm{~nm}$ light by Bracker et al. (1997). Placing the trap to the side of the Spitzenkörper resulted in the redirection of hyphal tip growth away from the trap (Fig. 4.5; Mov. 4.7). This redirection could be repeatedly used to redirect tip growth over extended periods of time, creating a zigzag pattern of hyphal growth, without perturbing the hyphae (Fig. 4.6; Mov. 4.8).
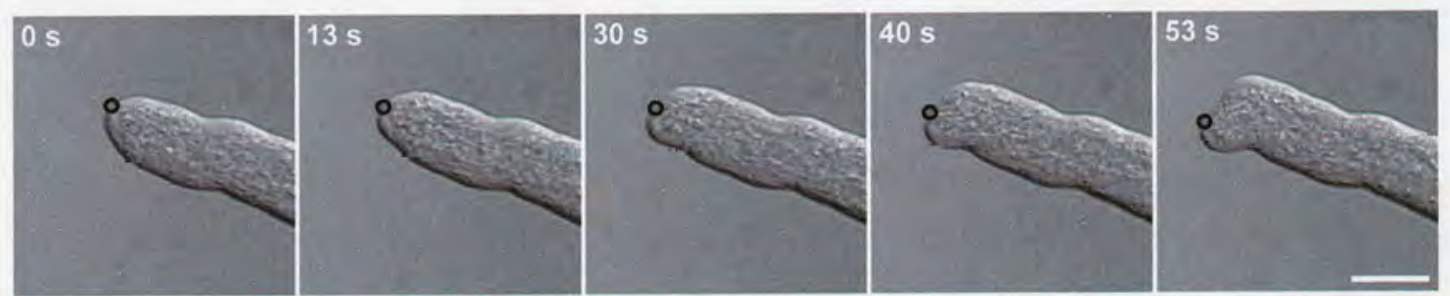

Figure 4.5 Redirection of growth by manipulating the Spitzenkörper. The Spitzenkörper is repelled by the laser. When the laser was located to one side of Spitzenkörper a redirection of the hyphal tip growth away from the trap resulted. In the experiment shown, the trap was repositioned gradually to maintain its position just to the side of the Spitzenkörper as growth progressed. The position of the trap is represented by the circle. DIC microscopy, 100x objective. Bar $=10 \mu \mathrm{m}$. (see Mov. 4.7) (from Wright et al., 2007a)

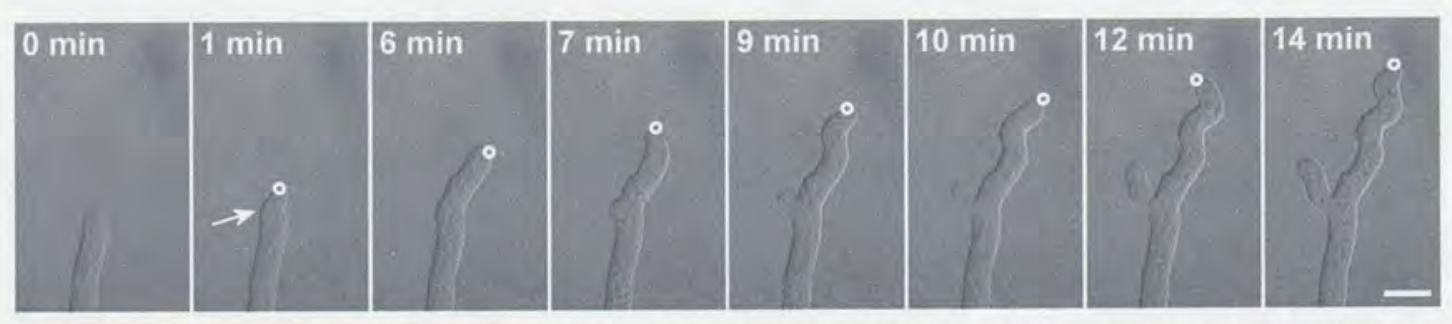

Figure 4.6 Repeated redirection of hyphal tip growth by repulsion of the Spitzenkörper, giving rise to the zigzag growth pattern of the hypha. A branch was formed at the point of initial exposure to the trap (arrow at $1 \mathrm{~min}$ ). The current position of the trap is represented by the circle. DIC microscopy, 100x objective. Bar $=10 \mu \mathrm{m}$. (see Mov 4.8) (from Wright et al., 2007b)

The effects of 532 and $1064 \mathrm{~nm}$ light were also examined. The range of powers needed to cause a change in the pattern of hyphal growth varied largely with wavelength: for $1064 \mathrm{~nm}$ light $150-500 \mathrm{~mW}$, for $785 \mathrm{~nm} 40-70 \mathrm{~mW}$, and for $532 \mathrm{~nm}$ light $1.9-14 \mathrm{~mW}$. For each range stated the minimum power was the lowest to cause an observable effect and the maximum 
was the highest to cause an effect without producing irreparable damage to the hyphae. The laser powers quoted are those that were incident on the back aperture of the microscope objective where the focal spot diameter was approaching the diffraction limit for each wavelength.

Examples of changed growth patterns for each wavelength are shown in Fig. 4.7. The altered growth effect in each case was clearly associated with the proximity of the hyphal tip (and thus Spitzenkörper) to the optical trap. Once the hyphal tips had passed the trap, normal growth resumed back along its original growth direction unless growth was stopped because a hyphal tip was exposed to too high a laser power. In all experiments it was found to be important to judiciously select the minimum laser power necessary to cause a change in the hyphal growth pattern otherwise growth would commonly be stopped.

With all the wavelengths studied, the alteration was short lived, both in time and distance. The usefulness of the techniques described here would be greatly increased if the ability to alter growth could occur passively over relatively extended distances as shown in the proceeding sections.

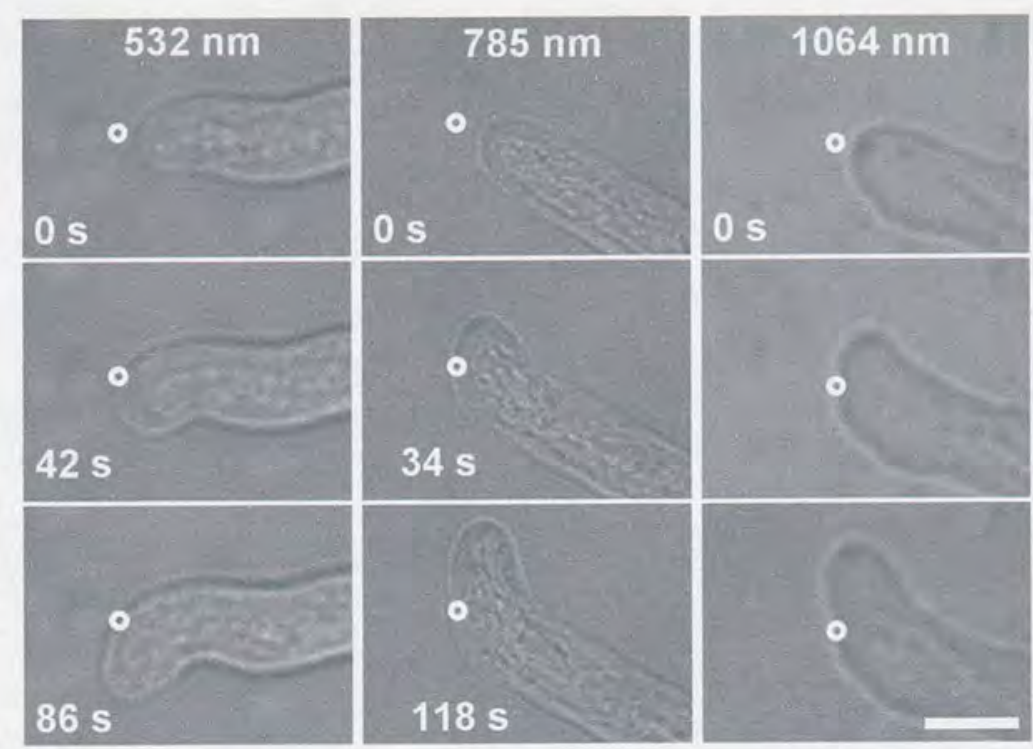

Figure 4.7 Examples of redirecting growth using $532 \mathrm{~nm}, 785 \mathrm{~nm}$ and $1064 \mathrm{~nm}$ single Gaussian beam traps. The power required to cause the effect shown is between 1.9 and 14 $\mathrm{mW}$ at $532 \mathrm{~nm}, 40$ and $70 \mathrm{~mW}$ at $785 \mathrm{~nm}$ and 150 and $500 \mathrm{~mW}$ at $1064 \mathrm{~nm}$. The white circle indicates the positions of the optical traps. Brightfield microscopy, 100x objective. Bar $=10 \mu \mathrm{m}$. (from Burnham et al., 2007) 


\subsubsection{Holographic multiple Gaussian tweezer experiments}

The holographic techniques described (section 2.6.3) allowed arbitrary patterns of multiple tweezers to be created in attempts to alter growth over extended ranges. Figure 4.8 shows the result of allowing a hyphal tip to grow into a curve made from nine individual tweezers.

The results were inconsistent, rarely ending in a tip following the full pattern of multiple optical tweezers. These unsuccessful results generally occurred for one of two reasons. Firstly, the spacing of the tweezers often allowed hyphal tips to grow through the gaps between the tweezers without causing redirection. Secondly, if the hyphae grew through the tweezers their growth could cease due to overexposure to the laser beam.

To minimize these factors the light needed to be evenly distributed along the desired alteration pattern. As discussed, this became possible through the use of holography to create continuous light patterns as shown in the next section.

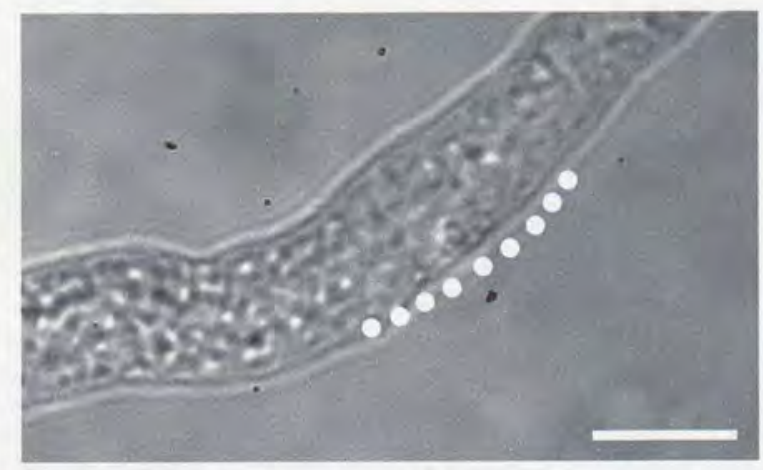

Figure 4.8 Continuous redirection using nine $532 \mathrm{~nm}$ Gaussian traps produced with HOTs. The hyphal tip grew from bottom left to top right. White dots represent the position of each focussed trap. The laser power per tweezer spot was $\sim 2-3 \mathrm{~mW}$. Brightfield microscopy, Plan $100 \times$ objective. Bar $=10 \mu \mathrm{m}$. (from Burnham et al., 2007)

\subsubsection{Holographic continuous light patterns}

Generating single, continuous, $30 \mu \mathrm{m}$ long lines of light at an angle of $\geq 50^{\circ}$ to the direction of growth consistently caused hyphal redirection (Fig 4.9). Inputting parallel laser lines in the software produced a 'channel' of light through which hyphal tips could be guided (Fig. 4.10). In instances where the hypha was wider than the light channel the width of the hypha became narrower as it entered the channel (Fig. 4.11). The first point of interaction between the hyphal tip and the light induced branch formation (Figs. 4.6 and 4.11; and the last images shown in the two series of images in Figs. 4.9 and 4.12). Hyphal branching never 
occurred within a channel bordered by light or from a hypha growing up against a line of light.
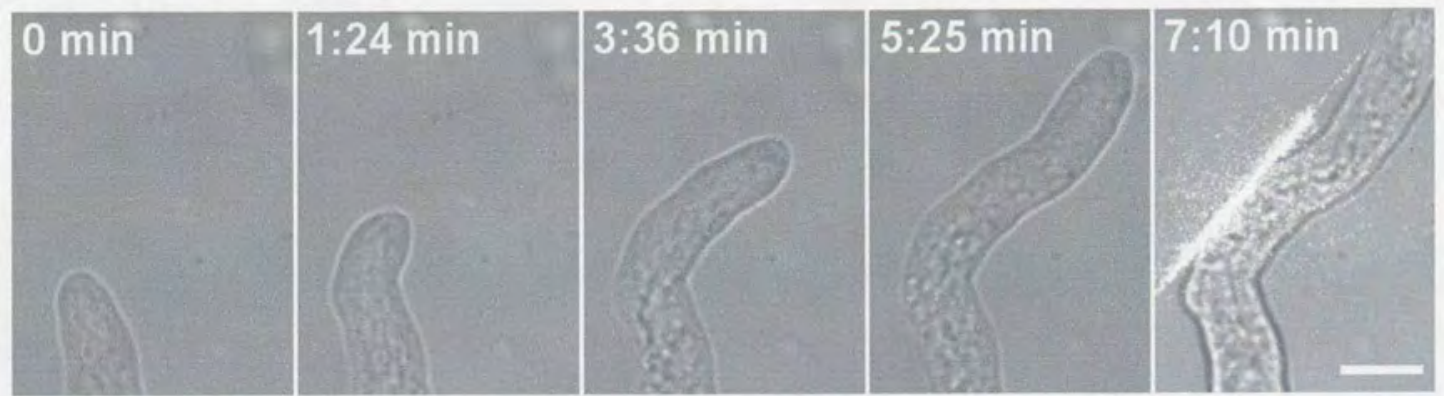

Figure 4.9 Extended hyphal growth redirection using a single continuous line of $532 \mathrm{~nm}$ light introduced at $57^{\circ}$ to the original axis of hyphal growth at time 0 min. The final image in the sequence was captured after the laser filter was removed from its location in front of the camera, allowing visualization of the laser pattern and position. The total laser power spread over the whole pattern was $8.1 \mathrm{~mW}$. Brightfield microscopy, Plan 100x objective. Bar $=10 \mu \mathrm{m}$. (from Burnham et al., 2007)

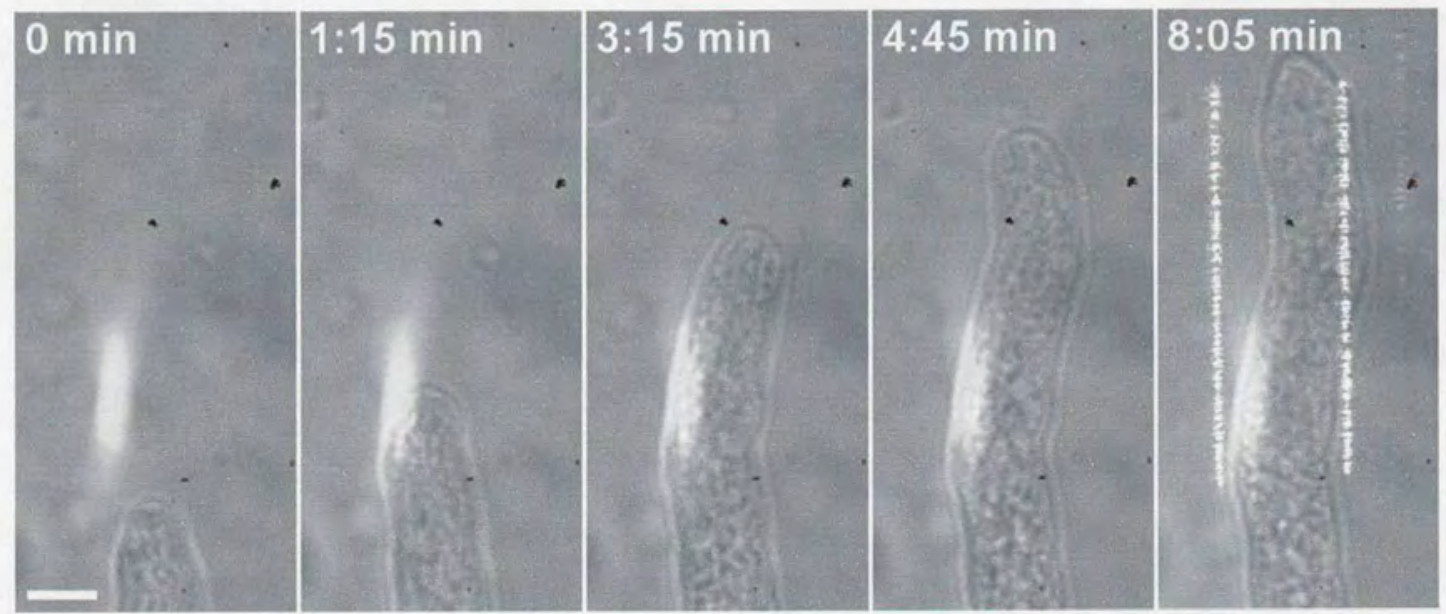

Figure 4.10 Guiding a hyphal tip through a $17 \mu \mathrm{m}$ wide channel bordered by $60 \mu \mathrm{m}$ long lines of light. The final image in the sequence was captured after the laser filter was removed to show the laser pattern and position. The white 'smudge' appearing in each image is an optical artefact. The total laser power spread over the whole pattern was $16.0 \mathrm{~mW}$. Brightfield microscopy, Plan E 100x objective. Bar $=10 \mu \mathrm{m}$. (from Burnham et al., 2007) 


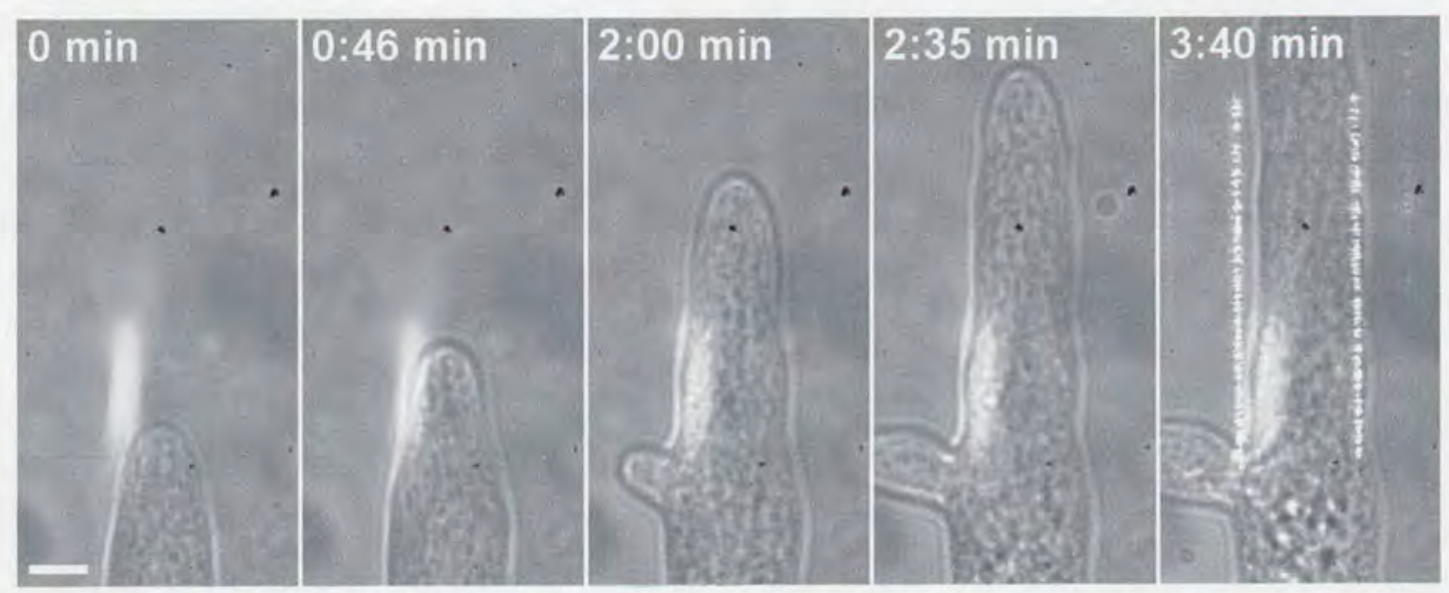

Figure 4.11 Hyphal constriction and branching caused by a relatively thinner channel $(17 \mu \mathrm{m}$ wide) bordered by $60 \mu \mathrm{m}$ long lines of light. The final image in the sequence was captured after the laser filter was removed to show the laser pattern and position. The white 'smudge' appearing in each image is an optical artefact. The total laser power spread over the whole pattern was $9.0 \mathrm{~mW}$. Brightfield microscopy, Plan E 100x objective. Bar $=10 \mu \mathrm{m}$. (from Burnham et al., 2007)

It was simple to rapidly switch between pre-calculated kinoforms and hence optical fields in the focal plane. Figure 4.12 shows two sharp continuous redirections produced using this approach. Having redirected the hyphae at an angle of $45^{\circ}$ by time $4: 44 \mathrm{~min}$, the kinoform was changed to produce a second line of light redirecting the hyphae by another $53^{\circ}$ by time 10:27 min.

It was difficult to calibrate the laser power within the light patterns created. The holograms that produced lines of light parallel to the SLM axes have a higher diffraction efficiency over their diagonal counterparts. Thus, changing between diagonal and parallel lines of the same length also produced a change in intensity. Careful selection of the laser power input into the system was achieved by following a simple procedure. First, an estimate of the power required was made based on the size of the light pattern being produced, and then attempts were made to manipulate hyphal growth. If unsuccessful the power was either increased or decreased depending on whether the hyphae had been unaffected or overly affected, respectively. It was also at times necessary to find a new hyphal tip to experiment on if the power had been overestimated and damaged the hyphal tip. 


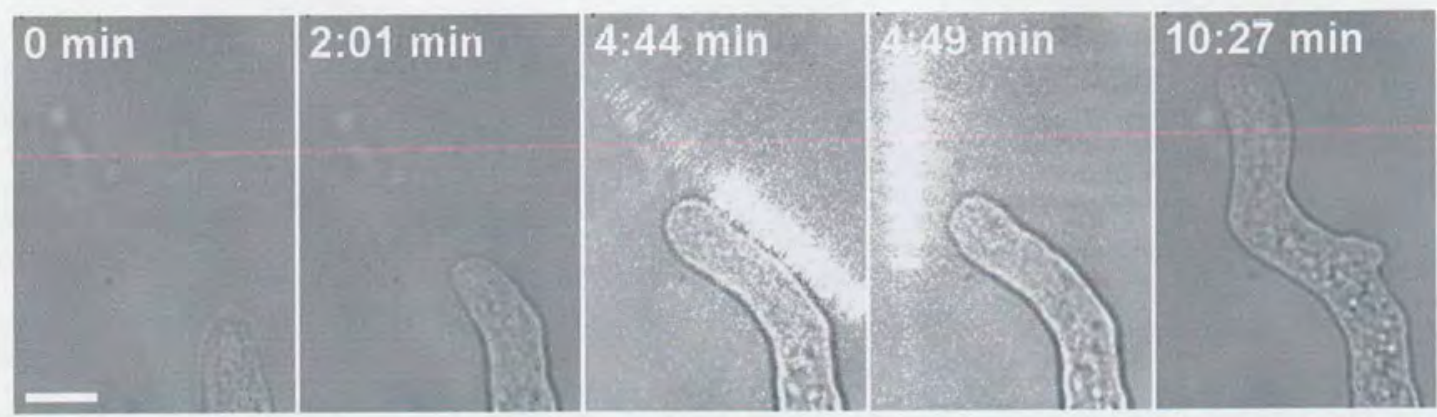

Figure 4.12 Multiple redirections of hyphal growth caused by switching between different kinoforms. The switch in light patterns took place between 4:44 and 4:49 min at which time the laser filter was removed to show laser pattern and position. The total laser power spread over each light pattern was $8.1 \mathrm{~mW}$. Brightfield microscopy, Plan 100x objective. Bar $=10 \mu \mathrm{m}$. (from Burnham et al., 2007)

A pseudowall of light (Fig. 2.8) improved the precision of growth redirection (Fig. 4.13). To produce the pseudowall of light the hologram used for Fig. 4.9 was stacked to produce nine axial planes of light, four each side of the normal focus, each separated by $0.5 \mu \mathrm{m}$. The hyphae consistently followed the edge of the pattern more closely with this optical setup than with those used in the previous experiments described above.

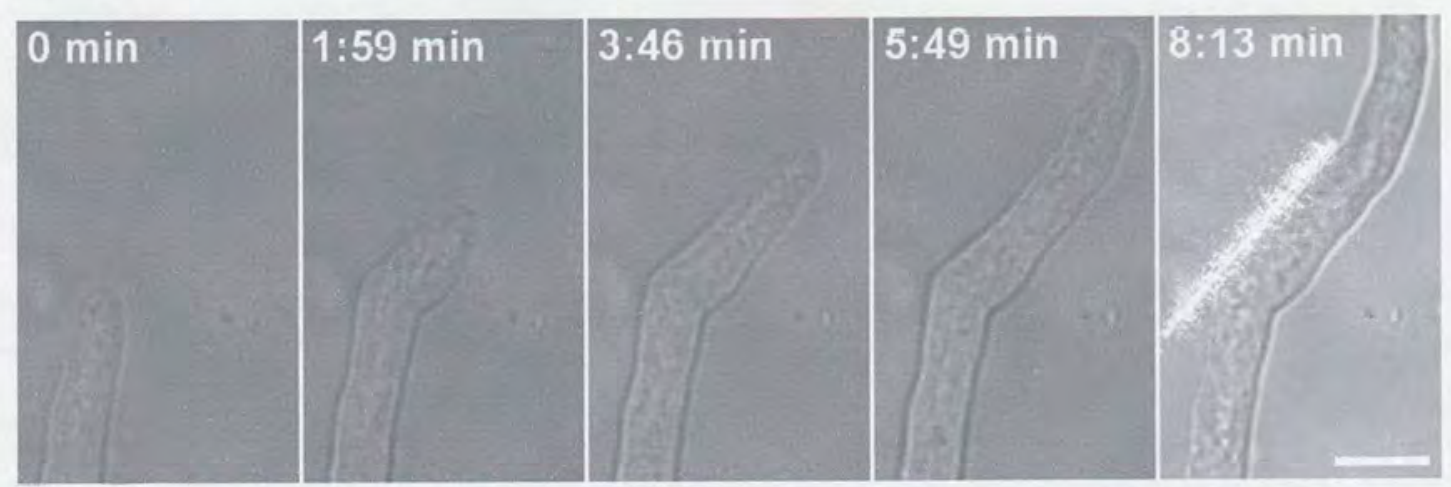

Figure 4.13 Extended hyphal growth redirection using a 3D pseudowall of $532 \mathrm{~nm}$ light. The final image in the sequence was captured after the laser filter was removed to show the laser pattern and position. The total laser power spread over the whole pattern was $8.1 \mathrm{~mW}$. Brightfield microscopy, Plan 100x objective. Bar $=10 \mu \mathrm{m}$. (from Burnham et al., 2007)

\subsection{Discussion}

Fungal organelles of higher refractive index than their surrounding cytoplasm (e.g. Woronin bodies) are usually trapped whilst organelles of lower refractive index (e.g. vacuoles) are repelled by the trapping laser. Essentially organelles that are visible when viewed with brightfield microscopy, which results from their refractive index being different from that of the surrounding cytoplasm, can be manipulated by optical tweezers unless they are tethered 
to other cell components. Conversely organelles that are not visible with microscopy cannot be trapped; this includes the nuclei in $N$. crassa (data not shown). Trapped organelles with high refractive indices can be moved through the naturally viscous cytoplasm at slow speeds. Organelles with lower refractive indices are repelled by the trap and can be pushed or chased around in the cytoplasm. Organelles or particles that are below the resolution of what can be discerned with a light microscope can also be optically trapped if they can exert a force on the photons of the laser beam by absorption, reflection or refraction. In this respect, Bracker et al. (1997) used optical tweezers to generate local high concentrations of secretory vesicles, which individually were not resolvable with the light microscope, to new locations within hyphal tips and, as a result, induced the formation of new branches at those sites. Use of the repulsion method to move organelles of low refractive index is more difficult and less precise but can still be a useful technique for organelle manipulation. It can be helped immensely by the use of computer controlled trap positioning, or if available, the use of multiple traps. This repulsion method also enabled larger spherical vacuoles to be split in two when they were pushed against the plasma membrane.

It was found that it was not always possible to move trapped organelles within hyphae with optical tweezers. Some organelles were clearly tethered to other cell components (e.g. cytoskeletal elements) or the plasma membrane. Indeed, cellular organelles are commonly transported along microtubules and actin microfilaments by means of motor proteins (Steinberg, 2000; Lee and Plamann, 2001; Xiang and Plamann, 2003). The tethering of Woronin bodies was demonstrated in Nectria haematococca by Berns et al. (1992). They described the trapping and moving of Woronin bodies up to a distance of $2 \mu \mathrm{m}$ from a septum before the Woronin bodies were lost from the trap and sprung back to the septum. They inferred that Woronin bodies were tethered close to septa by an unidentified elastic filament which was not visible in the light microscope. Drug treatments, to test whether this tethering was due to actin microfilaments or microtubules, proved that it was neither solely one or other, nor a combination of the two. The Woronin body manipulated in Fig, 4.1 was located close to the hyphal tip in the apical hyphal compartment and was not associated with septa or cell cortex. This Woronin body was untethered and could be readily moved within the hyphal cytoplasm.

Molecular genetic techniques facilitate the production of a crystalline body selectively within an organelle of a living cell. These crystals then have the potential to be subsequently used as an intracellular handle in optical tweezer experiments. The organelle targeting sequences for different organelles proteins can be exploited to direct the accumulation of crystals to an 
organelle of choice. Here, whilst a crystal could be trapped within the nuclei of Schizosacc. pombe it could not then be used to drag the nucleus around within the cell. This suggests that the nucleus is held in place by a force exceeding that generated by the optical tweezers, and this probably involves tethering to the cytoskeleton.

One apparent exception to the theory that organelles of high refractive index can be trapped was that of the Spitzenkörper. As previously reported, the Spitzenkörper in many fungal species is predominantly of high refractive index and is visually phase dark (Girbardt, 1957; Lopez-Franco and Bracker, 1996; Harris et al., 2005). However, it has been previously reported that the Spitzenkörper is repelled by the trapping laser (Bracker et al., 1997; Wright et al., 2005). Closer analysis of the Spitzenkörper of $N$. crassa reveals it to possess a more complex composite structure containing regions of both high and low refractive index (Lopez-Franco et al., 1996; Harris et al., 2005). This may go someway to explaining why the Spitzenkörper is repelled. However, at this stage, it is not possible to say whether the effects of light at the green, near-infrared and infrared wavelengths used are a physical phenomenon (e.g. optical repulsion of the Spitzenkörper, or aversion to localized heating), local intracellular generation of reactive oxygen species (Neuman et al., 1999), or a photoreceptormediated negative phototropism.

The work presented here provides further evidence to support the proposal put forward by Bracker et al. (1997) that the Spitzenkörper is repelled by optical tweezers. It is only when the optical tweezer or light pattern was positioned at the hyphal apex that redirection of tip growth took place. Repeatedly switching the irradiation of the growing hyphal apex from one side to the other resulted in a repeated redirection of the growth axis giving rise to a zigzag pattern of filamentous growth (Bartnicki-Garcia, 2002; Wright et al., 2007b). Figure 4.8 shows that even though the gaps between multiple optical traps were much smaller than the width of the hyphal tips, they were not smaller than Spitzenkörper (Fig. 1.6), which could therefore fit through the gaps without being influenced by the laser. Lines (Fig. 4.9) and 'pseudowalls' (Fig. 4.13) of light were shown to be much more effective at redirecting hyphal grown and when sufficient power was used the hyphal tips were unable to grow across the light barriers.

The reason why hyphal branches were induced when hyphae became constricted by growing into narrow channels bordered by light is unclear. It is possible that the initial perturbation of hyphae by laser irradiation resulted in branch forming. Hyphal branch induction was often observed at the point of initial exposure to a laser trap and branching has been initiated by 
using tweezers to apparently concentrate secretory vesicles within hyphae (Bracker et al., 1997).

It was also shown here that hyphal tip growth can be redirected using optical tweezers with two further wavelengths of light (532 and $1064 \mathrm{~nm}$ ), and the lower the wavelength used, the lower the laser power required to effect these changes. As the reader may have noted ranges of laser powers are quoted at which hyphal growth alteration occurs for each wavelength. This is because the values are largely dependent on the size of the hyphal tips being studied and the patterns being produced. For a given run of experiments an effective power can quickly be found using trial and error with the aim of minimizing the level of irradiation whilst still eliciting a response in the pattern of hyphal growth.

When the range of laser intensities required at the focal plane of the tweezers was calculated, assuming a diffraction limited focus, it was found that lower wavelengths required lower intensities to effect a growth response, indicating a wavelength dependence. The cause of this is unknown.

Using an SLM designed for 400-700 nm operation, digital holography was used to produce fixed patterns of light that can redirect or constrict hyphal growth over extended distances or initiate hyphal branching.

With all the optical methods employed to alter growth, it was possible to use either too little or too much power. If too little power was used then hyphal tip growth was unaffected and the hyphae grew through the light. If too high a laser power was used then hyphal growth ceased, a response common in filamentous fungi exposed to stress (e.g. mechanical or osmotic). In the particular case of single optical tweezers, too high a power could cause hyphae to burst resulting in the cytoplasm leaking from the hyphal tip.

The axial extent of the two-dimensional continuous light patterns produced was very small compared to the actual vertical extent of the hyphal tips $(<18 \mu \mathrm{m})$. The alignment of the system and point of focus are thus highly critical. In order to increase the alignment tolerance, 'pseudowalls' of light were investigated (Fig. 4.13) and found to produce very consistent results in terms of redirecting hyphal growth. Also, the hyphae seemed less perturbed by the walls and followed the barriers much more closely than the single line of light (Fig. 4.9). Two possible reasons for the improvement in results obtained with the 3D pseudowalls of light are as follows. Firstly the same total power was used for both the pseudowalls and lines of light. As the pseudowall was spread between nine individual planes then at any one point the light was less intense, and thus less damaging to the cells. Secondly 
the fact the pseudowalls were extended axially means that given a misalignment in the system there was a higher chance of the hyphal tip and Spitzenkörper being coplanar with the light field. One of the main advantages of holographic beam shaping is its ability to produce light patterns with axial extent, unlike, for example, acousto-optical deflectors (AODs).

\subsection{Summary}

- Woronin bodies, and other organelles of high refractive index relative to the cytosol, can be trapped and their position manipulated, unless they are tethered, within cells

- Vacuoles, and other organelles of low refractive index relative to the cytosol, are repelled by the laser and their position within the cell can be manipulated by a process akin to repelling magnets

- Ectopically produced crystals can be manipulated within fungal cells and have potential for moving non-tethered organelles in the future

- Single traps, multiple traps and continuous holographic light patterns can be used to control the direction of hyphal growth by manipulating the position of the Spitzenkörper within hyphal tips. The holographic light patterns can achieve continuous redirection over extended ranges

- Pseudowalls of light, created using HOTs, were shown to increase the efficiency of hyphal growth redirection

\subsection{References}

Bartnicki-Garcia, S., 2002. Hyphal tip growth: outstanding questions. In: Osiewacz, H.D. (Ed.), Molecular Biology of Fungal Development Marcel Dekker, New York, pp. 29-58.

Berns, M.W., Aist, J.R., Wright, W.H., Liang, H., 1992. Optical trapping in animal and fungal cells using a tunable, near-infrared titanium-sapphire laser. Exp. Cell Res. 198, 375-378.

Bracker, C.E., Murphy, D.J., Lopez-Franco, R., 1997. Laser microbeam manipulation of cell morphogenesis in growing fungal hyphae. Proc. SPIE 2983, 67-80.

Burnham, D.R., Wright, G.D., Read, N.D., McGloin, D., 2007. Holographic and single beam optical manipulation of hyphal tip growth in filamentous fungi. J. Opt. A: Pure Appl. Opt. 9, S172-S179.

Girbardt, M., 1957. Der Spitzenkörper Von Polystictus versicolor (L). Planta 50, 47-59.

Harris, S.D., Read, N.D., Roberson, R.W., Shaw, B., Seiler, S., Plamann, M., Momany, C., 2005. Polarisome meets Spitzenkörper: microscopy, genetics, and genomics converge. Eukaryot. Cell 4, 225-229. 
Jedd, G., Chua, N.H., 2000. A new self-assembled peroxisomal vesicle required for efficient resealing of the plasma membrane. Nat. Cell Biol. 2, 226-231.

Lee, I.H., Plamann, M., 2001. Microtubules and molecular motors. In: Howard, R.J., Gow, N.A.R. (Eds.), The Mycota: Biology of the Fungal Cell, vol. VIII Springer-Verlag, Berlin, pp. 225-241.

Lew, R.R., 2005. Mass flow and pressure-driven hyphal extension in Neurospora crassa. Microbiology 151, 2685-2692.

Lopez-Franco, R., Bracker, C.E., 1996. Diversity and dynamics of the Spitzenkörper in growing hyphal tips of higher fungi. Protoplasma 195, 90-111.

Markham, P., Collinge, A.J., 1987. Woronin bodies of filamentous fungi. FEMS Microbiol. Rev. 46, $1-11$.

Neuman, K.C., Chadd, E.H., Liou, G.F., Bergman, K., Block, S.M., 1999. Characterization of photodamage to Escherichia coli in optical traps. Biophys. J. 77, 2856-2863.

Riquelme, M., Roberson, R.W., McDaniel, D.P., Bartnicki-Garcia, S., 2002. The effects of ropy-1 mutation on cytoplasmic organization and intracellular motility in mature hyphae of Neurospora crassa. Fungal. Genet. Biol. 37, 171-179.

Steinberg, G., 2000. The cellular roles of molecular motors in fungi. Trends Microbiol. 8, 162-168.

Tey, W.K., North, A.J., Reyes, J.L., Lu, Y.F., Jedd, G., 2005. Polarized gene expression determines Woronin body formation at the leading edge of the fungal colony. Mol. Biol. Cell 16, 2651-2659.

Wright, G.D., Arlt, J., Poon, W.C., Read, N.D., 2007a. Optical tweezer micromanipulation of filamentous fungi. Fungal. Genet. Biol. 44, 1-13.

Wright, G.D., Arlt, J., Poon, W.C.K., Read, N.D., 2005. Measuring fungal forces with optical tweezers. Proc. SPIE 5930, F1-F7.

Wright, G.D., Arlt, J., Poon, W.C.K., Read, N.D., 2007b. Experimentally manipulating fungi with optical tweezers. Mycoscience 48, 15-19.

Xiang, X., Plamann, M., 2003. Cytolskeleton and motor proteins in filamentous fungi. Curr. Opin. Microbiol. 6, 628-633. 


\section{Chapter 5 - Manipulating microspheres with optical tweezers as experimental tools to study fungal cells}

\subsection{Introduction}

Polystyrene beads and other types of transparent microspheres can usually be efficiently trapped, and can provide powerful experimental tools in live-cell studies. They have been commonly used in optical tweezer studies for the demonstration of tweezer functionality (Ashkin et al., 1986), when precise measurements are required (Kuo, 2001) and as handles for biological molecules (Cluzel et al., 1996; Baumann et al., 2000; Greulich, 2005). Microspheres of various sizes, composition and with a plethora of surface and physical properties are commercially available (e.g. from Bangs Laboratories Inc., www.bangslabs.com), so the potential for novel experimentation is vast.

The aims of the experimental research described in this chapter were:

- To calibrate the trapping forces produced by the single trap optical tweezer system

- To determine whether optically trapped beads can be used to measure hyphal growth forces

- To determine whether hyphae can be mechanostimulated with trapped beads

- To develop a technique to deliver chemicals to localized regions of hyphae

Some of the results described in this chapter have been published in Wright et al. (2005; 2007) 


\subsection{Results}

\subsubsection{Measuring fungal forces}

\subsubsection{Trapping force calibration}

A bead situated at the centre of a laser trap experiences no net force. As it is displaced from the trap centre, a restoring force develops that is initially proportional to the displacement, i.e. the trap acts as an ideal (Hooke's law ${ }^{1}$ ) spring. Eventually, however, the trap 'softens' and the force decreases to zero: the bead escapes the trap beyond a maximum displacement (see section 5.2.1.3, Fig. 5.6). Precise measurement of the position of the trapped bead (e.g. using a quadrant photon detector [QPD]) allows the determination of the force exerted on the bead (Sheetz, 1998; Gittes and Schmidt, 1998; Wilson et al., 2005). On the other hand, a good estimate of the forces involved can conveniently be obtained by measuring the maximum trapping force, which increases linearly with the incident laser power. This was measured for the $785 \mathrm{~nm}$ single optical tweezer system (section 2.6.1) by trapping a bead at a given laser power and then dragging it through a liquid of known viscosity at increasing velocity, using a motorized stage, until it escaped from the trap (Mov. 5.1). Applying Stoke's law (Eqn. 5.1) to calculate the drag at the 'escape speed', allowed the calculation of the maximum trapping force at that laser power. Tweezer calibration was performed for $1 \mu \mathrm{m}$ and $4 \mu \mathrm{m}$ polystyrene beads (Fig. 5.1).

$$
F=6 \pi \cdot \eta \cdot a \cdot v
$$

Equation 5.1

Where $\eta$ is the viscosity $\left(0.001025 \mathrm{~N} \mathrm{~m}^{-2} \mathrm{~s}\right.$ for water at $\left.20^{\circ} \mathrm{C}\right), a$ is the radius of the bead, and $v$ is the velocity measured.

The laser powers that could be calibrated were limited by the maximum velocity of the motorised stage. The resultant calibration graph shows a good linear relationship between the laser power and the trapping force, thereby allowing extrapolation to higher laser powers (Fig. 5.1). A maximum trapping force of $\sim 11 \mathrm{pN}$ for $1 \mu \mathrm{m}$ beads and $\sim 19 \mathrm{pN}$ for $4 \mu \mathrm{m}$ polystyrene beads was measured.

\footnotetext{
${ }^{1}$ Hooke's law: named after Robert Hooke (1635-1703), an approximation that states that, for an elastic body, the stress (force causing a deformation) is directly proportional to the strain (the amount of deformation).
} 


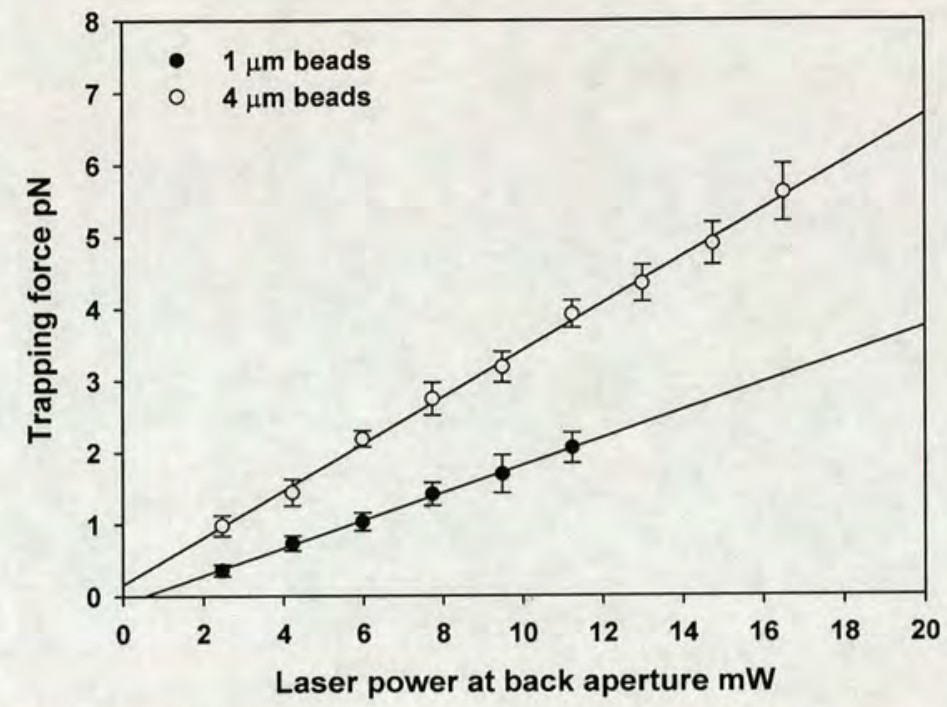

Figure 5.1 Force calibration of the optical tweezer system for 1 and $4 \mu \mathrm{m}$ beads. The scatter plot was subjected to 'least square' regression to produce the best-fit lines, which can be extrapolated to the full range of laser power available (up to $70 \mathrm{~mW}$ ). The best-fit lines should, in theory, pass through the origin. The fact that they do not demonstrates the inherent inaccuracy of using the escape force method for optical tweezer calibration. (from Wright et al., 2005)

\subsubsection{Hyphal growth forces}

Once the trapping force of the tweezers was calibrated for a given laser power and size of polystyrene bead, attempts to measure hyphal growth forces were made. As shown in Fig. 5.2 and Mov. 5.2, the growth force of a leading hypha of $N$. crassa was sufficient to push a $4 \mu \mathrm{m}$ polystyrene bead out of the trap even when using the highest laser power ( $70 \mathrm{~mW}$; a trapping force of $\sim 19 \mathrm{pN}$ ), as was expected based on previous reports which have shown vegetative hyphae to produce forces in the $\mu \mathrm{N}$ range (Money, 2001; Money et al., 2004). Some initially unexpected contradictory results were obtained when using $1 \mu \mathrm{m}$ beads trapped in this way. With the full laser power $(\sim 11 \mathrm{pN})$, leading hyphae were found to be unable to push $1 \mu \mathrm{m}$ beads from the trap (Fig. 5.3; Mov 5.3). Instead their growth slowed and the hyphae continued to grow, but rather than pushing the trapped bead out of the way they grew around it. In contrast, however, $1 \mu \mathrm{m}$ beads trapped with $20 \mathrm{~mW}$ laser power $(\sim 3 \mathrm{pN})$ were pushed out of the trap by the growing hyphae, which continued to grow straight (Fig. 5.4; Mov 5.4). It is significant, and inevitable, that the hyphal tips will grow directly into the laser as it pushes on a $1 \mu \mathrm{m}$ bead. A problem associated with this is that the laser beam can interfere with the Spitzenkörper by repelling it (as shown in section 4.2.4). Therefore, in instances where the tip appears to grow around the trapped bead (Fig. 5.3; Mov 5.3) it seems that it was actually the repositioning of the Spitzenkörper by the laser that 
caused the redirection of hyphal growth. The lower laser power of $20 \mathrm{~mW}$ was insufficient to cause growth redirection (Fig. 5.4; Mov. 5.4). In section 4.2.3.1 it was shown that a laser power $>40 \mathrm{~mW}$ at $785 \mathrm{~nm}$ was required to redirect hyphal growth.
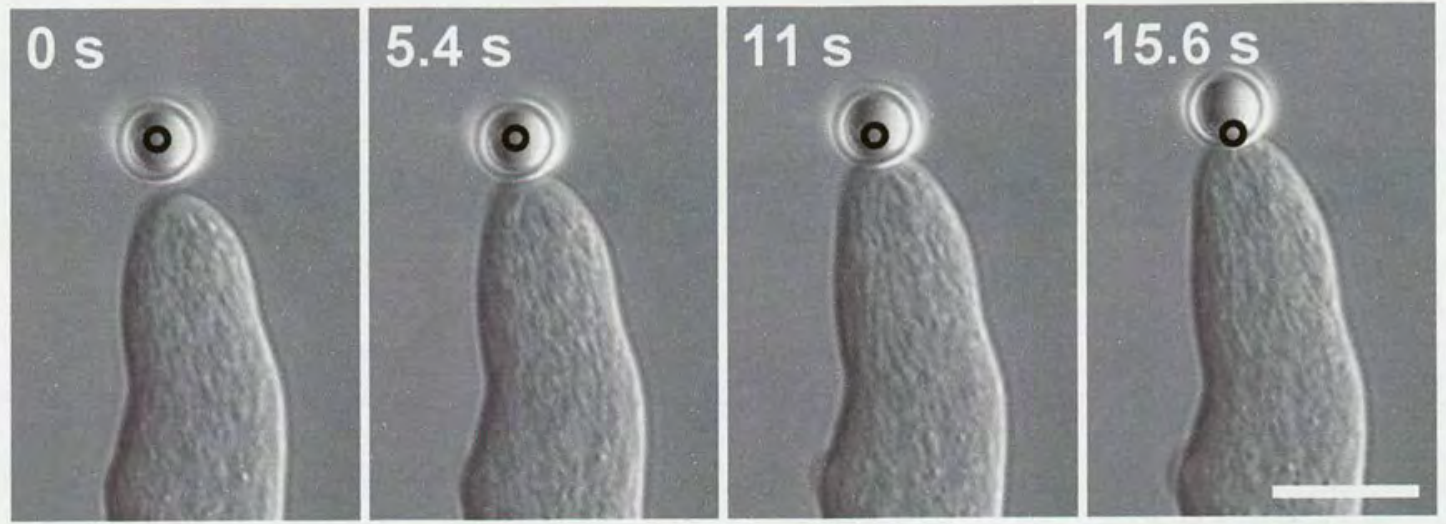

Figure 5.2 Inability to halt the growth of a leading hypha with an optically trapped $4 \mu \mathrm{m}$ polystyrene bead. The tip was able to push the bead out of the trap. The highest output laser power $70 \mathrm{~mW}$ was used in this experiment, which equates to a trapping force of $19 \mathrm{pN}$. The circle represents the position of the laser. DIC microscopy, 100x objective. Bar $=10 \mu \mathrm{m}$. (from Wright et al., 2007) (see Mov. 5.2)
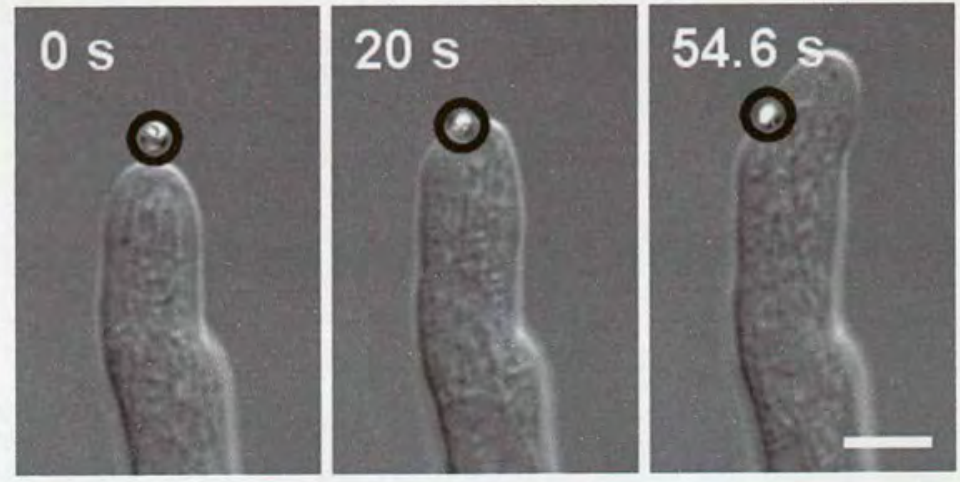

Figure 5.3 Trapping a $1 \mu \mathrm{m}$ bead with $70 \mathrm{~mW}$ laser power $(11 \mathrm{pN})$ caused hyphae to grow around the trapped bead. The redirection, however, was a result of the repositioning of the Spitzenkörper by the laser, as opposed to the hyphal tip being unable to exert sufficient force to push the bead from the trap. The circle represents the position of the laser. DIC microscopy, $100 \times$ objective. Bar $=5 \mu \mathrm{m}$. (see Mov. 5.3) (from Wright et al., 2005) 


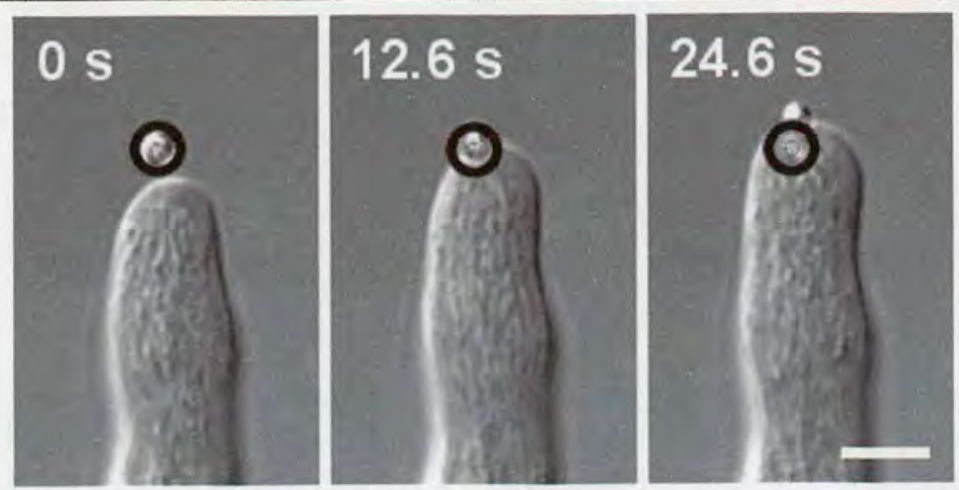

Figure 5.4 A $1 \mu \mathrm{m}$ bead trapped with $20 \mathrm{~mW}$ of laser power $(3 \mathrm{pN})$ was pushed out of the trap by leading hyphae. The circle represents the position of the laser. DIC microscopy, 100x objective. Bar $=5 \mu \mathrm{m}$. (see Mov. 5.4) (from Wright et al., 2005)

\subsubsection{Germ tube growth forces}

Performing the same experiments using germ tubes yielded very different results (Fig. 5.5; Mov. 5.5). The tips of germ tubes, which grow much slower than leading hyphae, became swollen upon contact with $4 \mu \mathrm{m}$ beads trapped at full laser power, and then stopped growing. This suggests that germ tubes produce a smaller growth force than leading hyphae. Upon removal of the obstructing bead, growth resumed at the germ tube tip, leaving a subapical swelling (Fig. 5.5; Mov. 5.5).
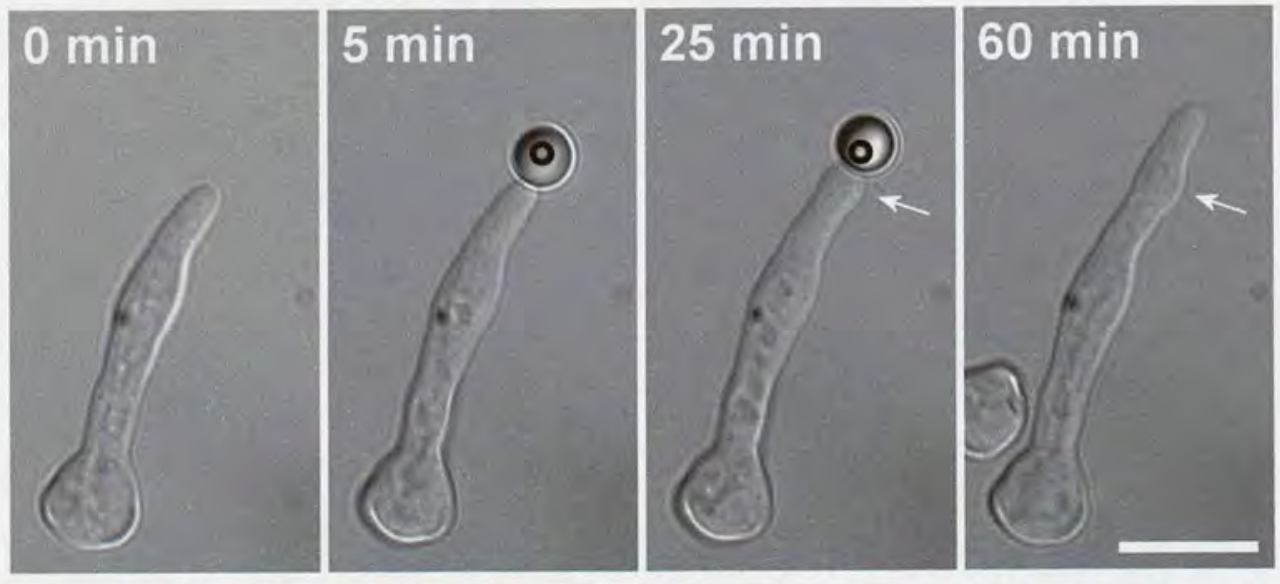

Figure 5.5 Inhibition of germ tube growth with an optically trapped $4 \mu \mathrm{m}$ bead used as an obstacle in front of a germ tube. Note that upon first making contact with the bead the germ tube tip began to displace the bead slightly (at $5 \mathrm{~min}$ ), but was unable to push it out of the trap. The tip then proceeded to swell (arrow at $25 \mathrm{~min}$ ). When the obstacle was removed growth resumed at the germ tube tip, leaving a subapical swelling (arrow at $60 \mathrm{~min}$ ). The highest output laser power $(70 \mathrm{~mW})$ was used in this experiment, which equates to a trapping force of $19 \mathrm{pN}$. The circle represents the position of the laser. DIC microscopy, 100x objective. Bar $=10 \mu \mathrm{m}$. (from Wright et al., 2007) (see Mov. 5.5)

The germ tube tip initially displaced the bead from the trap by a measurable distance $(0.8 \mu \mathrm{m}$; Fig. 5.5, $5 \mathrm{~min})$. Using data, such as that shown in Mov. 5.1, the spring constant for 
a $4 \mu \mathrm{m}$ bead in the trap was estimated (the measured escape force divided by the measured displacement just prior to escape). The displacement achieved by the germ tube tip equates to a force of $11.4 \mathrm{pN}$. However, due to the methods used for calculating the spring constant, this figure will be an underestimate (Fig. 5.6). It suggests that the tip shown could produce a force of between 11.4 and $19 \mathrm{pN}$.

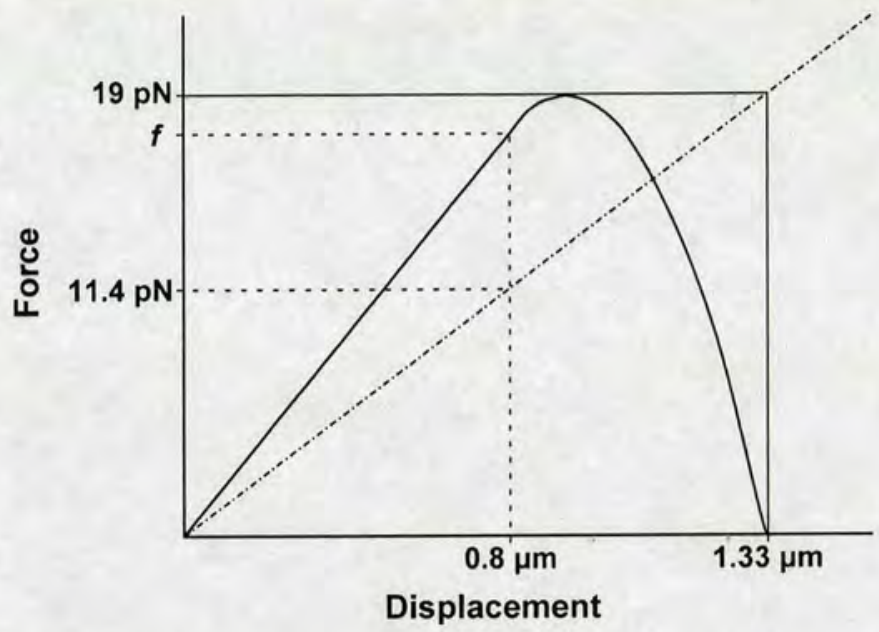

Figure 5.6 A schematic plot (continuous curve) of the force experienced by a bead (vertical axis) held in an optical trap as a function of its displacement from the trap centre (horizontal axis). At the trap centre the force is zero; the force initially increases linearly with displacement (i.e. it acts like a spring obeying Hooke's law). Eventually, at large displacements, the force-displacement relation departs from linearity. The trap softens, until the force reduces to zero. The germ tube in Fig. 5.5 displaces the bead by from the trap centre by $0.8 \mu \mathrm{m}$. To calculate the force it is exerting, which is given by $f$ in this sketch, the 'spring constant', i.e. the slope of the linear portion of the force-displacement curve, is needed. To estimate this quantity, the measured escape force of a $4 \mu \mathrm{m}$ bead (from Stokes law, Eqn. 5.1; cf. Fig. 5.1) was divided by the measured displacement just prior to escape (from Mov. 5.1). This gives the slope of the dotted straight line, which is less than that of the linear portion of the true force-displacement curve, thus providing a lower value for the spring constant. Using this value, an estimate that the germ tube is exerting a force of $11.4 \mathrm{pN}$ can be made. The true value, $f$, will be higher, but below $19 \mathrm{pN}$, the escape force at this laser power.

\subsubsection{Mechanostimulation of fungal cells}

Whilst germ tubes reacted to the mechanostimulus presented by a stationary obstacle, by swelling at their tips (Fig. 5.5) hyphae did not (Figs. 5.2. and 5.4). Another method of applying a mechanostimulus to growing hyphal tips was attempted. This involved using a $10 \mu \mathrm{m}$ bead to repeatedly hit growing tips of leading hyphae by moving the trapped bead at high speeds (up to $40 \mu \mathrm{m} \mathrm{s}^{-1}$ ) back and forth against the hyphal tip. The hyphae treated in this way continued to maintain a uniform linear rate of extension but some slight redirection of growth was sometimes observed following the time point at which the stimulus was applied (Fig. 5.7; Mov. 5.6) 

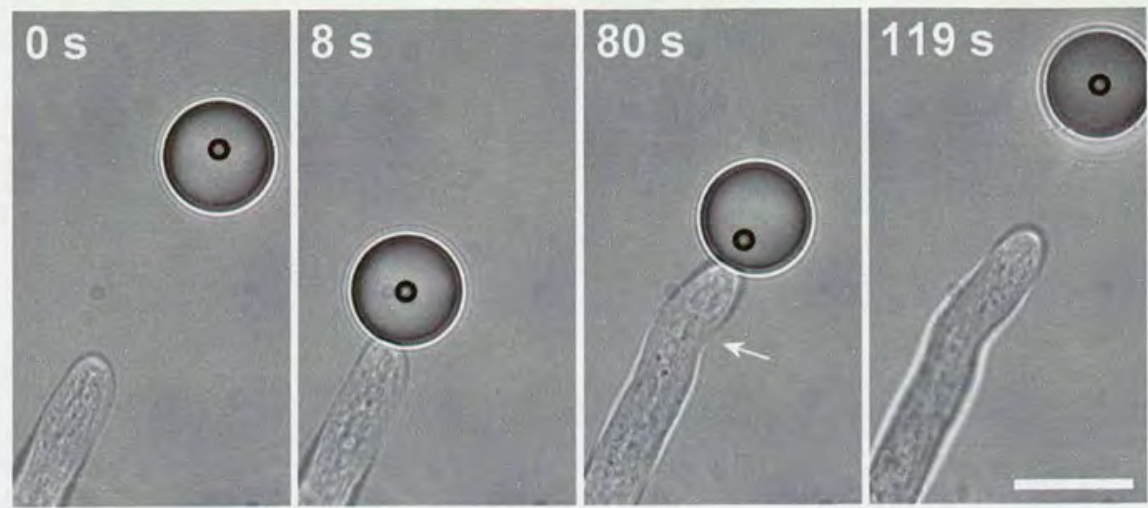

Figure 5.7 Effect of repeatedly hitting a tip of a growing vegetative hypha with a $10 \mu \mathrm{m}$ polystyrene bead. Note that the hypha continued to grow (with an extension rate of $7.7 \mu \mathrm{m}$ $\mathrm{min}^{-1}$ ) throughout the period of mechanostimulation but there was a slight redirection of growth during the time (arrow at $80 \mathrm{~s}$ ) that the hypha was stimulated. The circle represents the position of the laser. Brightfield microscopy, 100x objective. Bar $=10 \mu \mathrm{m}$. (from Wright et al., 2007) (see Mov. 5.6)

\subsubsection{Localised delivery of chemicals to cells}

Optical tweezers were used to deliver chemicals to localized cellular regions. In Fig. 5.8A latrunculin-B, a drug that inhibits actin polymerisation (Spector et al., 1983), was delivered to a hyphal tip selectively using a group of three porous silica beads. The extension rate of this hypha was dramatically reduced and as it continued to grow slowly it underwent significant swelling. The growth of a neighbouring hypha up to $\sim 300 \mu \mathrm{m}$ away continued normally during the $25 \mathrm{~min}$ period of this experiment (Fig. 5.8B). The porous beads, having been soaked in latrunculin-B, were added to the edge of the agar block then trapped and taken to the hyphal tip through the liquid growth medium between the agar block and the coverslip. 

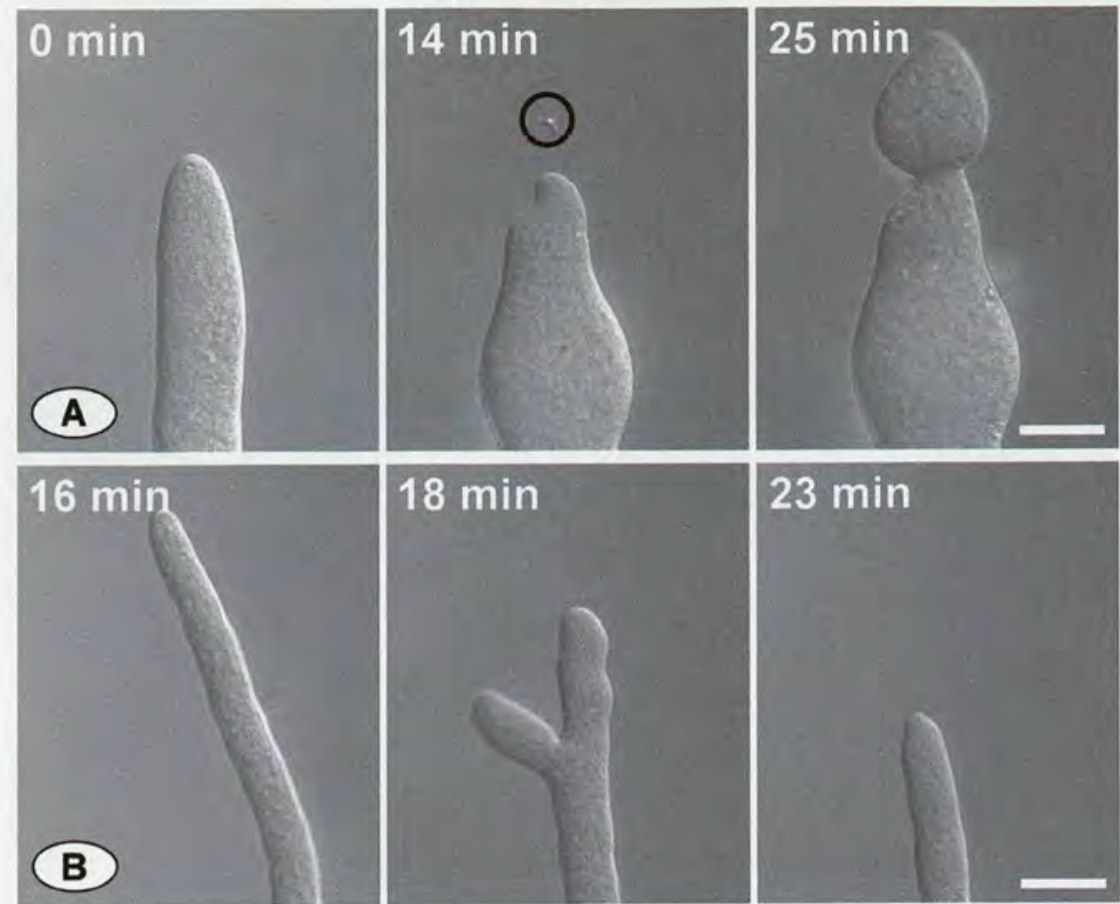

Figure 5.8 Using trapped porous beads to deliver chemicals to a localized region of a cell. (A) Delivery of a localized dose of latrunculin-B to a hyphal tip from a group of 3 porous silica beads. The drug, which disrupts actin polymerisation, has caused the hyphal tip growth to be significantly inhibited and was accompanied by a gross swelling of the hyphal tip region. (B) A neighbouring hyphae, which was $\sim 300 \mu \mathrm{m}$ away from the treated hyphae, was unaffected during the period of this experiment, and is shown branching normally at time $18 \mathrm{~min}$. In both (A) and (B) the microscope stage has been moved in order to keep the growing hyphal tips within the field of view. The circle represents the position of the laser. DIC microscopy, 100x objective. Bar $=10 \mu \mathrm{m}$. (from Wright et al., 2007)

\subsection{Discussion}

It was found that the trapping forces which can be generated by the $785 \mathrm{~nm}$ laser tweezer system with $4 \mu \mathrm{m}$ beads are in the range of $1-19 \mathrm{pN}$. Using trapped beads it was shown that the growth force of leading hyphae of $N$. crassa is sufficient to push a $4 \mu \mathrm{m}$ polystyrene bead out of a trap even when using the highest laser power (equivalent to a trapping force of $19 \mathrm{pN}$ ). This is consistent with results obtained by Money et al. (2004) who estimated, from measurements using a miniaturised strain gauge, that vegetative hyphae generate growth forces several orders of magnitude greater than those that can be measured with optical tweezers. However, the extension of germ tubes was inhibited by a bead of the same size trapped with the same force. This indicates that the growth force generated by a germ tube is significantly less than that of leading hypha. For this application $4 \mu \mathrm{m}$ beads proved better than smaller ones (e.g. $1 \mu \mathrm{m}$ beads) because their use prevented problem of directly irradiating growing hyphal tips and potentially influencing the behaviour of the Spitzenkörper with the laser trap (section 4.2.4; Bracker et al., 1997). By calculating the 
spring constant for the trap, the displacement of the bead from the centre of the trap can be used to more accurately define the forces applied by growing germ tube tips. Further, more accurate, calibration of the relationship between force and bead displacement, will be necessary for future studies. Also, it will be necessary to determine the force per unit area experienced by the germ tube tip. This will require high resolution imaging of the contact area (e.g. by using fluorescence microscopy, or low-temperature scanning electron microscopy, Read and Jeffree, 1991; Read, 1991).

The trapping force of tweezers is weaker in the $z$ axis and because the contact is initially between two near-spherical objects it is to be expected that beads will, at times, take the path of least resistance and 'roll' off the tip in the $z$ direction. Ideally one would like the tip to push against a flat surface but trapping a flat sided object which can be orientated appropriately with the tweezers would be difficult to achieve. Using the larger beads (e.g. up to $10 \mu \mathrm{m}$ in diameter) provides a partial solution by presenting flatter surfaces to growing tips.

When germ tubes made contact with a trapped bead they exhibited a swelling growth response. Fungal hyphae have been previously found to show a range of responses to physical mechanostimuli (Read et al., 1992). These include asymmetrical cell growth or spore germination in response to contact with a surface (Kwon et al., 1991; Read et al., 1992; Kuo and Hoch, 1996), directional growth and infection structure differentiation in response to microtopographical signals (Hoch et al., 1987; Read et al., 1992; Gow, 1994; Gow et al., 1994; Read et al., 1997), multicellular infection plaque differentiation in response to compression (Lucas, 2004), and intracellular calcium changes in response to shaking (Nelson et al., 2004; Bencina et al., 2005). How germ tubes sense and respond to trapped beads is not known. However, it has recently found that mechanical perturbation (by shaking in liquid culture) of germ tubes often causes them to exhibit a swelling response and this is preceded by a transient increase in cytosolic free calcium (Marris, P.I., Hickey, P.C. \& Read, N.D., unpublished results). This suggests that the mechanostimulus may be transduced intracellularly by calcium signalling. Optically trapped beads present a novel system for administering a localised mechanostimulus for single cell studies.

The localized delivery of controlled doses of specific chemicals to different cellular regions is another way to use optically trapped beads. In this study it was demonstrated how this could be performed by using porous silica beads soaked in the actin polymerization inhibitor, latrunculin-B (Spector et al., 1983). As a result of this treatment, the extension rate of 
hyphae was inhibited and they underwent significant swelling in contrast to hyphae that were only $\sim 300 \mu \mathrm{m}$ away that continued to grow normally. In the future, this could be a very powerful method to deliver chemicals to localized regions of fungal cells when used in combination with live-cell imaging. In the present study this technique was used rather crudely and would benefit from some further improvement and refinement.

\subsection{Summary}

This chapter demonstrates the following applications of using optically trapped beads:

- Optically trapped $4 \mu \mathrm{m}$ beads can be used to provide obstructions to germ tubes and cause them to undergo swelling and cease elongation

- Leading vegetative hyphae can be mechanostimulated by repeated hitting them with trapped beads to which they seem to respond by often exhibiting a small redirection in growth

- Porous beads can be used to deliver chemicals to localized regions of hyphae

- The trapping force on a polystyrene bead can be easily calibrated using the 'escape force' method

- Leading vegetative hyphae generate a greater growth force than germ tubes

\subsection{References}

Ashkin, A., Dziedzic, J.M., Bjorkholm, J.E., Chu, S., 1986. Observation of a single-beam gradient force optical trap for dielectric particles. Opt. Lett. 11, 288-290.

Baumann, C.G., Bloomfield, V.A., Smith, S.B., Bustamante, C., Wang, M.D., Block, S.M., 2000. Stretching of single collapsed DNA molecules. Biophys. J. 78, 1965-1978.

Bencina, M., Legisa, M., Read, N.D., 2005. Cross-talk between cAMP and calcium signalling in Aspergillus niger. Mol. Microbiol. 56, 268-281.

Bracker, C.E., Murphy, D.J., Lopez-Franco, R., 1997. Laser microbeam manipulation of cell morphogenesis in growing fungal hyphae. Proc. SPIE 2983, 67-80.

Cluzel, P., Lebrun, A., Heller, C., Lavery, R., Viovy, J.L., Chatenay, D., Caron, F., 1996. DNA: an extensible molecule. Science 271, 792-794.

Gittes, F., Schmidt, C.F., 1998. Interference model for back-focal-plane displacement detection in optical tweezers. Optics Letters 23, 7-9.

Gow, N.A., 1994. Growth and guidance of the fungal hypha. Microbiology 140, 3193-3205. 
Gow, N.A., Perera, T.H., Sherwood-Higham, J., Gooday, G.W., Gregory, D.W., Marshall, D., 1994. Investigation of touch-sensitive responses by hyphae of the human pathogenic fungis Candida albicans. Scanning Microsc. 8, 705-710.

Greulich, K.O., 2005. Single-molecule studies on DNA and RNA. Chemphyschem. 6, 2458-2471.

Hoch, H.C., Staples, R.C., Whitehead, B., Comeau, J., Wolf, E.D., 1987. Signaling for growth orientation and cell differentiation by surface topography in Uromyces. Science 235, 1659-1662.

Kuo, K., Hoch, H.C., 1996. Germination of Phyllosticta ampelicida pycnidiospores: prerequisite of adhesion to the substratum and the relationship of substratum wettability. Fungal Genet. Biol. 20, 1829.

Kuo, S.C., 2001. Using optics to measure biological forces and mechanics. Traffic 2, 757-763.

Kwon, Y.H., Hoch, H.C., Aist, J.R., 1991. Initiation of appresorium formation in Uromyces appendiculatus: organization of the apex, and the responses involving microtubules and apical vesicles. Can. J. Bot. 69, 2560-2573.

Lucas, J.A., 2004. Survival, surfaces and susceptibility - the sensory biology of pathogens. Plant Pathol. 53, 679-691.

Money, N.P., 2001. Biomechanics of invasive hyphal growth. In: Howard, R.J., Gow, N.A.R. (Eds.), The Mycota, Volume III, Biology of the Fungal Cell Springer-Verlag, Berlin, Heidelberg, New York, pp. 3-17.

Money, N.P., Davis, C.M., Ravishankar, J.P., 2004. Biomechanical evidence for convergent evolution of the invasive growth process among fungi and oomycete water molds. Fungal. Genet. Biol. 41, 872876.

Nelson, G., Kozlova-Zwinderman, O., Collis, A.J., Knight, M.R., Fincham, J.R., Stanger, C.P., Renwick, A., Hessing, J.G., Punt, P.J., van den Hondel, C.A., Read, N.D., 2004. Calcium measurement in living filamentous fungi expressing codon-optimized aequorin. Mol. Microbiol. 52, $1437-1450$.

Read, N.D., 1991. Low-temperature scanning electron microscopy of fungi and fungus-plant interactions. In: Mendgen, K., Lesemann, D.E. (Eds.), Electron Microscopy of Plant Pathogens Springer-Verlag, Berlin, pp. 17-29.

Read, N.D., Jeffree, C.E., 1991. Low-temperature scanning electron microscopy in biology. J. Microsc. 161 ( Pt 1), 59-72.

Read, N.D., Kellock, L.J., Collins, T., Grundlach, A.M., 1997. Role of touch sensing for infection structure differentiation in cereal rust fungi. Planta 202, 163-170.

Read, N.D., Kellock, L.J., Knight, H., Trewavas, A.J., 1992. Contact sensing during infection by fungal pathogens. In: Callow, J.A., Green, J.R. (Eds.), Perspectives in Plant Cell Recognition Cambridge University Press, Cambridge, pp. 137-172.

Sheetz, M.P., 1998. Laser Tweezers in Cell Biology. Meth. Cell Biol. 55.

Spector, I., Shochet, N.R., Kashman, Y., Groweiss, A., 1983. Latrunculins: novel marine toxins that disrupt microfilament organization in cultured cells. Science 219, 493-495.

Wilson, L., Besseling, R., Arlt, J., Poon, W.C.K., 2005. Force measurement in colloidal glasses using optical tweezers. Proc. SPIE 5930, 1-8. 
Wright, G.D., Arlt, J., Poon, W.C., Read, N.D., 2007. Optical tweezer micromanipulation of filamentous fungi. Fungal. Genet. Biol. 44, 1-13.

Wright, G.D., Arlt, J., Poon, W.C.K., Read, N.D., 2005. Measuring fungal forces with optical tweezers. Proc. SPIE 5930, F1-F7. 


\section{Chapter 6 - Overall summary and future work}

\subsection{Biological research}

The aim of this chapter is to provide an overview of the research presented in this thesis and propose future work which leads on from it. Whilst my research has been very technique focussed, it opens the way for fungal biologists, armed with an optical tweezer system, to undertake a wide range of novel experimental research.

Results presented in Chapter 3 demonstrated that conidia and conidial germlings can be easily optically trapped, manipulated and moved using relatively low laser powers of a $785 \mathrm{~nm}$ diode laser and that by adding a second trapping laser into the system more precise manipulation of the cells was possible. Combining this with developments in sample preparation, and evidence that cells could be trapped for moderate lengths of time without perturbation, facilitated the improvement of the CAT homing assay (Fleißner et al., 2005; Roca et al., 2005). Now routine, this assay will prove useful in the investigation of the cell-cell signalling between homing CATs, and particularly to screen deletion mutants compromised in signalling to determine which are defective in CAT homing. The combination of the CAT homing assay with imaging of the oscillatory recruitment of the MAP kinase protein, MAK-2, to CAT tips should also provide important insights into the underlying signalling mechanisms involved in CAT homing. Further upgrades to the optical tweezer system are in progress to allow such experiments to become possible, as discussed below in section 6.2 . The ability to produce stable microarrays of cells will also allow experiments into the signalling between neighbouring germinating spores. Not only will this be useful in studies on CAT induction, homing and fusion, it will also be important in studies on the autotropic germ tube avoidance response (Read, 2007). The combination of HOTs, or other multiple trap techniques (e.g. time multiplexing), with Cygel would allow for the production of, not only 2D, but 3D microarrays of cells to be established.

It has been shown that cells can be selectively stimulated, or treated, with various methods from microfluidic flow cells (Chapter 3) to using trapped beads as 'optical hammers' or delivery vehicles for locally applied chemical doses (Chapter 5). There is immense scope to refine these techniques. For example, for chemical delivery it would be highly desirable to use impermeant capsules to house the chemical until release of their contents is triggered 
(e.g. by laser photolysis, as demonstrated by Sun and Chiu, 2004). Alternatively beads coated with chemicals, such as pheromones, could be pressed against cells to activate ligandreceptor responses (as shown by Wei et al., 1999). The combination of the optical tweezers with various advanced fluorescence imaging techniques (e.g. 4D image deconvolution, Parton and Davis, 2005), could allow improved visualization of cellular responses, particularly when used in conjunction with the labelling of proteins with GFP and its derivatives. Imaging the cytosolic free calcium levels could also help elucidate how stimuli, such as the mechanical stimulation of hyphal tips, are transduced within the cell. Combining optical tweezers with Raman spectroscopy (Ajito and Torimitsu, 2001; Xie et al., 2002; Tang et al., 2005) could also be used for the simultaneous spectroscopic analysis and trapping of cells to analyse changes in the chemical make-up of the internal cell environment, including changes in their chemistry which may result from possible photodamage during trapping (Ramser et al., 2004).

The $785 \mathrm{~nm}$ optical trap used for the majority of experiments reported in this thesis has been shown not to damage cells with short to moderate durations of exposure, even at full power, but long durations can prevent the germination of conidia. It is clear from the literature that certain wavelengths of light are best avoided for optical tweezer experiments, and that others are relatively safe to use, depending somewhat on the sample under investigation. Using a tuneable trapping laser (e.g. a titanium-sapphire laser; Liang et al., 1996; Neuman et al., 1999; Leitz et al., 2002) future experiments could be performed to investigate the optimal wavelength of light, and the maximum duration of laser exposure different types of fungal cells (e.g. conidia, germ tubes and leading hypha) can tolerate.

Results reported in Chapter 4 demonstrated that organelles within cells can be manipulated, to varying extents, using optical tweezers. Woronin bodies, and other organelles of high refractive index relative to the cytosol, can be trapped and their position manipulated, unless they are tethered. Vacuoles, and other organelles of low refractive index relative to the cytosol, are repelled by the laser and their position within the cell manipulated by a process akin to repelling magnets. Ectopically produced crystals have been shown to have potential as 'handles' within cells to manipulate organelles that cannot otherwise be trapped. The fact that the nucleus of Schizosacc. pombe cells could not be moved using this technique presents some interesting future experiments to elucidate which cytoskeletal elements are responsible for the establishment and maintenance of nuclear position. Performing these experiments in the presence of pharmacological inhibitors, that disrupt microtubules or actin microfilaments, would be useful here. 
The Spitzenkörper presented a very interesting exception to other results involving the manipulation of organelles. In theory, due to the refractive properties of the Spitzenkörper, one would have expected it to be trapped with optical tweezers. However as was show here, and previously (Bracker et al., 1997), optical tweezers can be used to control the direction of growth by manipulating the position of the Spitzenkörper within hyphal tips by apparently repelling it. Future work should test whether the response is a purely physical phenomenon (e.g. optical repulsion of the Spitzenkörper), or a photoreceptor mediated negative phototropism. Repeating experiments with a range of wavelengths of light using strains in which the genes encoding different photoreceptors (Borkovich et al., 2004) have been mutated, and attempting to image the behaviour of the Spitzenkörper during redirection could be performed. There is great diversity in the morphology and optical properties of Spitzenkörper between different filamentous fungi (Lopez-Franco and Bracker, 1996), so analyses of the reaction of Spitzenkörper of different species to optical trapping may prove enlightening. It was also shown in Chapter 4 that light patterns generated with HOTs could achieve continuous redirection over extended ranges. Pseudowalls generated by HOTs were shown to be much more efficient for hyphal growth redirection, due to the lower intensity of light and greater axial extent of the light pattern. To increase the tolerance still further, the possibility of creating true 3D walls of light (Whyte and Courtial, 2005) should be explored in future work. It would also be desirable to build a HOT system using a less damaging wavelength of light, preferably in the near infrared part of the light spectrum. The extended range redirection technique could be used to force hyphal tips into close proximity and assess the autotropic avoidance response or induction of vegetative hyphal fusion.

Results in Chapter 5 showed that optically trapped $4 \mu \mathrm{m}$ beads can be used to provide obstructions to germ tubes and cause them to undergo swelling and cease elongation. As the trapping force on a polystyrene bead for given laser powers and spring constant has been calibrated using the 'escape force' method it should be possible, in future experiments, to pin down the force that germ tubes of different sizes and stages of development can produce. For comparison with previous work (Money et al., 2004) it will be necessary to calculate or measure the contact area between a germ tube tip and bead. High resolution fluorescence imaging or low-temperature scanning electron microscopy (Read and Jeffree, 1991; Read, 1991) would be helpful here. The growth forces could then be expressed as force per unit area, or applied pressures $\left(\mu \mathrm{N}_{\mu \mathrm{m}^{-2}}\right)$. Depending on the requirements of sample preparation it may be possible to use a dedicated force measurement optical tweezer system (using a QPD 
like that described in Gittes and Schmidt, 1998; Wilson et al., 2005) to make more precise measurements in the future.

\subsection{Future developments to the optical tweezers}

The next generation $785 \mathrm{~nm}$ optical tweezer system is currently in production at the University of Edinburgh for some of the future work suggested here to be performed. In Chapters 3-5 various potential system upgrades have been mentioned that could enhance particular experiments. The most important of these is to improve the widefield fluorescence excitation illumination light source. The recent advancements in this area of microscopy hardware has seen a move away from the traditional arc lamps towards the use of LEDs, which produce much less heat, specific bandwidths of light, and can be turned on and off quickly. Combining this illumination source with a computer controlled shutter system would greatly reduce the photobleaching of fluorescent cells and permit the completion of the envisaged MAK-2-GFP experiments described in section 3.2.1.2. Advanced imaging techniques (e.g. FRET, FLIM or CATs) in combination with optical tweezers would allow for numerous novel experiments to be completed. Whilst the computer controlled galvanometric mirrors permit the precise position and movement of the trapping position, long distance transport of cells across the coverglass (e.g. when establishing microarrays of cells with the PVA technique; section 3.2.1.3) required careful movement of the microscope stage. In this respect it would be desirable to have a computer controlled motorised stage to reduce the likelihood of losing cells from the trap. The benefits of multiple traps are clear for a number of experiments. Multiple trap systems, despite their increased complexity, are becoming increasingly common. An upgrade to the current system's software could produce multiple trapping sites by time-multiplexing the trap position using the galvanometric mirrors (Mio et al., 2000). It would be quite easy, although expensive, to replace the $785 \mathrm{~nm}$ diode laser with a tuneable laser source (e.g. titanium-sapphire laser) or indeed a system for inputting any laser for the desired application. Such versatility could allow for the use of laser scissors or a greater choice of wavelengths to reduce the potential photodamage to cells.

\subsection{References}

Ajito, K., Torimitsu, K., 2001. Near-infrared Raman spectroscopy of single particles. Trac-Trends in Analytical Chemistry 20, 255-262.

Borkovich, K.A., Alex, L.A., Yarden, O., Freitag, M., Turner, G.E., Read, N.D., Seiler, S., BellPedersen, D., Paietta, J., Plesofsky, N., Plamann, M., Goodrich-Tanrikulu, M., Schulte, U., 
Mannhaupt, G., Nargang, F.E., Radford, A., Selitrennikoff, C., Galagan, J.E., Dunlap, J.C., Loros, J.J., Catcheside, D., Inoue, H., Aramayo, R., Polymenis, M., Selker, E.U., Sachs, M.S., Marzluf, G.A., Paulsen, I., Davis, R., Ebbole, D.J., Zelter, A., Kalkman, E.R., O'Rourke, R., Bowring, F., Yeadon, J., Ishii, C., Suzuki, K., Sakai, W., Pratt, R., 2004. Lessons from the genome sequence of Neurospora crassa: tracing the path from genomic blueprint to multicellular organism. Microbiol. Mol. Biol. Rev. 68, 1-108.

Bracker, C.E., Murphy, D.J., Lopez-Franco, R., 1997. Laser microbeam manipulation of cell morphogenesis in growing fungal hyphae. Proc. SPIE 2983, 67-80.

Fleißner, A., Sarkar, S., Jacobson, D.J., Roca, M.G., Read, N.D., Glass, N.L., 2005. The so locus is required for vegetative cell fusion and postfertilization events in Neurospora crassa. Eukaryot. Cell 4, 920-930.

Gittes, F., Schmidt, C.F., 1998. Interference model for back-focal-plane displacement detection in optical tweezers. Optics Letters 23, 7-9.

Leitz, G., Fallman, E., Tuck, S., Axner, O., 2002. Stress response in Caenorhabditis elegans caused by optical tweezers: Wavelength, power, and time dependence. Biophys. J. 82, 2224-2231.

Liang, H., Vu, K.T., Krishnan, P., Trang, T.C., Shin, D., Kimel, S., Berns, M.W., 1996. Wavelength dependence of cell cloning efficiency after optical trapping. Biophys. J. 70, 1529-1533.

Lopez-Franco, R., Bracker, C.E., 1996. Diversity and dynamics of the Spitzenkörper in growing hyphal tips of higher fungi. Protoplasma 195, 90-111.

Mio, C., Gong, T., Terray, A., Marr, D.W.M., 2000. Design of a scanning laser optical trap for multiparticle manipulation. Rev. Sci. Instrum. 71, 2196-2200.

Money, N.P., Davis, C.M., Ravishankar, J.P., 2004. Biomechanical evidence for convergent evolution of the invasive growth process among fungi and oomycete water molds. Fungal. Genet. Biol. 41, 872876.

Neuman, K.C., Chadd, E.H., Liou, G.F., Bergman, K., Block, S.M., 1999. Characterization of photodamage to Escherichia coli in optical traps. Biophys. J. 77, 2856-2863.

Parton, R.M., Davis, I., 2005. Lifting the fog: image restoration by deconvolution. In: Celis, J.E. (Ed.), Cell Biology, A Laboratory Handbook. Academic Press, London, pp. 187-200.

Ramser, K., Logg, K., Goksor, M., Enger, J., Kall, M., Hanstorp, D., 2004. Resonance Raman spectroscopy of optically trapped functional erythrocytes. J. Biomed. Opt. 9, 593-600.

Read, N.D., 1991. Low-temperature scanning electron microscopy of fungi and fungus-plant interactions. In: Mendgen, K., Lesemann, D.E. (Eds.), Electron Microscopy of Plant Pathogens Springer-Verlag, Berlin, pp. 17-29.

Read, N.D., 2007. Environmental sensing and the filamentous fungal lifestyle. In: Gadd, G.M., Watkinson, S.C., Dyer, P. (Eds.), Fungi in the Environment Cambridge University Press, Cambridge, pp. 38-57.

Read, N.D., Jeffree, C.E., 1991. Low-temperature scanning electron microscopy in biology. J. Microsc. 161 ( Pt 1), 59-72.

Roca, M.G., Arlt, J., Jeffree, C.E., Read, N.D., 2005. Cell biology of conidial anastomosis tubes in Neurospora crassa. Eukaryot. Cell 4, 911-919. 
Sun, B., Chiu, D.T., 2004. Synthesis, loading, and application of individual nanocapsules for probing single-cell signaling. Langmuir 20, 4614-4620.

Tang, W., Newton, R.J., Xie, C.A., Li, Y.Q., Whitley, N., 2005. Non-destructive analysis of the nuclei of transgenic living cells using laser tweezers and near-infrared raman spectroscopic technique. Genomics Proteomics. Bioinformatics. 3, 169-178.

Wei, X., Tromberg, B.J., Cahalan, M.D., 1999. Mapping the sensitivity of T cells with an optical trap: Polarity and minimal number of receptors for $\mathrm{Ca}^{2+}$ signaling. Proc. Natl. Acad. Sci. U. S. A 96, 84718476.

Whyte, G., Courtial, J., 2005. Experimental demonstration of holographic three-dimensional light shaping using a Gerchberg-Saxton algorithm. New J. Phys. 7, 117.

Wilson, L., Besseling, R., Arlt, J., Poon, W.C.K., 2005. Force measurement in colloidal glasses using optical tweezers. Proc. SPIE 5930, 1-8.

Xie, C., Dinno, M.A., Li, Y., 2002. Near-infrared Raman spectroscopy of single optically trapped biological cells. Opt. Lett. 27, 249-251. 


\section{Chapter 7 - Publications}

The following papers are bound into the back of this thesis:

Wright, G.D., Arlt, J., Poon, W.C.K., Read, N.D., 2005. Measuring fungal forces with optical tweezers. Proc. SPIE 5930, F1-F7.

Wright, G.D., Arlt, J., Poon, W.C., Read, N.D., 2007. Optical tweezer micromanipulation of filamentous fungi. Fungal. Genet. Biol. 44, 1-13.

Wright, G.D., Arlt, J., Poon, W.C.K., Read, N.D., 2007. Experimentally manipulating fungi with optical tweezers. Mycoscience 48, 15-19

Burnham, D.R., Wright, G.D., Read, N.D., McGloin, D., 2007. Holographic and single beam optical manipulation of hyphal tip growth in filamentous fungi. J. Opt. A: Pure Appl. Opt. 9, S172-S179. 


\section{Appendix 1 - Supplementary movies on CD}

Chapter 3 Manipulating whole fungal cells with optical tweezers

Chapter 4 Manipulating organelles within fungal cells with optical tweezers

Chapter 5 Manipulating microspheres with optical tweezers as experimental tools to study fungal cells

\section{Viewing instructions}

The CD should autorun when inserted into the computer or with a double-click of the CD icon. If this does not happen, browse the $\mathrm{CD}$ and open the file index.html with a web browser (e.g. Internet Explorer or Mozilla Firefox, the latter works best). Once open, click on the links to navigate through the chapters and files. For optimal performance copy the entire contents of the $\mathrm{CD}$ into a folder on your hard drive, then open index.hml. Many of the movies are also available online at http://www.fungalcell.org. 


\title{
Optical tweezer micromanipulation of filamentous fungi
}

\author{
Graham D. Wright \\ PhD Thesis \\ Appendix 1 - Supplementary movies
}

Chapter 3 - Manipulating whole fungal cells with optical tweezers

Chapter 4 - Manipulating organelles within fungal cells with optical tweezers

Chapter 5 - Manipulating microspheres with optical tweezers as experimental tools to study fungal cells

PDF of thesis and publications
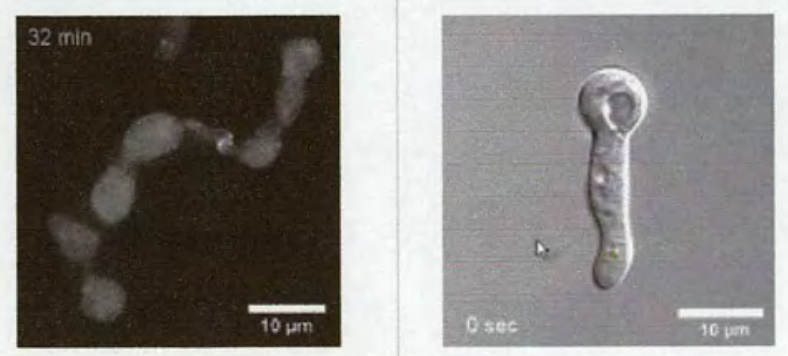

Home

$0 \sec$
Chapter 3
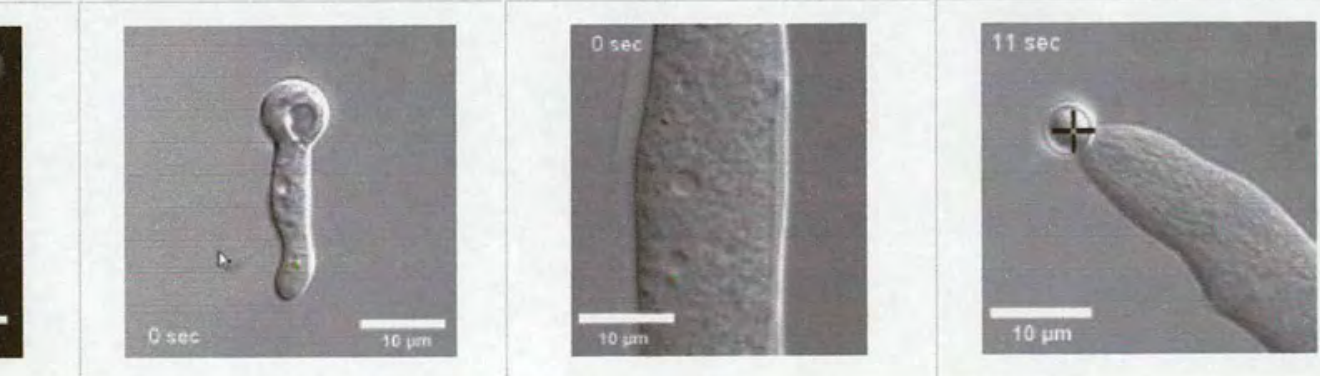

Chapter 4

Chapter 5 


\section{Appendix 1 - Supplementary movies \\ Chapter 3 - Manipulating whole fungal cells with optical tweezers}

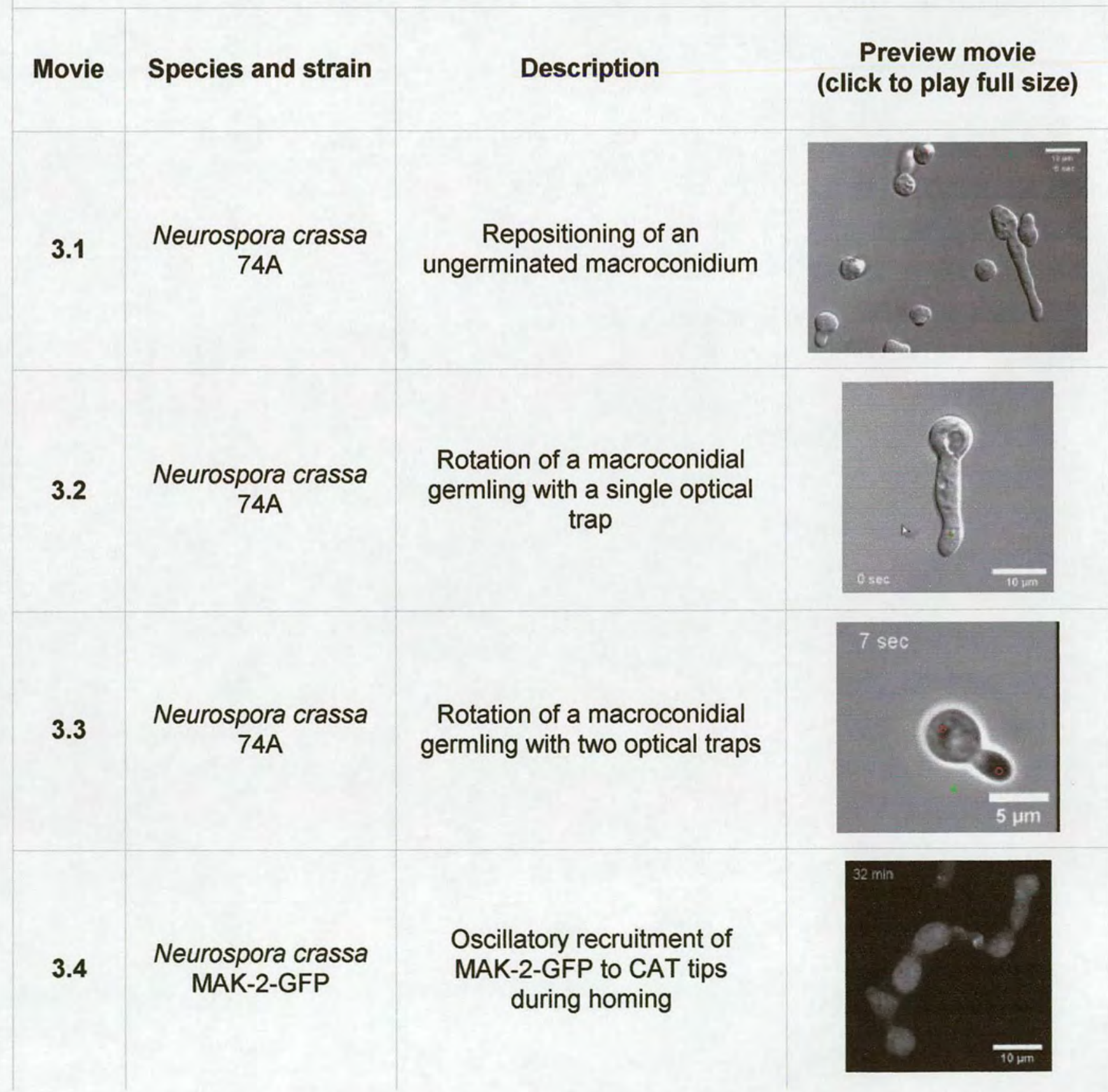





\section{Appendix 1 - Supplementary movies \\ Chapter 4 - Manipulating organelles within fungal cells with optical tweezers}

\begin{tabular}{|l|l|l|l|}
\hline Movie & Species and strain & Description & $\begin{array}{c}\text { Preview movie } \\
\text { (click to play full size) }\end{array}$ \\
\hline 4.1 & $\begin{array}{c}\text { Neurospora crassa } \\
74 \mathrm{~A}\end{array}$ & $\begin{array}{c}\text { Trapping and moving a } \\
\text { Woronin body within a hypha }\end{array}$ \\
\hline 4.2 & $\begin{array}{c}\text { Aspergillus nidulans } \\
\text { HexA-GFP }\end{array}$ & $\begin{array}{c}\text { Inability to move Woronin } \\
\text { bodies tethered to the septa }\end{array}$ \\
\hline 4.3 & $\begin{array}{c}\text { Schizosaccharomyces } \\
\text { pombe } \\
\text { Polyhedrin crystals }\end{array}$ & $\begin{array}{c}\text { Manipulating ectopic crystals of } \\
\text { polyhedrin inside the nucleus }\end{array}$ \\
\hline
\end{tabular}




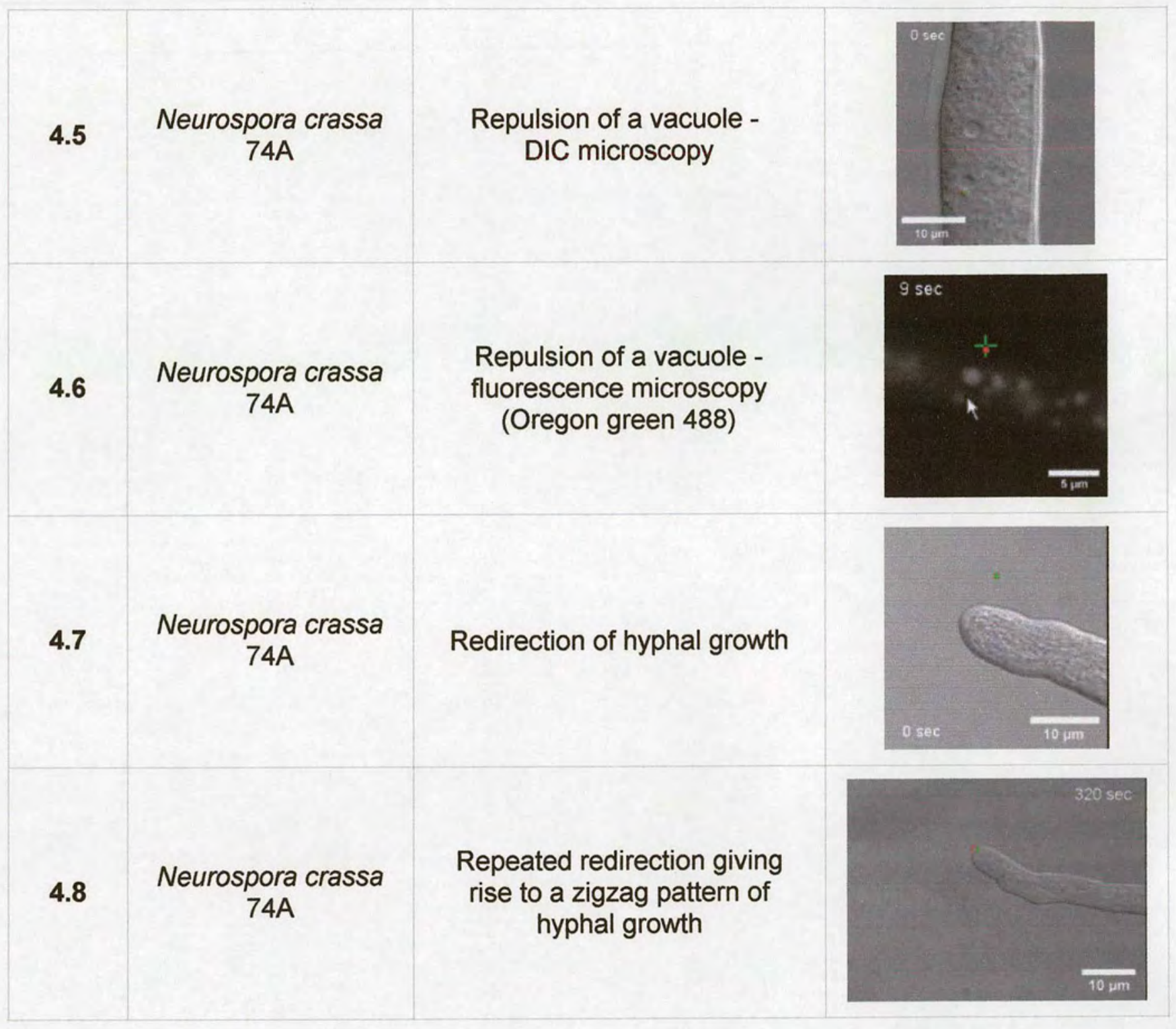

Home $\quad$ Chapter $3 \quad$ Chapter $4 \quad$ Chapter 5

PDF of thesis and publications 


\section{Appendix 1 - Supplementary movies}

\section{Chapter 5 - Manipulating microspheres with optical tweezers as experimental tools to study fungal cells}

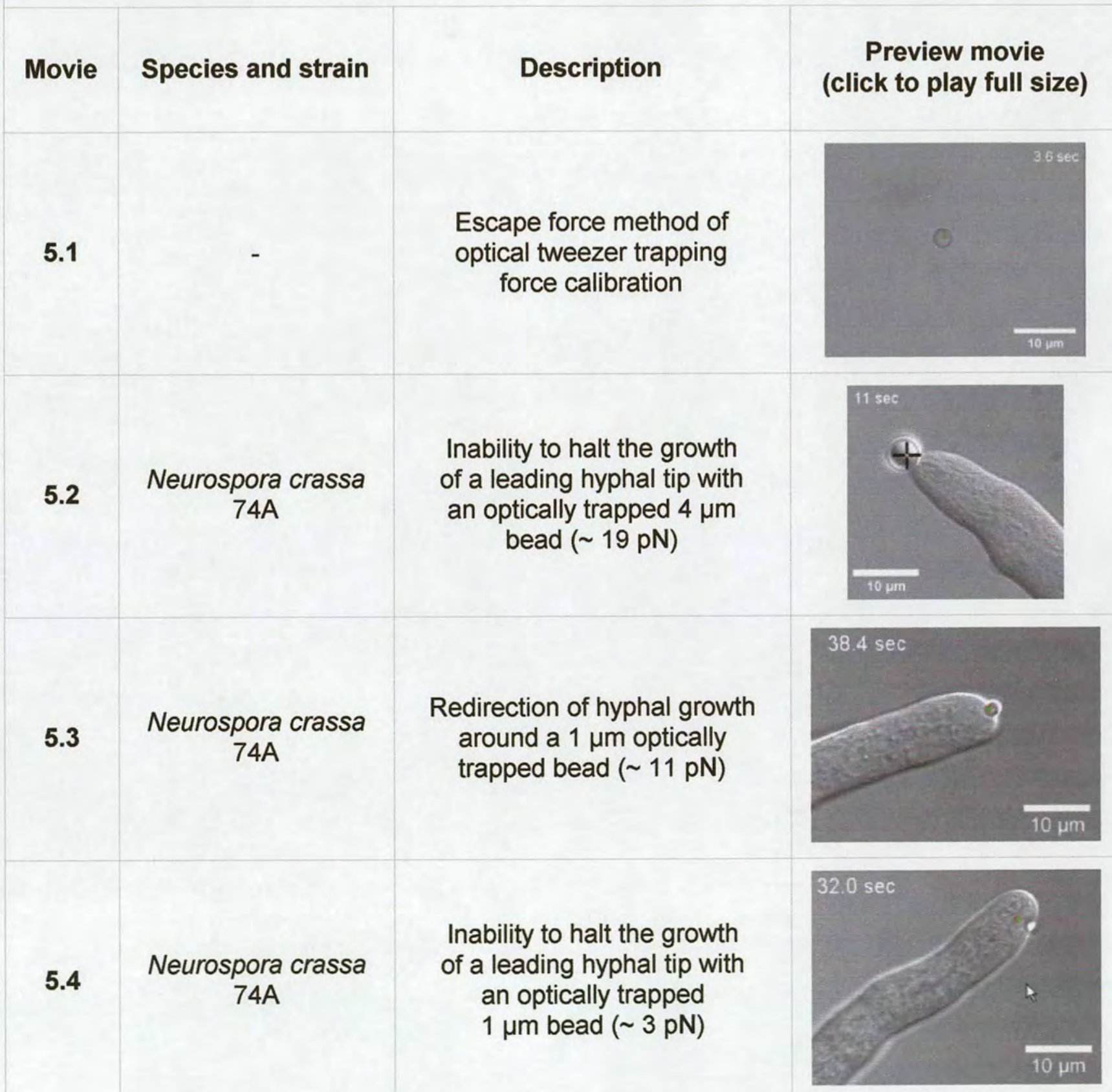




\begin{tabular}{|c|c|c|}
\hline 5.5 & Neurospora crassa & $\begin{array}{c}\text { Inhibition of germ tube } \\
\text { growth with an optically } \\
\text { trapped } 4 \mu \mathrm{m} \text { bead } \\
(\sim 19 \mathrm{pN})\end{array}$ \\
\hline 5.6 & Neurospora crassa & $\begin{array}{c}\text { Mechanostimulaton of a } \\
\text { leading hypha using a } \\
10 \mu \mathrm{Am} \text { bead as an } \\
\text { 'optical hammer' }\end{array}$ \\
\hline & & \\
\hline
\end{tabular}

Home 


\title{
Measuring Fungal Growth Forces with Optical Tweezers
}

\author{
Graham D. Wright ${ }^{* a, c}$, Jochen Arlt ${ }^{\mathrm{b}, \mathrm{c}}$, Wilson C.K. Poon ${ }^{\mathrm{b}, \mathrm{c}}$, Nick D. Read ${ }^{\mathrm{a}, \mathrm{c}}$ \\ ${ }^{a}$ Fungal Cell Biology Group, Institute of Cell Biology, \\ University of Edinburgh, Rutherford Building, Edinburgh, EH9 3JH, UK

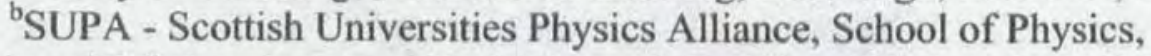 \\ University of Edinburgh, James Clerk Maxwell Building, Edinburgh, EH9 3JZ, UK \\ ${ }^{c}$ COSMIC - ollaborative Optical Spectroscopy, Micromanipulation \& Imaging Centre, \\ University of Edinburgh, James Clerk Maxwell Building, Edinburgh, EH9 3JZ, UK
}

\begin{abstract}
Optical tweezers are proving themselves useful and powerful tools in many areas of biological research, including fungal cell biology. Here we investigate their potential as a novel method for measuring the growth forces of fungal hyphae. We have calibrated the trapping forces produced by our tweezers system, and using beads have investigated whether growing hyphae can overcome this force and push beads out of the trap. Although $4 \mu \mathrm{m}$ beads were pushed out of the trap by fungal hyphae, using $1 \mu \mathrm{m}$ beads produced some more intriguing results. The problems encountered with this method of measurement and possible solutions are discussed.
\end{abstract}

Keywords: Fungi; hyphal tip growth; Neurospora crassa; optical tweezers; Spitzenkörper.

\section{INTRODUCTION}

Optical tweezers utilize the forces produced when focused laser light interacts with an object to trap it in 3-D ${ }^{14}$. Subsequent movement of the sample or the laser allows precise positional control of the trapped object. Tweezers integrated with live-cell imaging are proving to be exquisitely powerful micromanipulative tools in cell biological studies $^{2.5-7}$. In the present work we have investigated their potential for use to measure the growth forces generated by filamentous fungi.

Filamentous fungi are more closely related to animals than plants and grow in a very unique manner producing a complex interconnected network of multinucleate, tubular cellular elements called hyphae. These hyphae grow at their tips and penetrate with force, and by digestion, through the microenvironments which they inhabit. Neurospora crassa is commonly used as a model filamentous fungus for experimental studies ${ }^{8,9}$, and was the first filamentous fungus to have its complete genome sequenced ${ }^{10.11}$. Advantages of $N$. crassa for use in optical tweezers studies ${ }^{12}$ include: (1) it is easily cultured in the laboratory, (2) it grows rapidly at rates of up to $2 \mu \mathrm{m}$ per second ${ }^{13},(3)$ it produces large hyphae (up to $15 \mu \mathrm{m}$ in width), which are well suited for live-cell imaging ${ }^{14-16},(4)$ a vast collection of strains and mutants are available (www.fgsc.net), and (5) it is very amenable to molecular biology studies (e.g. tagging proteins with green fluorescent protein $\left.^{14}\right)$.

Despite these advantages, few papers have been published in which optical tweezers have been used to micromanipulate living cells of filamentous fungi ${ }^{12.13,17.18}$. Berns et al ${ }^{17}$ worked with Nectria haematococca and were able to manipulate different organelles within hyphae without obvious damage. They found the order of organelle responsiveness to trapping to be: lipid bodies $>$ nucleoli $>$ mitochondria $>$ chromosomes. Bracker et al ${ }^{13}$ reported successful attempts of manipulating the Spitzenkörper in Trichoderma viride and Sclerotinia sclerotionum (the Spitzenkörper is a complex organelle assemblage within hyphal tips which is intimately associated with hyphal tip growth and morphogenesis ${ }^{19}$, Fig. 1). Using optical tweezers, they showed that altering the position of the Spitzenkörper changed the direction of hyphal growth, and that concentrating secretory vesicles could be used to initiate branching in abnormal hyphal

graham@fungalcell.org; phone +44 (0) 131651 3337; web www.cosmic.ed.ac.uk, www.fungalcell.org 
locations. Roca $e \mathrm{al}^{18}$ developed an optical tweezers homing assay to investigate signaling between two germlings and characterize conidial anastomosis tubes (CATs) in $N$. crassa. We have also provided an overview of how tweezers can be used as powerful experimental tools in fungal cell biology research ${ }^{12}$.

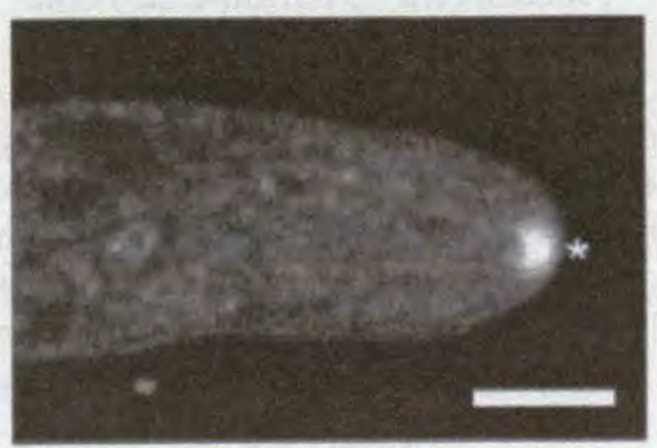

Figure 1: Concentration of secretory vesicles within the Spitzenkörper $\left({ }^{*}\right)$ of a growing hyphal tip of Neurospora crassa after labelling with the membrane-selective dye, FM4-64 ${ }^{16}$. Bar $=5 \mu \mathrm{m}$.

The vast majority of the work on fungal forces has been performed by Nik Money and collaborators ${ }^{20.21}$. His group was the first to document force measurements in vegetative hyphae ${ }^{22}$. Using a miniaturised strain gauge they measured the forces produced by hyphal tips and subsequently calculated the applied pressure (force per unit area, $\mu \mathrm{N} \mu \mathrm{m}^{-2}$ or MPa). Pressure ranging from $0.04 \mathrm{MPa}$ (in Sordaria fimicola) to $0.09 \mathrm{MPa}$ (in N. crassa) were reported. Penetration by a fungus through its natural environment is also usually facilitated by the fungus secreting a cocktail of digestive enzymes. In fungal plant pathogens, hyphae often develop into specialized infection structures (e.g. on a leaf or root surface) which can generate extremely large forces (turgor pressures of $80 \mathrm{MPa}$ have been measured ${ }^{23.24}$ ) allowing these fungi to efficiently breach the tough host defences. The main contributors to the growth forces of fungal hyphae are believed to be cell turgor and the actin cytoskeleton ${ }^{20.21 .25}$. To date there have been no reports of using optical tweezers to measure fungal growth forces.

The aims of the present paper are to: 1) describe the optical tweezers system which we have used to attempt force measurement of fungal cells, 2) report on the calibration of the trapping forces produced by our optical tweezers system, 3) present our preliminary results on measuring fungal growth forces, and finally 4) discuss the problems encountered and how these might be overcome.

\section{MATERIALS AND METHODS}

\subsection{Fungal strains and culture conditions}

The $N$. crassa wild-type strain 74-OR23-1VA ( $\# 2489$, FGSC, Kansas City, KS, USA) was used, and was grown on Vogel's medium ${ }^{8}$ solidified with agar in Petri dishes. Hyphal tips for experimental analysis were produced after incubating cultures for $\sim 24 \mathrm{~h}$ at $25^{\circ} \mathrm{C}$.

\subsection{Sample Preparation}

Agar blocks (ca. $2 \times 2 \times 0.5 \mathrm{~cm}$ ) bearing growing hypha were excised from the colony periphery. Each block was inverted onto a droplet of liquid Vogel's medium on a $48 \times 64 \mathrm{~mm}$ glass coverslip (R.A. Lamb, Surrey, UK) ${ }^{16,26}$ containing polystyrene beads with nominal diameters of $1 \mu \mathrm{m}$ or $4 \mu \mathrm{m}$ (Interfacial Dynamics Corp., Portland, OR, USA); the actual diameters of the beads were measured by microscopy to be $1.7 \mu \mathrm{m}$ and $4.7 \mu \mathrm{m}$ respectively. The liquid medium was periodically refreshed to prevent the agar drying out or oxygen being depleted. The agar block was supported on strips of adhesive electrician's insulation tape to help maintain a liquid layer and prevent the beads from adhering to the agar. Poly-L-Lysine (Sigma, Poole, Dorset, UK) was used to coat the coverslips to aid adhesion of the cells to the glass. Beads were soaked in a $1 \%(\mathrm{w} / \mathrm{v})$ solution of bovine serum albumin to prevent adhesion to cells, coverslips or agar. The same preparation technique was used for confocal microscopy, but omitting the insulation tape, beads and poly-L-lysine ${ }^{16,26}$. The Spitzenkörper was stained in growing hyphal tips by incorporating $15 \mu \mathrm{M}$ FM4-64 (http://probes.invitrogen.com/) in the droplet of Vogel's medium. 


\subsection{Microscopy}

Microscopy was performed with a Nikon Eclipse TE2000-U inverted microscope into which the tweezers set up had been integrated. A Nikon Plan Apo 100x 1.4 N.A. DIC H oil immersion objective was used with bright field or differential interference contrast (DIC) optics. To increase the camera's field of view a $0.7 \times$ demagnifying C-mount was used when necessary. Confocal microscopy was performed using a Bio-Rad Radiance 2100 system equipped with an argon ion laser, and mounted on a Nikon TE2000-U inverted microscope. FM4-64 was imaged after excitation with the $488 \mathrm{~nm}$ laser line and fluorescence detected at $>600 \mathrm{~nm}$. A Nikon Plan Apo $60 \times 1.2$ N.A. water immersion objective was used for imaging.

\subsection{Optical tweezers system}

A simple optical tweezers system has been designed and built in-house and integrated into a Nikon Eclipse TE2000-U inverted microscope (Fig. 2). A near-infrared diode laser $(\lambda=785 \mathrm{~nm})$, adjustable up to an output of $70 \mathrm{~mW}, 58.3 \mathrm{~mW}$ at the objective back aperture, (VPSL-0785-070-x-5-A from Blue Sky Research, Milpitas, CA, USA), directed towards 2 galvanometer beam steering mirrors is redirected into the rear of the microscope. A slight modification made to the microscope, which involved lifting the nosepiece, allows the optical tweezers to be used simultaneously with fluorescence microscopy. A dichroic mirror then reflects the beam into the objective lens. A computer program written in LabVIEW 6.1 (National Instruments, Austin, TX, USA) allows the user to move the trap across the camera (Applied Vision Technologies, Dolphin 145-F from FirstSight Vision, Surrey, UK) field of view by a mouse-click at the desired destination. Calibration was completed using the escape force method and applying Stoke's law ${ }^{7.27}$.
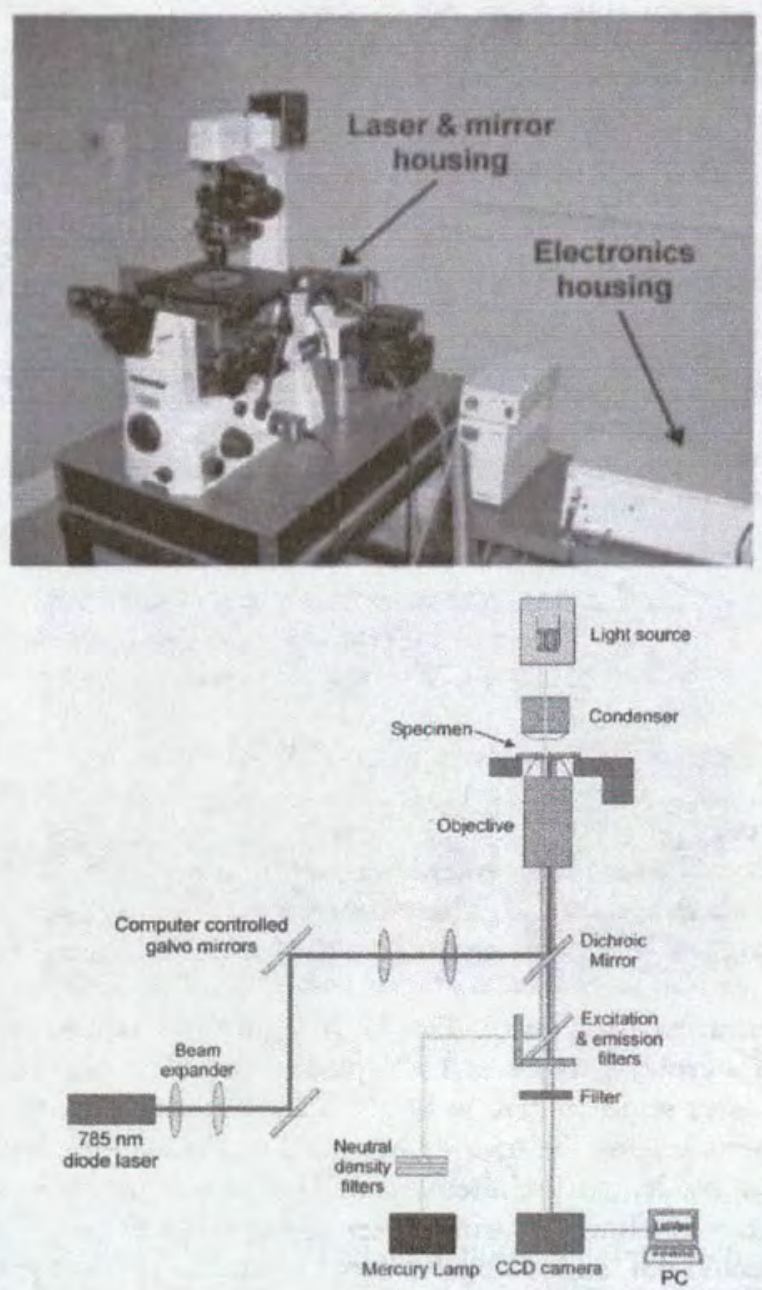

Figure 2: Photograph and schematic layout of the optical tweezers system. 


\subsection{Digital image processing and animation}

Images of hyphae being subjected to optical tweezing were captured either singularly through the control software directly from the camera, or as a time course using screen capture software (HyperCam, www.hyperionics.com). Confocal images were captured using Lasersharp software (version 5.2; Bio-Rad) and were initially viewed using Confocal Assistant software (version 4.02). Further processing for all images was carried out with Paintshop Pro (version 7; JASC). Time courses of images were edited and built up into animation movies (avi files) using Animation Shop (version 3; JASC) and ImageJ (freely available software, www.rsb.info.nih.gov/ij/).

\section{RESULTS}

\subsection{Tweezers calibration}

The escape force method ${ }^{7}$ of tweezers calibration was performed for $1 \mu \mathrm{m}$ and $4 \mu \mathrm{m}$ polystyrene beads (Fig. 3). This method involved trapping a bead and, using a motorised stage, moving its environment around it at increasing velocity until the bead escapes the trap. The trapping force, assumed to be equal to the viscous drag at the point of escape, is then calculated, for that laser power, using Stoke's law:

$$
F=6 \pi \cdot \eta \cdot a . v \text {. }
$$

Where $\eta$ is the viscosity $\left(0.001025 \mathrm{~N} \mathrm{~s} \mathrm{~m}^{-2}\right.$ for water at $\left.20^{\circ} \mathrm{C}\right)$, a is the radius of the bead, and $\mathrm{v}$ is the velocity measured. The laser powers that could be calibrated were limited by the maximum velocity of the motorised stage. The resulting calibration graph shows a good linear relationship between the laser power and the trapping force, thereby allowing extrapolation (Fig. 3). A maximum trapping force of $\sim 11 \mathrm{pN}$ for $1 \mu \mathrm{m}$ beads and $\sim 19 \mathrm{pN}$ for $4 \mu \mathrm{m}$ beads was measured.

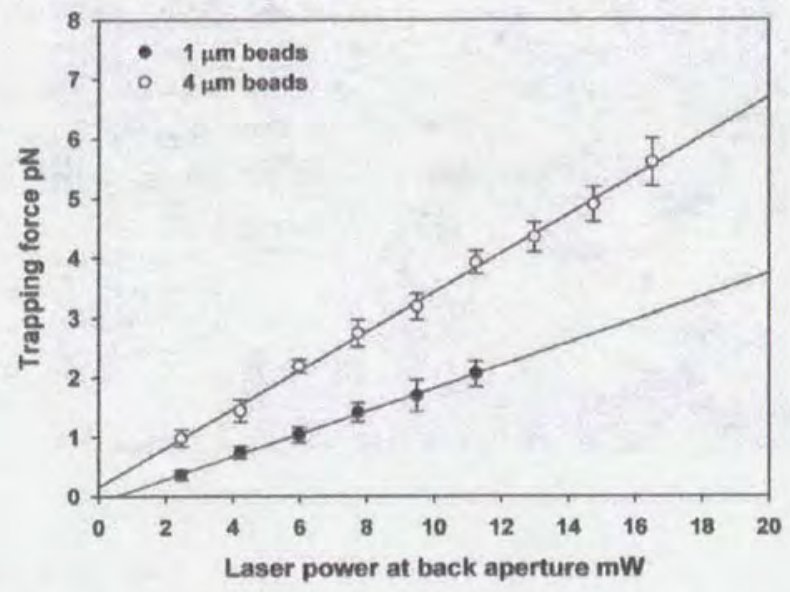

Figure 3: Calibration of the optical tweezers system.

\subsection{Measuring fungal growth forces}

Initially $4 \mu \mathrm{m}$ beads were trapped at full laser power and placed in the path of growing hyphae. Despite using full power the beads were all pushed out of the trap (Fig. 4). This was to be expected as previous reports ${ }^{22}$ have shown fungi to be able to produce forces in the $\mu \mathrm{N}$ range and the trapping force on the bead was only $\sim 19 \mathrm{pN}$.

A more interesting result was found using $1 \mu \mathrm{m}$ beads (Fig. 5). A $1 \mu \mathrm{m}$ bead trapped with the laser power set to $20 \mathrm{~mW}$ $(\sim 3 \mathrm{pN})$ was placed in the path of a growing hypha and was pushed out of the trap (Fig. 5A). However when trapping an equivalent bead with the laser power set to $70 \mathrm{~mW}(\sim 11 \mathrm{pN})$ a hypha was unable to push the bead from the trap (Fig. $5 \mathrm{~B})$. In the latter case the hypha grew around the trapped bead and continued growing. Sixteen repetitions $(\mathrm{n}=16)$ of these experiments produced similar results. At the intermediate laser power set to $39 \mathrm{~mW}(\sim 6 \mathrm{pN})$ hyphae were still able to push beads out of the trap $(\mathrm{n}=2)$. However, with a laser power set to $53 \mathrm{~mW}(\sim 8 \mathrm{pN}, \mathrm{n}=4)$ some hyphae could overcome the trap, whilst others could not, suggesting the force produced by the hyphae is roughly equivalent to the trapping force at this laser power. However this cannot be the case in the light of the results with $4 \mu \mathrm{m}$ beads. 
It is significant, and inevitable, that the hyphal tip will grow directly into the laser as it pushes on a $1 \mu \mathrm{m}$ bead. A problem associated with this is that the laser beam interferes with the Spitzenkörper by repelling it ${ }^{12.13}$ (Fig. 6). Therefore, in instances where the tip appears to grow round the trapped bed (Fig. 5B) it is actually the repositioning of the Spitzenkörper, by the laser, causing redirection of the growth.
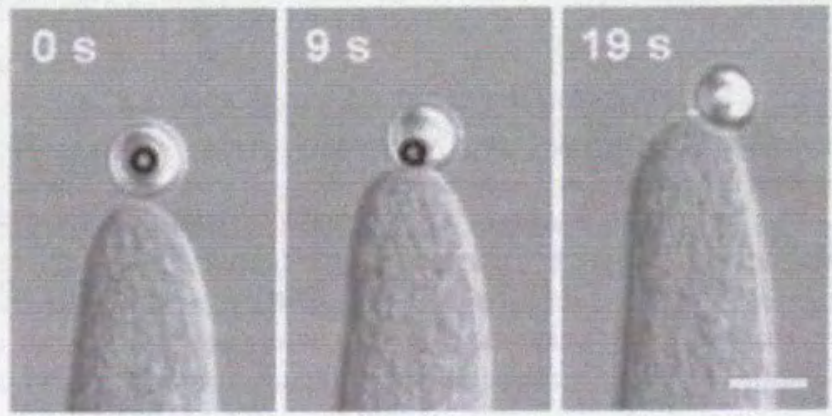

Figure 4: A time course of a growing hyphae showing that a $4 \mu \mathrm{m}$ bead, trapped at full laser power ( $70 \mathrm{~mW})$, can be pushed out of the trap. The circle indicates the position of the optical trap. Bar $=5 \mu \mathrm{m}$.
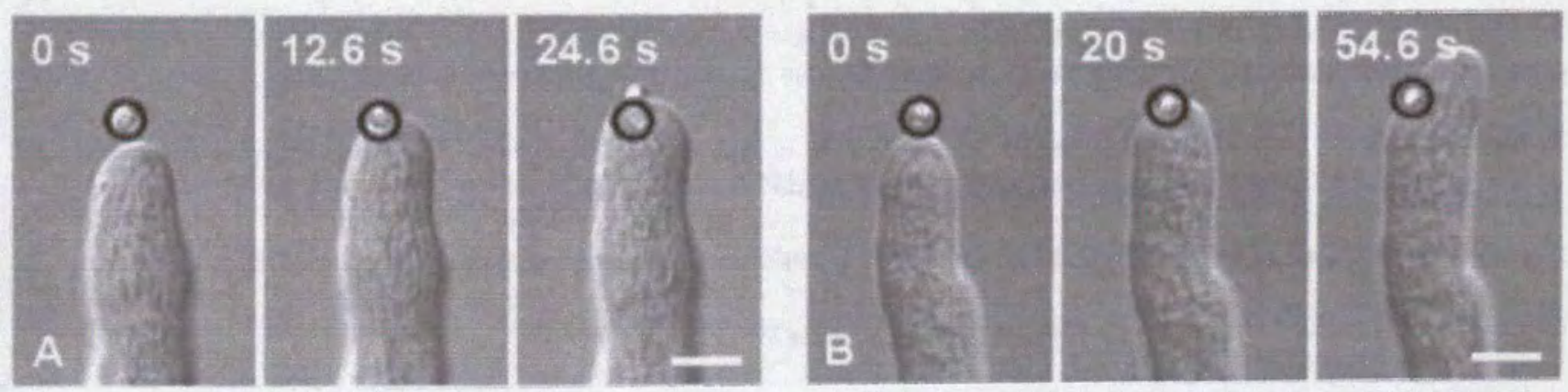

Figure 5: Time courses of growing hyphae showing that $1 \mu \mathrm{m}$ beads trapped with optical tweezers can be pushed out of the trap at a low laser power of $20 \mathrm{~mW}$ (A) but not when the laser power is increased to $70 \mathrm{~mW}$ (B). The circles indicate the position of the optical trap in each frame. Bar $=5 \mu \mathrm{m}$.
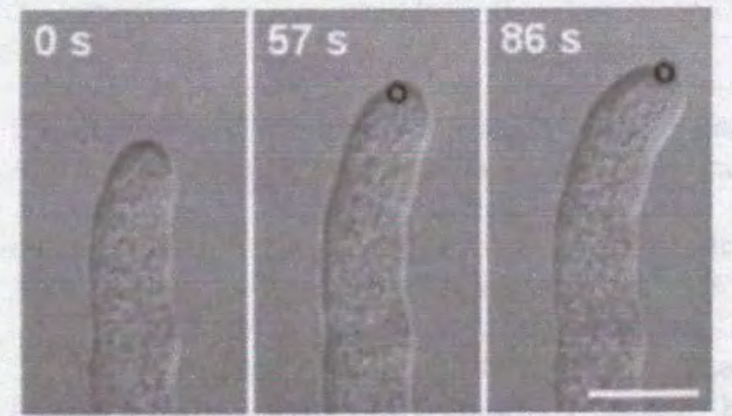

Figure 6: Redirection of growth by the optical trap repelling the Spitzenkörper. The circles indicate the position of the optical trap. Bar $=10 \mu \mathrm{m}$.

\section{DISCUSSION}

The optical tweezers system has proved itself a useful and powerful tool in a number of areas of fungal cell biology ${ }^{12,13,17,18}$ and here we have investigated another potential use for measuring growth forces of fungal hyphae. It was hoped that they will provide an elegant and alternative approach to the miniaturized strain gauges used previously ${ }^{22}$. 
Calibrating the trapping force of the tweezers has shown a linear relationship between laser power and trapping force. Trapping beads and positioning them in the path of a growing hypha has produced some intriguing results. Problems identified in this study, and ways of overcoming these problems in the future are as follows:

(1) Bead size. Using a $1 \mu \mathrm{m}$ bead to measure forces has problems associated with it. The hyphae need only move the bead $0.5 \mu \mathrm{m}$, or less, before the Spitzenkörper will be influenced by the laser. It has been previously reported that the the Spitzenkörper is repelled by the laser, leading to a redirection of hyphal tip growth ${ }^{12.13}$ (Fig. 6). This could cause difficulty in assessing whether a tip has grown round a bead or simply around the laser beam. Larger beads would be subject to larger trapping forces and could therefore be useful. A more powerful laser could also be used in order to increase the trapping forces.

(2) Variation between hyphae. Due to natural biological variation, the growth forces generated by different hyphae may vary. It may be revealing to determine whether different growth forces can be correlated with hyphal width and/or growth rate. Comparisons of the growth forces generated by wide, fast growing leader hyphae at the colony periphery versus those generated by narrow, slow growing germ tube hyphae emerging from spores could be interesting here. The more delicate forces likely to be produced by germ tubes could be measured using optical tweezers.

(3) Trap strength in the $z$ axis. The trapping force of tweezers is weaker in the $z$ (or optic) axis and because the contact is initially between two spherical objects it is to be expected that beads will, at times, take the path of least resistance and 'roll' off the tip in the $z$ direction. Ideally we would like the tip to push against a flat surface but trapping a flat sided object which can be orientated appropriately with the tweezers will probably be difficult to achieve. Using larger beads (e.g. up to $10 \mu \mathrm{m}$ in diameter) would provide a partial solution by presenting flatter surfaces to growing tips.

\section{ACKNOWLEDGEMENTS}

Graham Wright is funded by the EPSRC. We thank Dr. Eirini Theofanidou for providing the schematic in Fig. 2.

\section{REFERENCES}

1. A. Ashkin, J. M. Dziedzic, J. E. Bjorkholm, and S. Chu, "Observation of a single-beam gradient force optical trap for dielectric particles," Opt. Lett., 11, pp. 288-290, 1986.

2. M. P. Sheetz, "Laser Tweezers in Cell Biology," Methods Cell Biol., 55, 1998.

3. S. M. Block, "Making light work with optical tweezers," Nature, 360, pp. 493-495 1992.

4. J. E. Molloy and M. J. Padgett, "Lights, action: optical tweezers," Contemporary Physics, 43, pp. 241-258, 2002.

5. W. H. Wright, G. J. Sonek, Y. Tadir, and M. W. Berns, "Laser trapping in cell biology," J. Quant. Elec,, 26, pp. 2148-2157, 1990.

6. S. C. Kuo and M. P. Sheetz, "Optical tweezers in cell biology," Trends Cell Biol., 2, 116-118, 1992.

7. K. O. Greulich, Micromanipulation by light in biology and medicine: The laser micobeam and optical tweezers, Birkhauser Verlag, Basel, 1999.

8. R. H. Davis, Neurospora: Contributions of a model organism, Oxford Univerity Press, Oxford, 2000.

9. D. D. Perkins and R. H. Davis, "Neurospora at the millennium," Fungal Gent. Biol., 31, pp. 153-167, 2000.

10. J. E. Galagan, S. E. Calvo, K. A. Borkovich, E. U. Selker, N. D. Read, D. Jaffe, W. FitzHugh, L. J. Ma, S. Smirnov, S. Purcell, B. Rehman, T. Elkins, R. Engels, S. Wang, C. B. Nielsen, J. Butler, M. Endrizzi, D. Qui, P. Ianakiev, D. Bell-Pedersen, M. A. Nelson, M. Werner-Washburne, C. P. Selitrennikoff, J. A. Kinsey, E. L. Braun, A. Zelter, U. Schulte, G. O. Kothe, G. Jedd, W. Mewes, C. Staben, E. Marcotte, D. Greenberg, A. Roy, K. Foley, J. Naylor, N. Stange-Thomann, R. Barrett, S. Gnerre, M. Kamal, M. Kamvysselis, E. Mauceli, C. Bielke, S. Rudd, D. Frishman, S. Krystofova, C. Rasmussen, R. L. Metzenberg, D. D. Perkins, S. Kroken, C. Cogoni, G. Macino, D. Catcheside, W. Li, R. J. Pratt, S. A. Osmani, C. P. DeSouza, L. Glass, M. J. Orbach, J. A. Berglund, R. Voelker, O. Yarden, M. Plamann, S. Seiler, J. Dunlap, A. Radford, R. Aramayo, D. O. Natvig, L. A. Alex, G. Mannhaupt, D. J. Ebbole, M. Freitag, I. Paulsen, M. S. Sachs, E. S. Lander, C. Nusbaum, and B. Birren, "The genome sequence of the filamentous fungus Neurospora crassa," Nature, 422, pp. 859-868, 2003. 
11. K. A. Borkovich, L. A. Alex, O. Yarden, M. Freitag, G. E. Turner, N. D. Read, S. Seiler, D. Bell-Pedersen, J. Paietta, N. Plesofsky, M. Plamann, M. Goodrich-Tanrikulu, U. Schulte, G. Mannhaupt, F. E. Nargang, A. Radford, C. Selitrennikoff, J. E. Galagan, J. C. Dunlap, J. J. Loros, D. Catcheside, H. Inoue, R. Aramayo, M. Polymenis, E. U. Selker, M. S. Sachs, G. A. Marzluf, I. Paulsen, R. Davis, D. J. Ebbole, A. Zelter, E. R. Kalkman, R. O'Rourke, F. Bowring, J. Yeadon, C. Ishii, K. Suzuki, W. Sakai, and R. Pratt, "Lessons from the genome sequence of Neurospora crassa: tracing the path from genomic blueprint to multicellular organism," Microbiol. Mol. Biol. Rev., 68, pp. 11082004.

12. G. D. Wright, J. Arlt, W. C. K. Poon, and N. D. Read, "Optical tweezer micromanipulation of filamentous fungi," in prep., 2005.

13. C. E. Bracker, D. J. Murphy, and R. Lopez-Franco, "Laser microbeam manipulation of cell morphogenesis in growing fungal hyphae," Functional Imaging of optical manipulation of living cells, D. L. Farkas, and B. J. Tromberg (ed.), Proc. SPIE, 2983, pp. 67-80, International Society for Optical Engineering, Bellingham, 1997.

14. M. Freitag, P. C. Hickey, N. B. Raju, E. U. Selker, and N. D. Read, "GFP as a tool to analyze the organization, dynamics and function of nuclei and microtubules in Neurospora crassa," Fungal Genet. Biol., 41, pp. 897-910, 2004.

15. P. C. Hickey, D. Jacobson, N. D. Read, and N. L. Louise Glass, "Live-cell imaging of vegetative hyphal fusion in Neurospora crassa," Fungal Genet. Biol., 37, pp. 109-119, 2002.

16. P. C. Hickey, S. R. Swift, M. G. Roca, and N. D. Read, "Live-cell imaging of filamentous fungi using vital fluorescent dyes and confocal microscopy," Methods in Microbiology, 34, pp. 63-87, 2005.

17. M. W. Berns, J. R. Aist, W. H. Wright, and H. Liang, "Optical trapping in animal and fungal cells using a tunable, near-infrared titanium-sapphire laser," Exp. Cell Res., 198, pp. 375-378, 1992.

18. M. G. Roca, J. Arlt, C. E. Jeffree, and N. D. Read, "Cell biology of conidial anastomosis tubes in Neurospora crassa," Eukaryotic Cell, 4, pp. 911-919, 2005.

19. S. D. Harris, N. D. Read, R. W. Roberson, B. Shaw, S. Seiler, M. Plamann, and C. Momany, "Polarisome meets Spitzenkorper: microscopy, genetics, and genomics converge," Eukaryotic Cell, 4, pp. 225-229, 2005.

20. N. P. Money, "The fungal dining habit: a biomechanical perspective," Mycologist, 18, pp. 71-76, 2004.

21. N. P. Money, "Biomechanics of invasive hyphal growth" The Mycota, Volume III, Biology of the Fungal Cell, R. J. Howard and N. A. R. Gow (ed.), Springer-Verlag, New York, 2001.

22. N. P. Money, C. M. Davis, and J. P. Ravishankar, "Biomechanical evidence for convergent evolution of the invasive growth process among fungi and oomycete water molds," Fungal Genet. Biol., 41, pp. 872-876, 2004.

23. R. J. Howard, M. A. Ferrari, D. H. Roach, and N. P. Money, "Penetration of hard substrates by a fungus employing enormous turgor pressures," Proc. Natl. Acad. Sci. USA, 88, pp.11281-11284, 1991.

24. C. Bechinger, K. F. Giebel, M. Schnell, P. Leiderer, H. B. Deising, and M. Bastmeyer, "Optical measurements of invasive forces exerted by appressoria of a plant pathogenic fungus," Science, 285, pp. 1896-1899, 1999.

25. N. Money, "Wishful thinking of turgor revisited: the mechanics of fungal growth," Fungal Genet. Biol., 21, pp. 173$187,1997$.

26. Hickey, P. C. and Read, N. D. "Biology of living fungi," British Mycological Society, Stevenage, 2003

27. K. Svoboda and S. M. Block, "Biological applications of optical forces," Annu. Rev. Biophys. Biomol. Struct. 23, pp. 247-285, 1994. 


\title{
Optical tweezer micromanipulation of filamentous fungi
}

\author{
Graham D. Wright $^{\text {a,b }}$, Jochen Arlt ${ }^{\text {b,c }}$, Wilson C.K. Poon ${ }^{\text {b,d }}$, Nick D. Read ${ }^{a, b, *}$ \\ ${ }^{a}$ Fungal Cell Biology Group, Institute of Cell Biology, University of Edinburgh, Rutherford Building, Edinburgh EH9 3JH, UK \\ ${ }^{\mathrm{b}}$ Collaborative Optical Spectroscopy, Micromanipulation and Imaging Centre (COSMIC), University of Edinburgh, James Clerk Maxwell Building, \\ Edinburgh EH9 $3 \mathrm{JZ}, \mathrm{UK}$ \\ ${ }^{\circ}$ Department of Physics, JJ Thomson Physical Laboratory, The University of Reading, PO Box 220, Whiteknights, Reading RG6 $6 A F, U K$ \\ ${ }^{d}$ SUPA and School of Physics, University of Edinburgh, James Clerk Maxwell Building, Edinburgh EH9 3JZ, UK
}

Received 16 May 2006; accepted 5 July 2006

Available online 5 September 2006

\begin{abstract}
Optical tweezers have been little used in experimental studies on filamentous fungi. We have built a simple, compact, easy-to-use, safe and robust optical tweezer system that can be used with brightfield, phase contrast, differential interference contrast and fluorescence optics on a standard research grade light microscope. We have used this optical tweezer system in a range of cell biology applications to trap and micromanipulate whole fungal cells, organelles within cells, and beads. We have demonstrated how optical tweezers can be used to: unambiguously determine whether hyphae are actively homing towards each other; move the Spitzenkörper and change the pattern of hyphal morphogenesis; make piconewton force measurements; mechanically stimulate hyphal tips; and deliver chemicals to localized regions of hyphae. Significant novel experimental findings from our study were that germ tubes generated significantly smaller growth forces than leading hyphae, and that both hyphal types exhibited growth responses to mechanical stimulation with optically trapped polystyrene beads. Germinated spores that had been optically trapped for 25 min exhibited no deleterious effects with regard to conidial anastomosis tube growth, homing or fusion.
\end{abstract}

(c) 2006 Elsevier Inc. All rights reserved.

Index Descriptors and Abbreviations: Germ tube; Hyphal tip growth; Micromanipulation; Neurospora crassa; Optical tweezers; Organelle; Spitzenkörper

\section{Introduction}

Optical tweezers, also known as optical traps or laser tweezers, were first described in the 1980 s by Ashkin and colleagues (Ashkin et al., 1986). They permit the non-invasive micromanipulation of inert and biological objects by means of optical radiation alone.

Optical tweezers use the forces generated when light interacts with matter to trap objects near the point of focus of an objective lens. To understand the origin of trapping forces for objects interacting with a laser beam, one needs to appreciate that when photons are absorbed, reflected or refracted by an object, the momentum they possess is changed, i.e. the object exerts a force on the photons. New-

\footnotetext{
- Corresponding author. Fax: +441316505392.

E-mail address: Nick@fungalcell.org (N.D. Read).
}

ton's third law states that for every action force there is a corresponding reaction force that is equal in magnitude and opposite in direction. The object exerting the force on the photons will therefore experience a reaction force (Fig. 1A). Optical tweezers require an intense laser light source, tightly focused by an objective lens of high numeri$\mathrm{cal}$ aperture. They produce forces in the piconewton range that are sufficient to trap microscopic particles and move them relative to their surroundings (Fig. 1A and B). For a particle to be trapped efficiently it must have a refractive index that is sufficiently higher than its surroundings. The trapped object can be moved either by moving the microscope stage or by moving the trap position within the field of view (e.g. with galvanometric mirrors). The sizes of particles that can be trapped range from large objects such as whole cells down to particles of a few tens of nanometer in size. The trapping force can be adjusted by changing the laser power. Most recently, multiple traps with positions 

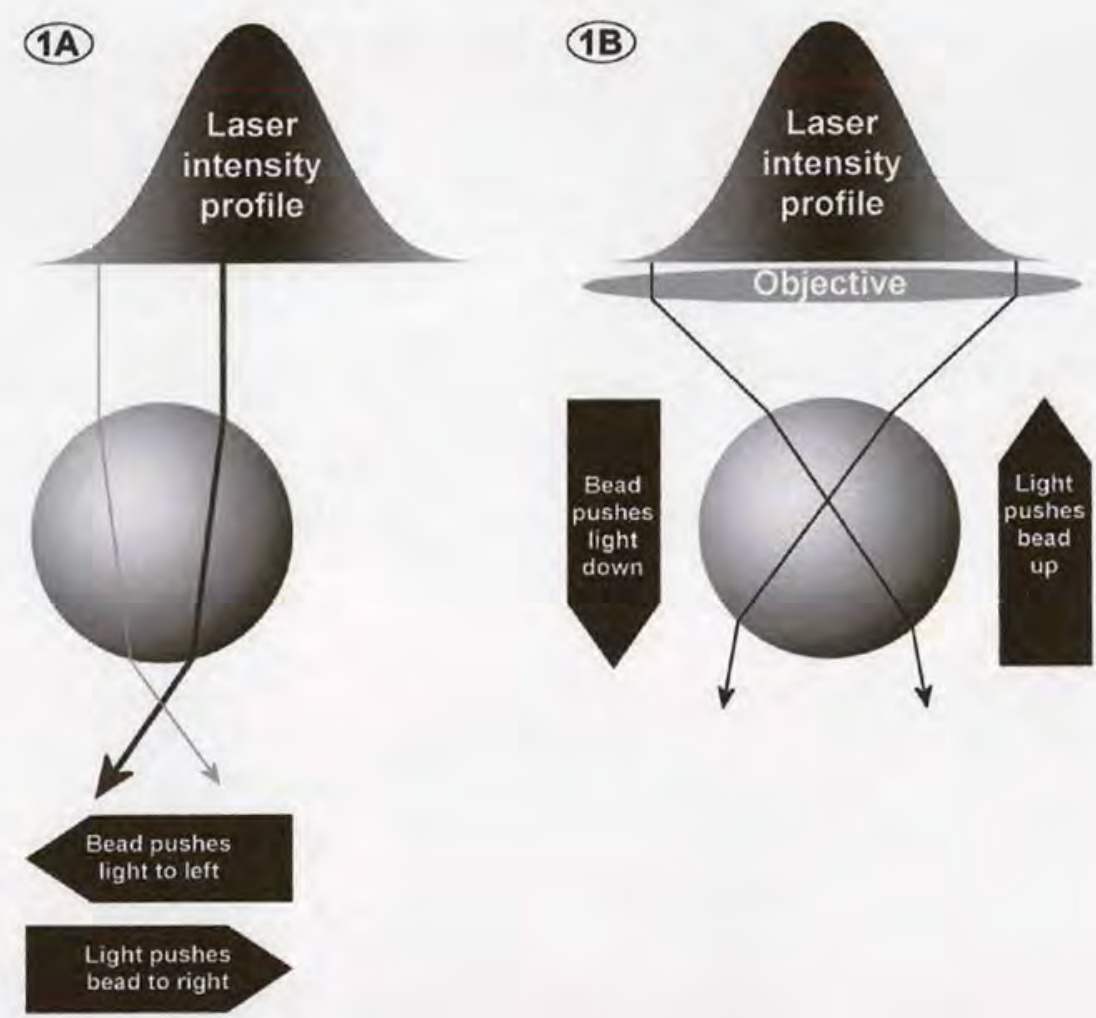

Fig. 1. Ray diagrams showing the interaction of light with a transparent sphere (e.g. a polystyrene bead). The sphere causes a change in direction of the light (an action force) and results in an equal and opposite reaction force on the sphere in accordance with Newton's third law. (A) As the centre of a laser beam with a Gaussian profile is more intense, the ray from this region generates a greater force than the ray from the periphery of the beam. Therefore the net reaction force on the sphere pushes the bead to the right. Upon reaching the centre of the beam the sphere would be trapped in $x$ and $y$ directions but not $z$. (B) To also trap in the $z$ direction the light is focused by an objective lens. In both cases the object is attracted to and trapped in the region of highest light intensity, which is at the point of focus (figure adapted with permission from Dholakia et al., 2002).

controllable in real time have been implemented (Grier and Roichman, 2006). A number of general reviews on optical tweezers are available: Block $(1990,1992)$, Kuo and Sheetz (1992), Ashkin (1997), Sheetz (1998), Greulich (1999), Molloy and Padgett (2002) and Neuman and Block (2006).

Optical tweezer micromanipulation of living cells provides a powerful method for investigating many aspects of cell biology. Optical tweezers can be used to manipulate whole living cells or organelles directly, deliver molecules in microspheres to localized cell regions, or mechanically stimulate cells by pushing trapped objects against them. Using light to manipulate cells has the following advantages over the more traditional mechanical methods of micromanipulation: it is much less invasive and can inflict negligible damage and stress to cells; it allows objects within living cells to be trapped; it offers very precise control of the position of the object being manipulated; and it allows instant trapping or release of objects (Wright et al., 1990; Block, 1990; Berns et al., 1991; Kuo and Sheetz, 1992; Weber and Greulich, 1992; Svoboda and Block, 1994; Sheetz, 1998; Molloy and Padgett, 2002). Optical tweezers have been used in a wide range of biological applications which include: micromanipulating cells to redirect their growth (Leitz et al., 1995; Bracker et al., 1997; Erlicher et al, 2002); micromanipulating organelles within cells
(Berns et al., 1992; Leitz et al., 1995; Tolić-Norrelykke et al., 2005); isolating individual cells, organelles and chromosomes (Leitz et al., 2003; Liu et al., 2004); measuring the forces produced by motor proteins and RNA polymerases (Block et al., 1989; Finer et al., 1994; Greulich, 2005; Kuo and Sheetz, 1993) measuring the biophysical properties of DNA (Cluzel et al., 1996; Wang et al., 1997; Greulich and Pilarczyk, 1998; Baumann et al., 2000; Greulich et al., 2000; Lang et al., 2003); fusing cells (Block, 1992); providing localized mechanostimulation of a cell (Wang et al., 2005); and automating cell sorting (Kuo and Sheetz, 1992).

Whole cells and organelles of budding yeast (Saccharomyces cerevisiae) have been micromanipulated in a range of studies to evaluate different optical tweezer systems (Ashkin, 1991; Grimbergen et al., 1993; Daria et al., 2004; Goksor et al., 2004; Sacconi et al., 2005; Lafong et al., 2006). Optical tweezers have also been used more experimentally with yeast cells to: investigate the viscoelastic properties of the cytoplasm (Tolić-Norrelykke et al., 2004); demonstrate the specification of the division plane by the nucleus in the fission yeast Schizosaccharomyces pombe (Tolić-Norrelykke et al., 2005); and examine the inhibition of growth of Hanseniaspora uvarum cells by confining them with optically trapped cells of Sacch. cerevisiae (Arneborg et al., 2005). 
Filamentous fungi are also well suited to being micromanipulated by optical tweezers, particularly because they can be analyzed as monolayers of germinating spores or growing hyphae, can exhibit rapid growth, can respond quickly to stimuli, and often possess large hyphae or cells. Berns et al. (1992) were the first to use optical tweezers with filamentous fungi, and showed that organelles could be trapped and moved within hyphae without obvious damage. They found that the order of responsiveness of organelles to trapping was: lipid bodies $>$ nucleoli $>$ mitochondria $>$ chromosomes. Bracker et al. (1997) later showed how optical tweezers could be used to move the Spitzenkörper within a hyphal tip, change the direction of hyphal growth, redistribute secretory vesicles in the cytoplasm, and induce branch formation. Their study provided compelling evidence that the Spitzenkörper is responsible for hyphal growth directionality (also see Fig. 3 in Bartnicki-Garcia, 2002). Of particular interest was their observation that rather than being trapped, the Spitzenkörper was repelled by the laser, as was later shown by Wright et al. (2005). The Spitzenkörper is a predominately phase dark structure when observed by phase contrast light microscopy (Lopez-Franco and Bracker, 1996) indicating that, overall, it has a higher refractive index than the surrounding cytoplasm. It had therefore been expected that the Spitzenkörper should be trapped rather than repelled by optical tweezers (Bracker et al., 1997).

Roca et al. (2005) used optical tweezers in a novel assay to assess unambiguously whether conidial anastomosis tubes (CATs) of Neurospora crassa home towards each other or not. This technique allows a conidium or conidial germling to be optically trapped and to have its position relative to another changed. Using this method, Roca et al. (2005) showed that CAT tips were both the sites of chemoattractant secretion and reception. Furthermore, by using mutants defective in signalling, it was shown that cAMP is not the CAT chemoattractant (Roca et al., 2005) and that the mutant soft forms CATs that are unable to home towards or fuse with other CATs (Fleißner et al., 2005).

The first attempts to use optical tweezers to measure the growth forces generated by leading hyphae of $N$. crassa were made by Wright et al. (2005) by placing beads trapped with known forces in their paths. It was concluded that the growth forces of the hyphae analyzed were greater than the piconewton forces which optical tweezers can be used to readily measure. This supported a previous study (Money et al., 2004) in which it was estimated from measurements using a miniaturized strain gauge that vegetative hyphae generate growth forces which are several orders greater than can be measured with optical tweezers.

Our aim in this paper is to evaluate the use of optical tweezers as experimental tools in a range of cell biology applications on filamentous fungi. We designed and built a simple, compact, easy-to-use, safe and robust optical tweezer system that was mounted on a research grade inverted microscope. This system was used to trap and move whole cells, organelles and beads in combination with differential interference contrast (DIC), phase contrast and widefield fluorescence imaging.

\section{Materials and methods}

\subsection{Fungal strains and culture conditions}

The $N$. crassa wild-type strain 74-OR23-1VA (\# 2489, FGSC, Kansas City, KS, USA) was used. It was grown and maintained on solid Vogel's minimal medium (Davis, 2000) in continuous light at $25^{\circ} \mathrm{C}$.

\subsection{Sample preparation}

Macroconidia were harvested from Petri dish cultures of $N$. crassa by pipetting liquid Vogel's medium onto the colony, swirling, and then removing the resulting spore suspension before adjusting its concentration to $1 \times 10^{6}$ macroconidia ml $^{-1}$ using a haemocytometer. A $200 \mu 1$ droplet of this macroconidial suspension was transferred either into an eight well slide culture chamber (Nalge Nunc International, www.nalgenunc.com) or onto coverslips pre-coated with poly (vinyl) alcohol (PVA) which prevents the macroconidia from adhering to the glass (Krylov and Dovichi, 2000).

To image, stain and manipulate hyphal tips, the inverted agar block culture method was used (Hickey et al., 2005). The hyphal vacuolar system was stained with $20 \mu \mathrm{M}$ Oregon Green 488 (DFFDA) (Invitrogen, Molecular Probes, www.probes.invitrogen.com) made up in liquid Vogel's medium. The inverted agar block method was adapted when trapping polystyrene beads which were $4 \mu \mathrm{m}$ in diameter (Interfacial Dynamics Corp., www.idclatex.com). For this purpose the agar block was supported on a coverslip with adhesive electrician's insulation tape. This provided more space for the beads in the liquid layer between the coverslip and agar, and it prevented the beads from adhering to the agar. Beads were also coated with bovine serum albumin (BSA) to prevent them from adhering to the fungal cells. For this purpose, the beads were suspended in a $1 \%$ aqueous solution of BSA (Sigma - www.sigmaaldrich.com) for $10 \mathrm{~min}$ before centrifugation and resuspension in Vogel's liquid medium. $1 \mu \mathrm{m}$ porous silica beads (a kind gift from C.G. Hunt, USDA Forest Products Laboratory, Madison, WI, USA) were used for the localized delivery of a $50 \mu \mathrm{M}$ latrunculin-B (Calbiochem - Merck Biosciences, www.merckbiosciences.co.uk) solution made up in Vogel's liquid medium.

\subsection{Microscopy}

The optical tweezer system was mounted on a Nikon Eclipse TE2000-U inverted microscope. A Nikon plan apo $100 \times, 1.4$ N.A. DIC $\mathrm{H}$ oil immersion objective was used for DIC and fluorescence microscopy, and a Nikon plan fluor $100 \times, 1.3$ N.A. Ph 3 DLL oil immersion objective was used for phase contrast microscopy. To increase the camera's 
field of view, a $0.7 \times$ demagnifying lens was used when necessary. For widefield fluorescence microscopy a mercury vapour lamp with a Nikon B-2A filter cube (containing a $450-490 \mathrm{~nm}$ excitation filter, $500 \mathrm{~nm}$ long pass dichroic mirror, and $515 \mathrm{~nm}$ long pass emission filter) was used.

\subsection{Optical tweezer set-up}

The custom built optical tweezer system (Fig. 2) contains a near-infrared $(\lambda=785 \mathrm{~nm})$ diode laser, the output power of which is adjustable up to $70 \mathrm{~mW}$ (VPSL-0785-070-x-5-A; Blue Sky Research, www.blueskyresearch.com). The laser beam is directed first towards two galvanometric beam steering mirrors and then into the rear of the microscope (Fig. 2A and C). A slight modification made to the microscope, which involved lifting the nosepiece, allows the optical tweezers to be combined with fluorescence imaging. A dichroic mirror reflects the beam into the objective lens. The beam is expanded to fill the back aperture of the objective to offer maximal trapping efficiency. A computer program written in LabVIEW 6.1 (National Instruments, www.ni.com) allows the user to move the trap in $x$ and $y$ directions across the field of view by controlling the galvanometric mirrors at the click of the mouse. The computer takes the images generated by the CCD camera (Applied Vision Technologies, Dolphin 145-F, from FirstSight Vision, www.firstsightvision.co.uk), and moves the trap in a straight line at constant speed to any position in the field of view. The speed of trap movement can be preset in $\mu \mathrm{m} \mathrm{s}^{-1}$ in the tweezer control program. A double click releases the trapped object. Movement in the $z$ axis is made by adjusting the focus of the microscope. It is important to note here that the laser power at the specimen is approximately $50 \%$ of the laser output power due to attenuation through the microscope, particularly the objective lens (Svoboda and Block, 1994; Neuman et al., 1999). In this paper we refer to laser power in terms of output power.

\subsection{Digital image processing and animation}

Images were captured either singly through the control software directly from the camera or as a time course using screen capture software (HyperCam, www.hyperionics. com). Further processing was carried out with Paintshop Pro (v. 7; JASC Software, www.jasc.com). Time courses of
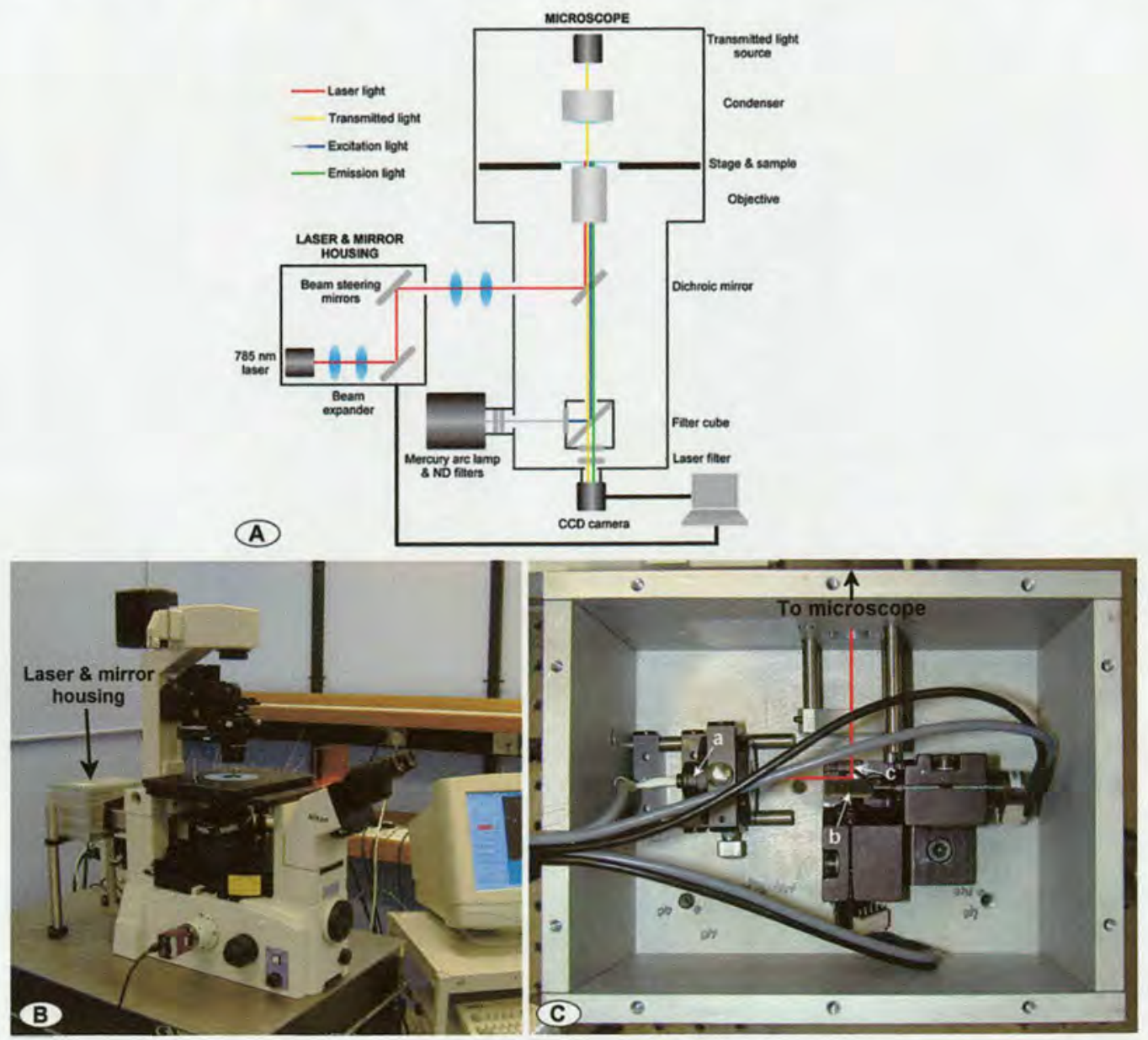

Fig. 2. (A) Schematic diagram of the optical tweezer system used. (B) The tweezer system mounted on the microscope. (C) The interior of the laser and mirror housing in which (a) is the $785 \mathrm{~nm}$ diode laser, (b) are the galvanometric mirrors, and (c) is the path taken by the laser beam. The optical elements required for phase contrast, DIC and fluorescence can be incorporated into the microscope whilst maintaining the full functionality of the optical tweezers. 
images were edited and built up into animation movies (.avi and mpeg files) using Animation Shop (v. 3; JASC Software, www.jasc.com), Premier Pro (v. 1.5; Adobe, www.adobe.com), and ImageJ (freeware; http:/l rsb.info.nih.gov/ij/).

\section{Results}

\subsection{Manipulation of whole cells}

Ungerminated macroconidia (Fig. 3) and germinated macroconidia (Fig. 4) were trapped and moved around relative to their surroundings. The semi-spherical, ungerminated macroconidia were easily moved within the field of view in $x$ and $y$ directions with the computer-controlled steerable trap controlled by the galvanometric mirrors (Supplementary movie 1 at www.fungalcell.org/tweezermovies.htm). Macroconidia could be moved over greater distances by trapping them in a fixed position and then carefully moving the microscope stage. Using a laser output power of $40 \mathrm{~mW}$ it was possible to move a macroconidium at a speed of up to $15 \mu \mathrm{m} \mathrm{s}^{-1}$ in $x$ and $y$ directions, without risking losing the macroconidium from the trap. The speed of movement achievable varied depending on the size and optical properties of a given macroconidium, the laser power used, and the viscosity of the medium. With time, macroconidia tended to adhere to the coverslip, after which they could not be detached from the glass surface even when using the highest output laser power $(70 \mathrm{~mW})$. To overcome this problem the coverslips were coated with PVA, which prevented spore adhesion to the glass without perturbing germ tube growth. Germ tubes up to $100 \mu \mathrm{m}$ long could be trapped at different positions along their lengths, thereby allowing control of their orientation (Fig. 4; Supplementary movie 2 at www.fungalcell.org/ tweezermovies.htm).

Recently a new type of hypha, the conidial anastomosis tube (CAT), has been described (Roca et al., 2005). CATs arise from conidia or germ tubes and home towards and fuse with each other. Optical tweezers have been used to trap and move one CAT relative to another to unambiguously determine whether the two CATs are homing towards each other or not. This technique is being used to

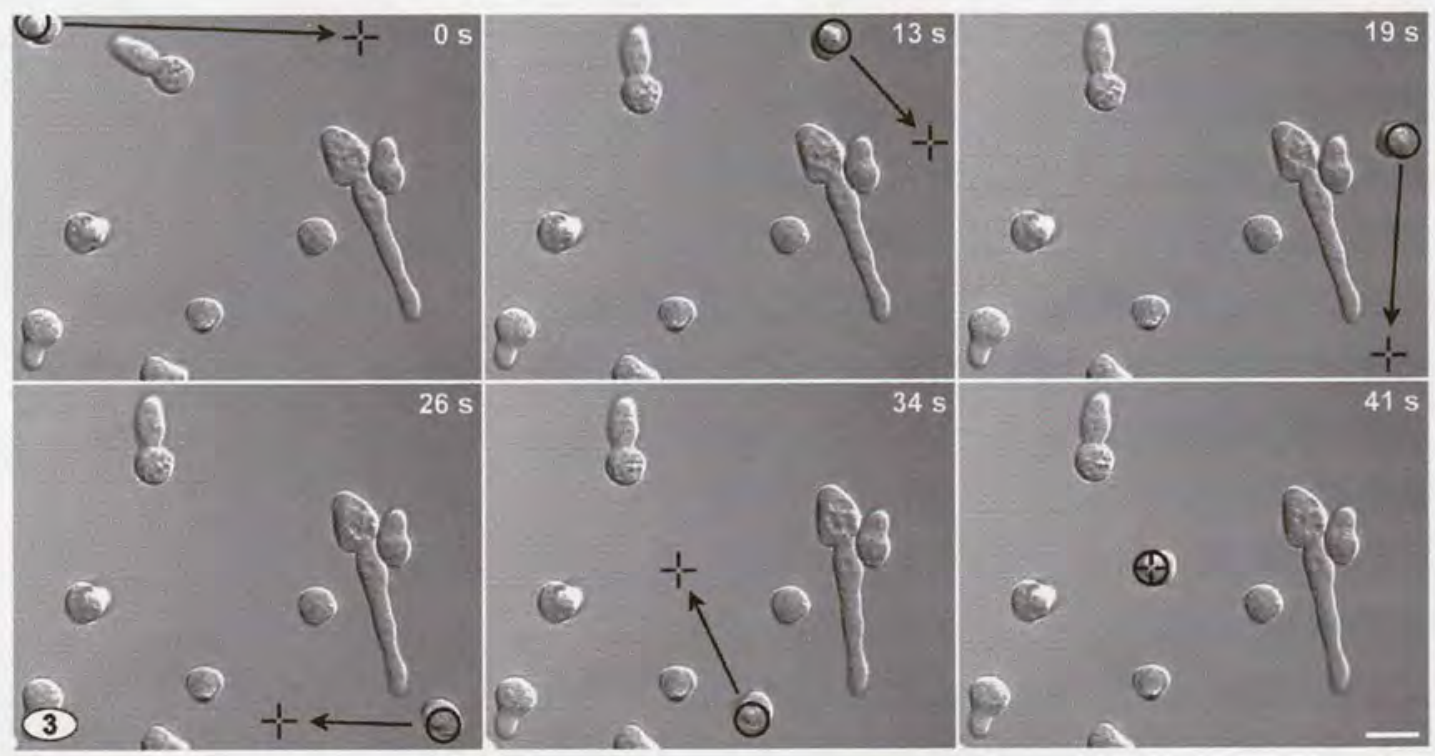

Fig. 3. Micromanipulation of an ungerminated macroconidium of Neurospora crassa. The circle represents the current position of the laser whilst the cross-hair shows the position to which the trap is about to be moved. Bar $=10 \mu \mathrm{m}$. (See Supplementary movie 1 at www.fungalcell.org/tweezermovies.htm.)

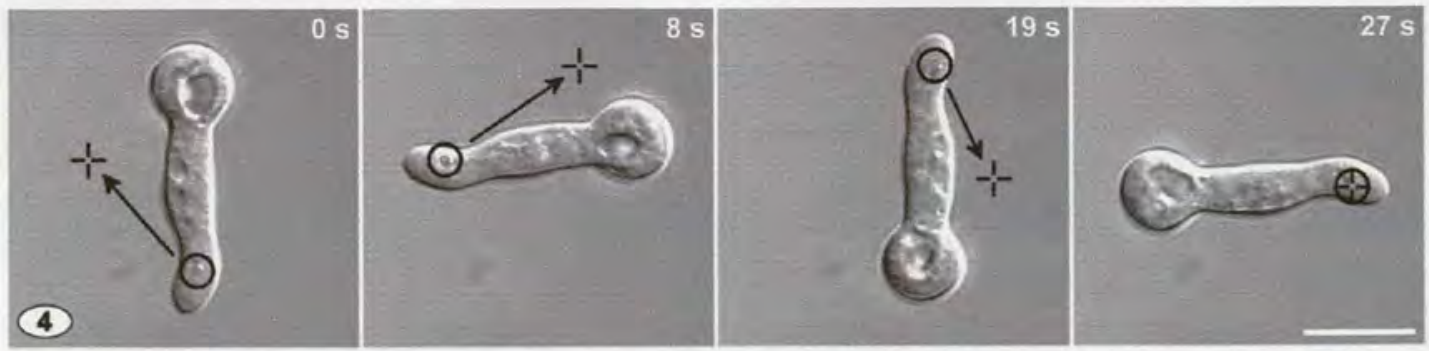

Fig: 4. Rotation of a macroconidial germling of $N$. crassa. The circle represents the current position of the laser whilst the cross-hair shows the position to which the trap is about to be moved. Note that as the germling was manipulated it rotated around its centre point and the field of view remained constant throughout the time course. Bar $=10 \mu \mathrm{m}$. (See Supplementary movie 2 at www.fungalcell.org/tweezermovies.htm.) 
study the signalling pathways involved in CAT homing, particularly by using mutants (Fleißner et al., 2005; Roca et al., 2005). In order to conduct this assay, conidia or germlings were previously only trapped for up to $1 \mathrm{~min}$. Here we found that we could extend the trapping time to $25 \mathrm{~min}$ and found that CATs continued to home towards and fuse with each other, without any apparent ill effects (Fig. 5).

\subsection{Manipulation of organelles}

Organelles of high refractive index (e.g. Woronin bodies) visualized by differential interference contrast (DIC) or phase contrast optics were readily trapped within living cells (Fig. 6; Supplementary movie 3 at www.fungalcell.org/ tweezermovies.htm). For some organelles it was necessary to hold the organelle in the trap for some time, during which period it seemed that the organelle ceased to be attached to the cytoskeleton, before it became possible to move the organelle around within the cytoplasm. Some Woronin bodies were found to be strongly tethered to the lateral plasma membrane adjacent to septa such that the tweezer trapping forces were insufficient to move them. In order to move organelles within the viscous hyphal cytoplasm it was necessary to move the trap slowly $(\sim 1-$ $2 \mu \mathrm{m} \mathrm{s}^{-1}$ ) so as not to lose the organelle from the trap. Upon the release of an organelle from the trap it was common to observe the organelle 'float' around in the cytoplasm or move with the bulk cytoplasmic flow (Lew, 2005). Some unidentified, refractile organelles, similar to those described as 'vesicles' by Riquelme et al. (2002), were observed mov- ing tens of micrometers in straight lines within hyphae. These organelles were probably tethered by motor proteins to microtubules. It was often possible to optically trap these organelles but it was apparent that their movement was force-driven. As a result, they were sometimes lost from the trap, particularly when using the trapping laser with low power (data not shown). Moving trapped organelles in a direction that was more-or-less at right angles to the direction in which they were moving along putative microtubules/microfilaments commonly resulted in the organelles being lost from the trap and 'springing back' to the putative cytoskeletal element.

Organelles of lower refractive index than their surroundings were repelled by the trap. Fig. 7A-C shows vacuoles, which are of lower refractive index than the cytoplasm, being moved around within hyphae whilst being observed with phase contrast, DIC or fluorescence microscopy. To move the vacuoles, a technique of 'chasing' was employed, much akin to repelling magnets, in which the vacuoles were pushed away from the trap when it got close to them (Fig. 7A; Supplementary movie 4 at www.fungalcell.org/ tweezermovies.htm). Applying the trap directly to a vacuole resulted in it 'popping' out of the trap (Fig. 7B; Supplementary movie 5 at www.fungalcell.org/tweezermovies.htm). If vacuoles were pressed against the plasma membrane, it was possible to split them in two (Fig. 7C; Supplementary movie 6 at www.fungalcell.org/tweezermovies.htm).

Using what appeared superficially to be the same 'organelle chasing' technique, it was possible to influence the position of the Spitzenkörper within the growing tip, as

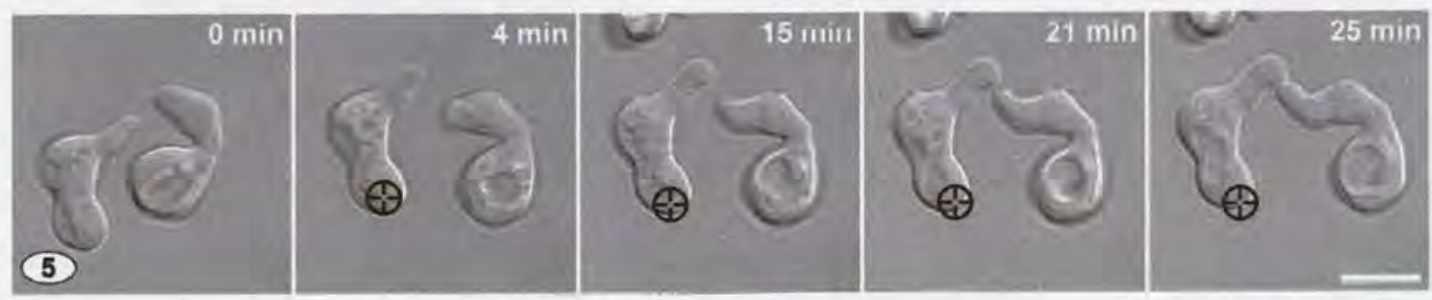

Fig. 5. An example of a conidial anastomosis tube (CAT) homing assay. The two conidia had germinated and their CATs were homing towards each other $(0 \mathrm{~min})$. The left hand germling was repositioned (here shown $4 \mathrm{~min}$ after repositioning). The CAT tips then changed their orientation to home back towards each other $(15$ and $21 \mathrm{~min}$ ) before making contact $(25 \mathrm{~min})$ and subsequently fusing (not shown). The left hand conidium remained trapped throughout the entire $25 \mathrm{~min}$ period without apparent inhibition of CAT growth, homing or fusion. The position of the trap is represented by the crosshair in the circle. $B a r=10 \mu \mathrm{m}$.
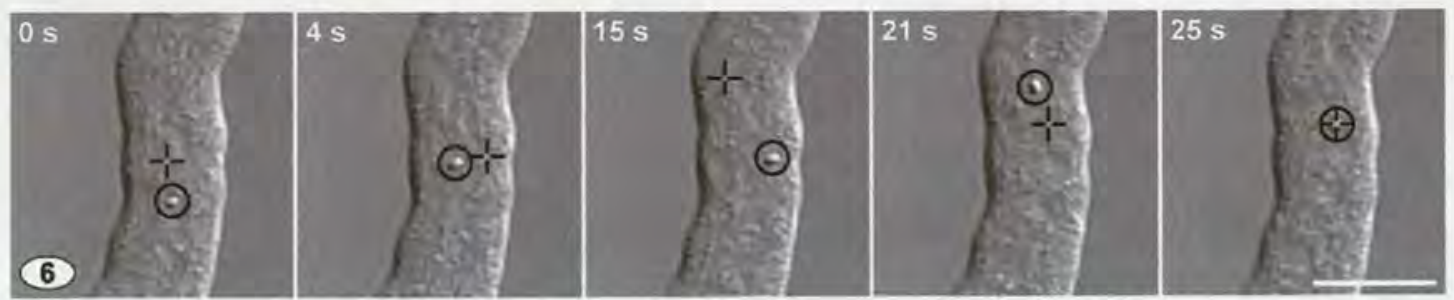

Fig. 6. Trapping and moving a Woronin body within a hypha of Neurospora crassa. The hyphal region shown was just behind the hyphal tip, and is where Woronin bodies can be found floating free in the cytoplasm and not, as is more usual, associated with the hyphal cell cortex or septum (Markham and Collinge, 1987; Jedd and Chua, 2000). The Woronin body could be moved in the direction of the prevailing bulk cytoplasmic flow (towards the hyphal tip), laterally across the hypha and against the flow. The circle represents the current position of the laser whilst the cross-hair shows the position to which the trap is being moved to. Bar $=10 \mu \mathrm{m}$. (See Supplementary movie 3 at www.fungalcell.org/tweezermovies.htm.) 

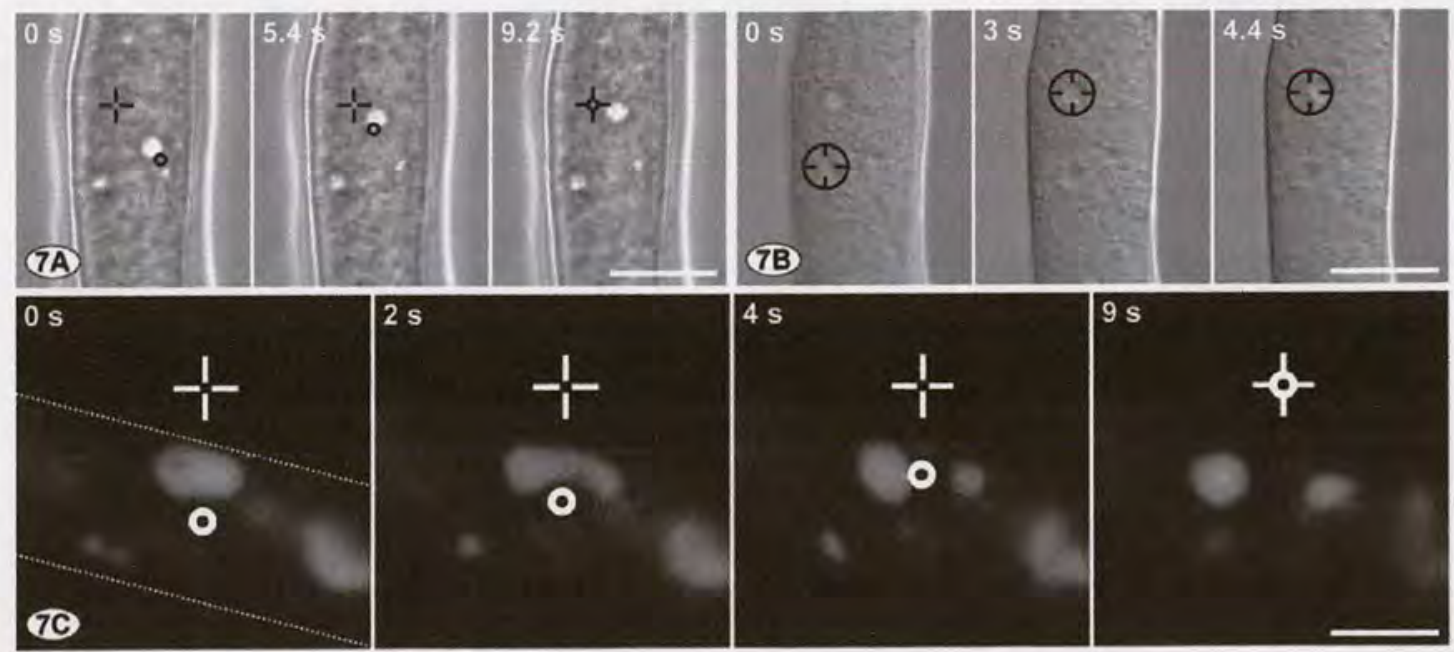

Fig. 7. Repulsion of vacuoles which have a lower refractive index than their surrounding cytoplasm in Neurospora crassa. (A) Vacuoles are visualized as bright objects (phase light) with phase contrast optics, (B) as depressed 'hollows' with DIC optics, or (C), as brightly fluorescent objects when stained with Oregon Green 488 (DFFDA) and imaged with widefield fluorescence microscopy. If the laser was applied directly to the vacuole they 'popped out' of the trap (B, compare the 3 and $4.4 \mathrm{~s}$ time points). As a result of being repelled, the vacuole could be pushed around the cytoplasm (A). If the vacuoles were pressed against the plasma membrane, it was possible to split them into two (C). The outline of the hypha in (C) is indicated by dotted lines. Bar $=10 \mu \mathrm{m}$ (A and $\mathrm{B}$ ). Bar $=5 \mu \mathrm{m}(\mathrm{C})$. (See Supplementary movie 4-6 at www.fungalcell.org/tweezermovies.htm.)

previously described (Bracker et al., 1997; Wright et al., 2005). Placing the trap to the side of the Spitzenkörper resulted in the redirection of growth on the side of the hyphal tip away from the trap (Fig. 8; Supplementary movie 7 at www.fungalcell.org/tweezermovies.htm).

\subsection{Manipulation of microspheres}

Polystyrene beads and other types of transparent microspheres can usually be efficiently trapped, and can provide powerful experimental tools in live-cell studies. Here we have explored their uses in making growth force measurements, generating mechanical stimuli, and providing localized extracellular sources of molecules.

A bead situated at the centre of a laser trap experiences no force. As it is displaced from the trap centre, a restoring force develops that is initially proportional to the displacement, i.e. the trap acts as an ideal ('Hooke's law') spring. Eventually, however, the trap 'softens' and the force decreases to zero: the bead escapes the trap beyond a maxi- mum displacement. Precise measurement of the position of the trapped bead allows the determination of the force exerted on the bead (Sheetz, 1998). On the other hand, an estimate of the forces involved can conveniently be obtained by measuring the maximum trapping force, which increases linearly with the incident laser power (Wright et al., 2005). This is achieved by trapping a bead at a given laser power and then dragging it through a liquid of known viscosity at increasing velocity until it escapes from the trap (Supplementary movie 11 at www.fungalcell.org/tweezermovies.htm). Applying Stoke's law to calculate the drag at the 'escape speed' allows us to calculate the maximum trapping force at that laser power. As shown in Fig. 9, and as has been previously reported by Wright et al. (2005), the growth force of a leading hypha of $N$. crassa is sufficient to push a $4 \mu \mathrm{m}$ polystyrene bead out of a trap even when using the highest laser power (Fig. 9; Supplementary movie 8 at www.fungalcell.org/tweezermovies.htm). Performing the same experiment using a germ tube yielded very different results (Fig. 10). The tip of a germ tube, which grows much
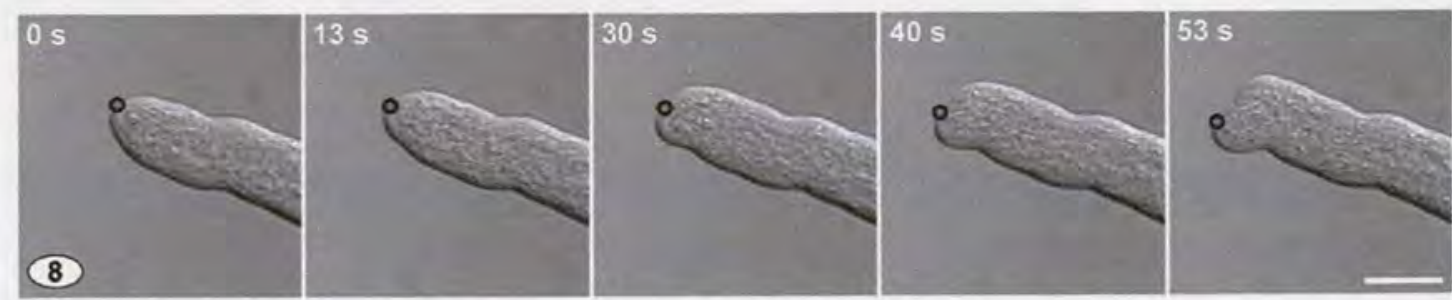

Fig. 8. Redirection of growth by manipulating the Spitzenkörper. The Spitzenkörper is repelled by the laser. When the laser was located to the side of the Spitzenkörper, this resulted in the redirection of hyphal tip growth away from the trap. In this experiment, the trap was repositioned gradually to maintain its position just to the side of the Spitzenkörper as growth progressed. The position of the trap is represented by the circle. Bar $=10 \mu \mathrm{m}$. (See Supplementary movie 7 at www.fungalcell.org/tweezermovies.htm.) 


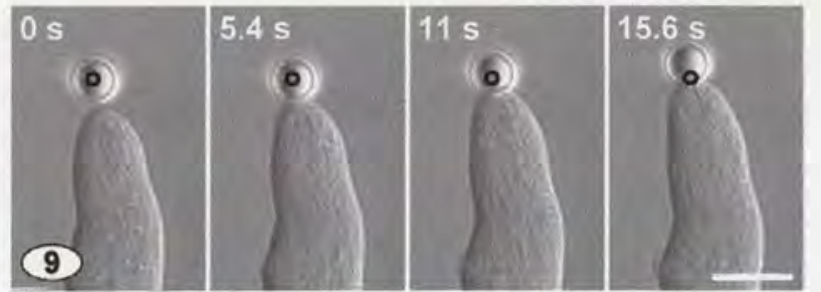

Fig. 9. Inability to halt the growth of a leading hypha with an optically trapped bead. Using an optically trapped $4 \mu \mathrm{m}$ polystyrene bead as an obstacle, the tip of this leading hyphae was able to push the bead out of the trap. The highest output laser power $(70 \mathrm{~mW})$ was used in this experiment, which equates to a trapping force of $19 \mathrm{pN}$ (Wright et al., 2005). The circle represents the position of the laser. Bar $=10 \mu \mathrm{m}$. (See Supplementary movie 8 at www.fungalcell.org/tweezermovies.htm.)

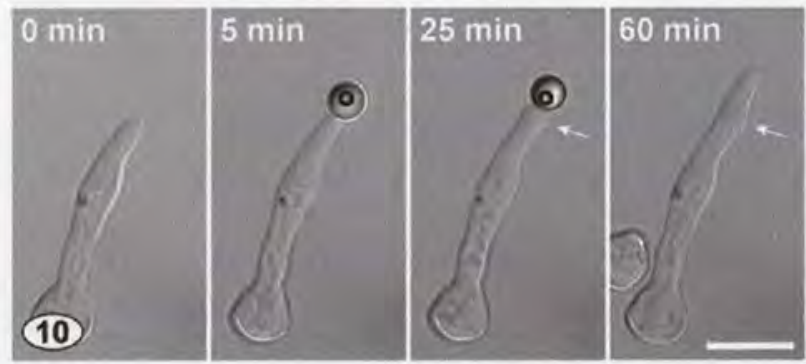

Fig. 10. Inhibition of germ growth with an optically trapped $4 \mu \mathrm{m}$ bead used as an obstacle in front of a germ tube. Note that upon first making contact with the bead the germ tube tip begins to push the bead slightly forward (at $5 \mathrm{~min}$ ), but is unable to push it out of the trap. The tip then proceeds to swell (arrow at $25 \mathrm{~min}$ ). When the obstacle was removed growth resumed at the germ tube tip, leaving a subapical swelling (arrow at $60 \mathrm{~min})$. The highest output laser power $(70 \mathrm{~mW})$ was used in this experiment, which equates to a trapping force of $19 \mathrm{pN}$ (Wright et al, 2005). $B a r=10 \mu \mathrm{m}$. (See Supplementary movie 9 at www.fungalcell.org/ tweezermovies.htm.)

slower than leading hyphae, became swollen upon contact with a bead trapped at full laser power, and then stopped growing. This suggests that germ tubes produce a smaller growth force than leading hyphae. Upon removal of the obstructing bead, growth resumed at the germ tube tip, leaving a subapical swelling (Fig. 10; Supplementary movie 9 at www.fungalcell.org/tweezermovies.htm).

Another method of applying a mechanostimulus to growing hyphal tips was attempted. This involved using a $10 \mu \mathrm{m}$ bead to repeatedly hit a growing tip of a leading hypha by moving the trapped bead at high speeds (up to $40 \mu \mathrm{m} \mathrm{s}^{-1}$ ) back and forth against the hyphal tip. The hyphae treated in this way continued to maintain a uniform linear rate of extension but some slight redirection of growth was sometimes observed following the time point at which the stimulus was applied (Fig. 11; Supplementary movie 10 at www.fungalcell.org/tweezermovies.htm).

Optical tweezers were used to deliver chemicals to localized cellular regions. In Fig. 12A latrunculin-B, a drug inhibiting actin polymerization (Spector et al., 1983), was delivered selectively in a group of three porous silica beads to a hyphal tip. The extension rate of this hypha was dra-

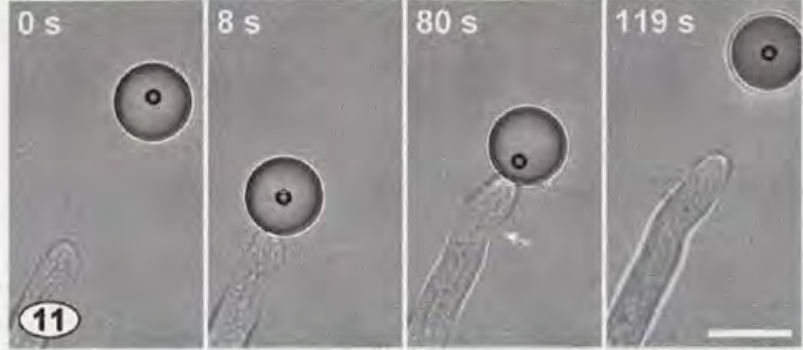

Fig. 11. Effect of repeatedly hitting a tip of a growing vegetative hypha with a $10 \mu \mathrm{m}$ polystyrene bead. Note that the hypha continued to grow (with an extension rate of $7.7 \mu \mathrm{m} \mathrm{min}^{-1}$ ) throughout the period of mechanostimulation but there was a slight redirection of growth during the time (arrow at $80 \mathrm{~s}$ ) that the hypha was stimulated. Bar $=10 \mu \mathrm{m}$. (See Supplementary movie 10 at www.fungalcell.org/tweezermovies.htm.)
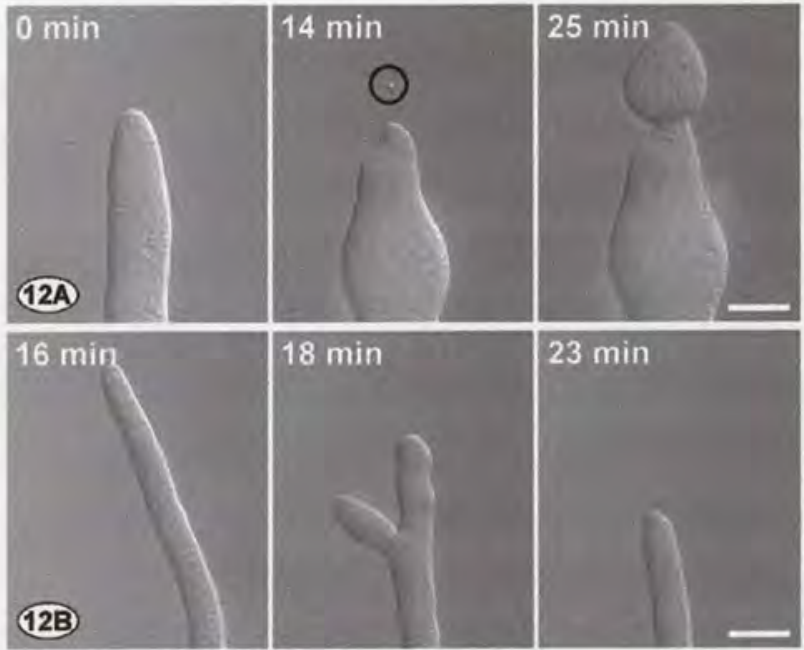

Fig. 12. Using trapped beads to deliver chemicals to a localized region of a cell. (A) Delivery of a localized dose of latrunculin-B to a hyphal tip from a group of three porous silica beads. The drug, which disrupts actin polymerization, has caused hyphal tip growth to be significantly inhibited and to be accompanied by a gross swelling of the hyphal tip region. (B) A neighbouring hypha, which was $\sim 300 \mu \mathrm{m}$ away from the treated hyphae, was unaffected during the period of this experiment, and is shown branching at time $18 \mathrm{~min}$. In both (A) and (B) the field of view has been moved in order to keep the growing hyphal tips in the field of view. $B a r=10 \mu \mathrm{m}$.

matically reduced and as it continued to grow slowly it underwent significant swelling. The growth of neighbouring hyphae up to $\sim 300 \mu \mathrm{m}$ away continued to grow normally during the $30 \mathrm{~min}$ period of this experiment (Fig. 12B). The porous beads, having been soaked in latrunculin-B, were added to the edge of the agar block then trapped and taken to the hyphal tip through the liquid growth medium between the agar block and the coverslip.

\subsection{Does the laser adversely affect trapped cells?}

A spore germination assay to assess possible damage caused to cells by the trapping laser was performed. This involved analyzing the germination of a population of macroconidia in which 50 spores were individually exposed to 


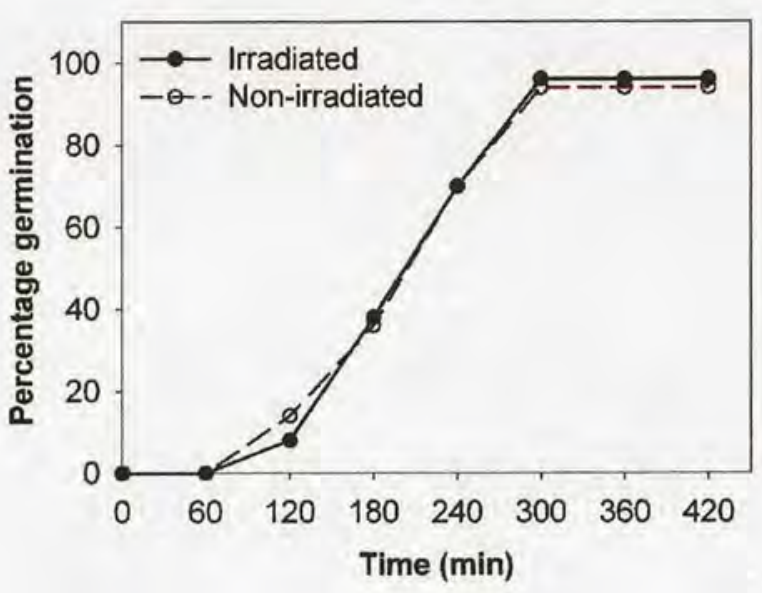

Fig. 13. Macroconidial germination was not significantly affected in spores optically trapped compared with those which were not. Fifty freshly inoculated ungerminated spores were each trapped for $30 \mathrm{~s}$ and their germination followed for $7 \mathrm{~h}$. Similar results were obtained when this experiment was repeated three times.

the trapping laser for $30 \mathrm{~s}$. Their rate of germination was compared to that in a non-irradiated population of macroconidia as a control (Fig. 13). No significant difference in the timing of the onset of germination, the rate of germination or final percentage of germinated spores was found.

\section{Discussion}

This study has demonstrated that optical tweezers are a powerful tool for fungal cell biology research. We have shown that optical tweezers give us the ability: to manipulate whole cells, altering their position relative to one another to study the interaction between them; to manipulate organelles within cells; and apply localized forces, mechanical stimuli or doses of chemicals to cells. All this can be achieved without significant damage to the cells being micromanipulated. The potential opportunities for novel experimentation with optical tweezers when combined with live-cell imaging are vast.

Both ungerminated macroconidia and macroconidial germlings with germ tubes up to $100 \mu \mathrm{m}$ long have been trapped and moved in a very controlled manner. In these cases, a region of the germ tube of high refractive index is trapped sufficiently strongly to allow the whole germ tube to be moved. Moving one CAT relative to another using optical tweezers has formed the basis of a novel CAT homing assay developed to investigate cell signalling between CATs (Fleißner et al., 2005; Roca et al., 2005). In our previous studies, conidial germlings were only trapped for up to $1 \mathrm{~min}$ because of concern that longer periods of irradiation might be deleterious to the cells. However, a big problem we have encountered is that untrapped CATs tend to drift and rotate and their relative positions to each other can change independently of tweezer micromanipulation (Fleißner et al., 2005). Here, we have shown here that it is possible to continuously trap conidia for periods of at least
$25 \mathrm{~min}$ without any discernible effects on CAT growth or homing. Long term trapping prevents the problem of the drifting of individual CATs. Furthermore, we have recently developed a two-trap optical tweezer system which allows us to prevent the drifting and rotation of both CATs in a homing assay (unpublished results).

The $785 \mathrm{~nm}$ laser used in this study did not cause any discernible damage to fungal cells even when used at its full output power $(70 \mathrm{~mW})$. One previous study highlighted two wavelengths ranges, $800-850 \mathrm{~nm}$ and $950-990 \mathrm{~nm}$, which were the least harmful to the cloning efficiency of Chinese Hamster Ovary (CHO) cells. The same study reported that $740-760 \mathrm{~nm}$ and $\sim 900 \mathrm{~nm}$ were the most harmful. The duration of exposure, irrespective of wavelength, was also shown to be a significant factor in a cell's ability to survive (Liang et al., 1996). The results from two further studies were consistent with these findings. Photodamage to bacterial cells of Escherichia coli was found to be minimal at $830 \mathrm{~nm}$ and $970 \mathrm{~nm}$ and maximal at $870 \mathrm{~nm}$ and $930 \mathrm{~nm}$ (Neuman et al., 1999). Cells of the fission yeast Schizosacch. pombe were found to exhibit visible photodamage at $880 \mathrm{~nm}$ but not $830 \mathrm{~nm}$. Despite this, trapping lipid granules in these yeast cells with $830 \mathrm{~nm}$ light at high laser power (56 times that used in our study) delayed cell division (Sacconi et al., 2005). In the latter study, a temperature-sensitive process, mitotic spindle elongation, was used to demonstrate that the yeast cells were not substantially heated by the $830 \mathrm{~nm}$ trapping laser. Photodamage can potentially be caused by the photochemical generation of reactive oxygen species, two-photon absorption (even from a continuous wave laser of the type used in this study), or transient local heating (Neuman et al., 1999; Sacconi et al., 2005). Reducing photodamage to a minimum requires one to judiciously select an optimal combination of laser wavelength, laser power, and the duration of exposure to the laser beam, as well as taking into account the type of biological sample being irradiated (Liang et al., 1996).

Fungal organelles of higher refractive index than their surrounding cytoplasm (e.g. Woronin bodies) are usually trapped whilst organelles of lower refractive index (e.g. vacuoles) are repelled by the trapping laser. Essentially organelles that are visible when viewed microscopically, due to their refractive index being different from that of the surrounding cytoplasm, can be manipulated by optical tweezers unless they are tethered to other cell components. Trapped organelles with high refractive indices can be moved through the naturally viscous cytoplasm at slow speeds. Organelles with lower refractive indices are repelled by the trap and can be pushed or chased around in the cytoplasm. Organelles or particles that are below the resolution of what can be discerned with a light microscope can also be optically trapped if they can exert a force on the photons of the laser beam by absorbing, reflecting or refracting these photons. In this respect, Bracker et al. (1997) used optical tweezers to move locally high concentrations of secretory vesicles, which individually were not resolvable with the light microscope, to new locations 
within hyphal tips and, as a result, induced the formation of new branches at those sites. Use of the repulsion method to move organelles of low refractive index is more difficult and less precise to control but still can be a useful technique for organelle micromanipulation. This method also enabled larger spherical vacuoles to be split in two when they were pushed against the plasma membrane.

One apparent exception to the ability of high refractive index organelles being trapped related to the micromanipulation of the Spitzenkörper. As previously reported, the Spitzenkörper in many fungal species is predominantly of high refractive index and is visually phase dark (Girbardt, 1957; Lopez-Franco and Bracker, 1996; Harris et al., 2005). However, it has been previously reported that the Spitzenkörper is repelled by the trapping laser (Bracker et al., 1997; Wright et al., 2005). Closer analysis of the Spitzenkörper of $N$. crassa reveals it to possess a more complex composite structure containing regions of both high and low refractive index (Lopez-Franco and Bracker, 1996; Harris et al., 2005). This may go someway to explaining why the Spitzenkörper is repelled. Another possible cause of the negative phototropic response of hyphal tips may be because it is photoreceptor-mediated, and involves a photoreceptor in the plasma membrane that is linked via a signal transduction pathway to the Spitzenkörper. Two red/far red light phytochrome-like photoreceptor proteins have been identified as being encoded in the $N$. crassa genome (Galagan et al., 2003; Froehlich et al., 2005).

We found that it was not always possible to move organelles within hyphae with optical tweezers. Some organelles were clearly tethered to other cell components (e.g. cytoskeletal elements) or the plasma membrane. Indeed, cellular organelles are commonly transported along microtubules and actin microfilaments by means of motor proteins (Steinberg, 2000; Lee and Plamann, 2001; Xiang and Plamann, 2003). The tethering of Woronin bodies was demonstrated in Nectria haematococca by Berns et al. (1992). They described the trapping and moving of Woronin bodies up to a distance of $2 \mu \mathrm{m}$ from a septum before the Woronin bodies were lost from the trap and sprung back to the septum. They inferred that Woronin bodies were tethered close to septa by an unidentified elastic filament which was not visible in the light microscope. The Woronin bodies manipulated in the present study were located close to the hyphal tip in the apical hyphal compartment and were not associated with septa or cell cortex. These Woronin bodies were mostly untethered and could be readily moved within the hyphal cytoplasm.

Optical tweezers are frequently used to manipulate transparent beads or microspheres of different sizes, chemical composition or physical properties (Block, 1990; Greulich, 1999; Sheetz, 1998) In this study we used them to: make preliminary measurements of the 'growth forces' generated by the growing tips of germ tubes; mechanically stimulate hyphal tips; and deliver chemicals to localized regions of hyphae.
We previously found that the trapping forces which can be generated by our laser tweezer system with $4 \mu \mathrm{m}$ beads are in the range of $1-19 \mathrm{pN}$. We showed here and previously (Wright et al, 2005), that the growth force of a leading hypha of $N$. crassa is sufficient to push a $4 \mu \mathrm{m}$ polystyrene bead out of a trap even when using the highest laser power (equivalent to a trapping force of $19 \mathrm{pN}$ ). However, the extension of germ tubes was inhibited by a bead of similar size trapped with a similar force. This indicates that the growth force generated by a germ tube is less than that of a leading hypha. This is consistent with results obtained by Money et al. (2004) who estimated from measurements using a miniaturized strain gauge that vegetative hyphae generate growth forces which are several orders greater than those that can be measured with optical tweezers. The reason why we used $4 \mu \mathrm{m}$ beads rather than smaller ones (e.g. $1 \mu \mathrm{m}$ beads) was to avoid the problem of directly irradiating and potentially influencing the behaviour of the Spitzenkörper with the laser trap. In future studies it will be necessary to determine the force per unit area experienced by the germ tube tip. This will require high resolution imaging of the contact area (e.g. by using low-temperature scanning electron microscopy, Read, 1991).

When germ tubes made contact with a trapped bead they exhibited a swelling growth response. Fungal hyphae have been previously found to show a range of responses to physical mechanostimuli (Read et al., 1992). These include asymmetrical cell growth or spore germination in response to contact with a surface (Kwon et al., 1991; Read et al., 1992; Kuo and Hoch, 1996), directional growth and infection structure differentiation in response to microtopographical signals (Hoch et al., 1987; Read et al., 1992; Gow, 1994; Gow et al., 1994; Read et al., 1997), multicellular infection plaque differentiation in response to compression (Lucas, 2004), and intracellular calcium changes in response to shaking (Nelson et al., 2004; Bencina et al., 2005). How germ tubes sense and respond to trapped beads is not known. However, we have recently found that mechanical perturbation (by shaking in liquid culture) of germ tubes often causes them to exhibit a swelling response and this is preceded by a transient increase in cytosolic free calcium (Marris, P.I., Hickey, P.C., Read, N.D., unpublished results). This suggests that the mechanostimulus may be transduced intracellularly by calcium signalling.

The localized delivery of controlled doses of specific chemicals to different cellular regions is another way to use optically trapped beads. In this study we demonstrated how this could be performed by using porous silica beads soaked in the actin polymerization inhibitor, latrunculin-B (Spector et al., 1983). As a result of this treatment, the extension rate of hyphae was inhibited and they underwent significant swelling in contrast to hyphae that were $\sim 300 \mu \mathrm{m}$ distant that continued to grow normally. In the future, this could be a very powerful method to deliver chemicals to localized regions of fungal cells when used in combination with live-cell imaging. Porous beads soaked in pharmacological inhibitors and agonists, caged compounds 
or fluorescent dyes could be used for this purpose. In our study this technique was used rather crudely and needs some improvement and refinement. It would he highly desirable, for instance, for the beads/microspheres/nanocapsules to retain the chemical until it needs to be released, and then to have a method which actively releases the chemical at the appropriate point in time. If an impermeant microsphere/nanocapsule is used to house the chemical, then it is possible to selectively burst it by laser photolysis (Sun and Chiu, 2004). A third method that has been used to locally apply chemicals is to coat beads with chemicals such as pheromones, and to press the beads up against cells in order to activate ligand-receptor responses (Wei et al., 1999).

In this study we have described an optical tweezer system with a single trap that can be readily use as an experimental tool in combination with live-cell imaging. We have recently developed a two-trap system which we are also using successfully to manipulate filamentous fungi (unpublished). In addition, we have also developed an entirely different tweezer system using holographical optical trapping (Lafong et al., 2006) that involves using computer-generated holograms to create three-dimensional configurations of single-beam optical traps (Grier and Roichman, 2006). Using this approach we have been able to trap and move $<6$ yeast cells simultaneously (Lafong et al., 2006).

It is clear from the present study, from other biological applications of optical tweezers that have been reported, and from recent developments in tweezer technology, that optical tweezers will become increasingly important in livecell studies to address novel questions in fungal biology.

\section{Acknowledgments}

We thank Chris Hunt for providing the porous beads, Mike MacDonald for permission to use and adapt the diagram shown in Fig. 1 and Laurence Wilson for his advice on the manuscript. Thanks are also due to the Scottish Higher Education Funding Council for funding and the Engineering and Physical Sciences Funding Council for providing GDW with a research studentship.

\section{References}

Arneborg, N., Siegumfeldt, H., Andersen, G.H., Nissen, P., Daria, V.R., Rodrigo, P.J., Glückstad, J., 2005. Interactive optical trapping shows that confinement is a determinant of growth in a mixed yeast culture. FEMS Microbiol. Lett. 245, 155-159.

Ashkin, A., 1991. The study of cells by optical trapping and manipulation of living cells using infrared laser beams. ASGSB. Bull. 4, 133-146.

Ashkin, A., 1997. Optical trapping and manipulation of neutral particles using lasers. Proc. Natl. Acad. Sci. USA 94, 4853-4860.

Ashkin, A., Dziedzic, J.M., Bjorkholm, J.E., Chu, S., 1986. Observation of a single-beam gradient force optical trap for dielectric particles. Opt. Lett. 11, 288-290.

Bartnicki-Garcia, S., 2002. Hyphal tip growth: outstanding questions. In: Osiewacz, H.D. (Ed.), Molecular Biology of Fungal Development. Marcel Dekker, New York, pp. 29-58.
Baumann, C.G., Bloomfield, V.A., Smith, S.B., Bustamante, C., Wang, M.D., Block, S.M., 2000. Stretching of single collapsed DNA molecules. Biophys. J. 78, 1965-1978.

Bencina, M., Legisa, M., Read, N.D., 2005. Cross-talk between cAMP and calcium signalling in Aspergillus niger. Mol. Microbiol. 56, 268-281.

Berns, M.W., Wright, W.H., Wiegand, S.R., 1991. Laser microbeam as a tool in cell biology. Int. Rev. Cytol. 129, 1-44.

Berns, M.W., Aist, J.R., Wright, W.H., Liang, H., 1992. Optical trapping in animal and fungal cells using a tunable, near-infrared titanium-sapphire laser. Exp. Cell Res. 198, 375-378.

Block, S.M., 1990. Optical tweezers: a new tool for biophysics. In: Foster, G.A., Gross, S.P. (Eds.), Noninvasive Techniques in Cell Biology. Wiley-Liss, New York, pp. 375-402.

Block, S.M., 1992. Making light work with optical tweezers. Nature 360, 493-495.

Block, S.M., Blair, D.F., Berg, H.C., 1989. Compliance of bacterial flagella measured with optical tweezers. Nature 338, 514-517.

Bracker, C.E., Murphy, D.J., Lopez-Franco, R., 1997. Laser microbeam manipulation of cell morphogenesis in growing fungal hyphae. Proc. SPIE 2983, 67-80.

Cluzel, P., Lebrun, A., Heller, C., Lavery, R., Viovy, J.L., Chatenay, D., Caron, F., 1996. DNA: an extensible molecule. Science 271, 792-794.

Daria, V.R., Rodrigo, P.J., Glückstad, J., 2004. Dynamic formation of optically trapped microstructure arrays for biosensor applications. Biosens. Bioelectron. 19, 1439-1444.

Davis, R.H., 2000. Neurospora: Contributions of a Model Organism. Oxford University Press, Oxford.

Dholakia, K., Spalding, G.C., MacDonald, M.P., 2002. Optical tweezers: the next generation. Phys. World 15, 31-35.

Erlicher, A., Betz, T., Stuhrmann, B., Koch, D., Milner, V., Raizen, M.G., Kas, J., 2002. Guiding neuronal growth with light. Proc. Natl. Acad. Sci. USA 99, $16024-16028$.

Finer, J.T., Simmons, R.M., Spudich, J.A., 1994. Single myosin molecule mechanics: piconewton forces and nanometre steps. Nature 368,113 118.

Fleißner, A., Sarkar, S., Jacobson, D.J., Roca, M.G., Read, N.D., Glass, N.L., 2005. The so locus is required for vegetative cell fusion and postfertilization events in Neurospora crassa. Eukaryot. Cell 4, 920-930.

Froehlich, A.C., Noh, B., Vierstra, R.D., Loros, J., Dunlap, J.C., 2005. Genetic and molecular analysis of phytochromes from the filamentous fungus Neurospora crassa. Eukaryot. Cell 4, 2140-2152.

Galagan, J.E., Calvo, S.E., Borkovich, K.A., Selker, E.U., Read, N.D., Jaffe, D., FitzHugh, W., Ma, LJ., Smirnov, S., Purcell, S., Rehman, B., Elkins, T., Engels, R., Wang, S., Nielsen, C.B., Butler, J., Endrizzi, M., Qui, D., Ianakiev, P., Bell-Pedersen, D., Nelson, M.A., Werner-Washburne, M., Selitrennikoff, C.P., Kinsey, J.A., Braun, E.L., Zelter, A., Schulte, U., Kothe, G.O., Jedd, G., Mewes, W., Staben, C., Marcotte, E., Greenberg, D., Roy, A., Foley, K., Naylor, J., Stange-Thomann, N., Barrett, R., Gnerre, S., Kamal, M., Kamvysselis, M., Mauceli, E., Bielke, C., Rudd, S., Frishman, D., Krystofova, S., Rasmussen, C., Metzenberg, R.L., Perkins, D.D., Kroken, S., Cogoni, C., Macino, G., Catcheside, D., Li, W., Pratt, R.J., Osmani, S.A., DeSouza, C.P., Glass, L., Orbach, M.J., Berglund, J.A., Voelker, R., Yarden, O., Plamann, M., Seiler, S., Dunlap, J., Radford, A., Aramayo, R., Natvig, D.O., Alex, L.A., Mannhaupt, G., Ebbole, D.J., Freitag, M., Paulsen, I., Sachs, M.S., Lander, E.S., Nusbaum, C., Birren, B., 2003. The genome sequence of the filamentous fungus Neurospora crassa. Nature 422, 859-868.

Girbardt, M., 1957. Der Spitzenkorper von Polystictus versicolor (L.). Planta 50, 47-50.

Goksor, M., Enger, J., Hanstorp, D., 2004. Optical manipulation in combination with multiphoton microscopy for single-cell studies. Appl. Opt. 43, $4831-4837$.

Gow, N.A., 1994. Growth and guidance of the fungal hypha. Microbiology 140, 3193-3205.

Gow, N.A., Perera, T.H., Sherwood-Higham, J., Gooday, G.W., Gregory, D.W., Marshall, D., 1994. Investigation of touch-sensitive responses by hyphae of the human pathogenic fungus Candida albicans. Scanning Microsc. 8, 705-710. 
Greulich, K.O., 1999. Micromanipulation by Light in Biology and Medicine: the Laser Microbeam and Optical tweezers. Birkhauser Verlag, Basel, Switzerland.

Greulich, K.O., 2005. Single-molecule studies on DNA and RNA. Chemphyschem 6,2458-2471.

Greulich, K.O., Pilarczyk, G., 1998. Laser tweezers and optical microsurgery in cellular and molecular biology. Working principles and selected applications. Cell Mol. Biol. 44, 701-710.

Greulich, K.O., Pilarczyk, G., Hoffmann, A., Meyer Zu, H.G., Schafer, B., Uhl, V., Monajembashi, S., 2000. Micromanipulation by laser microbeam and optical tweezers: from plant cells to single molecules. J. Microsc. 198, 182-187.

Grier, D.G., Roichman, Y., 2006. Holographic optical trapping. Appl. Opt. $45,880-887$.

Grimbergen, J.A., Visscher, K., Gomes De Mesquita, D.S., Brakenhoff, G.J., 1993. Isolation of single yeast cells by optical trapping. Yeast 9 , 723-732.

Harris, S.D., Read, N.D., Roberson, R.W., Shaw, B., Seiler, S., Plamann, M., Momany, C., 2005. Polarisome meets Spitzenkorper: microscopy, genetics, and genomics converge. Eukaryot. Cell 4, 225-229.

Hickey, P.C., Swift, S.R., Roca, M.G., Read, N.D., 2005. Live-cell imaging of filamentous fungi using vital fluorescent dyes and confocal microscopy. In: Savidge, T., Pothoulakis, C. (Eds.), Microbial Imaging. In: Methods in Microbiology, vol. 35. Elsevier, London, pp. 63-87.

Hoch, H.C., Staples, R.C., Whitehead, B., Comeau, J., Wolf, E.D., 1987. Signaling for growth orientation and cell differentiation by surface topography in Uromyces. Science 235, 1659-1662.

Jedd, G., Chua, N.-H., 2000. A new self-assembled peroxisomal vesicle required for efficient resealing of the plasma membrane. Nature Cell Biol. 2, 226-231.

Krylov, S.N., Dovichi, N.J., 2000. Single-cell analysis using capillary electrophoresis: influence of surface support properties on cell injection into the capillary. Electrophoresis 21, 767-773.

Kuo, K., Hoch, H.C., 1996. Germination of Phyllosticta ampelicida pycnidiospores: prerequisite of adhesion to the substratum and the relationship of substratum wettability. Fungal Genet. Biol. 20, $18-29$.

Kuo, S.C., Sheetz, M.P., 1992. Optical tweezers in cell biology. Trends Cell Biol. 2, 116-118.

Kuo, S.C., Sheetz, M.P., 1993. Force of single kinesin molecules measured with optical tweezers. Science 260, 232-234.

Kwon, Y.H., Hoch, H.C., Aist, J.R., 1991. Initiation of appressorium formation in Uromyces appendiculatus: organization of the apex, and the responses involving microtubules and apical vesicles. Can. J. Bot. 69 , $2560-2573$.

Lafong, A., Hossack, W.J., Arlt, J., Nowakowski, T.J., Read, N.D., 2006. Time-multiplexed Laguerre-Gaussian holographic optical tweezers for biological applications. Opt. Express 14, 3065-3072.

Lang, M.J., Fordyce, P.M., Block, S.M., 2003. Combined optical trapping and single-molecule fluorescence. J. Biol. 2, 6.

Lee, I.H., Plamann, M., 2001. Microtubules and molecular motors. In: Howard, R.J., Gow, N.A.R. (Eds.), The Mycota. In: Biology of the Fungal Cell, vol. VIII. Springer-Verlag, Berlin, pp. 225-241.

Leitz, G., Schnepf, E., Greulich, K.O., 1995. Micromanipulation of statoliths in gravity-sensing Chara rhizoids by optical tweezers. Planta 197 278-288.

Leitz, G., Lundberg, C., Fallman, E., Axner, O., Sellstedt, A., 2003. Laserbased micromanipulation for separation and identification of individual Frankia vesicles. FEMS Microbiol. Lett. 224, 97-100

Lew, R.R., 2005. Mass flow and pressure-driven hyphal extension in $\mathrm{Neu}$ rospora crassa. Microbiology 151, 2685-2692.

Liang, H., Vu, K.T., Krishnan, P., Trang, T.C., Shin, D., Kimel, S., Berns, M.W., 1996. Wavelength dependence of cell cloning efficiency after optical trapping. Biophys. J. 70, 1529-1533.

Liu, X., Wang, H., Li, Y., Tang, Y., Liu, Y., Hu, X., Jia, P., Ying, K., Feng, Q., Guan, J., Jin, C., Zhang, L., Lou, L., Zhou, Z., Han, B., 2004. Preparation of single rice chromosome for construction of a
DNA library using a laser microbeam trap. J. Biotechnol. 109, $217-$ 226.

Lopez-Franco, R., Bracker, C.E., 1996. Diversity and dynamics of the Spitzenkörper in growing hyphal tips of higher fungi. Protoplasma $195,90-111$

Lucas, J.A., 2004. Survival, surfaces and susceptibility-the sensory biology of pathogens. Plant Pathol. 53, 679-691.

Markham, P., Collinge, A.J., 1987. Woronin bodies of filamentous fungi. FEMS Microbiol. Rev. 46, 1-11.

Molloy, J.E., Padgett, M.J., 2002. Lights, action: optical tweezers. Contemp. Phys. 43, 241-258.

Money, N.P., Davis, C.M., Ravishankar, J.P., 2004. Biomechanical evidence for convergent evolution of the invasive growth process among fungi and oomycete water molds. Fungal Genet. Biol. 41, 872-876.

Nelson, G., Kozlova-Zwinderman, O., Collis, A.J., Knight, M.R., Fincham, J.R., Stanger, C.P., Renwick, A., Hessing, J.G., Punt, P.J., van den Hondel, C.A., Read, N.D., 2004. Calcium measurement in living filamentous fungi expressing codon-optimized aequorin. Mol. Microbiol. $52,1437-1450$.

Neuman, K.C., Block, S.M., 2006. Optical trapping. Rev. Sci. Instrum. 75, 2787-2809.

Neuman, K.C., Chadd, E.H., Liou, G.F., Bergman, K., Block, S.M., 1999. Characterization of photodamage to Escherichia coli in optical traps. Biophys. J. 77, 2856-2863.

Read, N.D., 1991. Low-temperature scanning electron microscopy of fungi and fungus-plant interactions. In: Mendgen, K., Lesemann, D.-E. (Eds.), Electron Microscopy of Plant Pathogens. Springer-Verlag, Berlin, pp. 17-29.

Read, N.D., Kellock, L.J., Knight, H., Trewavas, A.J., 1992. Contact sensing during infection by fungal pathogens. In: Callow, J.A., Green, J.R. (Eds.), Perspectives in Plant Cell Recognition. Cambridge University Press, Cambridge, pp. 137-172.

Read, N.D., Kellock, L.J., Collins, T., Gundlach, A.M., 1997. Role of touch sensing for infection structure differentiation in cereal rust fungi. Planta 202, 163-170.

Riquelme, M., Roberson, R.W., McDaniel, D.P., Bartnicki-Garcia, S., 2002. The effects of ropy- 1 mutation on cytoplasmic organization and intracellular motility in mature hyphae of Neurospora crassa. Fungal Genet. Biol. 37, 171-179.

Roca, M.G., Arlt, J., Jeffree, C.E., Read, N.D., 2005. Cell biology of conidial anastomosis tubes in Neurospora crassa. Eukaryot. Cell 4, 911-919.

Sacconi, L., Tolić-Norrelykke, I.M., Stringari, C., Antolini, R., Pavone F.S., 2005. Optical micromanipulations inside yeast cells. Appl. Opt. 44 2001-2007.

Sheetz, M.P., 1998. Laser tweezers in cell biology. Methods Cell Biol., 55.

Spector, I., Shochet, N.R., Kashman, Y., Groweiss, A., 1983. Latrunculins: novel marine toxins that disrupt microfilament organization in cultured cells. Science 219, 493-495.

Steinberg, G., 2000. The cellular roles of molecular motors in fungi. Trends Microbiol. 8, 162-168.

Sun, B., Chiu, D.T., 2004. Synthesis, loading, and application of individual nanocapsules for probing single-cell signaling. Langmuir 20, 4614 4620.

Svoboda, K., Block, S.M., 1994. Biological applications of optical forces. Annu. Rev. Biophys. Biomol. Struct. 23, 247-285.

Tolić-Norrelykke, I.M., Munteanu, E.L., Thon, G., Oddershede, L., BergSorensen, K., 2004. Anomalous diffusion in living yeast cells. Phys. Rev. Lett. 93, 078102 .

Tolić-Norrelykke, I.M., Sacconi, L., Stringari, C., Raabe, I., Pavone, F.S., 2005. Nuclear and division-plane positioning revealed by optica micromanipulation. Curr. Biol. 15, 1212-1216.

Wang, M.D., Yin, H., Landick, R., Gelles, J., Block, S.M., 1997. Stretching DNA with optical tweezers. Biophys. J. 72, 1335-1346.

Wang, Y., Botvinick, E.L., Zhao, Y., Berns, M.W., Usami, S., Tsien, R.Y., Chien, S., 2005. Visualizing the mechanical activation of Src. Nature 434, 1040-1045. 
Weber, G., Greulich, K.O., 1992. Manipulation of cells, organelles, and genomes by laser microbeam and optical trap. Int. Rev. Cytol. 133, $1-41$.

Wei, X., Tromberg, B.J., Cahalan, M.D., 1999. Mapping the sensitivity of T cells with an optical trap: polarity and minimal number of receptors for $\mathrm{Ca}^{2+}$ signaling. Proc. Natl. Acad. Sci. USA 96, 8471-8476.
Wright, W.H., Sonek, G.J., Tadir, Y., Berns, M.W., 1990. Laser trapping in cell biology. J. Quant. Electron. 26, 2148-2157.

Wright, G.D., Arlt, J., Poon, W.C.K., Read, N.D., 2005. Measuring fungal growth forces with optical tweezers. Proc. SPIE 5930, F1-F7.

Xiang, X., Plamann, M., 2003. Cytoskeleton and motor proteins in filamentous fungi. Curr. Opin. Microbiol. 6, 628-633. 


\section{Experimentally manipulating fungi with optical tweezers}

\begin{abstract}
A short review of the use of optical tweezers in fungal cell biological research is provided. First, we describe how optical tweezers work. Second, we review how they have been used in various experimental live-cell studies to manipulate intracellular organelles, hyphal growth and branching, and whole cells. Third, we indicate how optically trapped microbeads can be used for the localized delivery of chemicals or mechanical stimulation to cells, as well as permitting measurements of the growth forces generated by germ tubes. Finally, the effects of optical trapping on fungal cell viability and growth are assessed.
\end{abstract}

Key words Germ tubes $\cdot$ Hyphal tip growth $\cdot$ Neurospora crassa $\cdot$ Optical tweezers $\cdot$ Spitzenkörper

\section{Introduction}

Optical tweezers (also termed laser tweezers or optical traps) allow microscopic particles, living cells, and organelles to be trapped and manipulated with light. Since their invention 20 years ago (Ashkin et al. 1986), optical tweezers

G.D. Wright $\cdot$ N.D. Read $(\bowtie)$

Fungal Cell Biology Group, Institute of Cell Biology, University of Edinburgh, Rutherford Building, Edinburgh EH9 3JH, UK

Tel. +44-131-650-5335; Fax +44-131-650-5392

e-mail: Nick@fungalcell.org

G.D. Wright - J. Arlt ${ }^{1}$ - W.C.K. Poon - N.D. Read

Collaborative Optical Spectroscopy, Micromanipulation and Imaging Centre (COSMIC), University of Edinburgh, Edinburgh, UK

J. Arlt · W.C.K. Poon

SUPA and School of Physics, University of Edinburgh, Edinburgh, UK

\section{Present address:}

${ }^{1}$ Department of Physics, JJ Thomson Physical Laboratory, The University of Reading, Reading, UK

Parts of this review were presented at the Mycological Society of Japan (MSJ) / British Mycological Society (BMS) Joint Symposium, "The new generation mycologists in Japan and the UK" held in Chiba, Japan on June 3, 2006. have been employed in a wide variety of biological research applications (Block 1990; Kuo and Sheetz 1992; Ashkin 1997; Sheetz 1998; Greulich 1999; Molloy and Padgett 2002; Neuman and Block 2006). So far, however, optical tweezers have been little used as experimental tools in fungal cell biology (Wright et al. 2007). In this short review we provide a summary of work in this area.

\section{How optical tweezers work}

Optical tweezers facilitate the noninvasive micromanipulation of both inert and biological microscopic particles solely by using light. They utilize an intense laser light source, which is tightly focused by a microscope objective lens of high numerical aperture to produce a three-dimensional optical trap. When photons are absorbed, reflected, or refracted by a transparent object, the momentum they possess is changed, which corresponds to an action force acting on these photons. Newton's third law states that for every action force there is a corresponding reaction force that is equal in magnitude and opposite in direction; therefore, the object creating the action force will have the reaction force applied to it (Fig. 1). With a laser beam of Gaussian profile, the net force experienced by a spherical object is zero at a point close to the focus of the laser beam. This point is then the center of the optical trap. When the object is displaced slightly from this trap center, it experiences a restoring force that is proportional to the displacement, up to a certain maximum, after which the object escapes. The trapping forces generated are typically of the order of piconewtons and sufficient to trap and move microscopic objects up to tens of micrometers in size. For an object to be trapped efficiently, it must have a refractive index that is sufficiently higher than its surroundings (Fig. 2). Tweezer systems can be equipped with multiple traps either by using more than one laser or by generating holographic optical tweezers with a computer-controlled spatial light modulator from a single laser source (Grier and Roichman 2006). 


\section{Methodology}

We have built a simple, compact, easy-to-use, safe, and robust dual-trap optical tweezer system that can be used with brightfield, phase-contrast, differential interference contrast, and fluorescence optics. This apparatus has been mounted on both Nikon TE2000U and Olympus IX70 inverted light microscopes and is an upgraded version of the one-trap tweezer system that we have described previously (Wright et al. 2007). In our latest tweezer system, the location of onc trap in the field of view is computer-controlled using galvanometric mirrors, allowing easy and accurate positioning of trapped objects with the click of a mouse (Wright et al. 2007). The other trap is fixed in the center of the field of view and requires the microscope stage to be moved to change the position of a trapped object. Both lasers are 785-nm diode lasers with a maximum output power of $70 \mathrm{~mW}$.

Our experimental systems are conidia, conidial germlings, and vegetative hyphae of Neurospora crassa. Filamentous fungi are well suited to being micromanipulated by optical tweezers because they can be analyzed as a monolayer of cells or hyphae, which are sometimes very large, and can exhibit very rapid growth responses to physical and chemical stimuli (Wright et al. 2007).

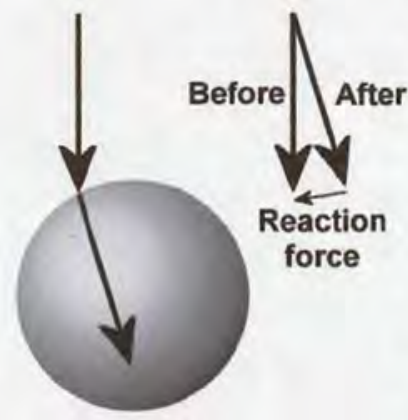

Fig. 1. A ray diagram depicting the interaction of a single ray of light with a transparent sphere. The sphere refracts the light changing its direction and therefore momentum (an action force). The resultant reaction force is opposite in direction and equal in magnitude (Newton's third law). By focusing a laser beam of Gaussian profile through a microscope objective lens of high numerical aperture, the net reaction force is to trap the object at the region of highest light intensity, the point of focus
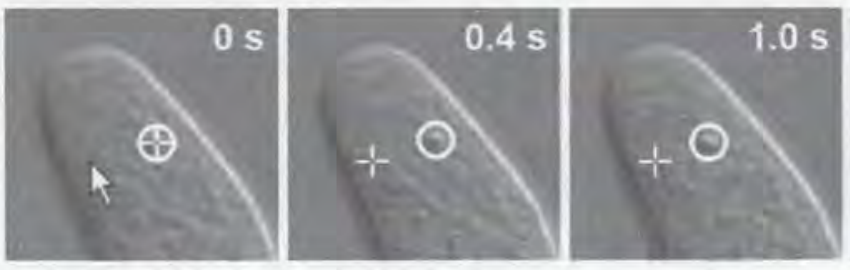

Fig. 2. Differential interference contrast microscopy showing the trapping and moving of a spherical organelle of high refractive index within a hypha of Neurospora crassa. The sequence shows the organelle being
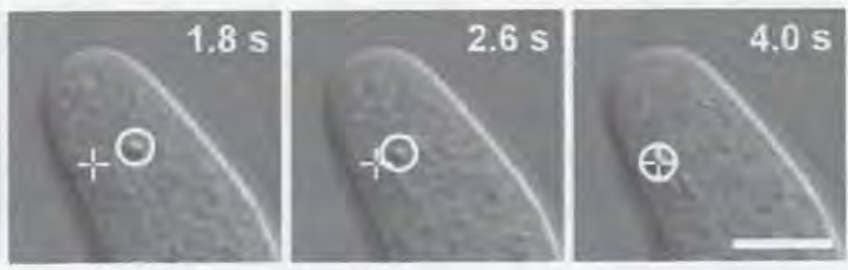

Manipulating organelles of high and low refractive index

Organelles that are of higher refractive index than their surrounding cytoplasm can be trapped with optical tweezers. Good examples of this are the highly refractile Woronin bodies that are involved in septal pore occlusion (Berns et al. 1992; Wright et al. 2007; Wright et al. 2007: supplementary movie 3: www.fungalcell.org/tweezermovies.htm). Once trapped, organelles can usually be easily moved as long as they are not strongly tethered in place (e.g., to cytoskeletal elements). Figure 2 shows the trapping and movement of a spherical organelle across a hypha. The cytoplasm is a highly viscous environment, and as a result moving an organelle must be done slowly to prevent it being lost from the trap. In contrast, organelles of lower refractive index, such as vacuoles, are repelled from the trap (Wright et al. 2007). Although this makes it more difficult, tweezers can still be used to micromanipulate organelles by a technique that involves "chasing" them in a manner akin to repelling magnets (Wright et al. 2007: supplementarys movies 4 and 5: www.fungalcell.org/tweezermovies.htm). It is also possible to press repelled vacuoles against the plasma membrane and split them in two (Wright et al. 2007; Wright et al. 2007: supplementary movie 6: www.fungalcell.org/tweezermovies.htm).

\section{Manipulating hyphal growth and branching}

The Spitzenkörper, the secretory vesicle-dominated organelle complex that regulates tip-growth (Bartnicki-Garcia 2002; Harris et al. 2005; Virag and Harris 2006), was previously shown to be unexpectedly repelled by optical trapping (Bracker et al. 1997; Wright et al. 2005, 2007). The reason for this dark-phase (high refractive index) structure being repelled rather than trapped is not understood, and more than one mechanism may be involved (Wright et al. 2007). The direction of tip growth can be repeatedly redirected, causing a "zigzag" pattern of hyphal growth (Fig. 3; also see Wright et al. 2007: supplementary movie 7: www.fungalcell. org/tweezermovies.htm). Alternatively, parking the optical trap at one point beneath the hyphal surface can induce branch formation, probably as a result of concentrating secretory vesicles in that location (Bracker et al. 1997).

moved laterally across the hypha. The current position of the trap is represented by the circle; the cross-hair shows the position to which the trap is being moved. Bar $5 \mu \mathrm{m}$ 

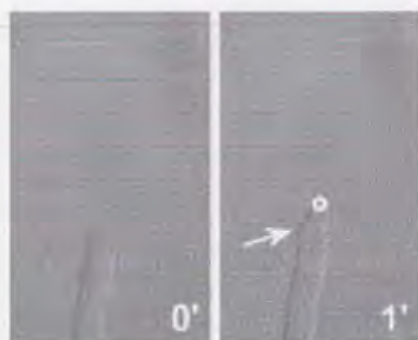

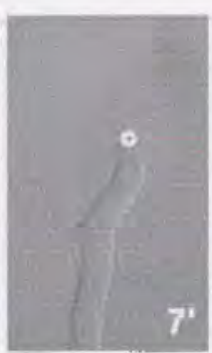

$7^{\prime}$
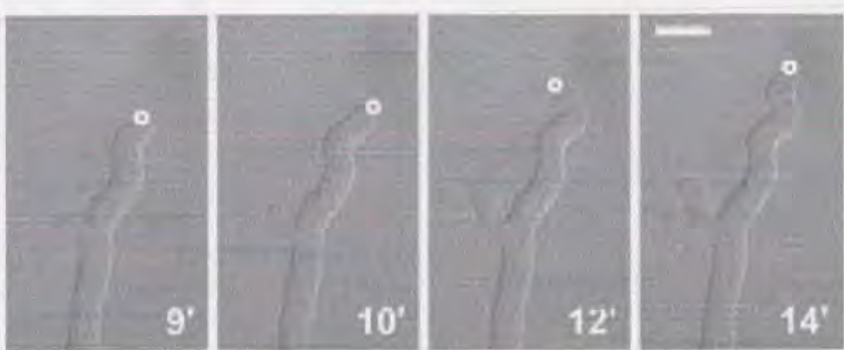

growth, giving rise to the zigzag growth pattern of the hypha. A branch was formed at the point of initial exposure to the trap (arrow at $1 \mathrm{~min}$ ). The current position of the trap is represented by the circle. Bar $10 \mu \mathrm{m}$

tion of growth by manipulating the Spitzenkörper. When the laser is reoriented away from the trap because the Spitzenkörper is repelled by it. The trap was repeatedly repositioned to repeatedly redirect tip
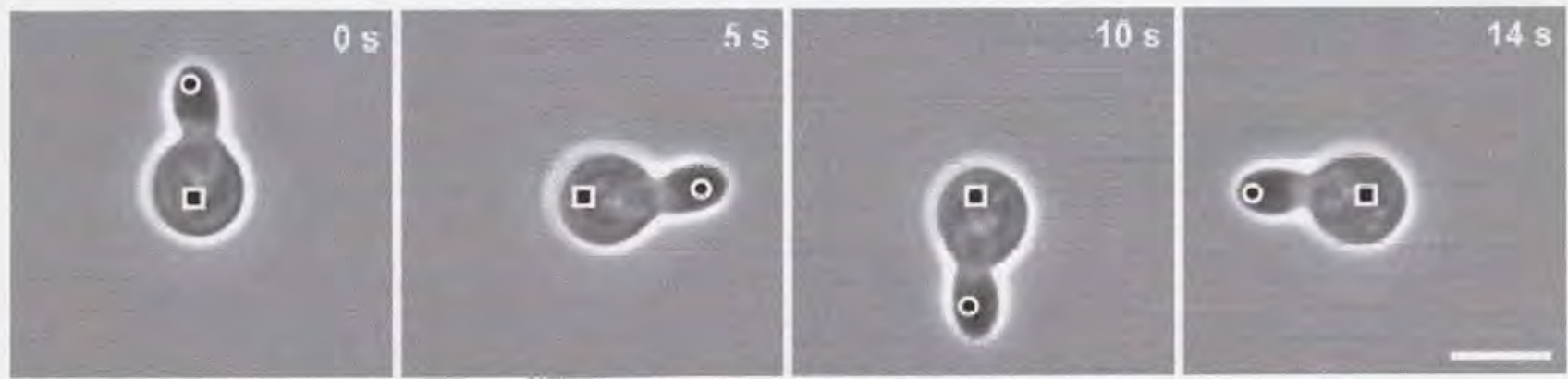

Fig. 4. Phase-contrast microscopy showing rotation of a conidial germling using two traps. The conidium is held by the stationary trap (square); the germ tube tip is held by the moveable trap (circle). Bar $5 \mu \mathrm{m}$

\section{Manipulating whole cells to study cell-cell signalling}

Whole spores or spore germlings can be trapped and moved around in liquid culturc or in a liquid film on solid medium (Wright et al. 2007; Wright et al. 2007: supplementary movies 1 and 2: www.fungalcell.org/tweezermovies.htm). Moving whole spores with optical tweezers has been used to investigate the signaling that occurs between conidial anastomosis tubes (CATs) that are homing towards each other (Fleißner et al. 2005; Roca et al. 2005). With the recent addition of a second trapping laser into our system, we are now able to trap two germinating spores simultaneously and reposition one with respect to the other; this helps overcome the problem of one of the homing spore germlings drifting away from the other over the typical 20- to 30-min period of the CAT homing assay (Fleißner et al. 2005). Another use of two traps is to enable precise control over the orientation of germinated spores. In Fig. 4, the conidium was trapped with the stationary laser whilst the emergent germ tube tip was trapped with the steerable laser.

We have developed a technique using optical tweezers to establish arrays of spores that are adhered to coverslips. By coating half the area of a coverslip with polyvinyl alcohol (PVA), a nonstick region was created (Krylov and Dovichi 2000). Ungerminated conidia were then trapped with the optical tweezers and carried from the PVA region to the uncoated area. Briefly holding the conidia against the glass caused them to adhere to it. Repeating this procedure allowed lixed arrays of spores to be generated in which the distance separating cells could be accurately defined (Fig. 5). This method provides another experimental approach to study communication between cells within a cell population (e.g., in relation the signals involved in CAT homing and germ tube avoidance; Roca et al. 2005).

Holographic optical tweezers potentially provide a more elegant way of producing microarrays of trapped cells. Here, multiple traps are generated from a single laser source, and each trap can be individually computer controlled. Lafong et al. (2006) successfully used a holographic tweezer system to trap arrays of up to six cells of Saccharomyces cerevisiae and conidia of $N$. crassa and to move the cells relative to one another.

\section{Using optically trapped microbeads as experimental tools}

Microbeads that are $1-10 \mu \mathrm{m}$ in diameter, and that have a higher refractive index than their surroundings, can be efficiently trapped with optical tweezers. Commonly, these microbeads are made of polystyrene or silica and are widely available commercially. Once trapped they can be used as tools to experimentally manipulate biological samples.

Chemicals can be delivered to localized regions of cells with microbeads by (1) soaking porous microbeads in a 


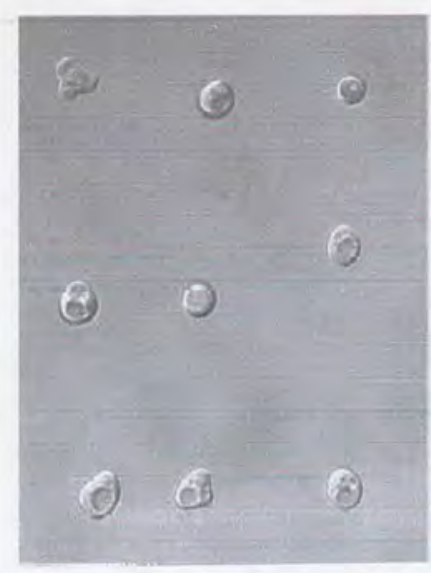

Fig. 5. Differential interference microscopy showing the production of microarrays of conidia. Macroconidia were trapped in an area of coverslip made nonsticky by coating it with polyvinyl alcohol (PVA) and then moved carefully using the microscope stage controls, up to $1 \mathrm{~cm}$
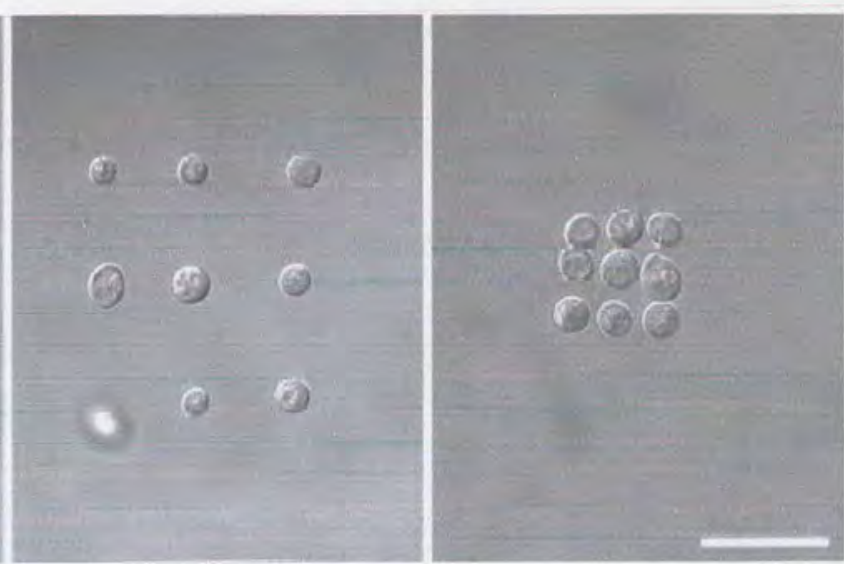

across the coverslip, to an uncoated region of the coverslip where they were carefully positioned and brought into contact with the uncoated glass to adhere. These spore arrays were stable and could be observed over long periods of time whilst germination took place. Bar $25 \mu \mathrm{m}$
Fig. 6. Differential interference microscopy showing the obstruction of germ tube growth with an optically trapped $4-\mu \mathrm{m}$ bead. Note that upon first making contact with the bead the germ tube tip moved the bead very slightly but was unable expel it from the trap; this was subsequently followed by swelling of the germ tube and cessation of its growth. When the obstacle was removed, extension of the germ tube resumed. The highest output laser power $(70 \mathrm{~mW})$ was used in this experiment, which equates to a trapping force of $19 \mathrm{pN}$ (Wright et al. 2005). Bar $10 \mu \mathrm{m}$
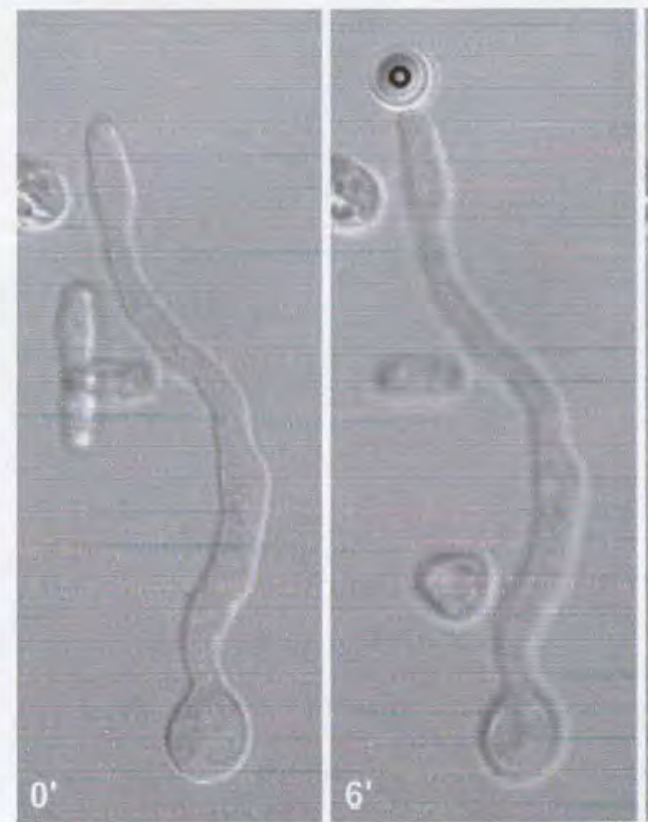

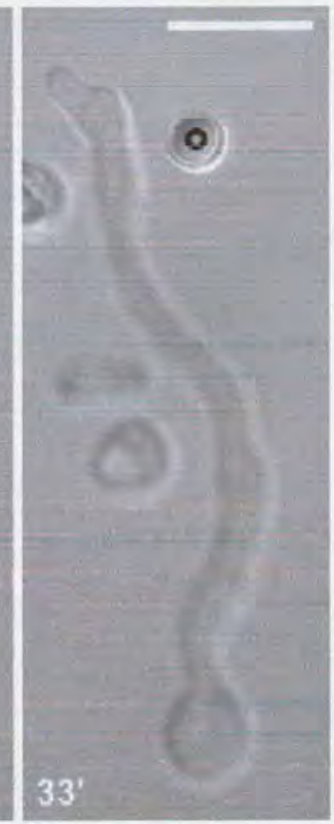

chemical solution to provide a continuous release of the chemical from the beads (Wright et al. 2007), (2) trapping a chemical in microcapsules and releasing it on demand (Sun and Chiu 2004), or (3) pressing against a cell surface trapped microbeads that have been coated with chemicals (Wei et al. 1999).

A mechanostimulus can be locally applied to cells or hyphae by hitting them with a trapped bead. Wright et al. (2007) demonstrated this using a $10-\mu \mathrm{m}$ polystyrene microbead to repeatedly hit growing vegetative hyphal tips. The effect of this treatment was to cause a slight redirection of hyphal growth without changing the rate of hyphal extension (Wright et al. 2007: supplementary movie 10: www.fungalcell.org/tweezermovies.htm).

Trapped microbeads can be used to measure growth forces produced by fungal cells. The force required to dis- place a bead from an optical trap can be calibrated (Wright et al. 2005) and hence optical tweezers can be used to measure forces applied to trapped beads (Wright et al. 2007 supplementary movie 11: www.fungalcell.org/tweezermovies. $\mathrm{htm}$ ). The maximum trapping force varies with the size of the bead and, for a given size of bead, linearly increases with the laser power. In most cases, the forces produced by leading vegetative hyphal tips are probably far in excess of those which laser tweezers can generate (Money et al. 2004; Wright et al. 2005, 2007; Wright et al. 2007: supplementary movie 8 : www.fungalcell.org/tweezermovies.htm). However, we found that a conidial germ tube will push on a trapped bead and move it slightly but not expel it from the trap. If the trap is maintained in position, the germ tube subsequently swells and stops growing (Fig. 6; Wright et al. 2007: supplementary movie 9: www.fungalcell.org/ 
tweezermovies.htm). These results indicated that germ tubes generate significantly smaller growth forces than leading vegetative hyphae (Wright et al. 2006).

\section{Effects of optical tweezers on cell viability}

Optical tweezers are often described as a "non-invasive" technique for studies with living cells. However, significant photodamage can occur if the user chooses a suboptimal combination of laser wavelength, laser power, and duration of exposure to the laser beam, as well as taking into account the type of biological sample being irradiated (Liang et al. 1996; Neuman et al. 1999). Wavelengths that have been successfully used as optical traps in studies on living fungal cells are $647 \mathrm{~nm}$ (Lafong et al. 2006), 760 nm (Berns et al. 1992), $785 \mathrm{~nm}$ (Fleißner et al. 2005; Roca et al. 2005; Wright et al. 2005, 2007; also this article), $820 \mathrm{~nm}$ (Bracker et al. 1997), and $830 \mathrm{~nm}$ (Arneborg et al. 2005; Sacconi et al. 2005). We found that a 30 -s exposure of ungerminated conidia of $N$. crassa to a 785-nm trapping laser had no signilicant effect on the onset of conidial germination, the rate of germination, or the final percentage of conidia that germinated (Wright et al. 2007). Furthermore, conidial germlings continuously optically trapped for $25 \mathrm{~min}$ exhibited no deleterious effects with regard to conidial anastomosis tube growth, homing, or fusion (Wright et al. 2007). Yeast cells of $S$. cerevisiae were found not to be substantially heated by trapping them with optically tweezers at $830 \mathrm{~nm}$. Potential heating of these cells was assessed by monitoring spindle pole elongation, which is a temperature-sensitive process (Sacconi et al. 2005).

\section{Conclusions}

We have used optical tweezers in a range of fungal cell biology applications to trap and micromanipulate whole fungal cells, organelles, and beads. Optical tweezers are very powerful experimental tools that will have numerous applications in mycological research in the future. They will be particularly important when integrated with the analysis of living cells using advanced imaging techniques.

Acknowledgments We thank the Scottish Higher Education for funding and the Engineering and Physical Sciences Funding Council for providing G.D.W. with a research studentship. Also, thanks are due to Andrew Grant, who designed and built our dual-trap tweezer systems, and to Tom Nowakowski for developing methods to selectively adhere conidia to coverslips.

\section{References}

Arneborg N, Siegumfeldt H, Andersen GH, Nissen P, Daria VR, Rodrigo PJ, Glückstad J (2005) Interactive optical trapping shows that confinement is a determinant of growth in a mixed yeast culture. FEMS Microbiol Lett 245:155-159

Ashkin A (1997) Optical trapping and manipulation of neutral particles using lasers. Proc Natl Acad Sci USA 94:4853-4860

Ashkin A, Dziedzic JM, Bjorkholm JE, Chu S (1986) Observation of a single-beam gradient force optical trap for dielectric particles. Opt Lett 11:288-290

Bartnicki-Garcia S (2002) Hyphal tip growth: outstanding questions. In: Osiewacz HD (ed) Molecular biology of fungal development. Dekker, New York, pp 29-58

Berns MW, Aist JR. Wright WH, Liang H (1992) Optical trapping in animal and fungal cells using a tunable, near-infrared titanium-sapphire laser. Exp Cell Res 198:375-378

Block SM (1990) Optical tweezers: a new tool for biophysics. In: Foster GA, Gross SP (eds) Noninvasive techniques in cell biology. Wiley, New York, pp 375-402

Bracker CE, Murphy DJ, Lopez-Franco R (1997) Laser microbeam manipulation of cell morphogenesis in growing fungal hyphae. Proc SPIE 2983:67-80

Fleißner A, Sarkar S, Jacobson DJ, Roca MG, Read ND, Glass NL (2005) The so locus is required for vegetative cell fusion and postfertilization events in Neurospora crassa. Eukaryot Cell 4:920-930

Greulich KO (1999) Micromanipulation by light in biology and medicine: the laser microbeam and optical tweezers. Birkhauser, Basel

Grier DG, Roichman Y (2006) Holographic optical trapping. Appl Opt 45:880-887

Harris SD, Read ND, Roberson RW, Shaw B, Seiler S, Plamann M, Momany C (2005) Polarisome meets Spitzenkörper: microscopy, genetics, and genomics converge. Eukaryot Cell 4:225-229

Krylov SN, Dovichi NJ (2000) Single-cell analysis using capillary electrophoresis: influence of surface support properties on cell injection into the capillary. Electrophoresis 21:767-773

Kuo SC, Sheetz MP (1992) Optical tweezers in cell biology. Trends Cell Biol 2:116-118

Lafong A, Hossack WJ, Arlt J, Nowakowski TJ, Read ND (2006) Time-multiplexed Laguerre-Gaussian holographic optical tweezers for biological applications. Opt Express 14:3065-3072

Liang H, Vu KT, Krishnan P, Trang TC, Shin D, Kime, S, Berns MW (1996) Wavelength dependence of cell cloning efficiency after optical trapping. Biophys J 70:1529-1533

Molloy JE, Padgett MJ (2002) Lights, action: optical tweezers. Contemp Phys 43:241-258

Money NP, Davis CM, Ravishankar JP (2004) Biomechanical evidence for convergent evolution of the invasive growth process among fungi and oomycete water molds. Fungal Genet Biol 41:872-876

Neuman KC, Block SM (2006) Optical trapping. Rev Sci Instrum 75:2787-2809

Neuman KC, Chadd EH, Liou GF, Bergman K, Block SM (1999) Characterization of photodamage to Escherichia coli in optical traps. Biophys J 77:2856-2863

Roca MG, Arlt J, Jeffree CE, Read ND (2005) Cell biology of conidial anastomosis tubes in Neurospora crassa. Eukaryot Cell 4:911-919

Sacconi L, Tolić-Nørrelykke IM, Stingari C, Antolini R, Pavone S (2005) Optical micromanipulations inside yeast cells. Appl Opt 44:2001-2007

Sheetz MP (1998) Laser tweezers in cell biology. In: Sheetz MP (ed) Methods in Cell Biology, vol. 55, Academic, San Diego, pp xi-xii

Sun B, Chiu DT (2004) Synthesis, loading, and application of individual nanocapsules for probing single-cell signaling. Langmuir 20:4614-4620

Virag A, Harris SD (2006) The Spitzenkörper: a molecular perspective. Mycol Res 110:4-13

Wei X. Tromberg BJ, Cahalan MD (1999) Mapping the sensitivity of $\mathrm{T}$ cells with an optical trap: polarity and minimal number of receptors for $\mathrm{Ca}^{2+}$ signaling. Proc Natl Acad Sci USA 96:84718476

Wright GD, Arlt J, Poon WCK, Read ND (2005) Measuring fungal growth forces with optical tweezers. Proc SPIE 5930:F1-F7

Wright GD, Arlt J, Poon WCK, Read ND (2007) Optical tweezer micromanipulation of filamentous fungi. Fungal Genet Biol (in press) 


\title{
Holographic and single beam optical manipulation of hyphal growth in filamentous fungi
}

\author{
D R Burnham ${ }^{1,3,4}$, G D Wright ${ }^{2,4}$, N D Read ${ }^{2}$ and D McGloin ${ }^{1,3}$ \\ ${ }^{1}$ SUPA, School of Physics and Astronomy, University of St Andrews, North Haugh, \\ St Andrews, KY16 9SS, UK \\ ${ }^{2}$ Fungal Cell Biology Group, Institute of Cell Biology, University of Edinburgh, \\ Rutherford Building, Edinburgh EH9 3JH, UK \\ ${ }^{3}$ Electronic Engineering and Physics Division, Nethergate, University of Dundee, \\ Dundee, UK
}

E-mail: Nick@ fungalcell.org and dm11@st-and.ac.uk

Received 2 January 2007, accepted for publication 31 May 2007

Published 24 July 2007

Online at stacks.iop.org/JOptA/9/S172

\begin{abstract}
We report on the ability of holographic light fields to alter the normal growth patterns of filamentous fungi. The light fields are produced on a microscopic scale by borrowing methods from the field of optical tweezers, but without the aim of directly trapping or manipulating objects. Extended light fields are shown to redirect and constrict hyphal tip growth, and induce hyphal branching in a highly reproducible manner. The merits of using discrete and continuous light fields produced using a spatial light modulator are discussed and the use of three-dimensional 'pseudowalls' of light to control the growth patterns is reported. We also demonstrate the dependence of hyphal tip growth on the wavelength of light, finding that less power is needed at shorter wavelengths to effect changes in the growth dynamics of fungal hyphae.
\end{abstract}

Keywords: filamentous fungi, micromanipulation, optical tweezers, holography, Neurospora, hyphal tip growth

(Some figures in this article are in colour only in the electronic version)

\section{Introduction}

Light is well known to affect many biological processes, including growth, and can be used to experimentally manipulate growth at both organismal [1] and cellular levels [2]. The focusing of light through a microscope is ideal for manipulation at the cellular level and tools such as optical tweezers have proved themselves in many areas of biology.

In recent years optical tweezers have been used extensively as tools for micromanipulating biological systems. First demonstrated by Ashkin and colleagues [3] by trapping neutral microspheres, optical tweezers quickly found use in manipulating individual cells, viruses and bacteria $[4,5]$. They have been used in a wide range of applications including:

\footnotetext{
${ }^{4}$ Contributed equally.
}

micromanipulating cells to redirect their growth [6-10]; micromanipulating organelles within cells $[6,11,12]$; isolating individual cells, organelles and chromosomes [13, 14]; measuring the forces produced by motor proteins and RNA polymerases [15-18]; measuring the biophysical properties of DNA [19-24]; fusing cells [25]; providing localized mechanostimulation to cells [26]; and automated cell sorting [27].

It is important to note that it is not the ability to trap and manipulate objects that is of interest here but the potential of optical tweezer techniques to precisely position a highly localized laser intensity gradient.

Whilst optical tweezers have been widely used in biological investigations, their use with filamentous fungi has been largely neglected. The filamentous fungi are an extremely important group of organisms (e.g. in causing 


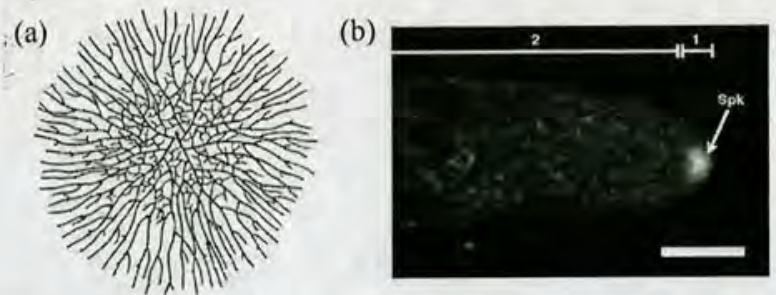

Figure 1. (a) The colony of a filamentous fungus is composed of a network of hyphae (reproduced from [37]). The hyphae studied in the present investigation are those located at the colony periphery. (b) A confocal image of a growing hyphal tip of Neurospora crasse at the colony periphery after staining with the membrane-selective fluorescent dye FM4-64 [38]. Note the concentration of stained secretory vesicles within the Spitzenkörper (Spk), the region of hyphal extension (1), and the non-extending region (2) (adapted from [39]). Bar $=5 \mu \mathrm{m}$.

human and crop diseases, food spoilage, nutrient recycling in the biosphere, promoting the growth of plants with which they have symbiotic associations, in food production, brewing and as a source of pharmaceutical drugs [28]). The colony of a filamentous fungus develops into a complex interconnected network of multinucleate, tubular cellular elements called hyphae (figure 1(a)) [29]. These hyphae grow at their tips and penetrate with force and by digestion through the microenvironments which they inhabit. Hyphae exhibit tip growth as the result of the activity of a multi-component structure called the Spitzenkörper. The main component of the Spitzenkörper are secretory vesicles and, amongst other functions, it acts as a vesicle supply centre delivering 'wall-building' secretory vesicles to a localized region (the extension zone) which results in hyphae extending exclusively at their tips (figure 1(b)) [30, 31]. Neurospora crassa, the species used throughout this investigation, is commonly used as a model system for experimental studies [32, 33] and was the first filamentous fungus to have its complete genome sequenced $[28,34]$. It produces large, fast growing hyphae making it an excellent system for combining live-cell microscopic imaging with experimental micromanipulation using optical tweezers [35, 36].

Berns et al [11] were the first to use optical tweezers with filamentous fungi, showing that organelles could be manipulated inside living hyphae without causing damage. Bracker et al [7] later used optical tweezers to change the direction of hyphal growth, concentrate secretory vesicles and induce branching. A significant observation was that the Spitzenkörper seemed to be repelled by the laser rather than being trapped, as was later confirmed by Wright et al $[35,36,39]$. Because the Spitzenkörper has a predominately higher refractive index than the adjacent cytoplasm [40], one would have expected it to be trapped under these conditions [7]. Wright et al [39] attempted to use optical tweezers to measure the growth forces generated by hyphae at the colony periphery of $N$. crassa (figure 1). The trapping forces acting upon beads were calibrated and then the beads placed in the path of the growing hyphal tips. The study concluded that these hyphae were able to generate growth forces in excess of the forces achievable with the optical trap used (i.e. $>19 \mathrm{pN}$ ). It was later shown that the specialized hyphae (called germ tubes) produced during spore germination, and which are involved in colony establishment, are not able to overcome the optically trapped beads, suggesting that they produce growth forces $<19 \mathrm{pN}$. Moreover, the germ tubes responded to the trapped beads by undergoing apical swelling until the trapped beads were removed whereupon their growth extension resumed $[35,36]$. In contrast, when mechanically stimulated with beads, the fast growing hyphae at the colony periphery (figure 1) only underwent a slight redirection in growth and their rate of extension was unaffected [35].

Another consideration of studies on irradiating fungal hyphae with light is that fungi possess a variety of photoreceptors and a range of responses to light. Little is known about lightmediated responses of fungal hyphae, except that hyphal tips have been shown to avoid $785 \mathrm{~nm}[35,36,39]$ and $830 \mathrm{~nm}$ [7] light. Whether these are photoreceptor-mediated responses, another physiological response, or some sort of physical repulsion of the Spitzenkörper by the tweezers is not yet known.

Previous studies have brought about alterations in the pattern of hyphal tip growth by what can be termed active methods; the single or dual beam conventional Gaussian tweezers have been actively moved to interfere with the hyphal tips $[7,35,36]$. However, the current work aims to use passive methods, in which the hyphae are allowed to grow toward and through stationary optical tweezers. Of significant interest would be the possibility to not only briefly redirect the hyphal tips but to manipulate hyphal growth over significant distances, and possibly into complex patterns.

To this end we note that the field of optical manipulation has proven techniques that allow light to be 'sculpted' into more complex structures than single or dual beam Gaussian tweezers achieve. Multiple traps can be created by scanning a laser beam and time sharing its focus between sites using galvo mirrors, piezo-actuated mirrors [41] or acousto-optical deflectors [42]. Another possibility is to use diffractive optical elements to split a single beam between multiple trap sites [43] which can easily become dynamic by using liquid crystal devices, such as spatial light modulators (SLMs), to display phase-only holograms [44]. These holographic optical tweezers (HOTs) [45] have become impressive tools [46, 47] and can be used in a time sharing mode [48]. Although yeast cells and ungerminated spores have previously been manipulated with multiple optical traps [49-51], fungal hyphae, and hyphal tip growth, have not.

Clearly, SLMs are not limited to producing simple patterns of Gaussian beams. Much work has been carried out to create dynamic real time 3D holography [52]. Using a GerchbergSaxton algorithm [53] it is relatively simple to create twodimensional continuous patterns of light $[54,55]$. With regard to manipulating the direction of growth of fungal hyphae by optical repulsion, as opposed to simply manipulating them, these multi-spot and continuous light pattern capabilities are ideal and offer opportunities for novel experimentation.

By borrowing the light sculpting techniques of optical tweezers, but without the sole purpose of trapping, the main aims of this study were to further assess what processes give rise to the redirection of hyphal growth by light, and to determine how the pattern of hyphal growth and branching can be manipulated over extended distances with HOTs. 


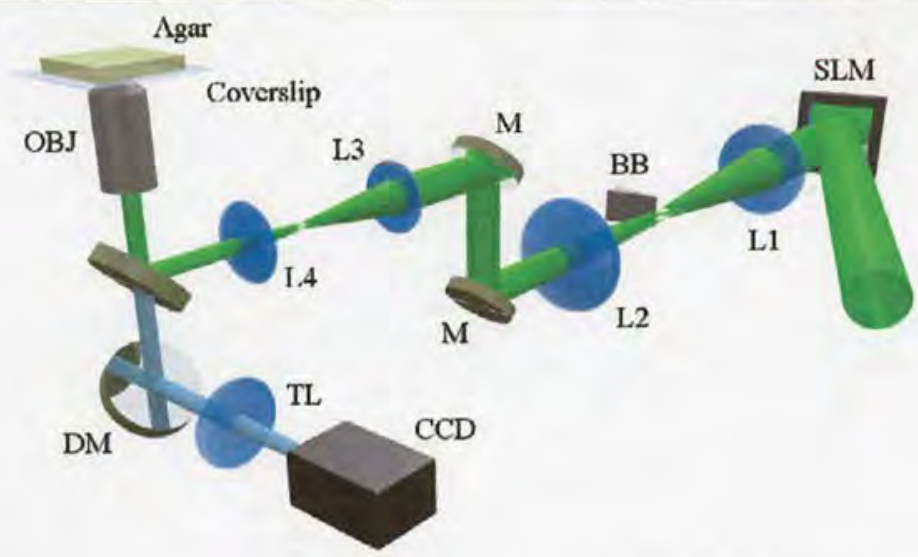

Figure 2. Schematic showing the holographic optical tweezers apparatus. Lenses $L 1$ and $L 2$ and $L 3$ and $L 4$ form two $4 f$ imaging systems, placing the objective back aperture conjugate to the SLM. BB is a beam block to remove the unwanted zeroth diffracted order. OBJ is either a Nikon Plan or a Nikon E Plan $100 \times 1.25$ NA oil microscope objective. DM is a dichroic mirror, $\mathrm{M}$ are dielectric mirrors and TL is a tube lens. The sample is illuminated with custom built Köhler illumination (not shown).

\section{Experimental details}

\subsection{Optical tweezer setup(s)}

Three different optical tweezers setups were used, each with a different wavelength of light. That using $785 \mathrm{~nm}$ light has been previously described [35]. The $1064 \mathrm{~nm}$ system involved a similar standard optical tweezing setup [56]. The $532 \mathrm{~nm}$ experiments were carried out mainly with HOTs, described in section 2.2, but for single tweezers the SLM was replaced by a mirror.

\subsection{Holographic optical tweezers system}

The holographic optical tweezers apparatus is shown in figure 2. The diffraction efficiency of a Holoeye LC-R 2500 spatial light modulator is optimized by rotating a linearly polarized beam from a Laser Quantum Finesse $532 \mathrm{~nm}$ laser with a half-wave plate. The beam is then expanded to entirely fill the width of the phase modulating device. Two $4 \mathrm{f}$ imaging systems are designed and positioned such that the SLM is conjugate to the back aperture of either a Nikon Plan (objective A) or a Nikon E Plan (objective B), $100 \times 1.25 \mathrm{NA}$ oil microscope objective whilst still slightly overfilling the aperture. The objective used for the results presented here is indicated in each figurc. The same objective, in conjunction with custom built Köhler illumination and a $200 \mathrm{~mm}$ tube lens, formed an image on a Basler A602f Firewire CCD camera.

\subsection{Hologram generation}

To generate the phase-only holograms, or kinoforms, whose optical Fourier transform gives the desired intensity pattern in the objective's focal plane, one of two algorithms was implemented using custom written LabVIEW programs. The first is a superposition of complex functions describing gratings and lenses [44] integrated into an interactive 'point and click' [57] program. The second uses a slightly modified Gerchberg-Saxton algorithm - the adaptive-additive algorithm [58], which allows the user to input an arbitrary 8-bit greyscale image containing the desired intensity pattern whether it be single points or continuous lines.

It is important to note that SLMs are notorious for having irregularly shaped back planes. When focusing their reflected collimated beams to a diffraction limited spot it becomes obvious that a significant amount of aberration has been induced in addition to that from misalignment in the optical train alone.

By implementing another custom written LabVIEW program using adaptive optics without a wavefront sensor [59] the aberration can be quantified in terms of Zernike polynomials and coefficients [60]. This information is used to generate a correction kinoform, simply by adding $\bmod 2 \pi$ to the normal kinoform, that compensates for the system's aberrations and results in a six-fold increase in the Strehl ratio for Gaussian foci. The Strehl ratio increase was calculated from the ratio of the peak intensities after and before correction.

The continuous light patterns produced only exist with a reasonable fidelity in a relatively shallow axial (along the beam propagation direction) plane. Experiments with axially extended light fields, or 'pseudowalls' of light, were also performed. These walls consisted of multiple, twodimensional, continuous light patterns displaced above and below the normal focus. Figure 3 shows a diagram of the clear difference between these pseudowalls and the simple, axially compact structures normally produced. The kinoform to be placed on the SLM is created simply by calculating those required to produce particular patterns at desired axial planes and then finding the argument of their complex sum. The result is a single, phase-only hologram producing patterns in multiple, spatially separated, axial planes.

\subsection{Fungal strains, culture conditions and sample preparation}

The $N$. crassa wild-type strain 74-OR23-1VA (no 2489, FGSC, Kansas City, KS, USA) was used. It was grown and maintained on solid Vogel's minimal medium [32] at room temperature. 
(a)

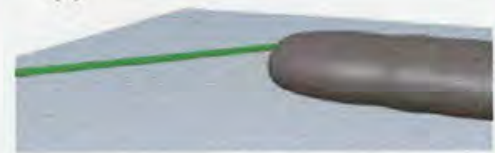

(b)

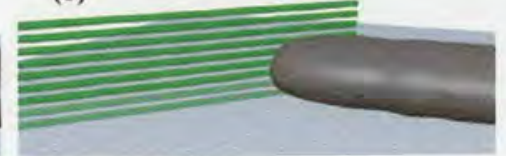

Figure 3. Diagrammatic representation of a hyphal tip approaching (a) a single line of light, as shown in the results of figures 6-9, and (b) nine, axially stacked, single lines of light, or a pseudowall, as shown in the result of figure 10.

An agar plug was used to inoculate a plate at one edge, and allowed to grow for $\sim 17 \mathrm{~h}$ before experimentation. To image and manipulate hyphal tips, the inverted agar block culture method was used [38]. Tips were given $>30 \mathrm{~min}$ to recover and resume normal growth once sample preparation was completed.

\subsection{Digital image processing}

Images were captured as movic files directly through custom written LabVIEW programs or using Hypercam screen capture software. Further processing was carried out with more custom written LabVIEW programs, Paintshop Pro (v. 8; JASC Software), Animation Shop (v. 3; JASC Software), Premier Pro (v. 1.5; Adobe) and Windows Movie Maker (v. 5.1; Microsoft).

\section{Results}

Each experiment was carried out between 2 and 15 times.

\subsection{Gaussian beams}

It has been established that both 785 and $830 \mathrm{~nm}$ simple optical traps can be used to manipulate hyphal growth and branching $[7,35,36,39]$. Here we examined the effects of 532 and $1064 \mathrm{~nm}$ light, and included further experiments with $785 \mathrm{~nm}$ light for comparison.

The range of powers needed to cause a change in the pattern of hyphal growth varied largely with wavelength: for $1064 \mathrm{~nm}$ light $150-500 \mathrm{~mW}$, for $785 \mathrm{~nm} \mathrm{40-70} \mathrm{mW,} \mathrm{and} \mathrm{for}$ $532 \mathrm{~nm}$ light $1.9-14 \mathrm{~mW}$. For each range stated the minimum power was the lowest to cause an observable effect and the maximum was the highest to cause an effect without producing irreparable damage to the hyphae. The laser powers quoted are those incident on the back aperture of the microscope objective where the focal spot diameter is approaching the diffraction limit for each wavelength.

Examples of changed growth patterns for each wavelength are shown in figure 4 . The altered growth effect in each case is clearly associated with the proximity of the hyphal tip (and thus Spitzenkörper) to the optical trap. Once the hyphal tips had passed the trap, normal growth resumed back along its original growth direction unless growth was stopped because a hyphal tip was exposed to too high a laser power. In all experiments it was found to be important to judiciously use the minimum laser power necessary to cause a change in the hyphal growth pattern otherwise growth would commonly be stopped.

With all the wavelengths studied, the alteration is short lived, both in time and distance. The usefulness of the techniques described here would be greatly increased if the

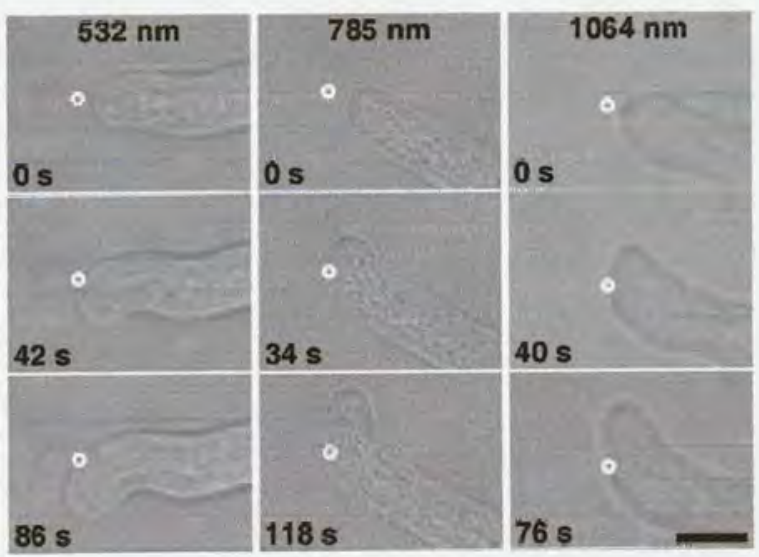

Figure 4. Examples of redirecting hyphal growth using 532, 785 and $1064 \mathrm{~nm}$ single Gaussian beam traps. The power required to cause the effect shown is between $1.9-14 \mathrm{~mW}$ at $532 \mathrm{~nm}, 40-70 \mathrm{~mW}$ at $785 \mathrm{~nm}$ and $150-500 \mathrm{~mW}$ at $1064 \mathrm{~nm}$. The white circle indicates the position of the optical trap. Bar $=10 \mu \mathrm{m}$.

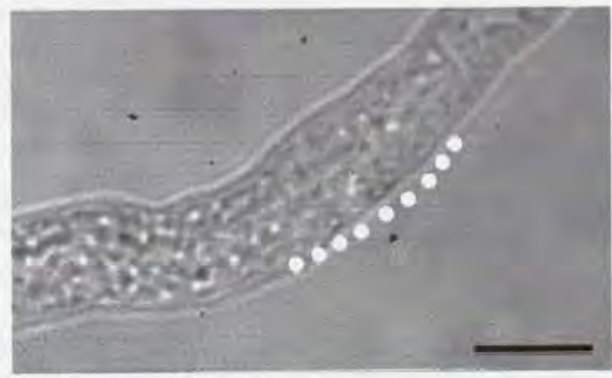

Figure 5. Continuous hyphal redirection using nine $532 \mathrm{~nm}$ Gaussian traps produced with HOTs and objective A. The hyphal tip grew from bottom left to top right. White dots represent the position of each focused trap. The laser power per tweezer spot was $\sim 2-3 \mathrm{~mW}$. Bar $=10 \mu \mathrm{m}$.

ability to alter growth could occur passively over relatively extended distances.

\subsection{Multiple Gaussian beams}

The holographic techniques described allow arbitrary patterns of multiple tweezers to be created for attempts to alter growth over an extended range. Figure 5 shows the result of allowing a hyphal tip to grow into a curve made from nine individual tweezers, produced using the holographic techniques described. 


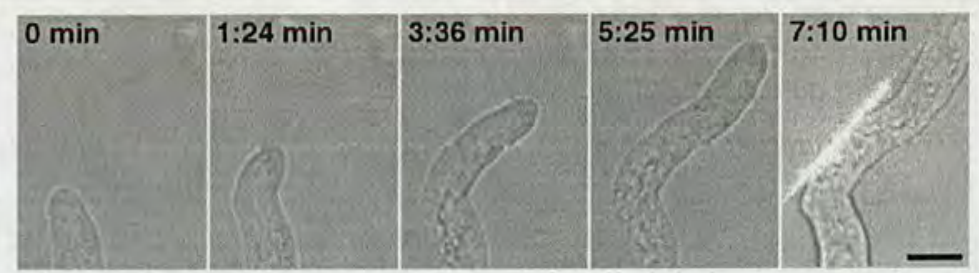

Figure 6. Extended redirection of hyphal growth using a single continuous line of $532 \mathrm{~nm}$ light produced with objective A and introduced at $57^{\circ}$ to the original axis of hyphal growth at time $0 \mathrm{~min}$. The final image in the sequence was captured after the laser filter was removed from its location in front of the camera, allowing visualization of the laser pattern and position. The total laser power spread over the whole pattern was $8.1 \pm 0.1 \mathrm{~mW}$. Bar $=10 \mu \mathrm{m}$.

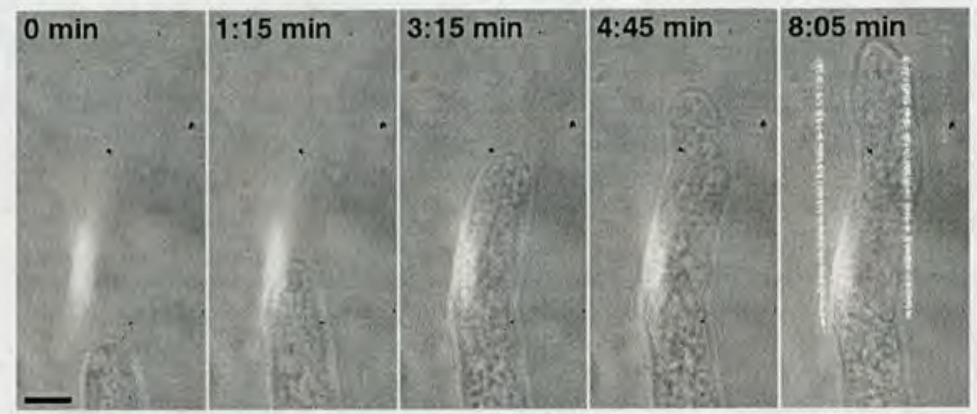

Figure 7. Guiding a hyphal tip through a $17 \mu \mathrm{m}$ wide channel bordered by $60 \mu \mathrm{m}$ long lines of light produced with objective B. The final image in the sequence was captured after the laser filter was removed to show the laser pattern and position. The white 'smudge' appearing in each image is an optical artefact. The total laser power spread over the whole pattern was $16.0 \pm 0.3 \mathrm{~mW}$. Bar $=10 \mu \mathrm{m}$.

The results were wildly inconsistent, rarely ending in a tip following the full pattern of multiple tweezers. These unsuccessful results generally occurred for one of two reasons. Firstly, the spacing of the tweezers often allowed hyphal tips to grow through the gaps between the tweezers without causing redirection. Secondly, if the hyphae grew through the tweezers their growth could cease due to overexposure to the laser beam.

To minimize these factors the light needs to be evenly distributed along the desired alteration pattern. As discussed, this is possible through the use of holography to create continuous light patterns.

\subsection{Continuous light patterns}

Generating single, continuous, $30 \mu \mathrm{m}$ long lines of light at an angle of $\geqslant 50^{\circ}$ to the direction of growth consistently caused hyphal redirection (figure 6). Inputting parallel laser lines in the software produced a 'channel' of light through which hyphal tips could be guided (figure 7). In instances where the hypha was wider than the light channel the width of the hypha became narrower as it entered the channel (figure 8 ). The first point of interaction between the hyphal tip and the light induced branch formation (figure 8; the last images shown in the two series of images in figures 6 and 9). Hyphal branching never occurred within a channel bordered by light or from a hypha growing up against a line of light.

It was simple to rapidly switch between pre-calculated kinoforms and hence optical fields in the focal plane. Figure 9 shows two sharp continuous redirections using this approach. Having redirected the hyphae at an angle of $45^{\circ}$ by time $4 \mathrm{~min}$ $44 \mathrm{~s}$, the kinoform was changed to produce a second line of light redirecting the hyphae by another $53^{\circ}$ by time $10 \mathrm{~min}$ $27 \mathrm{~s}$.

It was difficult to calibrate the laser power within the light patterns created. The holograms that produced lines of light parallel to the SLM axes have a higher diffraction efficiency over their diagonal counterparts. Thus, changing between diagonal and parallel lines of the same length also produced a change in intensity. We carefully selected the laser power input into the system by following a simple procedure. First, we made an estimate of the power required, and then we attempted to manipulate hyphal growth. If unsuccessful the power was either increased or decreased depending on whether the hyphae had been unaffected or overly affected, respectively. It was also at times necessary to search and find a new hyphal tip to experiment on had we overestimated the power needed and damaged the hyphal tip.

A pseudowall of light (figure 3 ), as described in section 2.3, improved the precision of growth redirection (figure 10). To produce the pseudowall of light the hologram used for figure 6 was axially stacked to produce nine axial planes of light, four each side of the normal focus, each separated by $0.5 \mu \mathrm{m}$. The hyphae consistently followed the edge of the pattern more closely with this optical setup than with those used in the previous experiments described above.

\section{Discussion}

We have shown that hyphal tip growth in $N$. crassa can be redirected using optical tweezers with three different wavelengths of light $(532,785$ and $1064 \mathrm{~nm})$, and the lower the 


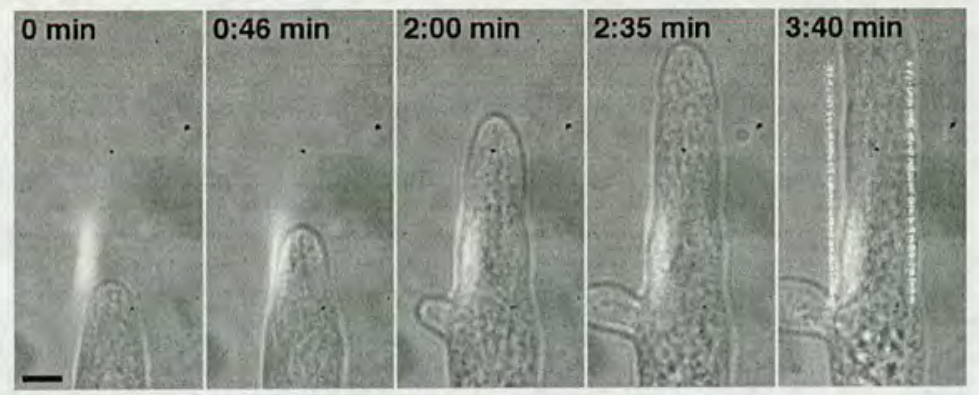

Figure 8. Hyphal constriction and branching caused by a relatively thinner channel (17 $\mu \mathrm{m}$ wide) bordered by $60 \mu \mathrm{m}$ long lines of light produced with objective B. The final image in the sequence was captured after the laser filter was removed to show laser pattern and position. The white 'smudge' appearing in each image is an optical artefact. The total laser power spread over the whole pattern was $9.0 \pm 0.2 \mathrm{~mW}$. $\mathrm{Bar}=10 \mu \mathrm{m}$.

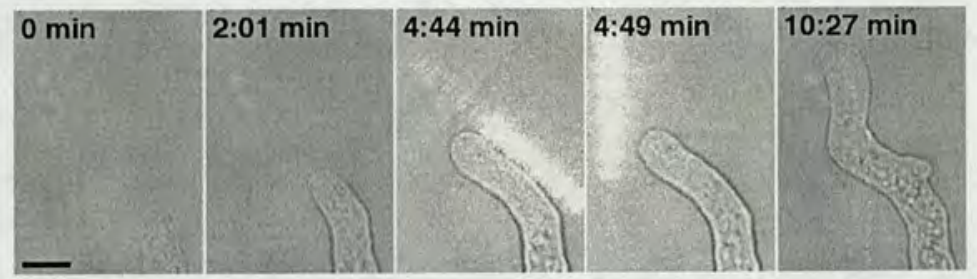

Figure 9. Multiple redirections of hyphal growth caused by switching between different kinoforms produced with objective A. The switch in light patterns took place between 4:44 and 4:49 $\mathrm{min}$ at which time the laser filter was removed to show laser pattern and position. The total laser power spread over each light pattern was $8.1 \pm 0.1 \mathrm{~mW}$. Bar $=10 \mu \mathrm{m}$.

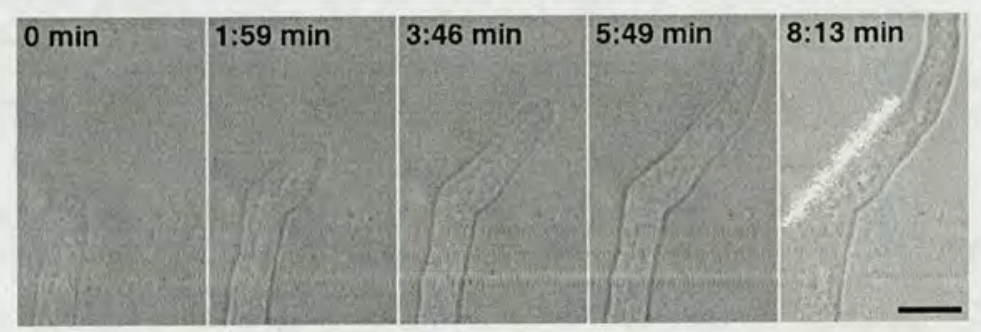

Figure 10. Extended hyphal growth redirection using a pseudo 3D wall of $532 \mathrm{~nm}$ light produced with objective $A$. The final image in the sequence was captured after the laser filter was removed to show the laser pattern and position. The total laser power spread over the whole pattern was $8.1 \pm 0.1 \mathrm{~mW}$. Bar $=10 \mu \mathrm{m}$.

wavelength used, the lower the laser power required to effect these changes.

As the reader may have noted we have not included an average power, but rather ranges at which hyphal growth alteration occurs for each wavelength. This is because the values are largely dependent on the size of the hyphal tips being studied and the patterns being produced. For a given run of experiments an effective power could quickly be found using trial and error and we aimed to minimize the level of irradiation whilst still eliciting a response in the pattern of hyphal growth.

When the range of laser intensities required at the focal plane of the tweezers was calculated, assuming a diffraction limited focus, it was found that lower wavelengths required lower intensities to effect a growth response, indicating a wavelength dependence. The cause of this is unknown.

Using an SLM designed for 400-700 nm operation, we can use digital holography to produce fixed patterns of light that can redirect or constrict hyphal growth over extended distances or initiate hyphal branching.
With all the optical methods employed to alter growth it was possible to use either too little or too much power. If too little power was used then hyphal tip growth was unaffected and the hyphae grew through the light. If too high a laser power was used then hyphal growth ceased, a response common in filamentous fungi exposed to stress (e.g. mechanical or osmotic). In the particular case of single optical tweezers, too high a power could cause hyphae to burst resulting in the cytoplasm leaking from the hyphal tip. A number of papers have assessed the damage caused to a variety of biological specimens by optical tweezers [61-63]. Potential sources for photodamage were cited as the generation of reactive oxygen species, two-photon absorption and transient local heating. It will be interesting to analyse the possible influence of these factors in future work.

It has previously been proposed that repulsion of the Spitzenkörper organelle complex by the optical trap is responsible for the variety of growth responses observed [7, 35, 36]. The work presented here provides further evidence to support 
this. It was only when the optical tweezer or light pattern was positioned at the hyphal apex that redirection of tip growth took place. Repeatedly switching the irradiation of the growing hyphal apex from one side to the other resulted in a repeated redirection of the growth axis giving rise to a zigzag pattern of filamentous growth $[30,36]$. Figure 4 shows that even though the gaps between multiple optical traps were much smaller than the width of the hyphal tip it was not smaller than the Spitzenkörper (figure 1(b)), which could therefore fit through the gaps without being influenced by the laser. Lines (figure 6) and 'pseudowalls' (figure 10) of light were shown to be much more effective at redirecting hyphal growth and when sufficient power was used the hyphal tips were unable to grow across the light barriers.

It is not possible to say whether the effects of light at the green, near-infrared and infrared wavelengths used are a physical phenomenon (e.g. optical repulsion of the Spitzenkörper [35], or aversion to localized heating), local intracellular generation of reactive oxygen species [61], or a photoreceptor-mediated negative phototropism [35]. Future work will test the latter hypothesis by repeating these experiments using strains in which the genes encoding different photoreceptors have been mutated [28], testing a greater range of wavelengths and attempting to image the behaviour of the Spitzenkörper during redirection.

We believe the growth alteration for both discrete and continuous patterns of light cannot be explained simply by the physics but must involve some unknown biological phenomenon.

The reason why hyphal branches were induced when hyphae became constricted by growing into narrow channels bordered by light is unclear. It is possible that the initial perturbation of hyphae by laser irradiation resulted in branch forming. Hyphal branch induction was often observed at the point of initial exposure to a laser trap [36] and branching has been initiated by using tweezers to apparently concentrate secretory vesicles within hyphae [7].

The axial extent of the two-dimensional continuous light patterns produced was very small compared to the actual vertical extent of the hyphal tips $(<18 \mu \mathrm{m})$. The alignment of the system and point of focus are thus highly critical. In order to increase the alignment tolerance, 'pseudowalls' of light were investigated (figure 10) and found to produce very consistent results in terms of redirecting hyphal growth. Also, the hyphae seemed less perturbed by the walls and followed the barriers much more closely than the single line of light (figure 6). Two possible reasons for the improvement in results obtained with the 3D pseudowalls of light are as follows. Firstly the same total power was used for both the pseudowalls and lines of light. As the pseudowall was spread between nine individual planes then at any one point the light was less intense, and thus less damaging to the cells. Secondly the fact the pseudowalls were extended axially means that given a misalignment in the system there was a higher chance of the hyphal tip and Spitzenkörper being coplanar with the light field. We foresee that one of the main advantages of holographic beam shaping is its ability to produce light patterns with axial extent, unlike, for example, AODs. To increase the tolerance still further, the possibility of creating true 3D walls of light [64] will be explored in future work.

\section{Conclusion}

Optical tweezers provide a useful tool to manipulate the pattern of growth in filamentous fungi. Using more advanced techniques offers the potential to create artificial networks of hyphae in three dimensions by growing these filamentous organisms through light mazes. Fungal hyphae within mycelial networks sense and respond to each other in complex ways which regulates the morphology of the colony. Being able to precisely manipulate the three-dimensional nature of these networks with light mazes may provide a useful experimental method to analyse cell-to-cell communication in these complex systems.

\section{Acknowledgments}

DRB and GDW would like to thank EPSRC for support. DM is a Royal Society University Research Fellow.

\section{References}

[1] Weibel D B, Garstecki P, Ryan D, Diluzio W R, Mayer M, Seto J E and Whitesides G M 2005 Proc. Natl Acad. Sci. 10211963

[2] Stevenson D J, Lake T K, Agate B, Garces-Chavez V, Dholakia K and Gunn-Moore F 2006 Opt. Express 149786

[3] Ashkin A, Dziedzic J M, Bjorkholm J E and Chu S 1986 Opt. Lett. 11288

[4] Ashkin A, Dziedzic J M and Yamane T 1987 Nature 330769

[5] Ashkin A and Dziedzic J M 1987 Science 2351517

[6] Leitz G, Schnepf E and Greulich K O 1995 Planta 197278

[7] Bracker C E, Murphy D J and Lopez-Franco R 1997 Proc. SPIE 298367

[8] Albrecht-Buehler G 1991 J. Cell Biol. 114493

[9] Ehrlicher A, Betz T, Stuhrmann B, Koch D, Milner V, Raizen M G and Kas J 2002 Proc. Natl Acad. Sci. 9916024

[10] Stuhrmann B, Gögler M, Betz T, Ehrlicher A, Koch D and Käs J 2005 Rev. Sci. Instrum. 76035105

[11] Berns M W, Aist J R, Wright W H and Liang H 1992 Exp. Cell Res. 198375

[12] Tolić-Nørrelykke I M, Sacconi L, Stringari C, Raabe I and Pavone F S 2005 Curr. Biol. 151212

[13] Leitz G, Lundberg C, Fällman E, Axner O and Sellstedt A 2003 FEMS Microbiol. Lett. 22497

[14] Liu et al 2004 J. Biotechnol. 109217

[15] Block S M, Blair D F and Berg H C 1989 Nature 338514

[16] Finer J T, Simmons R M and Spudich J A 1994 Nature 368113

[17] Greulich K O 2005 ChemPhysChem 62458

[18] Kuo S C and Sheetz M P 1993 Science 260232

[19] Cluzel P, Lebrun A, Heller C, Lavery R, Viovy J L, Chatenay D and Caron F 1996 Science 271792

[20] Wang M D, Yin H, Landick R, Gelles J and Block S M 1997 Biophys. J. 721335

[21] Greulich K O and Pilarczyk G 1998 Cell Mol. Biol. 44701

[22] Baumann C G, Bloomfield V A, Smith S B, Bustamante C, Wang M D and Block S M 2000 Biophys. J. 781965

[23] Greulich K O, Pilarczyk G, Hoffmann A, Horste G M Z, Schafer B, Uhl V and Monajembashi S 2000 J. Microsc. 198182

[24] Lang M J, Fordyce P M and Block S M 2003 J. Biol. 26

[25] Block S M 1992 Nature 360493

[26] Wang Y, Botvinick E L, Zhao Y, Berns M W, Usami S, Tsien R Y and Chien S 2005 Nature 4341040

[27] Kuo S C and Sheetz M P 1992 Trends Cell Biol. 2166

[28] Borkovich K A et al 2004 Microbiol. Mol. Biol. Rev. 681 
[29] Read N D 2007 Environmental sensing and the filamentous fungal lifestyle Fungi in their Environment ed G D Gadd, S C Watkinson and P S Dyer (Cambridge: Cambridge University Press) p 38

[30] Bartnicki-Garcia S 2002 Hyphal tip growth: outstanding questions Molecular Biology of Fungal Development ed H D Osiewacz (New York: Dekker) p 29

[31] Harris S D, Read N D, Roberson R W, Shaw B, Seiler S, Plamann M and Momany M 2005 Eukaryot. Cell 4225

[32] Davis R H 2000 Neurospora: Contributions of a Model Organism (Oxford: Oxford Univerity Press)

[33] Perkins D D and Davis R H 2000 Fungal Genet. Biol. 31153

[34] Galagan J E et al 2003 Nature 422859

[35] Wright G D, Arlt J, Poon W C K and Read N D 2007 Fungal Genet. Biol. 441

[36] Wright G D, Arlt J, Poon W C K and Read N D 2007 Mycoscience $\mathbf{4 8} 15$

[37] Buller A H R 1993 Researches on Fungi vol 5 (London: Longman)

[38] Hickey P C, Swift S R, Roca M G and Read N D 2005 Methods in Microbiology vol 34 (Amsterdam: Elsevier) p 63

[39] Wright G D, Arlt J, Poon W C K and Read N D 2005 Proc. SPIE $5930 \mathrm{~F} 1$

[40] Lopez-Franco R and Bracker C E 1996 Protoplasma 19590

[41] Mio C, Gong T, Terray A and Marr D W M 2000 Rev. Sci. Instrum. 712196

[42] Visscher K, Gross S P and Block S M 1996 IEEE J. Quantum Electron. 21066

[43] Dufresne E R and Grier D G 1998 Rev. Sci. Instrum. 691974

[44] Liesener J, Reicherter M, Haist T and Tiziani H J 2000 Opt. Commun. 18577

[45] Curtis J E, Koss B A and Grier D G 2002 Opt. Commun. 207169

[46] Sinclair G, Jordan P, Courtial J, Padgett M, Cooper J and Laczik Z J 2004 Opt. Express 125475
[47] Burnham D R and McGloin D 2006 Opt. Express 144175

[48] Melville H, Milne G F, Spalding G C, Sibbett W, Dholakia K and McGloin D 2003 Opt. Express 113562

[49] Arneborg N, Siegumfeldt H, Andersen G H, Nissen P, Daria V R, Rodrigo P J and Gluckstad J 2005 FEMS Microbiol. Lett. 245155

[50] Daria V R, Rodrigo P J and Gluckstad J 2004 Biosens. Bioelectron. 191439

[51] Lafong A, Hossack W J, Arlt J, Nowakowski T J and Read N D 2006 Opt. Express 143065

[52] Kohler C, Schwab X and Osten W 2006 Appl. Opt. 45960

[53] Gerchberg B W and Saxton W O 1972 Optik 35237

[54] Haist T, Schonleber M and Tiziani H J 1997 Opt. Commun. 140299

[55] McGloin D, Spalding G C, Melville H, Sibbett W and Dholakia K 2003 Opt. Express 11158

[56] Garces-Chavez V, McGloin D, Summers M D, Fernandez-Nieves A, Spalding G C, Cristobal G and Dholakia K 2004 J. Opt. A: Pure Appl. Opt. 6 S235

[57] Leach J et al 2006 Appl. Opt. 45897

[58] Soifer V, Kotlyar V and Dokolovich L 1997 Iterative Methods for Diffractive Optical Elements (London: Taylor and Francis) p 15

[59] Wulff K D, Cole D G, Clark R L, DiLeonardo R, Leach J, Cooper J, Gibson G and Padgett M J 2006 Opt. Express 144169

[60] Love G D 1997 Appl. Opt. 361517

[61] Neuman K C, Chadd E H, Liou G F, Bergman K and Block S M 1999 Biophys. J. 772856

[62] Liang H, Vu K T, Krishnan P, Trang T C, Shin D, Kimel S and Berns M W 1996 Biophys. J. 701529

[63] Sacconi L, ToliT-Norrelykke I M, Stringari C, Antolini R and Pavone F S 2005 Appl. Opt. 442001

[64] Whyte G and Courtial J 2005 Appl. Opt. 7117 